\title{
THE TAURUS SPITZER SURVEY: NEW CANDIDATE TAURUS MEMBERS SELECTED USING SENSITIVE MID-INFRARED PHOTOMETRY
}

L. M. Rebull ${ }^{1}$, D. L. Padgett ${ }^{1}$, C.-E. McCabe ${ }^{1}$, L. A. Hillenbrand ${ }^{2}$, K. R. StapelfeldT $^{3}$, A. Noriega-Crespo ${ }^{1}$, S. J. Carey ${ }^{1}$, T. Brooke ${ }^{1}$, T. Huard ${ }^{4}$, S. Terebey ${ }^{5}$, M. Audard ${ }^{6,7}$, J.-L. Monin ${ }^{8}$, M. Fukagawa ${ }^{9}$, M. GüdeL ${ }^{10}$, G. R. KnapP ${ }^{11}$,

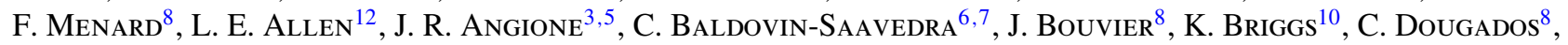
N. J. Evans ${ }^{13}$, N. Flagey ${ }^{1}$, S. Guieu ${ }^{1}$, N. Grosso ${ }^{14}$, A. M. Glauser ${ }^{10,15}$, P. Harvey ${ }^{13}$, D. Hines ${ }^{16}$, W. B. Latter ${ }^{17}$, S. L. SKINNER ${ }^{18}$, S. Strom ${ }^{12}$, J. TromP ${ }^{5}$, AND S. WOLF ${ }^{19}$

${ }^{1}$ Spitzer Science Center/Caltech, M/S 220-6, 1200 East California Boulevard, Pasadena, CA 91125, USA; luisa.rebull@jpl.nasa.gov

2 Department of Astronomy, California Institute of Technology, Pasadena, CA, USA

3 Jet Propulsion Laboratory, California Institute of Technology, Pasadena, CA, USA

${ }^{4}$ University of Maryland, College Park, MD, USA

${ }^{5}$ California State University, Los Angeles, CA, USA

${ }^{6}$ ISDC Data Center for Astrophysics, University of Geneva, Ch. d'Ecogia 16, CH-1290 Versoix, Switzerland

${ }^{7}$ Observatoire de Genève, University of Geneva, Ch. des Maillettes 51, 1290 Versoix, Switzerland

${ }^{8}$ Laboratoire d'Astrophysique de Grenoble, Université de Grenoble-CNRS, UMR 5571, Grenoble, France

${ }^{9}$ Department of Earth and Space Science, Graduate School of Science, Osaka University, 1-1 Machikaneyama, Toyonaka, Osaka 560-0043, Japan

${ }^{10}$ ETH Zurich, Institute of Astronomy, 8093 Zurich, Switzerland

${ }^{11}$ Princeton University, Princeton, NJ, USA

${ }^{12}$ NOAO, Tucson, AZ, USA

13 University of Texas, Austin, TX, USA

${ }^{14}$ Observatoire Astronomique de Strasbourg, Université de Strasbourg, CNRS, INSU, 11 rue de l'Université, 67000 Strasbourg, France

${ }^{15}$ UK Astronomy Technology Centre, Royal Observatory, Edinburgh EH9 3HJ, UK

${ }^{16}$ Space Science Institute, Boulder, CO, USA

17 NASA Herschel Science Center, IPAC, Pasadena, CA 91125, USA

${ }^{18}$ CASA, University of Colorado, Boulder, CO 80309-0389, USA

${ }^{19}$ University of Kiel, Institute of Theoretical Physics and Astrophysics, Leibnizstrasse 15, 24098 Kiel, Germany

Received 2009 July 29; accepted 2009 December 10; published 2010 January 21

\begin{abstract}
We report on the properties of pre-main-sequence objects in the Taurus molecular clouds as observed in seven mid- and far-infrared bands with the Spitzer Space Telescope. There are 215 previously identified members of the Taurus star-forming region in our $\sim 44 \mathrm{deg}^{2}$ map; these members exhibit a range of Spitzer colors that we take to define young stars still surrounded by circumstellar dust (noting that $\sim 20 \%$ of the bona fide Taurus members exhibit no detectable dust excesses). We looked for new objects in the survey field with similar Spitzer properties, aided by extensive optical, X-ray, and ultraviolet imaging, and found 148 new candidate members of Taurus. We have obtained follow-up spectroscopy for about half the candidate sample, thus far confirming 34 new members, three probable new members, and 10 possible new members, an increase of $15 \%-20 \%$ in Taurus members. Of the objects for which we have spectroscopy, seven are now confirmed extragalactic objects, and one is a background Be star. The remaining 93 candidate objects await additional analysis and/or data to be confirmed or rejected as Taurus members. Most of the new members are Class II M stars and are located along the same cloud filaments as the previously identified Taurus members. Among non-members with Spitzer colors similar to young, dusty stars are evolved Be stars, planetary nebulae, carbon stars, galaxies, and active galactic nuclei.
\end{abstract}

Key words: circumstellar matter - infrared: stars - stars: formation - stars: pre-main sequence

Online-only material: figure sets, machine-readable tables

\section{INTRODUCTION}

A complete inventory of all the coeval stars in a young stellar association, cluster, or group (hereafter "association") enables studies of the initial mass function (IMF), disk fraction, and stellar rotational properties, among other pursuits. Information from associations with a range of ages enables understanding of the overall formation and evolution of young stars, including the change with time of disk fraction and stellar rotation rate. However, identifying all of the member stars of a given young association can be quite difficult. (By "member," we mean objects that are clearly young, close to the same age, and often still associated with their natal cloud.) Finding all such members requires that one employ multiple observational techniques. These methods include but are not limited to X-ray surveys (e.g., Alcalá et al. 1996; Wolk et al. 2006), H $\alpha$ surveys (e.g., Ogura et al. 2002), variability surveys (e.g., Carpenter et al. 2001; Rebull 2001), ultraviolet (UV) surveys (e.g., Rebull et al. 2000), and infrared (IR) surveys (e.g., Jørgensen et al. 2006; Rebull et al. 2007). At each wavelength, we can use the fact that stellar youth implies more flux at a given radiometric band (X-rays, $\mathrm{H} \alpha$, UV, IR), or more flux variability, than older stars of comparable mass, allowing separation of association members from field contaminants. When we combine the information from surveys in multiple wavelengths, we must remember that the influence of extinction due to circumstellar matter and/or the molecular cloud is vastly different at different wavelengths; extinction affects UV and optical wavelengths much more strongly than IR. For young associations, many, but not all, legitimate members are identifiable using just one or a few survey methods.

There are advantages and disadvantages to studying nearby young associations. Identification of young members in an 
association is made easier if the objects are located at smaller heliocentric distances and therefore brighter on the whole. This is especially the case for low-mass members with very low luminosities; a complete census of these stars and brown dwarfs can be made only for very nearby star-forming regions. On the other hand, nearby star-forming molecular clouds cover large areas of sky and therefore require large investments of observing time. At just $137 \mathrm{pc}$ (with a depth of $\sim 20 \mathrm{pc}$; Torres et al. 2007, 2009), the Taurus star-forming region is one of the closest large cloud complexes with hundreds of low-mass stars, ongoing star formation, and objects ranging in age up to $\sim 5$ Myr. Studies of Taurus objects have significantly influenced our basic understanding of the star formation process for decades (e.g., Herbig \& Kameswara Rao 1972; Kenyon et al. 2008). However, the Taurus Molecular Cloud (TMC) is close enough that it subtends more than $100 \mathrm{deg}^{2}$ of sky; surveying all or even most of it is difficult within typical telescope time allocations.

IR surveys led to the discovery that some stars have IR excesses, interpreted as circumstellar matter (e.g., Aumann et al. 1984; Beichman et al. 1986). Most if not all low-mass stars form with circumstellar accretion disks, resulting in IR excesses for as long as the dusty circumstellar material survives (e.g., Hernandez et al. 2008). By using IR to survey a starforming region, the stars with IR excesses are relatively easily distinguished from stars without such excesses. The Spitzer Space Telescope (Werner et al. 2004) provides an excellent platform for surveying star-forming regions in the mid-IR and far-IR, enabling stars with IR excesses to be identified; the member stars which do not have IR excesses must be recovered using different techniques such as the ones listed above. Because Spitzer relatively efficiently maps large regions of sky, it is a particularly useful tool for surveying large star-forming regions.

We have conducted a large multiwavelength imaging and spectroscopic survey of the TMC in order to test if our inventory of Taurus members with IR excesses is, in fact, complete. The Spitzer imaging component is referred to as the Taurus Spitzer Survey, and is described by Padgett et al. (2008a, hereafter P08) and Padgett et al. (2010, in preparation, hereafter P10). It covers $\sim 44 \mathrm{deg}^{2}$ from 3.6 to $160 \mu \mathrm{m}$ and is a Spitzer Legacy Project, so enhanced data products have been delivered back to the Spitzer Science Center (SSC), including the catalogs on which this paper is based. In addition to the Spitzer component, there are four other major components to our Taurus survey. $X M M-N e w t o n$ was used by the XMM-Newton Extended Survey of the TMC (XEST) program (e.g., Güdel et al. 2007b and references therein), which mapped $\sim 5 \mathrm{deg}^{2}$, most of which was also mapped by the Spitzer observations; the XEST data include X-ray imaging but also include UV data from the XMM-Newton Optical Monitor (OM; Audard et al. 2007). XEST was deliberately pointed toward aggregates of previously identified Taurus members. In the optical, the Canada-FranceHawaii Telescope (CFHT) survey (J.-L. Monin et al. 2010, in preparation; Güdel et al. 2007b) mapped $\sim 28 \mathrm{deg}^{2}$ (all of which are encompassed by the Spitzer area), and the Sloan Digital Sky Survey (SDSS; Finkbeiner et al. 2004; Padmanabhan et al. 2008) mapped $\sim 48 \mathrm{deg}^{2}$ in two perpendicular strips, about half of which overlaps the Spitzer area. Finally, the Five College Radio Astronomy Observatory (FCRAO) millimeter wavelength survey (Goldsmith et al. 2008) mapped $\sim 100 \mathrm{deg}^{2}$ in the $\mathrm{CO}(1-0)$ line, covering the Spitzer survey area entirely. The relative coverage of these surveys is shown in P10. Our extended collaboration has already begun to use this rich data set to search for new members of Taurus; Scelsi et al. (2007,
2008) identified new candidate members using the XEST data, and Guieu et al. $(2006,2007)$ identified new brown dwarf members using the CFHT data to study their disk properties using the Spitzer data. Ongoing investigations include searches for members via emission line spectra (G. Knapp et al. 2010, in preparation), Herbig-Haro (HH) objects (K. R. Stapelfeldt et al. 2010, in preparation), and transition disks (C.-E. McCabe et al. 2010 , in preparation).

In this paper, we select new candidate Taurus members with IR excesses using Spitzer and Two Micron All Sky Survey (2MASS; Skrutskie et al. 2006) data. We construct colormagnitude and color-color diagrams for point sources, then use the locations of previously identified young stars in these diagrams to select new candidate members with IR excesses. To discard obvious extragalactic sources, we examine the source morphology in all available bands of the multiwavelength Taurus survey. We construct spectral energy distributions (SEDs) from the photometry over all available bands, again discarding objects we believe to be galaxies. Follow-up spectroscopy has been obtained to assess whether or not our new candidate Taurus objects are likely Taurus members. We also present Spitzer flux densities for the 215 previously identified members found in the region covered by our Spitzer survey. Note that (a) the 215 previously identified objects include those members without IR excesses identified via other mechanisms; (b) the 215 previously identified objects are those covered by our Spitzer map-there are other legitimate Taurus members outside the region we observed, such as in L1551; (c) our new candidate member list is necessarily just those with IR excesses and exclusively within the regions covered by our Spitzer observations. Some objects are resolved in one or more of the Spitzer images, and extended source photometry may be a better representation of the complete flux from the object; many of the extended sources are discussed individually in other papers (e.g., Tobin et al. 2008; K. R. Stapelfeldt et al. 2010, in preparation).

The observations, data reduction, and ancillary data are described in Section 2. Section 3 describes our young stellar object (YSO) selection process which is based on the colors of previously identified Taurus members, also presented here. We describe 34 objects that we have, thus far, identified as new members of Taurus (plus 3 probable new members and 10 possible new members) in Section 4 and discuss the properties of the new objects in conjunction with (and comparison to) the previously identified members of Taurus. Finally, we summarize our main points in Section 5. The appendix contains SEDs and discussion of some specific objects.

\section{OBSERVATIONS, DATA REDUCTION, AND ANCILLARY DATA}

\subsection{Spitzer Data}

P08 and P10 present a comprehensive discussion of the Spitzer data acquisition, reduction, and bandmerging to the 2MASS data. In summary, we conducted the observations using Infrared Array Camera (IRAC; 3.6, 4.5, 5.8, and $8 \mu \mathrm{m}$; Fazio et al. 2004) and Multi-band Imaging Photometer (MIPS for Spitzer; 24, 70, and $160 \mu \mathrm{m}$; Rieke et al. 2004) in two epochs to enable removal of asteroids from the final point-source catalog. The observations were spread over three observing programs and three years, 2005-2007.

For IRAC, we used MOsaicking and Point source EXtractor (MOPEX; Makovoz \& Marleau 2005) to find the sources, and IDL to perform aperture photometry at those locations. With 
our IRAC observations, we sacrificed redundancy for spatial coverage, and obtained just two IRAC frames per position (total integration time of $25.2 \mathrm{~s}$ ), so instrumental artifacts are abundant. We discarded single-band (apparent) detections as likely artifacts. Objects that we measured to be brighter than the $0.6 \mathrm{~s}$ saturation limits $(630,650,4600$, and $2500 \mathrm{mJy}$ at the four IRAC channels) we took to be saturated, and these appear as lower limits in our catalog. The zero-points we used to convert between flux densities and magnitudes were as found in the IRAC Data Handbook on the SSC Web site: 280.9, 179.7, 115.0, and 64.13 Jy for IRAC's four channels, respectively.

For MIPS, we used MOPEX point-response function (PRF) fitting photometry at 24 and $70 \mu \mathrm{m}$. The total integration time at $24 \mu \mathrm{m}$ was $30 \mathrm{~s}$ per position; the MIPS scan legs were interleaved to provide complete coverage at $70 \mu \mathrm{m}$, and a total integration time of $15 \mathrm{~s}$ per position. We took objects brighter than $4.7 \mathrm{Jy}$ at $24 \mu \mathrm{m}$ and $6.5 \mathrm{Jy}$ at $70 \mu \mathrm{m}$ to be saturated (or at least nonlinear). These objects appear in our catalog as having lower flux density limits. Following the MIPS Data Handbook on the SSC Web site, the zero-points that convert between flux densities and magnitudes are 7.14 and 0.775 Jy for MIPS-24 and 70, respectively.

The observations at $160 \mu \mathrm{m}$ present special challenges. All of the things that can affect the other bands (such as saturation, extended emission, confusion with the cloud background and nearby objects-whether they be Taurus or background objects - and instrumental artifacts) also affect this band. Because the Taurus cloud emission and instrumental effects are both very strong, and because the resolution is the poorest of all the Spitzer bands, these items are of particular concern at this bandpass. Additionally, due to scattered data gaps in our $160 \mu \mathrm{m}$ map (see P08, P10), measurements (or limits) at $160 \mu \mathrm{m}$ are missing or compromised for some sources. MOPEX does not detect any point sources automatically at $160 \mu \mathrm{m}$, because all detected point-like objects appear to be slightly resolved. For $\sim 100$ objects that were apparent by eye in the $160 \mu \mathrm{m}$ image (or for which limits were of interest for this paper), we performed aperture photometry on the cleaned image which was smoothed by a 4 pixel median filter to minimize the influence of holes in the map and image artifacts. We used a $32^{\prime \prime}$ aperture, an annulus from $64^{\prime \prime}$ to $128^{\prime \prime}$, and an aperture correction of 1.97 (valid for temperatures between 500 and $2000 \mathrm{~K}^{20}$ ). Based on a comparison of the flux densities obtained from the filtered image and the unfiltered image, we took the flux density uncertainties to be $20 \%$ below $5 \mathrm{Jy}$ and $30 \%$ for higher flux densities. Some objects that fell in regions with too many missing flux density values (from saturation or gaps in the map) are not retrievable and thus do not have a measurement (or limits); in essence, they are off the edge of the map. Visual inspection of each $160 \mu \mathrm{m}$ source was used in order to determine whether the object was clearly detected as a single point source, confused with another nearby source, or contaminated by data dropouts and/or saturation issues which would cause the reported flux density to be a lower limit; these are indicated in the data tables below. The zero-point for MIPS-160 is $0.159 \mathrm{Jy}$, again from the MIPS Data Handbook on the SSC Web site.

We extracted $J H K_{\mathrm{s}}$ data from the 2MASS point-source catalog for our region, retaining flux densities only for those objects with high-quality 2MASS data flags. There were a handful of objects of interest for this paper for which we

\footnotetext{
20 The dust we see at $160 \mu \mathrm{m}$ is likely to be cooler than this, but the aperture correction changes only by $0.7 \%$ between this temperature range and $50 \mathrm{~K}$, well within the uncertainties.
}

made the following manual modifications, and therefore these modified values were used for the color-magnitude diagrams (CMDs) and SEDs below. Measurements for five objects did not meet the data flag criteria we imposed on the 2MASS catalog, but their as-reported flux densities were completely consistent with the rest of their SED, so we adopted their measurements as good detections; these objects are SST Tau 041542.7+290959, $041542.7+290959,042517.6+261750,043835.4+261041$, and $043354.7+261327$. Flux densities for one or more bands for the following previously identified Taurus members do not exist in the point-source catalog, and thus the flux densities used below were taken from the extended source catalog: SST Tau 042757.3+261918, 044112.6+254635, 043535.3+240819, and $043316.5+225320$.

We bandmerged all the available point sources between 2 and $70 \mu \mathrm{m}$ using position matching alone, with a wavelengthdependent maximum matching radius. Sources between 2 and $8 \mu \mathrm{m}$ were matched over a radius of $1^{\prime \prime}$, and at $24 \mu \mathrm{m}$, the matching radius to the rest of the catalog was $2^{\prime \prime}$. All matching radii are values empirically determined by inspection of histograms of nearest neighbors between bands, and spotchecking individual sources; histograms of positional offsets and additional discussion appears in P10. We pre-merged the 24 and $70 \mu \mathrm{m}$ catalogs before merging to the rest of the catalog using an empirically determined radius of $10^{\prime \prime}$. Because the spatial resolution of the $70 \mu \mathrm{m}$ images is so much worse than the $2 \mu \mathrm{m}$ images, often more than one NIR (or optical) source can be matched to the $70 \mu \mathrm{m}$ source; however, it is extremely likely that if we detect a source at $70 \mu \mathrm{m}$, it will also appear at $24 \mu \mathrm{m}$, so by implementing the pre-merge of 24 and $70 \mu \mathrm{m}$, we are preferentially matching the $70 \mu \mathrm{m}$ sources to a likely physical match.

We include here a brief aside on the accuracy of blind merging by position. In the generic case of surveys across wavelengths, relative astrometric accuracy and spatial resolution is paramount. In our case of a catalog primarily driven by Spitzer+2MASS sources, astrometric accuracy is not the dominant source of error, since each instrument is internally consistent and calibrated to the $2 \mathrm{MASS}$ coordinate system. For many star-forming regions studied with Spitzer, e.g., many of those observed by the Cores-to-Disks (c2d; Evans et al. 2003) and Gould's Belt (L. E. Allen et al. 2010, in preparation) projects, the source surface density is high enough that blind merging by position causes an unacceptable rate of false matches, and multiple short-wavelength sources should in reality be assigned to a single long-wavelength source. However, the source surface density in Taurus is low enough $(\sim 4$ sources per square arcminute, compared to $\sim 20$ in some star-forming regions) that source confusion in general is not as much of a concern. On the other hand, in Taurus, many objects are known to be close binaries, and we do not apportion the flux density we observe between the two objects; for close binaries, we have treated the object as single here. In any case, in three of the objects investigated in detail for this paper $(<1 \%$ of the objects investigated, $<0.5 \%$ of the total number of $70 \mu \mathrm{m}$ sources in the entire catalog), we determined by individual inspection that the 24/70 flux densities were incorrectly matched to a nearby faint short-wavelength source, rather than the correct, bright, slightly farther away, short-wavelength source. In these cases, we manually tweaked the flux assignment; the position change is well within the expected uncertainties between the long- and shortwavelength catalogs. We are confident that for the large majority of the sources in our entire catalog, our blind merging by 
position works, but for any particular source not discussed here, the images and the flux assignment in our delivered catalogs should be carefully scrutinized.

In creating our Spitzer-centric catalog, we dropped any object without a Spitzer detection (e.g., sources off the edges of our maps) before proceeding and after each additional merging step below (e.g., when combining the SDSS catalog and the Spitzer catalog, we did not retain SDSS-only sources from off the edges of our map or whose SED falls so rapidly that they are too faint for our shallow Spitzer survey). There are nearly 700,000 sources in the catalog with Spitzer flux densities in at least one band. This does not include the asteroids, which will be discussed by D. Hines et al. (2010, in preparation). It also does not include sources that are substantially extended (except at $160 \mu \mathrm{m}$, see below); many of those will be covered by K. R. Stapelfeldt et al. (2010, in preparation). The vast majority of the $\sim 700,000$ sources are well behind the TMC. Sources which are members are generally expected to be bright in the shorter bands and detected in multiple bands.

P10 discusses the survey sensitivity in detail. In summary, the 3.6 and $4.5 \mu \mathrm{m}$ sensitivities are quite comparable, and $98 \%$ of the objects detected in $3.6 \mu \mathrm{m}$ are also detected at $4.5 \mu \mathrm{m}$. The 5.8 and $8 \mu \mathrm{m}$ channels are much less sensitive; only $22 \%$ of the objects detected at $3.6 \mu \mathrm{m}$ are also detected at $5.8 \mu \mathrm{m}$, and just $17 \%$ of the objects detected at $3.6 \mu \mathrm{m}$ are also detected at $8 \mu \mathrm{m}$ (where nebulosity can also be a factor in pointsource identification and extraction). Our 24, 70, and $160 \mu \mathrm{m}$ sensitivity is a strong function of position in the image because of nebular and high Zodiacal dust emission contributions to the background. In part because of the varying background but also because of the effective sensitivity of the instrument to photospheres at the distance of Taurus given our exposure times, just $1.4 \%$ of the objects detected at $3.6 \mu \mathrm{m}$ are also detected at $24 \mu \mathrm{m}$, and just $0.15 \%$ are detected at $70 \mu \mathrm{m}$. The faintest independent $24 \mu \mathrm{m}$ detection is $\sim 10$ th mag ( $\sim \mathrm{M} 2$ spectral type photosphere at Taurus age and distance), and the histogram of detections turns over at $\sim 9.3 \mathrm{mag}$ (e.g., there is a steep fall-off between [24] $\sim 9.3$ and 10, where the bracket notation denotes the magnitude at that band). Similarly, at $70 \mu \mathrm{m}$, the faintest object is [70] 3.5, and a more typical value is 2.5-2.7 (note that $70 \mu \mathrm{m}$ is much more strongly affected by nebular emission and instrumental effects than $24 \mu \mathrm{m}$ ). Only $\mathrm{O}$ or B photospheres would be that bright at the distance of Taurus, so we can only detect legitimate Taurus objects at 70 (or 160) $\mu \mathrm{m}$ which have substantial IR excesses.

A full completeness analysis is given by P10, but as another independent way of assessing our completeness, we examined by hand each of the images at each of the Spitzer bands for each of the previously identified Taurus members and new candidate Taurus members discussed here (see Sections 3.1.1 and 3.1). If the object could be seen in the image but a flux density was not initially reported at that band, we made a manual assessment of the flux density or upper/lower limit, as appropriate. For IRAC, $\sim 1 \%-2 \%$ (depending on the band) of the previously identified Taurus members and $\sim 4 \%-11 \%$ of the new candidate members were missing photometry and were filled in manually (having lower signal to noise). The most common band in which flux densities were erroneously missing (e.g., not in the automatically generated catalog but visible in the images) was $5.8 \mu \mathrm{m}$, which is not particularly surprising, as this is the least sensitive of the IRAC bands. For MIPS, 9\% of the previously identified Taurus members were missing photometry in either 24 or $70 \mu \mathrm{m}$, and $\sim 6 \%$ of the new candidate objects were missing photometry at 24 or $70 \mu \mathrm{m}$. The lower fraction of missing photometry in the new candidate objects as compared to the previously identified objects is a reflection of the fact that our selection mechanism is somewhat biased toward objects with MIPS detections; see Section 4.1 below.

Many sources detected at shorter wavelengths are undetected at longer wavelengths, and it is important for our science analysis to obtain upper limits for our sources in the Spitzer photometric bands. For the list of coordinates of previously identified and new candidate Taurus members, at $24 \mu \mathrm{m}$, we used MOPEX to look for a source whose photometry could be obtained via PRF fitting at that location. If an object was detected, we took a weighted average flux density based on all detections (e.g., between epochs and tiles). If the final signal-tonoise ratio $(\mathrm{S} / \mathrm{N})$ was $<3$, we took the error to be the $1 \sigma$ upper limit, and multiplied by 3 to get the $3 \sigma$ limits found in the data tables below and SEDs in Appendix A. If the PRF fitting failed, we performed aperture photometry at the location of the object (at each tile and epoch available), and took a weighted average of all the resultant positive aperture flux densities. If that average had an $\mathrm{S} / \mathrm{N}>3$, we took that weighted average to be a detection. For all objects that had $\mathrm{S} / \mathrm{N}<3$, we took the upper limit to be the $1 \sigma$ limit (and multiplied by 3 to get the $3 \sigma$ limits found in the data tables and SEDs below). If all the measured aperture flux densities were negative, we took the $1 \sigma$ limit to be the straight average of the errors (and multiplied by 3 to get the $3 \sigma$ limits found in the tables and SEDs below). Finally, still at $24 \mu \mathrm{m}$, the objects that cannot be resolved from a companion are reported as simply unknown, where the presence of a companion is known from shorter-wavelength higher-spatial-resolution observations. At $70 \mu \mathrm{m}$, some objects are unresolved from a nearby object and are impossible to estimate (again, where the presence of a companion is known from shorter-wavelength higherspatial-resolution observations). Upper limits at $70 \mu \mathrm{m}$ for all remaining undetected sources were obtained by performing aperture photometry at the expected source location, using a $35^{\prime \prime}$ aperture and a multiplicative aperture correction of 1.22 , as discussed in the MIPS Data Handbook, available at the SSC Web site. These $1 \sigma$ errors were multiplied by 3 to get the $3 \sigma$ errors shown in the tables and figures here. Upper limits at $160 \mu \mathrm{m}$ were individually assessed using aperture photometry on the uncertainty image using the same parameters as for detected sources above (32" aperture, an annulus from $64^{\prime \prime}$ to $128^{\prime \prime}$, and an aperture correction of 1.97) to obtain $1 \sigma$ errors, and then multiplied by 3 to get the $3 \sigma$ errors shown in the tables and figures here.

\subsection{Complementary Survey Photometric Data}

In Section 2.1 above, we discussed merging the 2MASS and Spitzer data. We also need to match our catalog to the other Taurus surveys, listed in Section 1 above. Again, we match by position, with a radial offset tolerance customized empirically to each band or catalog.

The extracted CFHT point sources (see J.-L. Monin et al. (2010, in preparation) or Guieu et al. (2006) for more details on the extraction process; also see Monin et al. (2007) or Guieu (2008)) are merged first to 2MASS. Sources that are CFHT-only are then dropped in order to remove objects that are very statistically likely to be instrumental artifacts. The CFHT sources are then merged to the Spitzer catalog. The CFHT I bandpass is converted to Cousins $I$ via the following equation:

$$
I_{C}=I_{\mathrm{CFHT}}-0.531 \times\left(I_{\mathrm{CFHT}}-z_{\mathrm{CFHT}}\right)-0.278
$$


and then converted to flux densities using the Cousins $I$ zeropoint from Bessell (1979), $2.55 \times 10^{-23} \mathrm{~W} \mathrm{~m}^{-2} \mathrm{~Hz}^{-1}$. For these points, we use the $I_{C}$ effective wavelength of $0.79 \mu \mathrm{m}$. About 138,000 of the point sources in our catalog have CFHT $I_{C}$ magnitudes (20\% of the entire catalog).

The SDSS photometry arrives from the SDSS pipeline with photometric measurements in ugriz in flux density units of nanomaggies ${ }^{21}$ which can be converted to the same flux density units as the rest of the catalog data. We retained only those flux density measurements with good quality flags, and we merged the source lists for each of the SDSS tiles to each other by position to remove duplicates before merging to the master Spitzer catalog. The effective wavelengths are 3590, 4810, 6230, 7640 , and $9060 \AA$ for ugriz, respectively. We also made note of whether the object appearing in the SDSS images was flagged by the pipeline as extended or not. There are SDSS $z$-band flux densities for about 300,000 objects in our catalog ( $45 \%$ of the entire catalog). There are 6400 spectra available from SDSS, about 3400 of which overlap the Spitzer survey region; for each object, we matched by position to our Spitzer catalog, and accepted the spectral classification produced by the SDSS pipeline as a spectral classification of the object, unless another spectral classification was available in the literature (see below).

There are $\sim 1000$ objects in our catalog $(0.1 \%$ of the entire catalog) with X-ray measurements from the XEST survey (Güdel et al. 2007b). The XEST catalog includes flux densities from the XMM-Newton OM. The OM has a field of view comparable to, but not exactly identical to, the main X-ray field of view; see Audard et al. (2007) for more discussion of the XEST-OM sample. These data are in one of the three UV bandpasses ( $U$, UVW1, or UVW2). To convert these values to flux densities, we used the following equation, found in the XMM-Newton OM calibration document (Chen et al. 2004):

$$
F_{v}=10^{(0.4(Z-m))} \times f \times \lambda^{2} / c \times 10^{23},
$$

where $m$ is the reported magnitude (and $F_{v}$ the flux density) for a given object, $Z=18.259,17.204$, and 14.837 , and $f=$ $1.94 \times 10^{-16}, 4.76 \times 10^{-16}$, and $5.71 \times 10^{-15} \mathrm{erg} \mathrm{cm}^{-2} \mathrm{~s}^{-1} \AA^{-1}$ counts $^{-1} \mathrm{~s}$ for $U$, UVW1, and UVW2, respectively. In the equation, $\lambda$ is in units of $\AA$, and $c$ is $3 \times 10^{18} \AA \mathrm{s}^{-1}$. The effective wavelengths are $0.344,0.291$, and $0.212 \mu \mathrm{m}$ for $U$, UVW1, and UVW2, respectively. There are $\sim 1600$ objects with $X M M$ Newton OM flux densities in our catalog $(0.2 \%$ of the entire catalog).

We note that many of the X-ray detected XEST sources are likely background galaxies (see Güdel et al. 2007b) and that XEST included regions not covered by our map, such as L1551.

The XEST team assembled a catalog of supporting data from the literature, such as optical photometric measurements, for all of the previously identified Taurus members (see Section 3.1.1 below); we have included these photometric points in our database, converting Johnson magnitudes to flux densities using zero-points available in the literature (e.g., Cox 2000 and references therein).

The SEDs presented in this paper use all of these supporting data where available (except for the X-ray fluxes), and are presented as $\lambda F_{\lambda}$ in cgs units $\left(\mathrm{erg} \mathrm{s}^{-1} \mathrm{~cm}^{-2}\right.$ ), against $\lambda$ in $\mu \mathrm{m}$.

\footnotetext{
21 In SDSS, a "maggy" is the ratio of the flux density of the object to a standard flux density. The Sloan magnitudes are AB magnitudes, as opposed to Vega magnitudes. In the AB system, a flat spectrum object with $3631 \mathrm{Jy}$ at each band should have every magnitude equal to zero, and all maggies equal to one. Flux densities returned by the Sloan pipeline are nanomaggies, and can be converted to $\mu \mathrm{Jy}$. For more discussion, see Padmanabhan et al. (2008).
}

\subsection{Spectroscopy}

We obtained follow-up spectroscopy for $\sim 75 \%$ of the candidate YSOs discussed in this paper. Some previously identified Taurus members missing spectral types in our database (as discussed in Section 3.1.1 below) were also observed. Data were obtained over six runs between 2007 and 2009 at Keck and the Palomar 200". Most of the $\gtrsim 200$ spectra are low-resolution optical, obtained with the Double Spectrograph at Palomar (2008 November 30-December 3) or LRIS at Keck (2007 February). Many spectra were taken in the IR with Triplespec at Palomar (2008 November 21-24 and 2008 December 21) or NIRSPEC at Keck (2007 December and 2008 February). The IR spectra will be discussed in a forthcoming paper; the optical spectra are discussed here.

The optical spectra from Palomar are taken in two segments, blue and red; the blue covered $\sim 3710-5660 \AA$ at $\sim 2 \AA$ pixel $^{-1}$ and the red, $\sim 6230-8720 \AA$ at $\sim 2.5 \AA$ pixel $^{-1}$. Instrument settings were a 316 lines $\mathrm{mm}^{-1}$ grating blazed at $7500 \AA$ and used at grating angle 24.75 for the red side, and a 300 lines $\mathrm{mm}^{-1}$ grating blazed at $3900 \AA$ and used at grating angle 23.12 for the blue side. LRIS at Keck is also a double-barreled spectrograph which we used with a 400 lines $\mathrm{mm}^{-1}$ grism blazed at $3400 \AA$ in the blue and a 400 lines $\mathrm{mm}^{-1}$ grating blazed at $8500 \AA$ and positioned at grating angle 23.49 in the red. We obtained continuous wavelength coverage from the blue atmospheric cutoff to $\sim 9400 \AA$ at $1.86 \AA \mathrm{pixel}^{-1}$. The Double Spectrograph and LRIS data were both reduced using the Image Reduction and Analysis Facility (IRAF) ${ }^{22}$ ccdred and onedspec packages. Images were trimmed, bias-subtracted, and flattened prior to spectral extraction with the IRAF task apall. Wavelength calibration was performed using an Fe-Th-Ar lamp in the blue and $\mathrm{Th}-\mathrm{Ar}$ lamp in the red. The wavelength solution was applied using the IRAF task dispcor. The white dwarf Feige 34 was observed each night, providing an approximate flux calibration reference for all the scientific targets.

Spectral classification was obtained via visual examination of each spectrum and comparison to a standard grid composed of $>60$ stars ranging in types from B8 to M9. Four authors performed the classification independently to achieve an estimated accuracy of roughly a subclass.

The red spectra included $\mathrm{H} \alpha$. For each spectrum, we used IRAF to measure an equivalent width for $\mathrm{H} \alpha$, following the usual convention where negative values indicate emission (see Section 4.1). We also noted if the Ca IR triplet was in emission at the time of observation; this is indicated in the data tables below.

The spectroscopic data are sufficient to rule out redshifted galaxies, to classify stars, and to find stars with $\mathrm{H} \alpha$ in emission. However, our data are of insufficient resolution to, e.g., detect the presence of lithium, or determine surface gravities for most types. Section 4.1 discusses an analysis similar to that presented by Slesnick et al. (2008) which uses the TiO $8465 \AA$ index and $\mathrm{Na} 8190 \AA$ A index to determine an estimate of the surface gravity of the star. Additional follow-up data will be required to assess membership for stars with no $\mathrm{H} \alpha$ in emission and a small IR excess at Spitzer bands, and/or types earlier than M1 where the gravity analysis is not applicable.

\footnotetext{
22 IRAF is distributed by the National Optical Astronomy Observatories, which are operated by the Association of Universities for Research in Astronomy, Inc., under cooperative agreement with the National Science Foundation.
} 


\section{YSO CANDIDATE SELECTION}

\subsection{Overview of YSO Selection Process}

First, we establish two comparison samples from the literature, and then we discuss the process by which we selected new candidate YSOs in Taurus.

\subsubsection{Template Sample of Previously Identified Taurus Members}

We first informed our search for new Taurus members by determining the regions of color space occupied by previously identified Taurus members. By "Taurus member," we mean an object that is confirmed via multiple mechanisms to be young and associated with the TMC and the other Taurus members, e.g., sharing communal properties such as stellar activity. By "previously identified," we mean identified as a member by other authors in the literature using data sets other than the Taurus Spitzer Legacy Survey. However, in order to appear in our catalog, the object must be within the region we mapped with Spitzer. There are legitimate Taurus members outside our region, including, e.g., those in the L1551 region.

The core of our sample of previously identified Taurus members is the list assembled by the XEST team for their analysis (see Güdel et al. 2007b and references therein). We have updated this list with more recently confirmed objects (e.g., Scelsi et al. 2008), as well as scattered additional previously identified Taurus members found in the literature. Kenyon et al. (2008) also report previously identified Taurus objects, with $\sim 30$ more objects in our Spitzer field of view, which we have also included (but see Appendix B.7 for one object from Kenyon et al. (2008) which we rejected). We have thus defined our sample of previously identified Taurus members as basically an updated XEST+Kenyon et al. list convolved with our survey coverage. There are 215 previously identified Taurus members in our Spitzer maps. There are $\sim 100$ more objects from Kenyon et al. which are outside our mapped region. Binary objects that are unresolved in any of our Spitzer maps are regarded here as a single object (e.g., FS Tau Aab, whose separation is $<1^{\prime \prime}$ ). We discuss the overall Spitzer properties of this list in some detail below. We note here that all of these 215 previously identified Taurus members were detected by Spitzer, but not all of them have IR excesses; this list includes the young stars without IR excesses (e.g., mostly weak-lined T Tauri stars, WTTS) discovered by other means.

Hartmann et al. (2005) report IRAC observations of a set of previously identified members covered by IRAC guaranteed time observation (GTO) team observations. This region of the sky is also covered by our shallower map, to the same depth as the rest of our survey. Our photometry agrees within the errors expected from photometry methodology and from the intrinsic variability of the stars.

We do not include the objects reported by Luhman et al. (2006, 2009a, 2009b) as previously known objects because they were found with an independent analysis of the same data used here-our Taurus Legacy data, in part along with the XEST data - and the derived values agree.

Scelsi et al. (2008) present spectroscopic follow-up on potential new Taurus members discovered by the XEST survey (Scelsi et al. 2007). Three confirmed new Taurus members from Scelsi et al. (2008) were independently discovered and confirmed by us using Spitzer data (SST Tau 043456.9+225835 = XEST 08003, SST Tau 043542.0+225222 = XEST 08-033, and SST Tau $042215.6+265706=$ XEST 11-078). Since we report these in the list of new Taurus members, these do not appear in the list of previously identified Taurus members; they are noted in the tables below. The new members reported there that we did not rediscover are included in our list of previously identified Taurus members. A complete discussion of the Spitzer properties of all of the candidate members found using XEST X-rays that were presented by Scelsi et al. (2007) will appear in M. Audard et al. (2010, in preparation).

\subsubsection{Template Sample of Non-members}

Aside from previously identified Taurus members, there are a large variety of other previously identified objects in our survey region. Many of these are clearly not Taurus members, but some are more ambiguous. The previously identified objects include known extragalactic objects, named objects of unknown nature, confirmed non-members, and potential (unconfirmed) members of Taurus.

To construct this list, we first searched in SIMBAD over our entire field to obtain a list of $\sim 8000$ known objects. For objects that did not already have high-precision coordinates, we went back to the original article reporting the discovery of the object and attempted re-identification of the object using finding charts and 2MASS images. If no finding charts were available, the brightest close object from 2MASS was assigned to the object's name. Some objects are not recoverable, but most were identified; nearly $90 \%$ of the entire 8000-object list has highaccuracy coordinates in the end. New coordinates were reported back to the SIMBAD team for inclusion in their database.

We also included the results from several papers from the literature reporting specifically confirmed non-members. These confirmed non-members can be candidate member objects from other Taurus surveys such as Luhman et al. (2006) or Scelsi et al. (2008), that failed a spectroscopic test for membership. They can also be spectroscopically confirmed background giants from studies of the ISM (e.g., studies of the Taurus dark cloud). These confirmed non-members have not necessarily been ingested into SIMBAD, since they were not the primary scientific result of the paper. Note that we did not list as non-members those objects merely assumed but not confirmed (via spectroscopy) to be background giants.

We merged this list by position with our master catalog to identify objects seen in our survey. For each object for which a match was found in our catalog, we went back to the original literature in an attempt to identify it as a known extragalactic object, a named object of unknown nature (e.g., objects from an all-sky survey where no specific follow-up has been done), a confirmed non-member (as defined immediately above), or a potential (unconfirmed) member of Taurus. In the case of objects from the literature listed as potential but unconfirmed Taurus members, we noted and book kept these objects separately, and we mention them where relevant below; some are indeed recovered here by our Spitzer-based searches for YSOs.

Thus, the sample of Taurus non-members is certainly biased and far from comprehensive and is defined to include mixtures of stars, other Galactic objects (such as planetary nebulae), and extragalactic objects. This sample can be indicative of some typical colors to expect from a variety of types of IR-bright non-member objects.

As a further diagnostic for non-members (including extragalactic objects), we merged by position to the 2MASS extended object catalog. Objects in this catalog are likely but not guaranteed to be all extragalactic objects-11 (out of 215) previously identified Taurus members are also 2MASS extended objects (due to, e.g., scattered light from extended dust structures), but 
107/148 previously known galaxies are 2MASS extended objects. 2MASS extended object identifications, if relevant, are noted in the data tables and SEDs below.

\subsubsection{The Process}

In order to find new candidate Taurus members, we first examined various color-color and color-magnitude spaces using our entire Taurus catalog, highlighting the locations of the previously identified objects (both members and non-members). We compared these diagrams to discussions in the literature also seeking to identify YSOs from Spitzer photometric measurements (e.g., Allen et al. 2004; Padgett et al. 2008b; Rebull et al. 2007; Harvey et al. 2007; Gutermuth et al. 2008). There is no single color selection criterion that is $100 \%$ reliable in separating members from non-member contaminants. Exactly which color selection criteria work best can be a strong function of the relative bandpass sensitivities and saturations, since 2MASS, IRAC, and MIPS do not all detect the same faintest objects (due not only to sensitivities but also degree of interstellar reddening and embeddedness of the young protostellar objects), or saturate for the same brightest objects. After extensive empirical investigation using diagrams from the literature as well as new diagrams, we selected four CMDs and one color-color diagram (CCD) which provided the best diagnostics for YSOs, and we used them to construct an initial list of new candidate YSOs. In each diagram, we define regions most likely to harbor YSO candidates, and regions most likely to contain galaxies or other non-members; these are listed in detail in Section 3.1.5.

By imposing these color selections, we are selecting objects that have IR excesses (e.g., flux densities above that expected for a photosphere) and whose overall brightness is consistent with objects at the Taurus distance. We interpret these excess objects as dusty objects, with circumstellar disks and/or envelopes. We do not select objects without IR excesses.

One aspect of our survey which makes it different from many of the Spitzer-based surveys in the literature is our extensive optical imaging. While the SDSS and CFHT imaging data do not cover every square arcminute of the Spitzer maps, they cover most of it. The SDSS spatial resolution is only slightly better than the IRAC resolution at $\sim 1$ ". 4 , but the CFHT data have much better spatial resolution at $\sim 0^{\prime}{ }^{\prime} 6-00^{\prime \prime} 8$. We examined the images at all available bands for each of the nearly 900 objects meeting the color-color or color-magnitude criteria (plus many more objects in the process of establishing these color spaces). At any band, if the object is a resolved galaxy, or projected in the vicinity of a galaxy cluster, we dropped it from further consideration. Some of the objects that are resolved are actually previously identified YSOs. Many of the objects that have Spitzer colors similar to YSOs turn out to be resolved galaxies when examined with SDSS or CFHT. These optical imaging data have been crucial to our ability to distinguish galaxies from YSO candidates.

If the candidate object meets the color criteria in any one of the color-magnitude spaces we investigated and passes the imaging/spatial resolution test, we regard it as a provisional YSO candidate, pending additional scrutiny discussed below. Objects meeting the color criteria but failing the imaging/ spatial resolution test are "candidate non-members" and appear separately in the figures below.

\subsubsection{Gradations of Confidence for YSO Candidates}

Previously identified Taurus members tend to be bright, because previous IR (and optical) surveys were shallower than our surveys. True new Taurus members are also likely to be generally bright. Very red (embedded or cool) objects could also be members, especially since this survey goes fainter in the IR than any prior survey of the region (excepting the Spitzer GTO observations in the various core regions; Hartmann et al. 2005). However, the fainter objects are also statistically more likely to be galaxies, especially over our survey area of more than $44 \mathrm{deg}^{2}$ at $-15^{\circ}$ galactic latitude. Thus, we specifically focused our attention on bright and/or red objects meeting our color selection criteria. Faint red objects meeting our color selection criteria were also considered but are statistically more likely to be galaxies than YSO Taurus members.

In addition to the easily quantifiable Spitzer magnitude and color criteria, we also individually assessed each candidate YSO using qualitative judgments. These include but are not limited to: morphology in imaging data in each available band; relative brightness at all bands from $U$ to $160 \mu \mathrm{m}$ (e.g., IR excess, but optical too bright to be a Taurus member); amplitude of excess; shape of SED; apparent (projected) proximity to other previously identified Taurus members; apparent (projected) proximity to clearly identifiable galaxies (e.g., appearing to be part of a galaxy cluster); resolvable spiral arms or tidal tails; previous identifications (e.g., with the 2MASS extended source catalog); estimated $A_{V}$ from the $160 \mu \mathrm{m}$ map (e.g., objects seen in high extinction regions are likely Taurus members); and star counts (a similar criterion to proximity to galaxies or estimated $\left.A_{V}\right)$. These assessments were done over several weeks by groups of co-authors and resulted in increased appreciation of the range of contaminants, and more objects being identified as new likely galaxies. (See the appendix discussion on " $8 \mu \mathrm{m}$ pop-up objects," Appendix B.7, for an example of a class of objects we rejected.) We looked critically at the shape of the excess above the photosphere, and if the excess appeared only at one band (8 or $24 \mu \mathrm{m}$ ), we retained the object as a YSO candidate only if it was more than $4 \sigma$ above the photosphere (see Appendix B.5 for many of the rejected low-significance sources). For the surviving YSO candidates, based on all of the available information from any wavelength (spectroscopic as well as photometric, plus derived information such as placement in a theoretical Hertzsprung-Russell (HR) diagram-see Section 4.6) as well as all the criteria listed above, we assigned a letter grade, A/B / $\mathrm{C}$, with grades of "A" as more likely members than those with grades of "C."

These qualitative criteria can fail to recover some of the previously identified Taurus members. Several of the previously identified Taurus members do not have IR excesses and are therefore not recoverable by our search. Seven of the previously identified Taurus members (e.g., $042146.3+265929$ or $042307.7+280557$ ) would probably have been rejected because in the optical images, these objects are in front of a field of galaxies, i.e., they appear to be part of a galaxy cluster, and are not in a high $A_{V}$ region. One additional previously identified member, the well-known edge-on disk IRAS 04302+2247 (= SST Tau $043316.5+225320$ ), was temporarily identified as a galaxy because its appearance was so unusual in the optical image. While our process is clearly imperfect, we are confident that, working as a group and using all of the available multiwavelength information, we have identified a reasonably high-confidence sample of candidate Taurus members present in our photometric catalog.

Our approach for finding YSOs is customized to our data set. This labor-intensive process is not one that can be blindly applied to other regions, even regions where similar extensive supporting optical data are available. While our color selection 
Table 1

Sample Selection Criteria

\begin{tabular}{|c|c|c|}
\hline Sample Selected via & YSO Selection & Faint Flag \\
\hline 24/70 CMD & Either $[24]<7$ OR $[24]-[70]>6$ & {$[24]>7$} \\
\hline$K_{\mathrm{s}} / 24 \mathrm{CMD}$ & $K_{\mathrm{s}}<14$ AND $K_{\mathrm{s}}-[24]>1$ & $K_{\mathrm{S}}>13.5$ \\
\hline 8/24 CMD & {$[8]-[24]>0.5$ AND } & {$[8]>9.5$} \\
\hline 4.5/8 CMD & $\begin{array}{l}\quad(([8]-[24]<4 \text { and }[8]<10) \text { or }([8]-[24] \geqslant 4 \text { and }[8]<2.5 \times([8]-[24]))) \\
{[4.5]<6 \text { AND }([4.5] \geqslant 6 \text { and }[4.5] \leqslant 11.5} \\
\text { and }[4.5]-[8]>0.4) \text { OR }([4.5]>11.5 \text { and }[4.5]<0.6944 \times([4.5]-[8])+11.22)\end{array}$ & {$[4.5]>11$} \\
\hline IRAC CCD & {$[3.6]-[4.5]>0.15$ and $[5.8]-[8]>0.3$ and $[3.6]<13.5$} & . \\
\hline
\end{tabular}

can be easily applied to any Spitzer+2MASS catalog, the manual examination of each object is not necessarily easily duplicated and certainly automating this process is not currently possible. However, because our survey is wide-area, and the contamination rate is high, this process is unavoidable and has been crucial to our YSO selection. The time we spent in vetting the candidate list enabled more efficient use of our follow-up spectroscopic telescope time, e.g., there was little time wasted in taking spectra of contaminants.

\subsubsection{The Figures and Sample Selection Criteria}

The color-color and color-magnitude spaces we have chosen to use (see Section 3.1.3 for overview) are the following: [24] versus [24]-[70] (Section 3.2.1), $K_{\mathrm{s}}$ versus $K_{\mathrm{s}}-$ [24] (Section 3.2.2), [8] versus [8]-[24] (Section 3.2.3), [4.5] versus [4.5]-[8] (Section 3.2.4), and finally, [3.6]-[4.5] versus [5.8]-[8] with an additional [3.6] brightness cutoff (Section 3.2.5). Table 1 summarizes the details of the sample selection criteria for each parameter space. Our final selection includes objects selected in any of these parameter spaces (not just objects selected in all of them); this will be discussed in more detail in Section 4.1. (We explicitly compare this selection method to others from the literature in Section 4.7 below.) We discuss each of these parameter spaces, in the order given above and in Table 1, in Sections 3.2.1-3.2.5; for each, there is a figure (Figures 1-5) consisting of six panels. Each of the panels contains either a subsample or a comparison sample to clearly demonstrate our selection techniques. In the remainder of this section, we discuss each of the panels in introductory terms only.

In the upper left of each figure is the SWIRE (Spitzer Widearea Infrared Extragalactic Survey; Lonsdale et al. 2003) ELAIS N1 extragalactic field ${ }^{23}$ (the c2d reduction-see Evans et al. 2007-is used here, as in Rebull et al. 2007 and Padgett et al. 2008b). The ELAIS N1 field is a $\sim 6 \mathrm{deg}^{2}$ field centered on $16^{\mathrm{h}} 08^{\mathrm{m}} 44^{\mathrm{s}}+56^{\mathrm{d}} 26^{\mathrm{m}} 30^{\mathrm{s}}(\mathrm{J} 2000)$, or galactic coordinates $(l, b)$ of $86.95,+44.48$ (to be compared with the Taurus map center of $l, b \sim 173,-15)$. The SWIRE sample is expected to be essentially entirely galaxies and foreground stars, and as such provides a visual guide to the locations where such objects appear in the corresponding diagram. Note that this is just the $\sim 6 \mathrm{deg}^{2}$ field, as observed; it has not been scaled up to represent $\sim 44 \mathrm{deg}^{2}$ of Taurus data, because in this case we are primarily interested in the range of colors sampled by the galaxies, not the overall numbers. As we will see below, many newly discovered extragalactic objects in our survey have colors very similar to many certifiable YSOs, and different than the colors of objects found in SWIRE. Note also the Galactic latitude difference; this difference in Galactic latitude is likely to dominate the source

\footnotetext{
23 VizieR Online Data Catalog, II/255 (J. Surace et al. 2004).
}

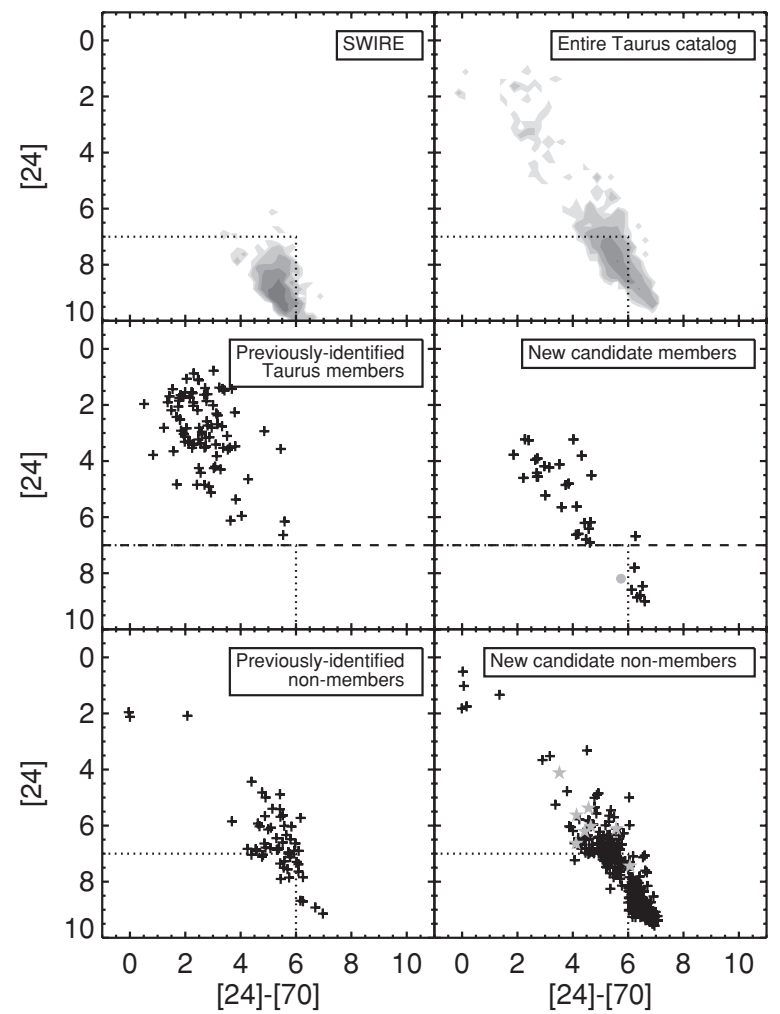

Figure 1. [24] vs. [24]-[70] for (top) the SWIRE sample (essentially all galaxies; contours at $1,2,4,8$, and 16 objects), the entire Taurus sample (YSOs+contaminants; contours at 1,2,5,15, and 35; dotted line indicates region considered for YSO candidacy), (middle) the sample of previously identified Taurus members, and the sample of all new candidate members $(+=$ objects selected in this color-magnitude space; gray dots $=$ objects selected based on other color-magnitude spaces; dotted line indicates region considered for YSO candidacy; dashed line indicates cutoff for "faint" flag), and (bottom) the sample of previously identified non-members (stars and galaxies) and the sample of new candidate non-members (stars and galaxies) $(+=$ objects selected in this colormagnitude space; gray stars $=$ objects selected as YSOs but spectroscopically confirmed to be non-members; dotted line indicates region considered for YSO candidacy).

counts in IRAC bands 1 and 2. More discussion of relative source counts will appear in P10.

In the upper right panel of each figure, our entire Taurus catalog is represented, so that various subsamples can be seen in the context of the larger catalog. The Taurus catalog is expected to consist of YSOs, foreground/background stars (and other non-stellar galactic objects such as planetary nebulae), and background galaxies (recall that the asteroids have already been removed). To first order, then, the objects in the Taurus catalog that do not resemble the objects found in SWIRE are the YSOs. However, the populations are not necessarily well separated, as can be seen in the remaining panels of the figures. 


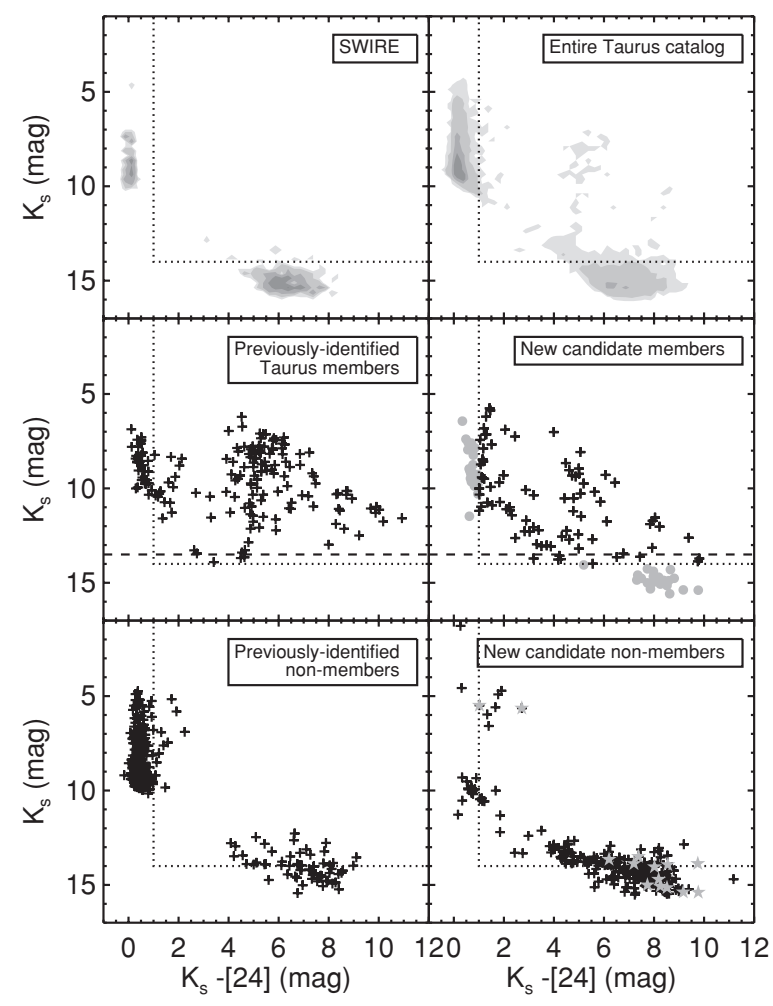

Figure 2. $K_{\mathrm{s}}$ vs. $K_{\mathrm{s}}-$ [24] for (top) the SWIRE sample (galaxies and foreground stars; contours at $1,2,4,8$, and 16 objects), the entire Taurus sample (YSOs+contaminants; contours at 1, 5, 50, 100, and 200 objects; dotted line indicates region considered for YSO candidacy), (middle) the sample of previously identified Taurus members, and the sample of all new candidate members $(+=$ objects selected in this color-magnitude space; gray dots $=$ objects selected based on other color spaces; dotted line indicates region considered for YSO candidacy; dashed line indicates cutoff for "faint" flag), and (bottom) the sample of previously identified non-members (stars and galaxies) and the sample of new candidate non-members(stars and galaxies) $(+=$ objects selected in this color-magnitude space; gray stars = objects selected as YSOs but spectroscopically confirmed to be non-members; dotted line indicates region considered for YSO candidacy).

The remaining four panels in each figure are subsets of the Taurus catalog. The second row of plots contains YSOs, both the previously identified Taurus members (left) and new candidate Taurus members (right), those selected by that particular diagram (+) as well as others selected from other diagrams (gray dots). The distribution of previously identified Taurus members includes those selected based on IR excess and those selected via other mechanisms, and thus includes objects without IR excesses; of course, we will not find objects like the latter using Spitzer. Note also that the distribution of previously identified Taurus members often includes objects that have colors resembling galaxies. This is not surprising, since the galaxies are indeed undergoing star formation; thus, these color selection mechanisms are successfully finding star formation, just not necessarily in Taurus. The set of new candidate Taurus members is constructed from a color and magnitude cut on the entire sample, and then examining all of the available data for each of the candidates, dropping the likely galaxies (Section 3.1.4).

The third and final row contains the distributions for nonmembers, both previously identified and newly identified here. The left panel is the sample of previously identified nonmembers which, as discussed in Section 3.1.2, includes stars identified via proper motions, background giants, and galaxies identified in the literature. The last panel is the sample of all

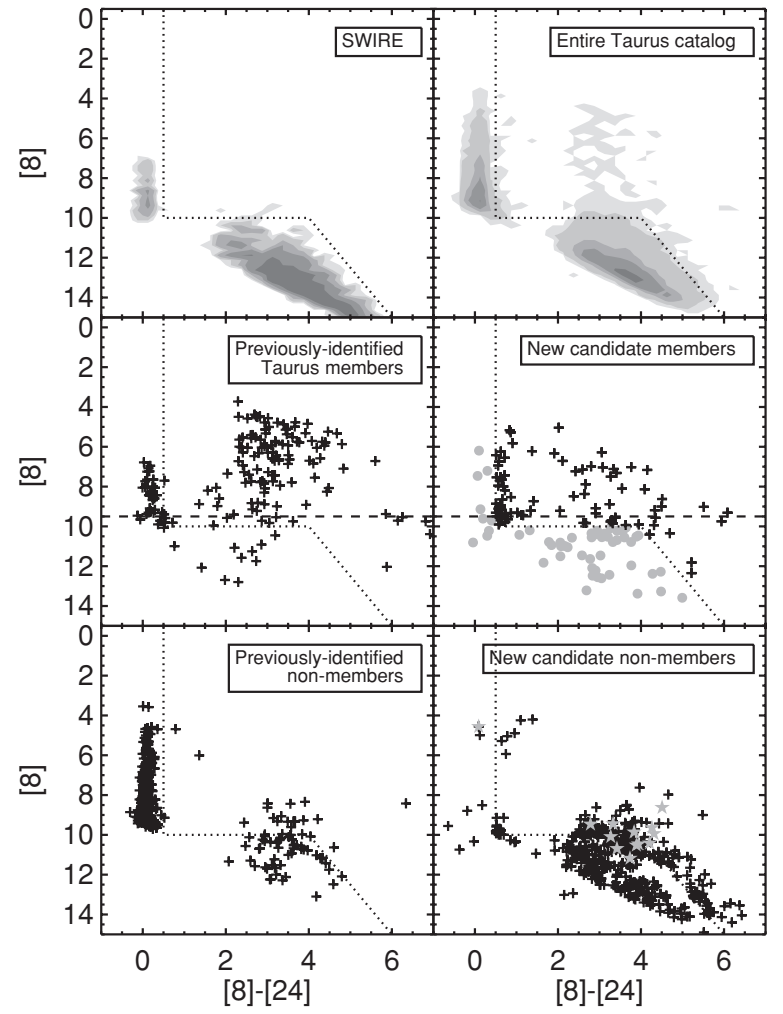

Figure 3. [8] vs. [8]- [24] for (top) the SWIRE sample (galaxies and foreground stars; contours at $1,2,4,8$, and 16 objects), the entire Taurus sample (YSOs+contaminants; contours at 1, 5, 50, 100, and 200 objects), (middle) the sample of previously identified Taurus members, and the sample of all new candidate members $(+=$ objects selected in this color-magnitude space; gray dots $=$ objects selected based on other color spaces; dotted line indicates region considered for YSO candidacy; dashed line indicates cutoff for "faint" flag), and (bottom) the sample of previously identified non-members (stars and galaxies) and the sample of new candidate non-members (stars and galaxies) $(+=$ objects selected in this color-magnitude space; gray stars = objects selected as YSOs but spectroscopically confirmed to be non-members; dotted line indicates region considered for YSO candidacy).

objects identified as possibly YSOs based on Spitzer colors but then rejected as such, based primarily on inspection of the optical images and SEDs; see Section 3.1.4. Objects which passed all the other tests to be YSO candidates but failed the spectroscopic test (see Section 2.3) are indicated in the last two panels of the figures by gray stars. (Note that, having been selected by the other tests, they also appear in the fourth panel as candidate YSOs.)

Thus, for the color-magnitude or color-color space represented by each figure, one can examine and compare the distribution of galaxies (SWIRE, previously identified non-members, new non-members), the distribution of YSOs (previously identified Taurus members), the distribution of foreground/ background stars (SWIRE, previously identified non-members), and the distribution of new candidate Taurus members.

\subsection{Implementation of the Spitzer Selection Criteria}

\subsubsection{Selection via [24] versus [24]-[70]}

The [24] versus [24]-[70] diagram has been used before to find new candidate YSOs (e.g., Padgett et al. 2008b; Rebull et al. 2007). Figure 1 shows this CMD for the six samples mentioned in Section 3.1.5 above (left to right, top to bottom): SWIRE (expected to be essentially entirely galaxies), the entire Taurus sample, previously identified Taurus members, new 


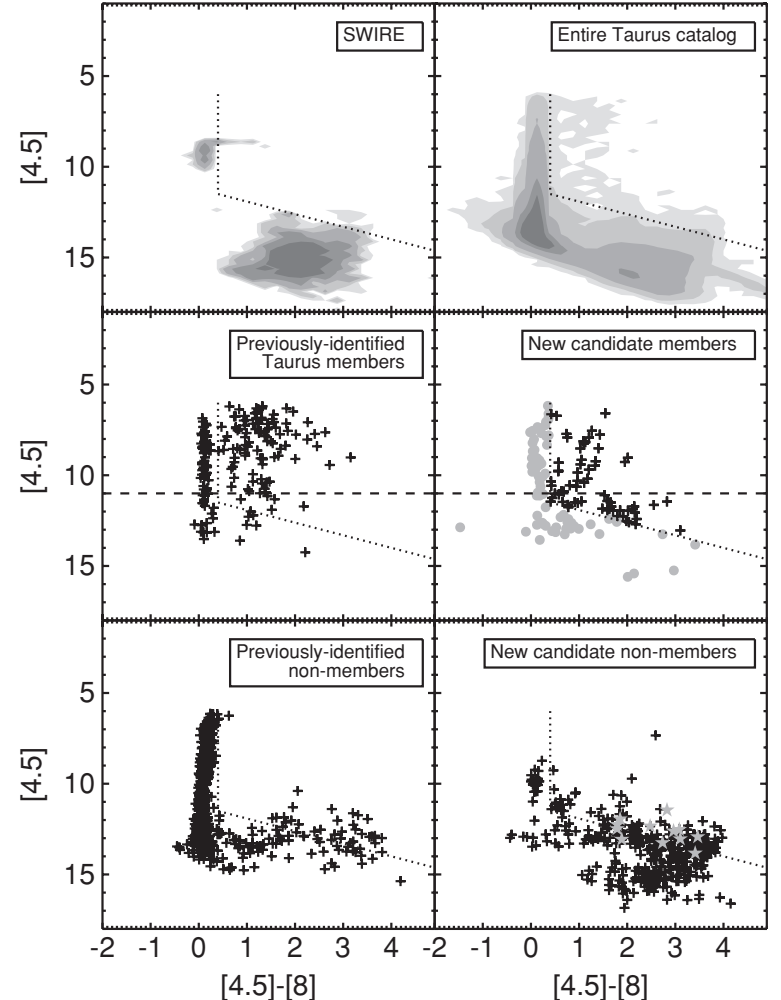

Figure 4. [4.5] vs. [4.5]-[8] for (top) the SWIRE sample (galaxies and foreground stars; contours at $1,2,4,8,16$ objects), the entire Taurus sample (YSOs+contaminants; contours at 1, 10, 100, 1000, 2000 objects; dotted line indicates region considered for YSO candidacy), (middle) the sample of previously identified Taurus members, and the sample of all new candidate members $(+=$ objects selected in this color-magnitude space; gray dots $=$ objects selected based on other color spaces; dotted line indicates region considered for YSO candidacy; dashed line indicates cutoff for "faint" flag), and (bottom) the sample of previously identified non-members (stars and galaxies) and the sample of new candidate non-members (stars and galaxies) $(+=$ objects selected in this color-magnitude space; gray stars = objects selected as YSOs but spectroscopically confirmed to be non-members; dotted line indicates region considered for YSO candidacy).

candidate Taurus members, previously identified non-members (stars identified via proper motions, background giants, and galaxies identified in the literature), and new candidate nonmembers identified here.

By inspection of Figure 1, we find that objects with [24] > 7 and $[24]-[70]$ between about four and seven are statistically likely to be galaxies. Unadorned photospheres (e.g., old foreground stars) will be bright and have [24]-[70] 0; an A3 ZAMS photosphere has [24] 7 at the distance of Taurus, and for a median Taurus-age member, [24] $\sim 7$ corresponds to midK. Compared to the SWIRE catalog, the entire Taurus catalog contains many objects with similar colors, but also many objects that are similarly red and much brighter at [24], and therefore are candidate dusty young stars.

Based on the properties of the previously identified member and non-member samples, the properties of the SWIRE sample, and discussions in the literature, the selection we impose to search for new candidate YSOs is either [24] $<7$ or [24]-[70] $>6$. Statistics on this sample are given in Table 2 (along with statistics from the SWIRE sample for comparison); in summary, this cut yields $\sim 450$ objects, each of which we investigated at all our available imaging bands; $~ 20 \%$ of them are previously identified Taurus members, $\sim 7 \%$ of them survive the tests to be potential new YSOs, and $\sim 70 \%$ are

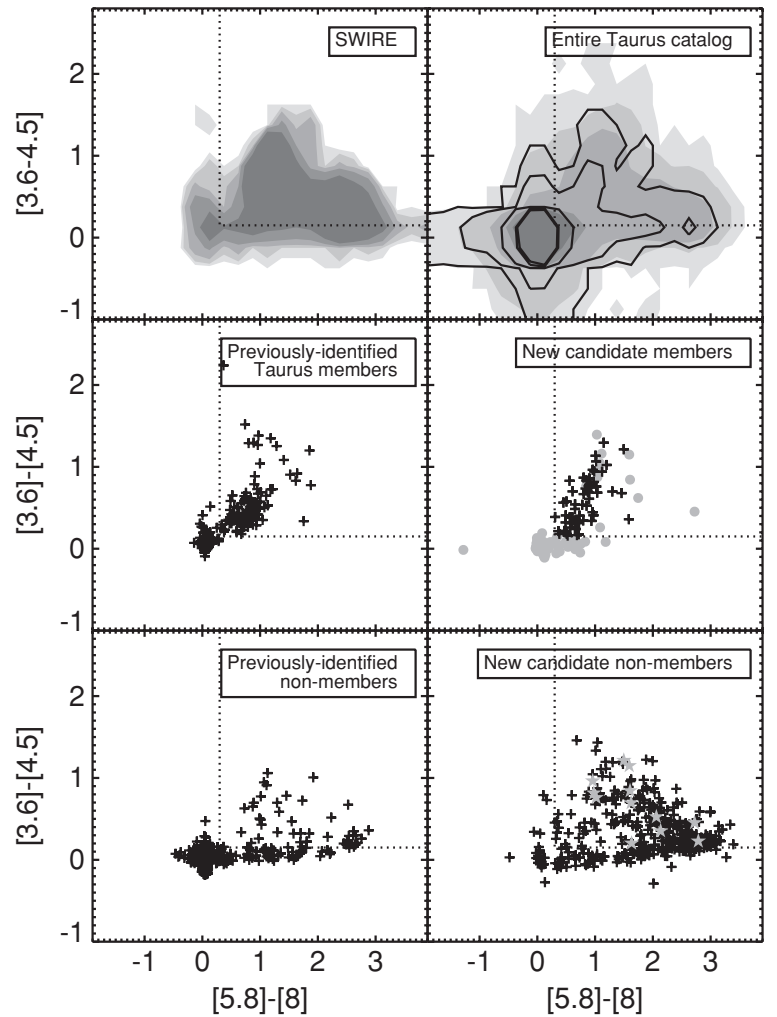

Figure 5. [3.6]-[4.5] vs. [5.8]-[8] for (top) the SWIRE sample (galaxies and foreground stars; contours at 1, 2, 4, 8, and 16 objects), the entire Taurus sample (YSOs+contaminants; gray contours at 1, 10, 100, 1000, and 2000 objects; solid line contours are for the entire Taurus sample with an additional [3.6] $<13.5$, same contour limits as the gray contours), (middle) the sample of previously identified Taurus members, and the sample of all new candidate members ++ $=$ objects selected in this color-color space; gray dots $=$ objects selected based on other color spaces; dotted line indicates region considered for YSO candidacy), and (bottom) the sample of previously identified non-members (stars and galaxies) and the sample of new candidate non-members(stars and galaxies) $(+=$ objects selected in this color-color space; gray stars $=$ objects selected as YSOs but spectroscopically confirmed to be non-members; dotted line indicates region considered for YSO candidacy). An additional [3.6] brightness cut was also imposed on the YSO selection in this color space; see the text.

previously identified or new non-member objects. Nearly all of the previously identified Taurus members that appear in this plot have [24] $<7$. This is a likely bias in that all of the previous IR surveys searching for Taurus members were much shallower than this Spitzer survey-the IRAS sensitivity limit was about $0.3 \mathrm{Jy}$, so we are going $7 \mathrm{mag}$ fainter than IRAS. However, in order to appear in this plot, the objects have to have been detected at $70 \mu \mathrm{m}$ as well, so the sensitivity of the $70 \mu \mathrm{m}$ survey is usually the limiting factor. Of the entire sample of previously identified Taurus members within our survey, $80 \%$ are detected at $24 \mu \mathrm{m}$ (see Table 3), and just $45 \%$ are detected at $70 \mu \mathrm{m} ; \sim 5 \%$ of the previously identified Taurus members are saturated in MIPS-24 and 3\% are saturated in MIPS-70.

In this diagram, the set of previously identified Taurus objects is generally distinguished from the distribution of faint objects found in the SWIRE sample. Fainter Taurus objects $([24]>7)$ could exist, but objects that faint are statistically likely to be galaxies; their properties at other bands could suggest otherwise. Objects surviving the imaging test (and other qualitative criteria—see Section 3.1.4) but with [24] $>7$ are therefore further identified as "faint." About 17\% of the 35 potential new YSOs in this parameter space are faint. Many of the other faint objects selected by our color/magnitude cut 
Table 2

Sample Properties I

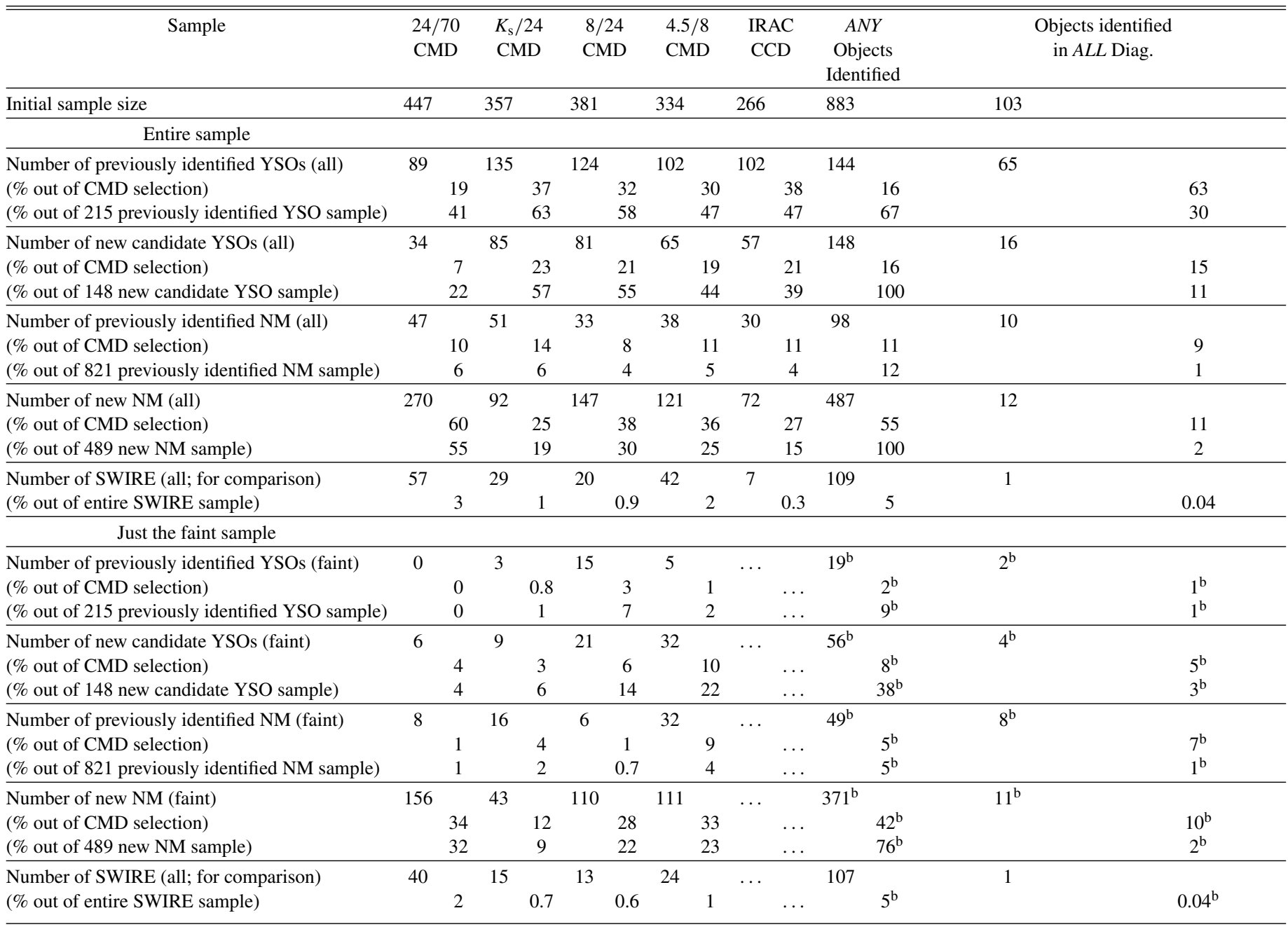

Notes.

a As an example for how to read this table, in the case of the 24/70 CMD, 447 objects are selected by our color cuts in this diagram. Of these, $19 \%$ are previously identified YSOs, $7 \%$ are new candidate YSOs, $10 \%$ are previously identified non-members, and $60 \%$ are new non-members. And, out of the 215 stars that compose our previously identified Taurus member sample, $41 \%$ are recovered in the 24/70 diagram, of the 148 objects in our candidate YSO sample, $22 \%$ are found here, of the 821 previously identified non-members, $6 \%$ are found in this diagram, and of the 489 new non-members, $55 \%$ are found here.

${ }^{\mathrm{b}}$ Objects listed here were picked in any diagram as being faint.

indeed resolve into galaxies when examined using the CFHT or SDSS imaging — $60 \%$ of all of the objects selected in this space are new galaxy candidates. About 100 of the brighter objects selected by this color cut resolve into galaxies, so faintness alone is insufficient for locating and identifying galaxies. As can be seen in Figure 1, most of the previously identified nonmembers and new candidate non-members resemble the colors of objects found in SWIRE. Figure 1 and the statistics in Table 3 also demonstrate that our sample of new candidate YSOs is on average redder and fainter than the sample of previously identified YSOs.

As discussed above (Section 2.3), we have obtained Palomar and/or Keck spectroscopy of many of our candidate objects. Because this color selection uses bandpasses far from optical, these objects are often very faint indeed at optical or NIR bands. We have spectroscopy for about $70 \%$ of the 34 new candidate YSOs selected in this color space. So far, almost $90 \%$ of those are stellar (e.g., YSOs or stars that could still be shown to be foreground stars or background giants), and just four are confirmed to be extragalactic objects.

\subsubsection{Selection via $K_{s}$ versus $K_{s}-[24]$}

As for [24] versus [24]-[70], the $K_{\mathrm{s}}$ versus $K_{\mathrm{s}}-$ [24] diagram has been used previously to find new candidate YSOs (e.g., Padgett et al. 2008b; Rebull et al. 2007). Figure 2 shows this CMD for the same samples as Figure 1 (see Section 3.1). The SWIRE sample clearly (more obviously than the previous diagram) consists of both galaxies $\left(K_{\mathrm{S}} \gtrsim 14\right.$ and $K_{\mathrm{S}}-[24]$ between about 4 and 8$)$ and stars $\left(K_{\mathrm{s}} \lesssim 10\right.$ and $\left.K_{\mathrm{s}}-[24] \sim 0\right)$. As before, the entire Taurus catalog has many objects with colors similar to the SWIRE sample, but also many objects that have properties different than the SWIRE sample, e.g., redder than $K_{\mathrm{s}}-$ [24] $\sim 1$ and brighter than $K_{\mathrm{s}} \sim 14$, as well as redder than $K_{\mathrm{s}}-[24] \sim 8$. Note that the lack of sources in the lower left of each panel is an artifact of the sensitivity limits of the survey. 
Table 3

Sample Properties II

\begin{tabular}{lccrc}
\hline \hline \multicolumn{1}{c}{ Property } & $\begin{array}{c}\text { Prev. Ident. YSOs } \\
\text { Total (Fraction) }\end{array}$ & $\begin{array}{c}\text { Prev. Ident. YSOs, Bright }{ }^{\mathrm{c}} \\
\text { Total (Fraction) }\end{array}$ & $\begin{array}{c}\text { New Candidate YSOs } \\
\text { Total (Fraction) }\end{array}$ & $\begin{array}{c}\text { New Candidate YSOs, Bright } \\
\text { Total (Fraction) }\end{array}$ \\
\hline Total sample size & $215(1.00)$ & $196(1.00)$ & $148(1.00)$ & $92(1.00)$ \\
Having IRAC $3.6 \mu \mathrm{m}$ & $193(0.90)$ & $174(0.89)$ & $142(0.96)$ & $86(0.93)$ \\
Having IRAC $4.5 \mu \mathrm{m}$ & $189(0.88)$ & $170(0.87)$ & $145(0.98)$ & $90(0.98)$ \\
Having IRAC 5.8 $\mu \mathrm{m}$ & $212(0.99)$ & $193(0.98)$ & $147(0.99)$ & $91(0.99)$ \\
Having IRAC $8.0 \mu \mathrm{m}$ & $206(0.96)$ & $187(0.95)$ & $147(0.99)$ & $92(1.00)$ \\
Having MIPS $24 \mu \mathrm{m}$ & $173(0.80)$ & $154(0.79)$ & $135(0.91)$ & $82(0.89)$ \\
Having MIPS 70 $\mu \mathrm{m}$ & $95(0.44)$ & $89(0.45)$ & $35(0.24)$ & $17(0.18)$ \\
Having all four IRAC & $187(0.87)$ & $168(0.86)$ & $141(0.95)$ & $86(0.93)$ \\
Having all four IRAC+MIPS 24 & $154(0.72)$ & $135(0.69)$ & $128(0.86)$ & $76(0.83)$ \\
Having both MIPS & $89(0.41)$ & $83(0.42)$ & $35(0.24)$ & $17(0.18)$ \\
Having $K_{\mathrm{S}}$ & $209(0.97)$ & $194(0.99)$ & $140(0.95)$ & $92(1.00)$ \\
Having prior name & $215(1.00)$ & $196(1.00)$ & $65(0.44)$ & $46(0.50)$ \\
\hline
\end{tabular}

Notes.

${ }^{a}$ Previously identified YSO Taurus members.

${ }^{\mathrm{b}}$ Notation indicates in each case that the first number is the total number in that (sub)sample, and the second number (in parentheses) is the sample fraction, e.g., 192 out of 215 previously identified Taurus members have $3.6 \mu \mathrm{m}$ measurements, or $90 \%$ of the 215 star sample.

${ }^{\mathrm{c}}$ Previously identified YSO Taurus members, bright subsample, e.g., those not flagged as red and faint; see Section 3.1.4. The purpose of extracting the bright sample as distinct from the entire sample is to demonstrate the properties of the brightest objects, e.g., in the case of the new candidate Taurus members, those least statistically likely to be galaxies.

The sample of previously identified Taurus members, for the most part, have $K_{\mathrm{s}}<14$, which generally avoids the region populated by galaxies in the SWIRE sample, but there are legitimate YSOs mixed in with the galaxies in this parameter space. Here too, the historical bias toward brighter objects in prior surveys can be seen, and faint red objects could be legitimate YSOs. Essentially all of the previously identified YSO sample has a $K_{\mathrm{s}}$ measurement in our database, but as mentioned above, just $\sim 80 \%$ are cleanly detected at $24 \mu \mathrm{m}$ (see Table 3). As above, fainter objects are statistically likely to be galaxies.

The selection we impose on this parameter space to search for new candidate YSOs is that $K_{\mathrm{s}}<14$ and $K_{\mathrm{s}}-[24]>1$. Again, Table 2 summarizes the sample sizes; $\sim 360$ objects meet these criteria, including most of the (detected) previously identified Taurus members; note that there are some stars without apparent IR excess (e.g., likely WTTS) with $K_{\mathrm{s}}-[24] \sim 0$, and there are YSOs that have colors resembling galaxies. Note also that late-type stars do not have $K_{\mathrm{s}}-[24]=0$ (Gautier et al. 2007). Objects with $K_{\mathrm{S}}>13.5$ are further identified as "faint" and thus statistically likely to be galaxies. Previously identified Taurus members compose 135 of the objects meeting the basic color criteria; about 50 are previously identified non-member objects, most of which are 2MASS extended sources (which could be galactic or extragalactic objects). By inspection of the individual images, about 100 of the objects selected here are clearly resolved galaxies. Besides the previously identified Taurus objects, 85 objects are indistinguishable from point sources, or have morphologies consistent with YSO candidates, and meet all the other qualitative criteria (see Section 3.1.4) for potential new YSOs selected via this color space; a quarter of these were already found via the 24/70 CMD above.

In this parameter space, there is still a bias (relative to the previously identified Taurus member sample) toward finding red and faint objects, but this appears to be not nearly as strong as it was in the $24 / 70$ space above. Of the 85 candidate YSOs found in this space, we have already obtained Palomar and/or Keck spectroscopy (see Section 2.3) for 85\% of them. All of them except for one are stellar (e.g., YSOs or stars that could still be shown to be foreground stars or background giants); just one is rejected outright as a galaxy.

A relatively high fraction of the literature background giants appear as selected in this parameter space. Because there is a large difference in wavelength between $K_{\mathrm{s}}$ and [24], this search is particularly sensitive to objects with small excesses, which could be interesting transition disk candidates. However, these objects could also be subject to reddening from the Taurus cloud that is high enough to significantly affect $K_{\mathrm{s}}$ but not $24 \mu \mathrm{m}$, or Taurus cloud emission affecting the $24 \mu \mathrm{m}$ but not the $K_{\mathrm{s}}$ photometrybackground giants are therefore potentially selected in this space. Several objects presented in the literature as candidate background objects based on shorter-wavelength photometric observations (i.e., without confirming spectroscopy) appear here as objects with potential excesses only at the longer wavelengths. With the information we have, we are unable to distinguish currently between transition disk candidates (i.e., Taurus objects with excesses only at $24 \mu \mathrm{m}$ ) and confirmed background giants. These objects are all identified in Table 5 as candidate non-members which we have promoted to lowgrade candidate YSOs. The SEDs that appear in Appendix A reveal that several of our candidate objects indeed have $K_{\mathrm{S}}$ values significantly affected by reddening and some candidate objects with clear cloud emission at 8 and/or $24 \mu \mathrm{m}$ are indicated in Table 7; also see Appendix B.5 for discussion of objects with very small excesses, usually just at $24 \mu \mathrm{m}$. We expect that several of the objects we have identified here will turn out to be background giants. Some candidate transition disk objects will be discussed in C.-E. McCabe et al. (2010, in preparation).

We note here that for surveys where the IRAC and MIPS coverage is well matched, using [3.6] or even [4.5] in place of $K_{\mathrm{s}}$ for this color-magnitude space is likely to be a better choice for searching for YSOs for two reasons: (a) minimizing the influence of reddening on $K_{\mathrm{s}}$ (3.6 or $4.5 \mu \mathrm{m}$ is less affected by reddening than $K_{\mathrm{s}}$; see Padgett et al. (2008b) for more discussion on the influence of $A_{V}$ ), and (b) minimizing the intrinsic range of star colors - the intrinsic $K_{\mathrm{s}}$ - [24] color of M stars is not zero (Gautier et al. 2007), whereas [3.6]-[24] or [4.5]-[24] is zero for those stars. Specifically for our survey, the overall $A_{V}$ toward 
Taurus is low, and all young stars at the distance of Taurus should be visible to 2MASS unless they are edge-on substellar objects. Moreover, using [3.6] or [4.5] in place of $K_{\mathrm{S}}$ does not reveal any YSO candidates not already selected by the color spaces used here, and finds in total only two more extragalactic objects. Had we used either [3.6] or [4.5] in place of $K_{\mathrm{s}}$, however, we would have found a factor of $\sim 4$ fewer objects that we believe (based on inspection and our qualitative criteria) to be likely reddened background giant contaminants.

\subsubsection{Selection via [8] versus [8]-[24]}

While essentially all of the previously identified YSOs have 2MASS detections at $K_{\mathrm{s}}$, some fainter legitimate YSOs may be embedded enough that the relatively shallow 2MASS survey will not detect the objects at $K_{\mathrm{s}}$, whereas they will be detected by our IRAC survey. Thus, we chose to investigate the [8] versus [8]-[24] parameter space; see Figure 3.

The morphology of this space is similar to the $K_{\mathrm{s}}$ versus $K_{\mathrm{s}}-[24]$ space, except the region occupied primarily by galaxies is now more elongated in color and has a more prominent slope toward fainter and redder objects. In order to select specifically for objects redder than most galaxies, the selection criteria we used consist of three line segments: (a) [8]-[24] $>0.5$ to avoid the stars without excesses; and (b) ([8]-[24] $<4$ and [8] $<10)$ to catch the bright stars in the middle of the plot; OR ([8]-[24] $\geqslant$ 4 and $[8]<2.5 \times([8]-[24]))$ to obtain the reddest stars. Objects with [8] $>9.5$ are statistically likely to be galaxies, and thus those are further identified as "faint" in Table 5.

About 380 objects in the catalog meet these criteria, $\sim 120$ of which are previously identified YSOs, $\sim 180$ of which are nonmembers (previously identified or new), and $\sim 80$ of which survive the imaging test (and other qualitative criteria - see Section 3.1.4) and remain potential YSOs. Of these, $\sim 25$ were already found using the [24] and [70] selection criteria (Section 3.2.1), $\sim 55$ were found using $K_{\mathrm{S}}$ and [24] (Section 3.2.2), and $\sim 20$ were found in all three color-magnitude planes.

There is an apparent gap in the last panel of Figure 3 inside the "galaxy blob" at [8]-[24] $>4$ and [8] $>10$. This is a direct result of our selection methodology. The fainter, bluer part of the distribution are largely those objects that were not obviously point sources in the optical imaging, and the redder part of the distribution closely tracks the (dotted) dividing line we used for our selection criteria. This population is composed of objects we rejected as candidate YSOs based on the qualitative criteria listed in Section 3.1.4 above.

We have follow-up spectroscopy (see Section 2.3) for $\sim 80 \%$ of the 81 new candidate YSOs selected in this color space. Nearly all (95\%) of these are stellar; just three are rejected as galaxies.

\subsubsection{Selection via [4.5] versus [4.5]-[8]}

To this point, we have required MIPS-24 detections for YSO candidate selection, which strongly biased our sample of new potential YSOs toward the generally brighter and/or larger excess objects (by comparison to the rest of the catalog). As an example of how many YSOs we may be missing by requiring $24 \mu \mathrm{m}$, just $\sim 80 \%$ of the previously identified YSOs are detected at $24 \mu \mathrm{m}$. This results from a combination of intrinsic disk properties (where disk emission makes the objects easier to detect at $24 \mu \mathrm{m}$ ) and the Spitzer sensitivity relative to low-mass photospheres at the Taurus distance (for those YSOs without disks). By loosening this restriction and not requiring MIPS-24, we extend the sample of potential objects, but also the potential contamination. We now investigate the [4.5] versus [4.5]-[8] parameter space; see Figure 4. This parameter space, on its own, provides the largest possible initial sample size we have yet investigated, as $17 \%$ of the entire catalog is detected in these two IRAC bands (compared with just $2 \%$ of the entire catalog detected at MIPS-24; see P10 for additional similar statistics). To first order, the morphology of this space is similar to the other spaces we have investigated, with the photospheres clustering around [4.5]-[8] 0 and the galaxies in a red and faint grouping. There are some new features apparent in this space, however. Saturation at $4.5 \mu \mathrm{m}$ occurs at $650 \mathrm{mJy}$ (6.1 mag), so the locus of colorless objects is truncated at that level. In the sample of previously identified Taurus members, there is a clear distinction between the disked and non-disked population (a gap near $[4.5]-[8]=0.5)$ which is not seen when considering the entire catalog.

The selection criteria we used to find candidate YSOs in this space selected the brighter and redder objects; we did this by stitching together several line segments. They are (a) [4.5] $<6$ AND (b) ([4.5] $\geqslant 6$ and [4.5] $\leqslant 11.5$ and $[4.5]-[8]>0.4)$ OR (c) $([4.5]>11.5$ and [4.5]< $0.6944 \times([4.5]-[8])+11.22)$. About 335 objects meet these basic criteria, $\sim 100$ of which are previously identified YSOs, and $\sim 160$ of which are new or previously identified nonmembers. About 65 objects survive the imaging test (and other qualitative criteria - see Section 3.1.4) and are new candidate YSOs. The distribution of these objects in this color-magnitude space is very different than that for the previously identified members; these new objects are distinctly fainter and redder on the whole than the previously identified sample. Objects with [4.5] > 11 are given the "faint" flag in Table 5.

About one-third of the new candidate YSO sample selected here are also retrieved from the $24 / 70$ space above and about two-thirds are also retrieved from the $K_{\mathrm{s}} / 24$ and 8/24 spaces above.

About $65 \%$ of these 65 candidate Taurus objects have Palomar and/or Keck spectroscopy (see Section 2.3), and nearly all are stellar; just four are dropped as galaxies.

\subsubsection{Selection via IRAC Color-color Diagram}

As our final selection mechanism, we use the IRAC CCD (as seen in, e.g., Allen et al. 2004). This parameter space, on its own, provides an initial sample size comparable to the previous $4.5 / 8$ color selection, as $13 \%$ of the entire catalog is detected in all four IRAC bands (to be compared with just $2 \%$ of the entire catalog being detected at MIPS-24, and $17 \%$ detected at 4.5 and $8 \mu \mathrm{m}$; see P10 for additional similar catalog statistics). However, by using the IRAC CCD on its own, we are blind to any luminosity information about the sources. This information was present previously because we were using CMDs, not CCDs. Given the surface density of galaxies, as well as the fact that the galaxy/YSO separation is not as vivid in this parameter space, the luminosity information is crucial. We imposed a requirement of [3.6] < 13.5, and the cut we used on the IRAC color-color space was (based on the literature discussions) [3.6]-[4.5] > 0.15 and [5.8]-[8] $>0.3$. This approach is different than in our consideration of the above parameter spaces, where we specifically called out YSO candidates fainter than a specific level.

Using these criteria, $\sim 265$ objects are selected, $\sim 100$ of which are previously identified Taurus members, $\sim 100$ of which are non-members (previously identified or new), and $\sim 65$ of which are new candidate YSOs. Of those, $\sim 35 \%$ were also 
found using $24 / 70, \sim 60 \%$ were found using $K_{\mathrm{s}} / 24$ or $8 / 24$, and $\sim 80 \%$ were found using $4.5 / 8$. (More on objects selected using all CMDs in Section 4.1 below.)

As can be seen in Figure 5, the previously identified Taurus members roughly fall into two groups: those with little or no IRAC excess, and those with substantial excesses. Among our new candidate members, we have some objects with little or no IRAC excess (selected from other parameter spaces), as well as objects with more substantial excesses, but the division is not as clean, suggesting contaminants in our YSO candidate list. We have also selected some objects that are very red in [5.8]-[8] but nearly colorless in [3.6]-[4.5]. These could be disks with large inner holes, or galaxies.

A little more than half of these 57 candidate objects have spectroscopy (see Section 2.3) from Palomar and/or Keck; just four are galaxies.

\subsection{Tables of Objects}

Now that we have used the Spitzer properties of the previously identified Taurus member sample to select a new candidate YSO sample, we present the data tables with observed and derived properties of both sets of objects. In this section, we review the contents of Tables 4-8. The contents of Tables 4 and 5 are similar but not identical, as are the contents of Tables 6 and 7 . Table 8 summarizes the new members, sorted by confidence level.

Tables 4 and 5 present, for each of the previously identified Taurus members and the new candidate Taurus members identified here, respectively, SST Tau names (note, as IAU-compliant names, the right ascension (R.A.) and declination (decl.) as given in the name are truncated, not rounded), a common name from previous studies (if applicable), and measurements in the Spitzer bands. For most of these objects, this is the first time that MIPS flux densities have appeared in the literature. Since many of the previously identified and new candidate Taurus members appear as having YSO-like colors in more than one of the CMD and CCD of Table 1 and Figures 1-5, Tables 4 and 5 also present the color criteria that are met by each object individually, for each of the color spaces we use here to identify YSO candidates. Objects appearing faint and red (see Section 3.1.4 above) are indicated as such. SEDs for each of the previously identified members and new candidates appear in Appendix A; the SED properties of the sample as a whole will be discussed in Section 4.5 below. Table 5 additionally contains notes about individual objects. These tables are sorted by SST Tau name, effectively sorted by R.A. and then decl.

Additional information about each of the previously identified Taurus members and the new candidate Taurus members identified here appears in Tables 6 and 7, respectively. Both of these tables start by repeating the SST Tau name, and a previous common name (if applicable). Table 7 then lists the grade ranking (see Section 3.1.4 above) that we assigned each of the candidate objects. Note that these tables are still sorted by position; Table 8 later presents the candidate Taurus members in order of confidence.

We compare our search method to others in the literature in Section 4.7 below; in preparation for that, Tables 6 and 7 indicate, for each of the previously identified Taurus members, how (or if) the c2d (Harvey et al. 2007) or Gutermuth et al. (2008) criteria for YSOs identified the object. (Note: whether or not our search recovered each previously identified object can be found in Table 4.)
Tables 6 and 7 contain a YSO classification. The near- to mid-IR slope of each SED, $\alpha$, is what we used for determining a YSO classification for these objects. For each of the previously identified and new candidate objects in our survey, we performed a simple ordinary least-squares linear fit to all available photometry (just detections, not including upper or lower limits) between 2 and $24 \mu \mathrm{m}$, inclusive. Note that errors on all of the IR points are so small as to not affect the fitted SED slope, and that a forthcoming paper will investigate the (small) effects of fitting a line to all available points within a different wavelength range, e.g., 3.6-24 $\mu \mathrm{m}$. In the spirit of Wilking et al. (2001), we define $\alpha=d \log \lambda F_{\lambda} / d \log \lambda$, where $\alpha>0.3$ for a Class I, 0.3 to -0.3 for a flat-spectrum source, -0.3 to -1.6 for a Class II, and $<-1.6$ for a Class III. We realize that the precise definition of $\alpha$ can vary, resulting in different classifications for certain objects; detailed discussion of this issue is beyond the scope of this paper. Classification via this method is provided for all previously identified and new candidate objects specifically to enable comparison within this paper via internally consistent means.

Adopted spectral types appear in Tables 6 and 7. This spectral type comes from the literature (see Section 3.1.1) or from our spectra (Section 2.3); if the latter, it is indicated as such in the "notes" column. If we obtained a spectrum for the object, and if $\mathrm{H} \alpha$ was measurable, we report the equivalent width in these tables (see Section 2.3 for analysis details). If the Ca IR triplet was in emission at the time of our observation, it is noted in the "notes" column.

Tables 6 and 7 include an estimate of the star's luminosity $\left(L_{*}\right)$ and an estimate of the ratio of the IR excess luminosity to the star's total (photospheric $+\mathrm{IR})$ luminosity $\left(L_{\mathrm{IR}} / L_{\text {total }}\right.$, where $\left.L_{\text {total }}=L_{*}+L_{\mathrm{IR}}\right)$. We describe briefly our procedure to determine the IR luminosity. Many faint sources were undetected at the longest wavelengths; we extrapolated missing data points for wavelengths longer than $8.0 \mu \mathrm{m}$. We used the longest wavelength available data point as a reference and assumed that its flux density corresponded to blackbody emission peaking at that wavelength. We then used this blackbody function to estimate the missing fluxes at the longer wavelengths. We compared the measured $J-H$ colors and the expected photospheric $(J-H)_{0}$ colors (as tabulated in Chapter 7 of Allen's Astrophysical Quantities; Cox 2000), attributing any difference to extinction, $A_{V}$, and we corrected the photometric fluxes for it. We obtained a spline curve through the corrected fluxes in $\log F_{\lambda}, \log \lambda$ space on a wavelength grid from $0.1 \AA$ to $1000 \AA$ with a $\Delta \lambda=0.02 \AA$. To determine the IR excess, we determined the underlying stellar photospheric emission using PHOENIX stellar atmosphere models for $T_{\text {eff }} \leqslant 10,000 \mathrm{~K}$ and Kurucz models for $T_{\text {eff }}>10,000 \mathrm{~K}$ and matched the stellar atmosphere model to the corrected $J$-band fluxes. The IR luminosity was calculated by measuring the difference between the spline curve and the stellar atmosphere model. We emphasize that care was taken to ensure that we included only adequate excess luminosity contributions at each wavelength of the grid (e.g., if the stellar atmosphere model showed a drop in flux between the $K_{\mathrm{s}}$ and $3.6 \mu \mathrm{m}$ fluxes, and the spline curve was above but there was no obvious sign of IR excess at those wavelengths, the contribution to the IR luminosity was not included). We also only included contributions if the spline curve was at least 1.5 times larger than the stellar atmosphere contribution. Finally, we determined if the calculated IR luminosity was an upper limit by checking that at least one of the corrected photometric fluxes beyond $3.6 \mu \mathrm{m}$ were real and not extrapolated. If the latter, the IR luminosity 
Table 4

Spitzer Measurements for Sample of Previously Identified Taurus Members ${ }^{\mathrm{a}}$

\begin{tabular}{|c|c|c|c|c|c|c|c|c|c|c|c|c|c|c|}
\hline SST Tau Name & Common Name & $\begin{array}{l}{[3.6]} \\
\text { (mag) }\end{array}$ & $\begin{array}{l}{[4.5]} \\
(\mathrm{mag})\end{array}$ & $\begin{array}{l}{[5.8]} \\
(\mathrm{mag})\end{array}$ & $\begin{array}{c}{[8]} \\
(\mathrm{mag})\end{array}$ & $\begin{array}{c}{[24]} \\
(\mathrm{mag})\end{array}$ & $\begin{array}{c}{[70]} \\
(\mathrm{mag})\end{array}$ & $\begin{array}{l}{[160]} \\
(\mathrm{mag})\end{array}$ & $\begin{array}{l}\text { Ident. in } \\
24 / 70 \\
\text { CMD }\end{array}$ & $\begin{array}{c}\text { Ident. in } \\
K_{\mathrm{S}} / 24 \\
\mathrm{CMD}\end{array}$ & $\begin{array}{c}\text { Ident. in } \\
8 / 24 \\
\text { CMD }\end{array}$ & $\begin{array}{c}\text { Ident. in } \\
4.5 / 8 \\
\text { CMD }\end{array}$ & $\begin{array}{l}\text { Ident. in } \\
\text { IRAC } \\
\text { CCD }\end{array}$ & Notes \\
\hline $041314.1+281910$ & LkCa 1 & $8.54 \pm 0.05$ & $8.50 \pm 0.05$ & $8.41 \pm 0.05$ & $8.43 \pm 0.05$ & $8.28 \pm 0.08$ & $>1.30$ & & & No & No & No & No & \\
\hline $041327.2+281624$ & Anon 1 & $7.23 \pm 0.05$ & $7.24 \pm 0.05$ & $7.17 \pm 0.05$ & $7.08 \pm 0.05$ & $6.95 \pm 0.05$ & $>1.27$ & $\ldots$ & $\ldots$ & No & No & No & No & \\
\hline $041353.2+281123$ & IRAS04108+2803 A & $9.02 \pm 0.05$ & $8.37 \pm 0.05$ & $7.67 \pm 0.05$ & $6.57 \pm 0.05$ & $3.44 \pm 0.04$ & $>0.19$ & $>-2.05$ & $\ldots$ & Yes & Yes & Yes & Yes & $\mathrm{b}$ \\
\hline $041354.7+281132$ & IRAS04108+2803 B & $9.38 \pm 0.05$ & $8.03 \pm 0.05$ & $6.96 \pm 0.05$ & $5.78 \pm 0.05$ & $1.38 \pm 0.04$ & $-1.84 \pm 0.22$ & $>-1.94$ & Yes & Yes & Yes & Yes & Yes & $\mathrm{b}$ \\
\hline $041357.3+291819$ & IRAS04108+2910 & $7.48 \pm 0.05$ & $6.84 \pm 0.05$ & $6.26 \pm 0.05$ & $5.54 \pm 0.05$ & $3.13 \pm 0.04$ & $1.15 \pm 0.22$ & $>-3.39$ & Yes & Yes & Yes & Yes & Yes & $\ldots$ \\
\hline $041411.8+281153$ & $\mathrm{~J} 04141188+2811535$ & $10.93 \pm 0.06$ & $10.40 \pm 0.05$ & $10.12 \pm 0.07$ & $8.99 \pm 0.06$ & $5.76 \pm 0.01$ & $>1.03$ & $\ldots$ & & Yes & Yes & Yes & Yes & $\ldots$ \\
\hline $041412.2+280837$ & IRAS04111+2800G & $13.19 \pm 0.06$ & $11.90 \pm 0.06$ & $11.19 \pm 0.06$ & $10.39 \pm 0.06$ & $3.47 \pm 0.04$ & $-0.33 \pm 0.22$ & $\ldots$ & Yes & $\ldots$ & Yes-faint & Yes-faint & Yes & $\ldots$ \\
\hline $041412.9+281212$ & V773 Tau ABC & $<6.62$ & $<6.10$ & $5.13 \pm 0.05$ & $4.38 \pm 0.05$ & $1.69 \pm 0.04$ & $0.27 \pm 0.22$ & $\ldots$ & Yes & Yes & Yes & $\ldots$ & & $\ldots$ \\
\hline $041413.5+281249$ & FM Tau & $8.09 \pm 0.05$ & $7.67 \pm 0.05$ & $7.36 \pm 0.05$ & $6.42 \pm 0.05$ & $2.92 \pm 0.04$ & $1.07 \pm 0.22$ & $\ldots$ & Yes & Yes & Yes & Yes & Yes & $\ldots$ \\
\hline $041414.5+282758$ & FN Tau & $7.59 \pm 0.05$ & $7.17 \pm 0.05$ & $6.71 \pm 0.05$ & $5.75 \pm 0.05$ & $2.03 \pm 0.04$ & $-0.25 \pm 0.22$ & $\ldots$ & Yes & Yes & Yes & Yes & Yes & $\ldots$ \\
\hline $041417.0+281057$ & CW Tau & $<6.62$ & $<6.10$ & $5.08 \pm 0.05$ & $4.51 \pm 0.05$ & $1.75 \pm 0.04$ & $-0.42 \pm 0.22$ & $\ldots$ & Yes & Yes & Yes & . & . & $\ldots$ \\
\hline $041417.6+280609$ & CIDA-1 & $8.67 \pm 0.05$ & $8.13 \pm 0.05$ & $7.59 \pm 0.05$ & $6.71 \pm 0.05$ & $3.53 \pm 0.04$ & $1.28 \pm 0.22$ & . & Yes & Yes & Yes & Yes & Yes & $\ldots$ \\
\hline $041426.2+280603$ & IRAS04113+2758 A & $<6.62$ & $<6.10$ & $4.63 \pm 0.05$ & $3.79 \pm 0.05$ & $<0.45$ & $-2.54 \pm 0.22$ & $-4.35 \pm 0.34$ & $\ldots$ & $\ldots$ & $\ldots$ & & & $\mathrm{f}$ \\
\hline $041430.5+280514$ & MHO-3 & $7.22 \pm 0.05$ & $6.49 \pm 0.05$ & $5.75 \pm 0.05$ & $4.53 \pm 0.05$ & $<0.45$ & $-1.06 \pm 0.22$ & $-4.15 \pm 0.34$ & $\ldots$ & $\ldots$ & $\ldots$ & Yes & Yes & $\ldots$ \\
\hline $041447.3+264626$ & FP Tau & $8.11 \pm 0.05$ & $7.86 \pm 0.05$ & $7.60 \pm 0.05$ & $7.27 \pm 0.05$ & $4.25 \pm 0.04$ & $1.22 \pm 0.22$ & $\ldots$ & Yes & Yes & Yes & Yes & Yes & $\ldots$ \\
\hline $041447.8+264811$ & CX Tau & $8.48 \pm 0.05$ & $8.13 \pm 0.05$ & $7.68 \pm 0.05$ & $6.63 \pm 0.05$ & $3.35 \pm 0.04$ & $1.23 \pm 0.22$ & $\ldots$ & Yes & Yes & Yes & Yes & Yes & $\ldots$ \\
\hline $041447.9+275234$ & $\mathrm{LkCa} 3 \mathrm{AB}$ & $7.28 \pm 0.05$ & $7.33 \pm 0.05$ & $7.27 \pm 0.05$ & $7.23 \pm 0.05$ & $7.07 \pm 0.05$ & $>1.17$ & $\ldots$ & $\ldots$ & No & No & No & No & $\ldots$ \\
\hline $041449.2+281230$ & FO Tau AB & $7.53 \pm 0.05$ & $7.17 \pm 0.05$ & $6.72 \pm 0.05$ & $5.92 \pm 0.05$ & $2.83 \pm 0.04$ & $0.79 \pm 0.22$ & $\ldots$ & Yes & Yes & Yes & Yes & Yes & $\ldots$ \\
\hline $041505.1+280846$ & CIDA-2 & $8.90 \pm 0.05$ & $8.79 \pm 0.05$ & $8.71 \pm 0.05$ & $8.68 \pm 0.05$ & $8.45 \pm 0.11$ & $>1.31$ & $\ldots$ & $\ldots$ & No & No & No & No & $\ldots$ \\
\hline $041514.7+280009$ & KPNO-1 & $13.23 \pm 0.13$ & $12.72 \pm 0.22$ & $12.94 \pm 0.09$ & $12.81 \pm 0.10$ & $>10.61$ & $>0.90$ & $\ldots$ & $\ldots$ & $\ldots$ & $\ldots$ & No & No & $\ldots$ \\
\hline $041524.0+291043$ & J04152409+2910434 & $11.86 \pm 0.05$ & $11.79 \pm 0.05$ & $11.66 \pm 0.06$ & $11.48 \pm 0.06$ & $>10.06$ & $>1.14$ & $\ldots$ & $\ldots$ & $\ldots$ & $\ldots$ & No & No & $\ldots$ \\
\hline $041612.1+275638$ & J04161210+2756385 & $9.38 \pm 0.05$ & $9.04 \pm 0.05$ & $8.71 \pm 0.05$ & $8.30 \pm 0.05$ & $5.37 \pm 0.04$ & $1.55 \pm 0.22$ & $\ldots$ & Yes & Yes & Yes & Yes & Yes & $\ldots$ \\
\hline $041618.8+275215$ & J04161885+2752155 & $10.88 \pm 0.05$ & $10.78 \pm 0.05$ & $10.67 \pm 0.06$ & $10.68 \pm 0.06$ & $>9.95$ & $>1.26$ & $\ldots$ & $\ldots$ & $\ldots$ & $\ldots$ & No & No & $\ldots$ \\
\hline $041628.1+280735$ & $\mathrm{LkCa} 4$ & $8.18 \pm 0.05$ & $8.17 \pm 0.05$ & $8.04 \pm 0.05$ & $8.05 \pm 0.05$ & $7.94 \pm 0.07$ & $>1.20$ & . & & No & No & No & No & $\ldots$ \\
\hline $041639.1+285849$ & J04163911+2858491 & $10.50 \pm 0.05$ & $10.14 \pm 0.05$ & $9.86 \pm 0.05$ & $9.41 \pm 0.05$ & $7.22 \pm 0.05$ & $>1.24$ & & & Yes & Yes & Yes & Yes & $\ldots$ \\
\hline $041733.7+282046$ & CY Tau & $7.87 \pm 0.05$ & $7.53 \pm 0.05$ & $7.27 \pm 0.05$ & $6.72 \pm 0.05$ & $4.42 \pm 0.04$ & $1.87 \pm 0.22$ & $>-1.41$ & Yes & Yes & Yes & Yes & Yes & $\ldots$ \\
\hline $041738.9+283300$ & LkCa 5 & $8.93 \pm 0.05$ & $\ldots$ & $8.80 \pm 0.05$ & $\ldots$ & $8.66 \pm 0.09$ & $>1.61$ & $\ldots$ & $\ldots$ & No & $\ldots$ & $\ldots$ & $\ldots$ & $\ldots$ \\
\hline $041749.5+281331$ & KPNO-10 & $10.82 \pm 0.05$ & $10.36 \pm 0.05$ & $9.81 \pm 0.05$ & $8.89 \pm 0.05$ & $5.96 \pm 0.04$ & $1.92 \pm 0.22$ & $\ldots$ & Yes & Yes & Yes & Yes & Yes & $\ldots$ \\
\hline $041749.6+282936$ & V410 X-ray 1 & $8.41 \pm 0.05$ & $7.85 \pm 0.05$ & $7.42 \pm 0.05$ & $6.47 \pm 0.05$ & $3.78 \pm 0.04$ & $2.95 \pm 0.22$ & $\ldots$ & Yes & Yes & Yes & Yes & Yes & $\ldots$ \\
\hline $041807.9+282603$ & V410 X-ray 3 & $10.04 \pm 0.05$ & $9.94 \pm 0.05$ & $9.90 \pm 0.06$ & $9.80 \pm 0.05$ & $9.27 \pm 0.21$ & $>1.79$ & .. & $\ldots$ & Yes & Yes-faint & No & No & $\ldots$ \\
\hline $041817.1+282841$ & V410 Anon 13 & $10.23 \pm 0.05$ & $9.94 \pm 0.05$ & $9.49 \pm 0.05$ & $8.81 \pm 0.05$ & $6.04 \pm 0.04$ & & $>-0.57$ & $\ldots$ & Yes & Yes & Yes & Yes & $\ldots$ \\
\hline $041822.3+282437$ & V410 Anon 24 & $9.84 \pm 0.05$ & $9.54 \pm 0.05$ & $9.34 \pm 0.05$ & $9.38 \pm 0.05$ & $9.29 \pm 0.10$ & $>1.60$ & $>1.50$ & $\ldots$ & Yes & No & No & No & $\ldots$ \\
\hline $041829.0+282619$ & V410 Anon 25 & $8.87 \pm 0.05$ & $8.64 \pm 0.05$ & $8.46 \pm 0.05$ & $8.39 \pm 0.05$ & $8.15 \pm 0.08$ & $>1.67$ & $>0.12$ & $\ldots$ & Yes & No & No & No & $\ldots$ \\
\hline $041830.3+274320$ & KPNO-11 & $10.71 \pm 0.05$ & $10.59 \pm 0.05$ & $10.60 \pm 0.06$ & $10.50 \pm 0.06$ & $>10.01$ & $>1.46$ & $\ldots$ & $\ldots$ & $\ldots$ & $\ldots$ & No & No & $\ldots$ \\
\hline $041831.1+282716$ & V410 Tau ABC & $7.36 \pm 0.05$ & $7.34 \pm 0.05$ & $7.34 \pm 0.05$ & $7.25 \pm 0.05$ & $7.14 \pm 0.06$ & $>1.65$ & $\ldots$ & $\ldots$ & No & No & No & No & $\ldots$ \\
\hline $041831.1+281629$ & DD Tau AB & $<6.62$ & $<6.10$ & $5.29 \pm 0.05$ & $4.48 \pm 0.05$ & $1.75 \pm 0.04$ & $-0.04 \pm 0.22$ & $\ldots$ & Yes & Yes & Yes & & & $\ldots$ \\
\hline $041831.5+281658$ & CZ Tau AB & $8.46 \pm 0.05$ & $7.63 \pm 0.05$ & $6.62 \pm 0.05$ & $5.00 \pm 0.05$ & $1.96 \pm 0.04$ & $1.45 \pm 0.22$ & $\ldots$ & Yes & Yes & Yes & Yes & Yes & $\ldots$ \\
\hline $041832.0+283115$ & IRAS04154+2823 & $7.57 \pm 0.05$ & $7.07 \pm 0.05$ & $6.12 \pm 0.05$ & $5.52 \pm 0.05$ & $1.91 \pm 0.04$ & $-0.38 \pm 0.22$ & $\ldots$ & Yes & Yes & Yes & Yes & Yes & $\ldots$ \\
\hline $041834.4+283030$ & V410 X-ray 2 & $8.35 \pm 0.05$ & $8.09 \pm 0.05$ & $7.80 \pm 0.05$ & $7.55 \pm 0.05$ & $3.41 \pm 0.04$ & $0.31 \pm 0.22$ & $\ldots$ & Yes & Yes & Yes & Yes & No & $\ldots$ \\
\hline $041840.2+282424$ & V410 X-ray 4 & $8.91 \pm 0.05$ & $8.64 \pm 0.05$ & $8.44 \pm 0.05$ & $8.43 \pm 0.05$ & $8.09 \pm 0.08$ & $>1.60$ & $>-2.59$ & $\ldots$ & Yes & No & No & No & $\ldots$ \\
\hline $041840.6+281915$ & V892 Tau & $<6.62$ & $<6.10$ & $3.61 \pm 0.05$ & $<3.52$ & $<0.45$ & $<-2.30$ & $<-4.90$ & $\ldots$ & $\ldots$ & $\ldots$ & $\ldots$ & $\ldots$ & $\mathrm{cd}$ \\
\hline $041841.3+282725$ & LR1 & $9.50 \pm 0.05$ & $8.92 \pm 0.05$ & $8.44 \pm 0.05$ & $7.95 \pm 0.05$ & $4.65 \pm 0.04$ & $0.38 \pm 0.22$ & $>-0.29$ & Yes & Yes & Yes & Yes & Yes & $\ldots$ \\
\hline $041842.5+281849$ & V410 X-ray 7 & $8.73 \pm 0.05$ & $8.61 \pm 0.05$ & $8.35 \pm 0.05$ & $8.09 \pm 0.07$ & $5.15 \pm 0.01$ & $>-0.30$ & $>-2.88$ & $\ldots$ & Yes & Yes & Yes & No & $\ldots$ \\
\hline $041845.0+282052$ & V410 Anon 20 & $11.01 \pm 0.05$ & $10.74 \pm 0.05$ & $10.55 \pm 0.06$ & $10.57 \pm 0.06$ & $>10.20$ & $>0.49$ & $>-3.20$ & $\ldots$ & $\ldots$ & $\ldots$ & No & No & $\ldots$ \\
\hline $041847.0+282007$ & Hubble 4 & $7.09 \pm 0.05$ & $7.04 \pm 0.05$ & $6.95 \pm 0.05$ & $6.96 \pm 0.05$ & $6.78 \pm 0.01$ & $>0.18$ & $>-4.19$ & $\ldots$ & No & No & No & No & . \\
\hline
\end{tabular}




\begin{tabular}{|c|c|c|c|c|c|c|c|c|c|c|c|c|c|c|}
\hline SST Tau Name & Common Name & $\begin{array}{l}{[3.6]} \\
(\mathrm{mag})\end{array}$ & $\begin{array}{c}4.5] \\
(\mathrm{mag})\end{array}$ & $\begin{array}{l}5.8] \\
(\mathrm{mag})\end{array}$ & $\begin{array}{c}8] \\
(\mathrm{mag})\end{array}$ & $\begin{array}{c}{[24]} \\
(\mathrm{mag})\end{array}$ & $\begin{array}{c}70] \\
(\mathrm{mag})\end{array}$ & $\begin{array}{l}{[160]} \\
(\mathrm{mag})\end{array}$ & $\begin{array}{c}\text { Ident. in } \\
24 / 70 \\
\text { CMD }\end{array}$ & $\begin{array}{c}\text { Ident. in } \\
K_{\mathrm{S}} / 24 \\
\mathrm{CMD}\end{array}$ & $\begin{array}{c}\text { Ident. in } \\
8 / 24 \\
\text { CMD } \\
\end{array}$ & $\begin{array}{c}\text { Ident. in } \\
4.5 / 8 \\
\text { CMD }\end{array}$ & $\begin{array}{c}\text { Ident. in } \\
\text { IRAC } \\
\text { CCD }\end{array}$ & Notes \\
\hline $041851.1+281433$ & KPNO-2 & $12.25 \pm 0.05$ & $12.11 \pm 0.06$ & $12.02 \pm 0.06$ & $11.84 \pm 0.07$ & $>9.59$ & $>1.59$ & & & & & No & No & $\ldots$ \\
\hline $041851.4+282026$ & CoKu Tau/1 & $10.22 \pm 0.05$ & $9.02 \pm 0.05$ & $7.72 \pm 0.05$ & $5.87 \pm 0.05$ & $1.07 \pm 0.04$ & $-0.98 \pm 0.22$ & $<-2.55$ & Yes & Yes & Yes & Yes & Yes & $\mathrm{c}$ \\
\hline $041858.1+281223$ & IRAS04158+2805 & $9.23 \pm 0.05$ & $8.54 \pm 0.05$ & $7.85 \pm 0.06$ & $6.84 \pm 0.05$ & $2.73 \pm 0.04$ & $-0.07 \pm 0.22$ & $-2.51 \pm 0.22$ & Yes & Yes & Yes & Yes & Yes & $\ldots$ \\
\hline $041901.1+281942$ & V410 X-ray 6 & $8.76 \pm 0.05$ & $8.67 \pm 0.05$ & $8.54 \pm 0.05$ & $8.26 \pm 0.05$ & $3.82 \pm 0.04$ & $0.69 \pm 0.22$ & & Yes & Yes & Yes & Yes & No & $\ldots$ \\
\hline $041901.2+280248$ & KPNO-12 & $13.97 \pm 0.06$ & $13.61 \pm 0.06$ & $13.23 \pm 0.08$ & $12.75 \pm 0.08$ & $>10.13$ & $>1.74$ & $>-0.54$ & $\ldots$ & $\ldots$ & $\ldots$ & No & No & $\ldots$ \\
\hline $041901.9+282233$ & V410 Tau X-ray 5a & $9.64 \pm 0.05$ & $9.55 \pm 0.05$ & $9.43 \pm 0.05$ & $9.39 \pm 0.05$ & $8.88 \pm 0.12$ & $>1.59$ & $>-1.28$ & $\ldots$ & Yes & Yes & No & No & $\ldots$ \\
\hline $041912.8+282933$ & FQ Tau AB & $8.78 \pm 0.05$ & $8.42 \pm 0.05$ & $8.12 \pm 0.05$ & $7.41 \pm 0.05$ & $4.85 \pm 0.04$ & $2.16 \pm 0.22$ & $\ldots$ & Yes & Yes & Yes & Yes & Yes & $\ldots$ \\
\hline $041915.8+290626$ & BP Tau & $7.27 \pm 0.05$ & $6.90 \pm 0.05$ & $6.65 \pm 0.05$ & $5.71 \pm 0.05$ & $2.52 \pm 0.04$ & $0.71 \pm 0.22$ & $\ldots$ & Yes & Yes & Yes & Yes & Yes & $\ldots$ \\
\hline $041926.2+282614$ & V819 Tau & $8.20 \pm 0.05$ & $8.29 \pm 0.05$ & $8.11 \pm 0.05$ & $8.06 \pm 0.05$ & $6.29 \pm 0.05$ & & $\ldots$ & & Yes & Yes & No & No & $\ldots$ \\
\hline $041935.4+282721$ & FR Tau & $9.42 \pm 0.05$ & $8.93 \pm 0.05$ & $8.25 \pm 0.05$ & $7.27 \pm 0.05$ & $4.84 \pm 0.04$ & $3.14 \pm 0.22$ & $\ldots$ & Yes & Yes & Yes & Yes & Yes & $\ldots$ \\
\hline $041941.2+274948$ & $\mathrm{LkCa} 7 \mathrm{AB}$ & $8.11 \pm 0.05$ & $8.11 \pm 0.05$ & $8.04 \pm 0.05$ & $7.99 \pm 0.05$ & $7.75 \pm 0.06$ & $>1.94$ & $\ldots$ & $\ldots$ & No & No & No & No & $\ldots$ \\
\hline $041942.5+271336$ & IRAS04166+2706 & $12.84 \pm 0.06$ & $11.32 \pm 0.05$ & $10.49 \pm 0.06$ & $9.75 \pm 0.06$ & $2.93 \pm 0.04$ & $-1.92 \pm 0.22$ & $-4.53 \pm 0.34$ & Yes & $\ldots$ & Yes-faint & Yes-faint & Yes & $\ldots$ \\
\hline $041958.4+270957$ & IRAS04169+2702 & $8.41 \pm 0.05$ & $7.15 \pm 0.05$ & $6.29 \pm 0.05$ & $5.33 \pm 0.05$ & $0.66 \pm 0.04$ & $<-2.30$ & $-5.40 \pm 0.34$ & & Yes & Yes & Yes & Yes & $\ldots$ \\
\hline $042025.5+270035$ & $\mathrm{~J} 04202555+2700355$ & $10.99 \pm 0.05$ & $10.77 \pm 0.05$ & $10.44 \pm 0.06$ & $9.74 \pm 0.05$ & $6.13 \pm 0.04$ & $2.49 \pm 0.22$ & $\ldots$ & Yes & Yes & Yes-faint & Yes & Yes & $\ldots$ \\
\hline $042039.1+271731$ & 2MASS J04203918+2717317 & $9.42 \pm 0.05$ & $9.39 \pm 0.05$ & $9.35 \pm 0.05$ & $9.29 \pm 0.05$ & $8.83 \pm 0.10$ & $>1.50$ & $\ldots$ & $\ldots$ & No & No & No & No & $\ldots$ \\
\hline $042107.9+270220$ & CFHT-19 & $7.54 \pm 0.05$ & $6.66 \pm 0.05$ & $6.01 \pm 0.05$ & $5.10 \pm 0.05$ & $1.61 \pm 0.04$ & $-1.18 \pm 0.22$ & $<-3.27$ & Yes & Yes & Yes & Yes & Yes & $\mathrm{c}$ \\
\hline $042110.3+270137$ & IRAS04181+2654B & $9.03 \pm 0.05$ & $8.24 \pm 0.05$ & $7.60 \pm 0.05$ & $6.70 \pm 0.05$ & $2.69 \pm 0.04$ & $-0.47 \pm 0.22$ & $<-3.97$ & Yes & Yes & Yes & Yes & Yes & $\mathrm{bc}$ \\
\hline $042111.4+270109$ & IRAS04181+2654A & $8.60 \pm 0.05$ & $7.56 \pm 0.05$ & $6.71 \pm 0.05$ & $5.71 \pm 0.05$ & $1.64 \pm 0.04$ & $-1.04 \pm 0.22$ & $-4.21 \pm 0.34$ & Yes & Yes & Yes & Yes & Yes & $\mathrm{b}$ \\
\hline $042134.5+270138$ & $\mathrm{~J} 04213459+2701388$ & $9.86 \pm 0.05$ & $9.65 \pm 0.05$ & $9.35 \pm 0.05$ & $8.98 \pm 0.05$ & $7.18 \pm 0.05$ & $>1.64$ & $\ldots$ & $\ldots$ & Yes & Yes & Yes & Yes & $\ldots$ \\
\hline $042146.3+265929$ & CFHT-10 & $11.54 \pm 0.05$ & $11.32 \pm 0.05$ & $11.05 \pm 0.06$ & $10.45 \pm 0.06$ & $7.26 \pm 0.05$ & $>1.45$ & $\ldots$ & $\ldots$ & Yes & No & Yes-faint & Yes & $\ldots$ \\
\hline $042154.5+265231$ & $\mathrm{~J} 04215450+2652315$ & $13.22 \pm 0.06$ & $13.12 \pm 0.06$ & $12.90 \pm 0.07$ & $12.80 \pm 0.08$ & $10.50 \pm 0.22$ & $>1.66$ & $\ldots$ & $\ldots$ & Yes-faint & No & No & No & $\ldots$ \\
\hline $042155.6+275506$ & DE Tau & $7.07 \pm 0.05$ & $6.73 \pm 0.05$ & $6.40 \pm 0.05$ & $5.78 \pm 0.05$ & $2.58 \pm 0.04$ & $-0.19 \pm 0.22$ & $\ldots$ & Yes & Yes & Yes & Yes & Yes & $\ldots$ \\
\hline $042157.4+282635$ & RY Tau & $<6.62$ & $<6.10$ & $3.60 \pm 0.05$ & $<3.52$ & $<0.45$ & $<-2.30$ & $-4.24 \pm 0.34$ & $\ldots$ & $\ldots$ & $\ldots$ & $\ldots$ & $\ldots$ & $\ldots$ \\
\hline $042158.8+281806$ & HD283572 & $86 \pm 0.05$ & $6.86 \pm 0.05$ & $6.81 \pm 0.05$ & $6.78 \pm 0.05$ & $6.76 \pm 0.05$ & $>1.24$ & $\ldots$ & $\ldots$ & No & No & No & No & $\ldots$ \\
\hline $042200.6+265732$ & FS Tau B & $9.66 \pm 0.05$ & $8.40 \pm 0.05$ & $7.23 \pm 0.05$ & $5.95 \pm 0.05$ & $1.58 \pm 0.04$ & $-0.68 \pm 0.22$ & $<-4.14$ & Yes & Yes & Yes & Yes & Yes & $\mathrm{bc}$ \\
\hline $042202.1+265730$ & FS Tau Aab & $6.75 \pm 0.05$ & $6.30 \pm 0.05$ & $5.81 \pm 0.05$ & $4.99 \pm 0.05$ & $1.33 \pm 0.04$ & $>0.05$ & $\ldots$ & & Yes & Yes & Yes & Yes & $\ldots$ \\
\hline $042203.1+282538$ & LkCa 21 & $8.26 \pm 0.05$ & $8.22 \pm 0.05$ & $8.14 \pm 0.05$ & $8.06 \pm 0.05$ & $8.06 \pm 0.09$ & $>1.23$ & $\ldots$ & & No & No & No & No & $\ldots$ \\
\hline $042216.4+254911$ & CFHT-14 & $11.48 \pm 0.05$ & $11.34 \pm 0.05$ & $11.28 \pm 0.06$ & $11.23 \pm 0.06$ & $>9.51$ & $>1.16$ & $\ldots$ & $\ldots$ & $\ldots$ & $\ldots$ & No & No & $\ldots$ \\
\hline $042216.7+265457$ & CFHT-21 & $7.77 \pm 0.05$ & $7.26 \pm 0.05$ & $6.85 \pm 0.05$ & $6.30 \pm 0.05$ & $3.29 \pm 0.04$ & $1.18 \pm 0.22$ & & Yes & Yes & Yes & Yes & Yes & $\ldots$ \\
\hline $042224.0+264625$ & 2MASS J04222404+2646258 & $9.52 \pm 0.05$ & $9.40 \pm 0.05$ & $9.34 \pm 0.05$ & $9.33 \pm 0.05$ & $9.07 \pm 0.12$ & $>1.56$ & $\ldots$ & $\ldots$ & No & No & No & No & $\ldots$ \\
\hline $042307.7+280557$ & IRAS04200+2759 & $8.43 \pm 0.05$ & $7.81 \pm 0.05$ & $7.28 \pm 0.05$ & $6.44 \pm 0.05$ & $3.23 \pm 0.04$ & $0.76 \pm 0.22$ & $\ldots$ & Yes & Yes & Yes & Yes & Yes & $\ldots$ \\
\hline $042339.1+245614$ & FT Tau & $7.93 \pm 0.05$ & $7.46 \pm 0.05$ & $7.19 \pm 0.05$ & $6.29 \pm 0.05$ & $3.15 \pm 0.04$ & $0.28 \pm 0.22$ & $\ldots$ & Yes & Yes & Yes & Yes & Yes & $\ldots$ \\
\hline $042426.4+264950$ & CFHT-9 & $11.16 \pm 0.05$ & $10.88 \pm 0.05$ & $10.51 \pm 0.06$ & $9.83 \pm 0.05$ & $6.78 \pm 0.05$ & $>0.77$ & $\ldots$ & $\ldots$ & Yes & Yes-faint & Yes & Yes & $\ldots$ \\
\hline $042444.5+261014$ & IRAS04216+2603 & $8.08 \pm 0.05$ & $7.57 \pm 0.05$ & $7.14 \pm 0.05$ & $6.32 \pm 0.05$ & $3.53 \pm 0.04$ & $0.16 \pm 0.22$ & $-2.47 \pm 0.22$ & Yes & Yes & Yes & Yes & Yes & $\ldots$ \\
\hline $042445.0+270144$ & $\mathrm{~J} 1-4423$ & $10.21 \pm 0.05$ & $10.15 \pm 0.05$ & $10.06 \pm 0.06$ & $10.11 \pm 0.06$ & $>9.49$ & $>1.05$ & $\ldots$ & $\ldots$ & $\ldots$ & $\ldots$ & No & No & $\ldots$ \\
\hline $042449.0+264310$ & RXJ0424.8 & $7.73 \pm 0.05$ & $7.70 \pm 0.05$ & $7.69 \pm 0.05$ & $7.65 \pm 0.05$ & $7.40 \pm 0.06$ & $>1.02$ & $\ldots$ & $\ldots$ & No & No & No & No & $\ldots$ \\
\hline $042457.0+271156$ & IP Tau & $7.77 \pm 0.05$ & $7.45 \pm 0.05$ & $7.24 \pm 0.05$ & $6.60 \pm 0.05$ & $3.48 \pm 0.04$ & $0.74 \pm 0.22$ & $\ldots$ & Yes & Yes & Yes & Yes & Yes & $\ldots$ \\
\hline $042517.6+261750$ & $\mathrm{~J} 1-4872 \mathrm{AB}$ & $8.21 \pm 0.05$ & $8.20 \pm 0.05$ & $8.08 \pm 0.05$ & $8.06 \pm 0.05$ & $7.77 \pm 0.07$ & $>1.10$ & $\ldots$ & $\ldots$ & No & No & No & No & $\ldots$ \\
\hline $042629.3+262413$ & KPNO-3 & $11.41 \pm 0.05$ & $10.99 \pm 0.05$ & $10.49 \pm 0.06$ & $9.72 \pm 0.05$ & $6.86 \pm 0.05$ & $>1.09$ & $\ldots$ & $\ldots$ & Yes & Yes-faint & Yes & Yes & $\ldots$ \\
\hline $042630.5+244355$ & J04263055+2443558 & $12.57 \pm 0.05$ & $12.21 \pm 0.06$ & $11.76 \pm 0.06$ & $11.08 \pm 0.06$ & $8.87 \pm 0.15$ & $>1.09$ & $\ldots$ & $\ldots$ & Yes & No & No & Yes & $\ldots$ \\
\hline $042653.5+260654$ & FV Tau AB & $<6.62$ & $<6.10$ & $5.23 \pm 0.05$ & $4.56 \pm 0.05$ & $1.54 \pm 0.04$ & $-0.45 \pm 0.22$ & $\ldots$ & Yes & Yes & Yes & $\ldots$ & $\ldots$ & $\ldots$ \\
\hline $042654.4+260651$ & FV Tau/c AB & $8.01 \pm 0.05$ & $7.58 \pm 0.05$ & $7.05 \pm 0.05$ & $6.29 \pm 0.05$ & $3.88 \pm 0.04$ & $>0.72$ & $>-1.64$ & 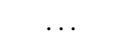 & Yes & Yes & Yes & Yes & $\ldots$ \\
\hline $042656.2+244335$ & IRAS04239+2436 & $7.61 \pm 0.05$ & $6.32 \pm 0.05$ & $5.38 \pm 0.05$ & $4.50 \pm 0.05$ & $<0.45$ & $-2.25 \pm 0.22$ & $-4.63 \pm 0.34$ & & $\ldots$ & $\ldots$ & Yes & Yes & $\ldots$ \\
\hline $042657.3+260628$ & KPNO-13 & $8.75 \pm 0.05$ & $8.33 \pm 0.05$ & $7.99 \pm 0.06$ & $7.35 \pm 0.05$ & $5.32 \pm 0.04$ & $>0.93$ & $>-2.25$ & & Yes & Yes & Yes & Yes & \\
\hline $042702.6+260530$ & DG Tau B & $8.77 \pm 0.05$ & . & $5.88 \pm 0.05$ & $5.24 \pm 0.05$ & $0.78 \pm 0.04$ & $-2.24 \pm 0.22$ & $-5.12 \pm 0.34$ & Yes & Yes & Yes & $\cdots$ & $\ldots$ & $\mathrm{b}$ \\
\hline
\end{tabular}




\begin{tabular}{|c|c|c|c|c|c|c|c|c|c|c|c|c|c|c|}
\hline SST Tau Name & Common Name & $\begin{array}{l}{[3.6]} \\
(\mathrm{mag})\end{array}$ & $\begin{array}{l}{[4.5]} \\
(\mathrm{mag})\end{array}$ & $\begin{array}{l}{[5.8]} \\
(\mathrm{mag})\end{array}$ & $\begin{array}{c}{[8]} \\
(\mathrm{mag})\end{array}$ & $\begin{array}{c}{[24]} \\
(\mathrm{mag})\end{array}$ & $\begin{array}{c}{[70]} \\
(\mathrm{mag})\end{array}$ & $\begin{array}{l}{[160]} \\
\text { (mag) }\end{array}$ & $\begin{array}{c}\text { Ident. in } \\
24 / 70 \\
\text { CMD }\end{array}$ & $\begin{array}{c}\text { Ident. in } \\
K_{\mathrm{s}} / 24 \\
\mathrm{CMD}\end{array}$ & $\begin{array}{c}\text { Ident. in } \\
8 / 24 \\
\text { CMD } \\
\end{array}$ & $\begin{array}{c}\text { Ident. in } \\
4.5 / 8 \\
\text { CMD }\end{array}$ & $\begin{array}{c}\text { Ident. in } \\
\text { IRAC } \\
\text { CCD }\end{array}$ & Notes \\
\hline $042702.8+254222$ & DF Tau AB & $<6.62$ & $<6.10$ & $5.08 \pm 0.05$ & $4.50 \pm 0.05$ & $2.19 \pm 0.04$ & $0.70 \pm 0.22$ & & Yes & Yes & Yes & $\cdots$ & $\cdots$ & \\
\hline $042704.6+260616$ & DG Tau A & $<6.62$ & $<6.10$ & $4.67 \pm 0.05$ & $3.55 \pm 0.05$ & $<0.45$ & $<-2.30$ & $<-4.46$ & $\ldots$ & & & & & $\mathrm{bc}$ \\
\hline $042727.9+261205$ & KPNO-4 & $12.57 \pm 0.05$ & $12.37 \pm 0.06$ & $12.21 \pm 0.06$ & $12.08 \pm 0.06$ & $10.66 \pm 0.28$ & $>1.05$ & $\ldots$ & & Yes & No & No & No & $\ldots$ \\
\hline $042745.3+235724$ & CFHT-15 & $13.24 \pm 0.06$ & $13.15 \pm 0.06$ & $13.25 \pm 0.07$ & $13.05 \pm 0.10$ & $>10.55$ & $>1.06$ & $\ldots$ & $\ldots$ & & & No & No & $\ldots$ \\
\hline $042757.3+261918$ & IRAS04248+2612 AB & $9.83 \pm 0.06$ & $9.10 \pm 0.05$ & $8.28 \pm 0.05$ & $7.10 \pm 0.05$ & $2.27 \pm 0.04$ & $-1.52 \pm 0.22$ & $-4.39 \pm 0.34$ & Yes & Yes & Yes & Yes & Yes & $\ldots$ \\
\hline $042838.9+265135$ & LDN 1521F-IRS & $15.33 \pm 0.08$ & $14.25 \pm 0.07$ & $13.45 \pm 0.10$ & $12.04 \pm 0.07$ & $6.16 \pm 0.04$ & $0.57 \pm 0.22$ & $-4.28 \pm 0.34$ & Yes & $\ldots$ & Yes-faint & No & No & $\ldots$ \\
\hline $042842.6+271403$ & $\mathrm{~J} 04284263+2714039 \mathrm{AB}$ & $9.76 \pm 0.05$ & $9.53 \pm 0.05$ & $9.21 \pm 0.05$ & $8.83 \pm 0.05$ & $6.21 \pm 0.05$ & $>0.88$ & $\ldots$ & $\ldots$ & Yes & Yes & Yes & Yes & . \\
\hline $042900.6+275503$ & J04290068+2755033 & $12.30 \pm 0.05$ & $11.99 \pm 0.05$ & $11.60 \pm 0.06$ & $10.92 \pm 0.06$ & $8.06 \pm 0.07$ & $>0.88$ & $\ldots$ & & Yes & No & No & Yes & $\ldots$ \\
\hline $042904.9+264907$ & IRAS04260+2642 & $10.08 \pm 0.05$ & $9.40 \pm 0.05$ & $8.83 \pm 0.05$ & $8.07 \pm 0.05$ & $3.60 \pm 0.04$ & $0.06 \pm 0.22$ & $\ldots$ & Yes & Yes & Yes & Yes & Yes & $\ldots$ \\
\hline $042920.7+263340$ & $\mathrm{~J} 1-507$ & $8.56 \pm 0.05$ & $8.55 \pm 0.05$ & $8.47 \pm 0.05$ & $8.46 \pm 0.05$ & $8.29 \pm 0.10$ & $>1.04$ & $\ldots$ & $\ldots$ & No & No & No & No & $\ldots$ \\
\hline $042921.6+270125$ & IRAS04263+2654 & $8.06 \pm 0.05$ & $7.67 \pm 0.05$ & $7.31 \pm 0.05$ & $6.69 \pm 0.05$ & $3.41 \pm 0.04$ & $1.11 \pm 0.22$ & $\ldots$ & Yes & Yes & Yes & Yes & Yes & $\ldots$ \\
\hline $042923.7+243300$ & GV Tau AB & $<6.62$ & $<6.10$ & $<3.49$ & $<3.52$ & $<0.45$ & $<-2.30$ & $<-1.49$ & $\ldots$ & $\ldots$ & $\ldots$ & $\ldots$ & $\ldots$ & $\mathrm{cd}$ \\
\hline $042929.7+261653$ & FW Tau ABC & $9.09 \pm 0.05$ & $9.01 \pm 0.05$ & $8.88 \pm 0.05$ & $8.88 \pm 0.05$ & $7.52 \pm 0.06$ & $>1.07$ & $\ldots$ & $\ldots$ & Yes & Yes & No & No & $\ldots$ \\
\hline $042930.0+243955$ & IRAS04264+2433 & $10.21 \pm 0.05$ & $9.43 \pm 0.05$ & $8.60 \pm 0.05$ & $6.72 \pm 0.05$ & $1.12 \pm 0.04$ & $-1.37 \pm 0.22$ & $>-1.94$ & Yes & Yes & Yes & Yes & Yes & $\ldots$ \\
\hline $042941.5+263258$ & DH Tau AB & $7.63 \pm 0.05$ & $7.33 \pm 0.05$ & $7.20 \pm 0.05$ & $6.86 \pm 0.05$ & $3.37 \pm 0.04$ & $0.82 \pm 0.22$ & $\ldots$ & Yes & Yes & Yes & Yes & Yes & $\ldots$ \\
\hline $042942.4+263249$ & DI Tau AB & $8.21 \pm 0.05$ & $8.22 \pm 0.05$ & $8.14 \pm 0.05$ & $8.11 \pm 0.05$ & $\ldots$ & $>0.72$ & $\ldots$ & $\ldots$ & $\ldots$ & $\ldots$ & No & No & $\ldots$ \\
\hline $042945.6+263046$ & KPNO-5 & $11.05 \pm 0.05$ & $11.02 \pm 0.05$ & $10.94 \pm 0.06$ & $10.83 \pm 0.06$ & $>9.71$ & $>0.90$ & $\ldots$ & $\ldots$ & $\ldots$ & $\ldots$ & No & No & $\ldots$ \\
\hline $042951.5+260644$ & IQ Tau & $6.81 \pm 0.05$ & $6.37 \pm 0.05$ & $6.07 \pm 0.05$ & $5.53 \pm 0.05$ & $2.82 \pm 0.04$ & $0.32 \pm 0.22$ & $\ldots$ & Yes & Yes & Yes & Yes & Yes & $\ldots$ \\
\hline $042959.5+243307$ & CFHT-20 & $9.02 \pm 0.05$ & $8.55 \pm 0.05$ & $8.32 \pm 0.05$ & $7.84 \pm 0.05$ & $4.91 \pm 0.04$ & $2.06 \pm 0.22$ & $\ldots$ & Yes & Yes & Yes & Yes & Yes & $\ldots$ \\
\hline $043007.2+260820$ & KPNO-6 & $13.12 \pm 0.06$ & $12.77 \pm 0.06$ & $12.42 \pm 0.06$ & $11.58 \pm 0.06$ & $9.20 \pm 0.19$ & $>1.01$ & $\ldots$ & $\ldots$ & Yes-faint & No & No & Yes & $\ldots$ \\
\hline $043023.6+235912$ & CFHT-16 & $13.23 \pm 0.06$ & $13.15 \pm 0.06$ & $13.04 \pm 0.08$ & $12.99 \pm 0.09$ & $>10.54$ & $>1.00$ & $\ldots$ & $\ldots$ & & & No & No & $\ldots$ \\
\hline $043029.6+242645$ & FX Tau AB & $7.22 \pm 0.05$ & $6.96 \pm 0.05$ & $6.69 \pm 0.05$ & $5.97 \pm 0.05$ & $3.03 \pm 0.04$ & $1.09 \pm 0.22$ & & Yes & Yes & Yes & Yes & Yes & $\ldots$ \\
\hline $043044.2+260124$ & DK Tau AB & $<6.62$ & $<6.10$ & $5.52 \pm 0.05$ & $4.78 \pm 0.05$ & $1.85 \pm 0.04$ & $0.08 \pm 0.22$ & $0.57 \pm 0.22$ & Yes & Yes & Yes & $\ldots$ & $\ldots$ & $\ldots$ \\
\hline $043050.2+230008$ & IRAS04278+2253 & $<6.62$ & $<6.10$ & $<3.49$ & $<3.52$ & $<0.45$ & $-1.87 \pm 0.22$ & $-3.88 \pm 0.34$ & $\ldots$ & $\ldots$ & $\ldots$ & $\ldots$ & $\ldots$ & $\ldots$ \\
\hline $043051.3+244222$ & ZZ Tau AB & $8.08 \pm 0.05$ & $7.90 \pm 0.05$ & $7.61 \pm 0.05$ & $6.94 \pm 0.05$ & $4.53 \pm 0.04$ & $>0.72$ & $>-4.46$ & $\ldots$ & Yes & Yes & Yes & Yes & $\mathrm{b}$ \\
\hline $043051.7+244147$ & ZZ Tau IRS & $8.11 \pm 0.05$ & $7.38 \pm 0.05$ & $6.73 \pm 0.05$ & $5.78 \pm 0.05$ & $2.01 \pm 0.04$ & $-0.99 \pm 0.22$ & $-3.61 \pm 0.22$ & Yes & Yes & Yes & Yes & Yes & $\mathrm{b}$ \\
\hline $043057.1+255639$ & KPNO-7 & $12.62 \pm 0.05$ & $12.28 \pm 0.05$ & $11.99 \pm 0.06$ & $11.25 \pm 0.06$ & $8.62 \pm 0.12$ & $>1.23$ & $\ldots$ & & Yes & No & No & Yes & $\ldots$ \\
\hline $043114.4+271017$ & JH56 & $8.72 \pm 0.05$ & $8.75 \pm 0.05$ & $8.66 \pm 0.05$ & $8.60 \pm 0.05$ & $6.76 \pm 0.02$ & $>0.75$ & $\ldots$ & & Yes & Yes & No & No & \\
\hline $043119.0+233504$ & $\mathrm{~J} 04311907+2335047$ & $11.66 \pm 0.05$ & $11.53 \pm 0.05$ & $11.56 \pm 0.06$ & $11.46 \pm 0.06$ & $>10.59$ & $>0.86$ & $\ldots$ & & & & No & No & $\ldots$ \\
\hline $043123.8+241052$ & V927 Tau AB & $8.52 \pm 0.05$ & $8.47 \pm 0.05$ & $8.38 \pm 0.05$ & $8.38 \pm 0.05$ & $8.19 \pm 0.09$ & $>0.91$ & $\ldots$ & $\ldots$ & No & No & No & No & \\
\hline $043126.6+270318$ & CFHT-13 & $12.90 \pm 0.06$ & $12.75 \pm 0.06$ & $12.72 \pm 0.07$ & $12.70 \pm 0.07$ & $10.72 \pm 0.29$ & $>0.68$ & $\ldots$ & $\ldots$ & Yes & No & No & No & $\ldots$ \\
\hline $043150.5+242418$ & HK Tau AB & $7.71 \pm 0.05$ & $7.35 \pm 0.05$ & $7.10 \pm 0.05$ & $6.58 \pm 0.05$ & $2.31 \pm 0.04$ & $-0.81 \pm 0.22$ & $-3.02 \pm 0.22$ & Yes & Yes & Yes & Yes & Yes & $\ldots$ \\
\hline $043158.4+254329$ & $\mathrm{~J} 1-665$ & $9.35 \pm 0.05$ & $9.29 \pm 0.05$ & $9.24 \pm 0.05$ & $9.22 \pm 0.05$ & $9.04 \pm 0.17$ & $>1.08$ & $\ldots$ & $\ldots$ & No & No & No & No & $\ldots$ \\
\hline $043203.2+252807$ & J04320329+2528078 & $10.30 \pm 0.05$ & $10.20 \pm 0.05$ & $10.13 \pm 0.06$ & $10.09 \pm 0.06$ & $>9.67$ & $>1.03$ & $\ldots$ & $\ldots$ & & & No & No & $\ldots$ \\
\hline $043215.4+242859$ & Haro6-13 & $<6.62$ & $<6.10$ & $5.49 \pm 0.05$ & $4.85 \pm 0.05$ & $0.88 \pm 0.04$ & $-1.43 \pm 0.22$ & $-4.02 \pm 0.34$ & Yes & Yes & Yes & $\ldots$ & $\ldots$ & $\ldots$ \\
\hline $043217.8+242214$ & CFHT-7 AB & $9.98 \pm 0.05$ & $9.87 \pm 0.05$ & $9.76 \pm 0.05$ & $9.72 \pm 0.05$ & $9.30 \pm 0.28$ & $>0.86$ & $\ldots$ & $\ldots$ & Yes & No & No & No & $\ldots$ \\
\hline $043218.8+242227$ & V928 Tau AB & $7.86 \pm 0.05$ & $7.82 \pm 0.05$ & $7.72 \pm 0.05$ & $7.64 \pm 0.05$ & $7.54 \pm 0.06$ & $>0.84$ & $\ldots$ & $\ldots$ & No & No & No & No & $\ldots$ \\
\hline $043223.2+240301$ & $\mathrm{~J} 04322329+2403013$ & $10.89 \pm 0.05$ & $10.83 \pm 0.05$ & $10.79 \pm 0.06$ & $10.67 \pm 0.06$ & $>9.82$ & $>0.91$ & $\ldots$ & $\ldots$ & $\ldots$ & $\ldots$ & No & No & $\ldots$ \\
\hline $043230.5+241957$ & FY Tau & $7.18 \pm 0.05$ & $6.76 \pm 0.05$ & $6.50 \pm 0.05$ & $5.99 \pm 0.05$ & $3.67 \pm 0.04$ & $\ldots$ & $\ldots$ & $\ldots$ & Yes & Yes & Yes & Yes & $\ldots$ \\
\hline $043231.7+242002$ & FZ Tau & $<6.62$ & $<6.10$ & $5.27 \pm 0.05$ & $4.58 \pm 0.05$ & $2.06 \pm 0.04$ & $0.31 \pm 0.22$ & $\ldots$ & Yes & Yes & Yes & .. & & $\ldots$ \\
\hline $043232.0+225726$ & IRAS04295+2251 & $8.63 \pm 0.05$ & $7.72 \pm 0.05$ & $6.83 \pm 0.05$ & $5.32 \pm 0.05$ & $1.40 \pm 0.04$ & $-1.32 \pm 0.22$ & $-3.93 \pm 0.34$ & Yes & Yes & Yes & Yes & Yes & $\ldots$ \\
\hline $043243.0+255231$ & UZ Tau Aab & $<6.62$ & $<6.10$ & $5.63 \pm 0.05$ & $4.79 \pm 0.05$ & $1.54 \pm 0.04$ & $-0.69 \pm 0.22$ & $-2.15 \pm 0.22$ & Yes & Yes & Yes & $\ldots$ & $\ldots$ & $\mathrm{b}$ \\
\hline $043249.1+225302$ & JH112 & $7.41 \pm 0.05$ & $7.12 \pm 0.05$ & $6.83 \pm 0.05$ & $5.89 \pm 0.05$ & $2.53 \pm 0.04$ & $0.72 \pm 0.22$ & $\ldots$ & Yes & Yes & Yes & Yes & Yes & $\ldots$ \\
\hline $043250.2+242211$ & CFHT-5 & $10.46 \pm 0.05$ & $10.27 \pm 0.05$ & $10.09 \pm 0.06$ & $10.07 \pm 0.06$ & $9.56 \pm 0.29$ & $>1.16$ & $>-1.44$ & $\ldots$ & Yes & No & No & No & $\ldots$ \\
\hline $043301.9+242100$ & MHO-8 & $9.32 \pm 0.05$ & $9.21 \pm 0.05$ & $9.14 \pm 0.05$ & $9.09 \pm 0.05$ & $8.92 \pm 0.15$ & $>0.88$ & $\ldots$ & $\ldots$ & No & No & No & No & 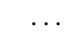 \\
\hline
\end{tabular}




\begin{tabular}{|c|c|c|c|c|c|c|c|c|c|c|c|c|c|c|}
\hline SST Tau Name & Common Name & $\begin{array}{l}{[3.6]} \\
\text { (mag) }\end{array}$ & $\begin{array}{l}{[4.5]} \\
\text { (mag) }\end{array}$ & $\begin{array}{c}{[5.8]} \\
(\mathrm{mag})\end{array}$ & $\begin{array}{c}{[8]} \\
(\mathrm{mag})\end{array}$ & $\begin{array}{c}{[24]} \\
\text { (mag) }\end{array}$ & $\begin{array}{c}{[70]} \\
(\mathrm{mag})\end{array}$ & $\begin{array}{l}{[160]} \\
\text { (mag) }\end{array}$ & $\begin{array}{l}\text { Ident. in } \\
24 / 70 \\
\text { CMD }\end{array}$ & $\begin{array}{c}\text { Ident. in } \\
K_{\mathrm{S}} / 24 \\
\mathrm{CMD}\end{array}$ & $\begin{array}{c}\text { Ident. in } \\
8 / 24 \\
\text { CMD }\end{array}$ & $\begin{array}{c}\text { Ident. in } \\
4.5 / 8 \\
\text { CMD }\end{array}$ & $\begin{array}{l}\text { Ident. in } \\
\text { IRAC } \\
\text { CCD }\end{array}$ & Notes \\
\hline $043306.2+240933$ & GH Tau AB & $7.08 \pm 0.05$ & $6.77 \pm 0.05$ & $6.50 \pm 0.05$ & $6.03 \pm 0.05$ & $3.17 \pm 0.04$ & $0.43 \pm 0.22$ & & Yes & Yes & Yes & Yes & Yes & $\cdots$ \\
\hline $043306.6+240954$ & V807 Tau AB & $<6.62$ & $6.21 \pm 0.05$ & $5.96 \pm 0.05$ & $5.57 \pm 0.05$ & $2.96 \pm 0.04$ & $0.36 \pm 0.22$ & $\ldots$ & Yes & Yes & Yes & Yes & $\ldots$ & $\ldots$ \\
\hline $043307.8+261606$ & KPNO-14 & $9.78 \pm 0.05$ & $9.67 \pm 0.06$ & $9.60 \pm 0.05$ & $9.58 \pm 0.05$ & $9.04 \pm 0.12$ & $>1.44$ & $>-1.91$ & & Yes & Yes-faint & No & No & $\ldots$ \\
\hline $043309.4+224648$ & CFHT-12 & $10.86 \pm 0.05$ & $10.63 \pm 0.05$ & $10.34 \pm 0.06$ & $9.95 \pm 0.06$ & $8.25 \pm 0.07$ & $>1.16$ & $\ldots$ & & Yes & Yes-faint & Yes & Yes & $\ldots$ \\
\hline $043310.0+243343$ & V830 Tau & $8.41 \pm 0.05$ & $8.41 \pm 0.05$ & $8.37 \pm 0.05$ & $8.32 \pm 0.05$ & $8.14 \pm 0.08$ & $>1.03$ & $\ldots$ & $\ldots$ & No & No & No & No & $\ldots$ \\
\hline $043314.3+261423$ & IRAS04301+2608 & $12.05 \pm 0.05$ & $11.72 \pm 0.05$ & $11.29 \pm 0.06$ & $9.54 \pm 0.05$ & $3.28 \pm 0.04$ & $1.12 \pm 0.22$ & $>-0.95$ & Yes & Yes & Yes-faint & Yes-faint & Yes & $\ldots$ \\
\hline $043316.5+225320$ & IRAS04302+2247 & $10.29 \pm 0.05$ & $9.88 \pm 0.05$ & $9.72 \pm 0.05$ & $9.71 \pm 0.06$ & $3.57 \pm 0.04$ & $-1.88 \pm 0.22$ & $-4.51 \pm 0.34$ & Yes & Yes & Yes-faint & No & No & $\ldots$ \\
\hline $043319.0+224634$ & IRAS04303+2240 & $<6.62$ & $<6.10$ & $4.77 \pm 0.05$ & $3.73 \pm 0.05$ & $1.43 \pm 0.04$ & $-0.11 \pm 0.22$ & $\ldots$ & Yes & Yes & Yes & $\ldots$ & $\ldots$ & $\ldots$ \\
\hline $043334.0+242117$ & GI Tau & $6.87 \pm 0.05$ & $6.31 \pm 0.05$ & $5.79 \pm 0.05$ & $5.12 \pm 0.05$ & $2.15 \pm 0.04$ & & $\ldots$ & $\ldots$ & Yes & Yes & Yes & Yes & $\ldots$ \\
\hline $043334.5+242105$ & GK Tau & $<6.62$ & $<6.10$ & $5.79 \pm 0.05$ & $5.14 \pm 0.05$ & $1.70 \pm 0.04$ & $-0.23 \pm 0.22$ & $\ldots$ & Yes & Yes & Yes & $\ldots$ & $\ldots$ & $\ldots$ \\
\hline $043336.7+260949$ & IS Tau AB & $7.85 \pm 0.05$ & $7.46 \pm 0.05$ & $6.94 \pm 0.05$ & $6.03 \pm 0.05$ & $3.65 \pm 0.04$ & $2.08 \pm 0.22$ & $>-0.83$ & Yes & Yes & Yes & Yes & Yes & $\ldots$ \\
\hline $043339.0+252038$ & DL Tau & $6.95 \pm 0.05$ & $6.37 \pm 0.05$ & $5.92 \pm 0.05$ & $5.13 \pm 0.05$ & $2.19 \pm 0.04$ & $-0.25 \pm 0.22$ & $-2.44 \pm 0.22$ & Yes & Yes & Yes & Yes & Yes & $\ldots$ \\
\hline $043342.9+252647$ & J04334291+2526470 & $12.76 \pm 0.06$ & $12.63 \pm 0.06$ & $12.52 \pm 0.07$ & $12.47 \pm 0.07$ & $>11.05$ & $>1.43$ & $\cdots$ & $\cdots$ & $\cdots$ & $\cdots$ & No & No & $\cdots$ \\
\hline $043352.0+225030$ & CI Tau & $6.99 \pm 0.05$ & $6.53 \pm 0.05$ & $6.17 \pm 0.05$ & $5.33 \pm 0.05$ & $2.37 \pm 0.04$ & $-0.80 \pm 0.22$ & $\ldots$ & Yes & Yes & Yes & Yes & Yes & $\ldots$ \\
\hline $043352.5+225626$ & 2MASS J04335252+2256269 & $8.79 \pm 0.05$ & $8.71 \pm 0.05$ & $8.63 \pm 0.05$ & $8.60 \pm 0.05$ & $8.32 \pm 0.09$ & $>1.41$ & $\ldots$ & & No & No & No & No & $\ldots$ \\
\hline $043354.7+261327$ & IT Tau AB & $7.35 \pm 0.05$ & $6.98 \pm 0.05$ & $6.63 \pm 0.05$ & $6.05 \pm 0.05$ & $3.53 \pm 0.04$ & $0.83 \pm 0.22$ & $>-1.28$ & Yes & Yes & Yes & Yes & Yes & $\ldots$ \\
\hline $043410.9+225144$ & JH108 & $9.30 \pm 0.05$ & $9.27 \pm 0.05$ & $9.19 \pm 0.05$ & $9.17 \pm 0.05$ & $8.88 \pm 0.12$ & $>1.15$ & $\ldots$ & $\ldots$ & No & No & No & No & $\ldots$ \\
\hline $043415.2+225030$ & CFHT-1 & $11.23 \pm 0.05$ & $11.10 \pm 0.05$ & $10.98 \pm 0.06$ & $11.02 \pm 0.06$ & $>9.94$ & $>1.00$ & $\ldots$ & $\ldots$ & $\ldots$ & $\ldots$ & No & No & $\ldots$ \\
\hline $043439.2+250101$ & Wa Tau 1 & $7.83 \pm 0.05$ & $7.79 \pm 0.05$ & $7.75 \pm 0.05$ & $7.73 \pm 0.05$ & $7.67 \pm 0.07$ & $>0.85$ & $\ldots$ & $\ldots$ & No & No & No & No & $\ldots$ \\
\hline $043455.4+242853$ & AA Tau & $7.29 \pm 0.05$ & $6.84 \pm 0.05$ & $6.44 \pm 0.05$ & $5.65 \pm 0.05$ & $2.81 \pm 0.04$ & $-0.14 \pm 0.22$ & $-2.47 \pm 0.22$ & Yes & Yes & Yes & Yes & Yes & $\ldots$ \\
\hline $043508.5+231139$ & CFHT-11 & $11.19 \pm 0.05$ & $11.12 \pm 0.05$ & $11.04 \pm 0.06$ & $10.99 \pm 0.06$ & $10.23 \pm 0.20$ & $>0.37$ & $\ldots$ & $\ldots$ & Yes & No & No & No & $\ldots$ \\
\hline $043520.2+223214$ & HO Tau & $8.90 \pm 0.05$ & $8.52 \pm 0.05$ & $8.38 \pm 0.05$ & $7.73 \pm 0.05$ & $4.85 \pm 0.04$ & $2.43 \pm 0.22$ & $\ldots$ & Yes & Yes & Yes & Yes & Yes & $\ldots$ \\
\hline $043520.8+225424$ & FF Tau AB & $8.45 \pm 0.05$ & $8.44 \pm 0.05$ & $8.42 \pm 0.05$ & $8.36 \pm 0.05$ & $8.15 \pm 0.09$ & $>1.26$ & $\ldots$ & $\ldots$ & No & No & No & No & $\ldots$ \\
\hline $043527.3+241458$ & DN Tau & $7.47 \pm 0.05$ & $7.16 \pm 0.05$ & $6.78 \pm 0.05$ & $6.03 \pm 0.05$ & $3.04 \pm 0.04$ & $0.44 \pm 0.22$ & $>0.04$ & Yes & Yes & Yes & Yes & Yes & $\ldots$ \\
\hline $043535.3+240819$ & IRAS04325+2402 A & $9.93 \pm 0.05$ & $9.28 \pm 0.05$ & $9.06 \pm 0.05$ & $8.54 \pm 0.05$ & $1.43 \pm 0.04$ & $-2.26 \pm 0.22$ & $-5.07 \pm 0.34$ & Yes & Yes & Yes & Yes & Yes & $\ldots$ \\
\hline $043540.9+241108$ & CoKu Tau/3 AB & $7.43 \pm 0.05$ & $6.95 \pm 0.05$ & $6.48 \pm 0.05$ & $5.64 \pm 0.05$ & $3.31 \pm 0.04$ & $1.33 \pm 0.22$ & $>-2.14$ & Yes & Yes & Yes & Yes & Yes & $\ldots$ \\
\hline $043541.8+223411$ & KPNO-8 & $11.64 \pm 0.05$ & $11.54 \pm 0.05$ & $11.43 \pm 0.06$ & $11.46 \pm 0.06$ & $>10.71$ & $>1.12$ & $\ldots$ & $\ldots$ & $\ldots$ & $\ldots$ & No & No & $\ldots$ \\
\hline $043545.2+273713$ & J04354526+2737130 & $13.18 \pm 0.06$ & $13.11 \pm 0.06$ & $12.93 \pm 0.07$ & $13.07 \pm 0.11$ & $>10.62$ & $>1.47$ & $\ldots$ & $\ldots$ & $\ldots$ & $\ldots$ & No & No & $\ldots$ \\
\hline $043547.3+225021$ & HQ Tau & $<6.62$ & $<6.10$ & $5.61 \pm 0.05$ & $4.47 \pm 0.05$ & $1.65 \pm 0.04$ & $-0.18 \pm 0.22$ & $\ldots$ & Yes & Yes & Yes & $\ldots$ & $\ldots$ & $\ldots$ \\
\hline $043551.0+225240$ & KPNO-15 & $9.79 \pm 0.05$ & $9.72 \pm 0.05$ & $9.66 \pm 0.05$ & $9.65 \pm 0.05$ & $9.71 \pm 0.11$ & $>0.72$ & $\ldots$ & $\ldots$ & No & No & No & No & $\ldots$ \\
\hline $043551.4+224911$ & KPNO-9 & $13.63 \pm 0.06$ & $13.52 \pm 0.06$ & $13.70 \pm 0.12$ & $13.41 \pm 0.17$ & $>10.93$ & $>0.86$ & $\ldots$ & $\ldots$ & $\ldots$ & $\ldots$ & No & No & $\ldots$ \\
\hline $043552.0+225503$ & 2MASS J04355209+2255039 & $9.56 \pm 0.05$ & $9.52 \pm 0.05$ & $9.39 \pm 0.05$ & $9.35 \pm 0.06$ & $\ldots$ & $>0.50$ & $\cdots$ & $\ldots$ & $\ldots$ & .. & No & No & $\ldots$ \\
\hline $043552.7+225423$ & HP Tau AB & $<6.62$ & $6.20 \pm 0.05$ & $5.65 \pm 0.05$ & $4.88 \pm 0.05$ & $1.49 \pm 0.04$ & $-1.93 \pm 0.22$ & $-4.48 \pm 0.34$ & Yes & Yes & Yes & Yes & $\cdots$ & $\ldots$ \\
\hline $043552.8+225058$ & 2MASS J04355286+2250585 & $9.46 \pm 0.05$ & $9.36 \pm 0.05$ & $9.28 \pm 0.05$ & $9.29 \pm 0.05$ & $9.10 \pm 0.13$ & $>0.93$ & .. & $\ldots$ & No & No & No & No & $\ldots$ \\
\hline $043553.4+225408$ & HP Tau/G3 AB & $8.62 \pm 0.05$ & $8.60 \pm 0.05$ & $8.51 \pm 0.05$ & $8.47 \pm 0.06$ & $\ldots$ & $>-0.03$ & $>-2.19$ & $\ldots$ & $\ldots$ & $\ldots$ & No & No & $\ldots$ \\
\hline $043554.1+225413$ & HP Tau/G2 & $7.19 \pm 0.05$ & $7.17 \pm 0.05$ & $7.11 \pm 0.05$ & $7.02 \pm 0.05$ & $\ldots$ & $>-0.03$ & $>-2.35$ & $\ldots$ & $\ldots$ & $\ldots$ & No & No & $\ldots$ \\
\hline $043556.8+225436$ & Haro 6-28 AB & $8.61 \pm 0.05$ & $8.18 \pm 0.05$ & $7.85 \pm 0.05$ & $7.14 \pm 0.05$ & $4.39 \pm 0.04$ & $>0.70$ & $>-2.24$ & $\ldots$ & Yes & Yes & Yes & Yes & $\cdots$ \\
\hline $043558.9+223835$ & 2MASS J04355892+2238353 & $8.15 \pm 0.05$ & $8.19 \pm 0.05$ & $8.10 \pm 0.05$ & $8.06 \pm 0.05$ & $\ldots$ & $>1.12$ & $\ldots$ & $\ldots$ & $\ldots$ & $\ldots$ & No & No & $\ldots$ \\
\hline $043610.3+215936$ & J04361030+2159364 & $13.02 \pm 0.06$ & $12.74 \pm 0.06$ & $12.41 \pm 0.06$ & $11.74 \pm 0.06$ & $9.01 \pm 0.18$ & $>1.07$ & $\ldots$ & $\ldots$ & Yes-faint & No & No & Yes & $\cdots$ \\
\hline $043610.3+225956$ & CFHT-2 & $11.63 \pm 0.05$ & $11.43 \pm 0.05$ & $11.34 \pm 0.06$ & $11.32 \pm 0.06$ & $>10.61$ & $>1.48$ & $>-3.51$ & $\ldots$ & $\ldots$ & $\ldots$ & No & No & $\ldots$ \\
\hline $043619.0+254258$ & LkCa 14 & $8.52 \pm 0.05$ & $8.54 \pm 0.05$ & $8.51 \pm 0.05$ & $8.45 \pm 0.05$ & $8.24 \pm 0.10$ & $>0.99$ & $>-0.98$ & $\ldots$ & No & No & No & No & $\ldots$ \\
\hline $043638.9+225811$ & CFHT-3 & $11.79 \pm 0.05$ & $11.69 \pm 0.05$ & $11.59 \pm 0.06$ & $11.57 \pm 0.06$ & $>8.55$ & $>1.12$ & $\ldots$ & $\ldots$ & $\ldots$ & $\ldots$ & No & No & $\cdots$ \\
\hline $043649.1+241258$ & HD 283759 & $8.32 \pm 0.05$ & $8.25 \pm 0.05$ & $8.30 \pm 0.05$ & $8.20 \pm 0.05$ & $6.64 \pm 0.05$ & $1.10 \pm 0.22$ & $>0.51$ & Yes & Yes & Yes & No & No & . \\
\hline $043800.8+255857$ & ITG 2 & $9.60 \pm 0.05$ & $9.47 \pm 0.05$ & $9.37 \pm 0.05$ & $9.31 \pm 0.05$ & $9.17 \pm 0.19$ & $>0.96$ & $>-2.05$ & $\ldots$ & No & No & No & No & \\
\hline $043814.8+261139$ & J04381486+2611399 & $10.80 \pm 0.05$ & $10.21 \pm 0.05$ & $9.64 \pm 0.05$ & $8.92 \pm 0.05$ & $4.98 \pm 0.04$ & $>0.80$ & $>-1.11$ & $\cdots$ & Yes & Yes & Yes & Yes & \\
\hline
\end{tabular}


Table 4

\begin{tabular}{|c|c|c|c|c|c|c|c|c|c|c|c|c|c|c|}
\hline SST Tau Name & Common Name & $\begin{array}{l}{[3.6]} \\
\text { (mag) }\end{array}$ & $\begin{array}{l}{[4.5]} \\
\text { (mag) }\end{array}$ & $\begin{array}{l}{[5.8]} \\
\text { (mag) }\end{array}$ & $\begin{array}{c}{[8]} \\
(\mathrm{mag})\end{array}$ & $\begin{array}{c}{[24]} \\
(\mathrm{mag})\end{array}$ & $\begin{array}{c}{[70]} \\
(\mathrm{mag})\end{array}$ & $\begin{array}{l}{[160]} \\
\text { (mag) }\end{array}$ & $\begin{array}{l}\text { Ident. in } \\
24 / 70 \\
\text { CMD }\end{array}$ & $\begin{array}{c}\text { Ident. in } \\
K_{\mathrm{S}} / 24 \\
\mathrm{CMD}\end{array}$ & $\begin{array}{c}\text { Ident. in } \\
8 / 24 \\
\text { CMD }\end{array}$ & $\begin{array}{c}\text { Ident. in } \\
4.5 / 8 \\
\text { CMD }\end{array}$ & $\begin{array}{l}\text { Ident. in } \\
\text { IRAC } \\
\text { CCD }\end{array}$ & Notes \\
\hline $043815.6+230227$ & RXJ0438.2+2302 & $9.69 \pm 0.05$ & $9.69 \pm 0.05$ & $9.64 \pm 0.05$ & $9.60 \pm 0.05$ & $>9.35$ & $>1.08$ & & & & & No & No & \\
\hline $043821.3+260913$ & GM Tau & $9.27 \pm 0.05$ & $8.77 \pm 0.05$ & $8.43 \pm 0.05$ & $7.81 \pm 0.05$ & $5.33 \pm 0.04$ & $>0.97$ & $>-1.31$ & & Yes & Yes & Yes & Yes & \\
\hline $043828.5+261049$ & DO Tau & $<6.62$ & $<6.10$ & $5.26 \pm 0.05$ & $4.77 \pm 0.05$ & $1.09 \pm 0.04$ & $-1.37 \pm 0.22$ & $-3.92 \pm 0.34$ & Yes & Yes & Yes & $\ldots$ & $\ldots$ & \\
\hline $043835.2+261038$ & HV Tau AB & $7.65 \pm 0.05$ & $7.59 \pm 0.05$ & $7.49 \pm 0.05$ & $7.46 \pm 0.05$ & $\ldots$ & $>0.72$ & $>-3.65$ & $\ldots$ & $\ldots$ & $\ldots$ & No & No & \\
\hline $043835.4+261041$ & HV Tau C & $11.33 \pm 0.14$ & $10.74 \pm 0.05$ & $10.22 \pm 0.05$ & $9.38 \pm 0.04$ & $3.52 \pm 0.04$ & $-0.09 \pm 0.22$ & $\ldots$ & Yes & No & Yes & Yes & Yes & $\mathrm{e}$ \\
\hline $043858.5+233635$ & J0438586+2336352 & $10.51 \pm 0.05$ & $\ldots$ & $9.84 \pm 0.05$ & $\cdots$ & $6.39 \pm 0.05$ & $>0.85$ & $\cdots$ & $\ldots$ & Yes & $\ldots$ & $\ldots$ & $\ldots$ & $\ldots$ \\
\hline $043901.6+233602$ & $\mathrm{~J} 0439016+2336030$ & $9.76 \pm 0.05$ & $\ldots$ & $9.18 \pm 0.05$ & $\ldots$ & $6.28 \pm 0.05$ & $>2.30$ & $\ldots$ & $\ldots$ & Yes & $\ldots$ & $\ldots$ & $\ldots$ & $\ldots$ \\
\hline $043903.9+254426$ & CFHT-6 & $10.75 \pm 0.05$ & $10.45 \pm 0.05$ & $10.02 \pm 0.06$ & $9.14 \pm 0.05$ & $6.51 \pm 0.05$ & $>0.47$ & $>-0.54$ & $\ldots$ & Yes & Yes & Yes & Yes & $\mathrm{c}$ \\
\hline $043906.3+233417$ & J0439064+2334179 & $10.73 \pm 0.05$ & $\ldots$ & $10.62 \pm 0.06$ & $\ldots$ & $>9.32$ & $\ldots$ & $\ldots$ & $\ldots$ & $\ldots$ & $\ldots$ & $\ldots$ & $\ldots$ & $\ldots$ \\
\hline $043913.8+255320$ & IRAS04361+2547 AB & $8.00 \pm 0.05$ & $7.08 \pm 0.05$ & $6.46 \pm 0.05$ & $4.82 \pm 0.05$ & $<0.45$ & $<-2.30$ & $<-4.73$ & $\ldots$ & $\ldots$ & $\ldots$ & Yes & Yes & $\ldots$ \\
\hline $043917.7+222103$ & LkCa 15 & $7.61 \pm 0.05$ & $7.41 \pm 0.05$ & $7.23 \pm 0.05$ & $6.64 \pm 0.05$ & $3.11 \pm 0.04$ & $-0.40 \pm 0.22$ & $-2.47 \pm 0.22$ & Yes & Yes & Yes & Yes & Yes & $\ldots$ \\
\hline $043920.9+254502$ & GN Tau B & $6.99 \pm 0.05$ & $6.58 \pm 0.05$ & $6.21 \pm 0.05$ & $5.42 \pm 0.05$ & $2.82 \pm 0.04$ & $1.59 \pm 0.22$ & $>-2.43$ & Yes & Yes & Yes & Yes & Yes & $\ldots$ \\
\hline $043935.1+254144$ & IRAS04365+2535 & $7.22 \pm 0.05$ & $<6.10$ & $4.87 \pm 0.05$ & $4.16 \pm 0.05$ & $<0.45$ & $-2.17 \pm 0.22$ & $<-3.82$ & $\ldots$ & & $\ldots$ & & .. & $\mathrm{c}$ \\
\hline $043947.4+260140$ & CFHT-4 & $9.54 \pm 0.05$ & $9.07 \pm 0.05$ & $8.60 \pm 0.05$ & $7.78 \pm 0.05$ & $4.95 \pm 0.04$ & $>0.91$ & $>-4.76$ & $\ldots$ & Yes & Yes & Yes & Yes & \\
\hline $043953.9+260309$ & IRAS $04368+2557$ & $13.39 \pm 0.11$ & $11.15 \pm 0.08$ & $10.09 \pm 0.07$ & $9.73 \pm 0.08$ & $2.69 \pm 0.04$ & $<-2.30$ & $<-4.40$ & $\ldots$ & $\ldots$ & Yes-faint & Yes-faint & Yes & $\mathrm{cd}$ \\
\hline $043955.7+254502$ & IC2087 IRS & $<6.62$ & $<6.10$ & $<3.49$ & $<3.52$ & $<0.45$ & $-2.17 \pm 0.22$ & $>-5.41$ & $\ldots$ & $\ldots$ & $\cdots$ & $\ldots$ & $\ldots$ & $\mathrm{c}$ \\
\hline $044001.7+255629$ & CFHT-17 AB & $10.15 \pm 0.05$ & $9.96 \pm 0.05$ & $9.87 \pm 0.05$ & $9.82 \pm 0.06$ & $9.10 \pm 0.18$ & $>0.74$ & $>-2.26$ & $\ldots$ & Yes & Yes-faint & No & No & $\ldots$ \\
\hline $044008.0+260525$ & IRAS $04370+2559$ & $7.96 \pm 0.05$ & $7.38 \pm 0.05$ & $6.93 \pm 0.05$ & $5.93 \pm 0.05$ & $2.43 \pm 0.04$ & $0.75 \pm 0.22$ & $>-1.78$ & Yes & Yes & Yes & Yes & Yes & $\ldots$ \\
\hline $044039.7+251906$ & J04403979+2519061 AB & $9.84 \pm 0.05$ & $9.68 \pm 0.06$ & $9.62 \pm 0.05$ & $9.57 \pm 0.05$ & $7.55 \pm 0.05$ & $>1.00$ & $>-2.43$ & & Yes & Yes-faint & No & No & . \\
\hline $044049.5+255119$ & JH223 & $8.90 \pm 0.05$ & $8.60 \pm 0.05$ & $8.24 \pm 0.05$ & $7.74 \pm 0.05$ & $5.13 \pm 0.04$ & $2.20 \pm 0.22$ & $>0.93$ & Yes & Yes & Yes & Yes & Yes & . \\
\hline $044104.2+255756$ & Haro 6-32 & $9.66 \pm 0.05$ & $9.56 \pm 0.06$ & $9.49 \pm 0.05$ & $9.46 \pm 0.06$ & $9.59 \pm 0.33$ & $>0.70$ & $>-0.83$ & $\ldots$ & No & No & No & No & \\
\hline $044104.7+245106$ & IW Tau AB & $8.13 \pm 0.05$ & $8.15 \pm 0.05$ & $8.08 \pm 0.05$ & $8.03 \pm 0.05$ & $7.97 \pm 0.07$ & $>1.08$ & $\ldots$ & $\ldots$ & No & No & No & No & .. \\
\hline $044108.2+255607$ & ITG $33 \mathrm{~A}$ & $9.68 \pm 0.05$ & $9.05 \pm 0.05$ & $8.49 \pm 0.05$ & $7.73 \pm 0.05$ & $4.60 \pm 0.04$ & $>0.67$ & $>0.73$ & $\ldots$ & Yes & Yes & Yes & Yes & . \\
\hline $044110.7+255511$ & ITG 34 & $10.78 \pm 0.05$ & $10.35 \pm 0.05$ & $9.92 \pm 0.06$ & $9.22 \pm 0.05$ & $6.48 \pm 0.05$ & $>0.74$ & $>-1.25$ & $\ldots$ & Yes & Yes & Yes & Yes & \\
\hline $044112.6+254635$ & IRAS04381+2540 & $9.15 \pm 0.05$ & $7.76 \pm 0.05$ & $6.72 \pm 0.05$ & $5.75 \pm 0.05$ & $1.43 \pm 0.04$ & $-1.92 \pm 0.22$ & $-4.33 \pm 0.34$ & Yes & Yes & Yes & Yes & Yes & \\
\hline $044138.8+255626$ & IRAS04385+2550 & $8.24 \pm 0.05$ & $7.74 \pm 0.05$ & $7.13 \pm 0.05$ & $6.05 \pm 0.05$ & $1.86 \pm 0.04$ & $-0.90 \pm 0.22$ & $-2.73 \pm 0.22$ & Yes & Yes & Yes & Yes & Yes & $\cdots$ \\
\hline $044148.2+253430$ & J04414825+2534304 & $11.43 \pm 0.05$ & $10.93 \pm 0.05$ & $10.50 \pm 0.06$ & $9.54 \pm 0.05$ & $6.33 \pm 0.05$ & $>1.02$ & $>-4.57$ & $\ldots$ & Yes & Yes-faint & Yes & Yes & \\
\hline $044205.4+252256$ & LkHa332/G2 AB & $7.99 \pm 0.05$ & $7.87 \pm 0.05$ & $7.74 \pm 0.05$ & $7.70 \pm 0.05$ & $7.18 \pm 0.05$ & $\ldots$ & $>-4.69$ & $\ldots$ & Yes & Yes & No & No & $\mathrm{b}$ \\
\hline $044207.3+252303$ & LkHa332/G1 AB & $7.65 \pm 0.05$ & $7.62 \pm 0.05$ & $7.53 \pm 0.05$ & $7.51 \pm 0.06$ & $\ldots$ & $>0.51$ & $>-2.34$ & $\ldots$ & $\ldots$ & $\ldots$ & No & No & $\mathrm{b}$ \\
\hline $044207.7+252311$ & V955 Tau Ab & $6.99 \pm 0.05$ & $6.58 \pm 0.05$ & $6.15 \pm 0.05$ & $5.40 \pm 0.05$ & $2.76 \pm 0.04$ & $-0.56 \pm 0.22$ & $>-2.07$ & Yes & Yes & Yes & Yes & Yes & $\mathrm{b}$ \\
\hline $044221.0+252034$ & CIDA-7 & $9.51 \pm 0.05$ & $9.11 \pm 0.05$ & $8.65 \pm 0.05$ & $7.79 \pm 0.05$ & $4.20 \pm 0.04$ & $1.13 \pm 0.22$ & $>-1.18$ & Yes & Yes & Yes & Yes & Yes & $\ldots$ \\
\hline $044237.6+251537$ & DP Tau & $7.57 \pm 0.05$ & $6.90 \pm 0.05$ & $6.34 \pm 0.05$ & $5.37 \pm 0.05$ & $1.90 \pm 0.04$ & $0.54 \pm 0.22$ & $>-1.70$ & Yes & Yes & Yes & Yes & Yes & $\ldots$ \\
\hline $044303.0+252018$ & GO Tau & $8.90 \pm 0.05$ & $8.64 \pm 0.05$ & $8.21 \pm 0.05$ & $7.42 \pm 0.05$ & $4.30 \pm 0.04$ & $1.03 \pm 0.22$ & $>0.53$ & Yes & Yes & Yes & Yes & Yes & $\ldots$ \\
\hline $044427.1+251216$ & IRAS04414+2506 & $9.56 \pm 0.05$ & $9.00 \pm 0.05$ & $8.36 \pm 0.05$ & $7.43 \pm 0.05$ & $4.25 \pm 0.04$ & $1.76 \pm 0.22$ & $>0.05$ & Yes & Yes & Yes & Yes & Yes & $\ldots$ \\
\hline $044642.6+245903$ & RXJ04467+2459 & $10.05 \pm 0.05$ & $9.97 \pm 0.05$ & $9.87 \pm 0.06$ & $9.90 \pm 0.05$ & $9.53 \pm 0.26$ & $>0.96$ & $\ldots$ & $\ldots$ & No & No & No & No & $\ldots$ \\
\hline
\end{tabular}

Notes.

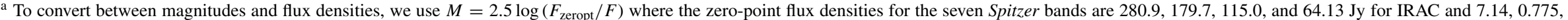
and $0.159 \mathrm{Jy}$ for MIPS. IRAC effective wavelengths are 3.6, 4.5, 5.8, and $8.0 \mu \mathrm{m}$; MIPS effective wavelengths are 24, 70, and $160 \mu \mathrm{m}$.

${ }^{\mathrm{b}}$ MIPS-160 flux density for this object is subject to confusion with a nearby source or sources.

${ }^{c}$ MIPS-160 flux density for this object is compromised by missing and/or saturated data.

${ }^{\mathrm{d}}$ MIPS-160 flux density for this object is hard saturated.

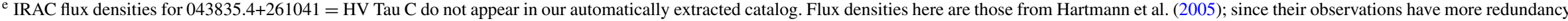
at IRAC bands, they are able to obtain reliable flux densities for this object at IRAC bands. MIPS flux densities are determined from our data.

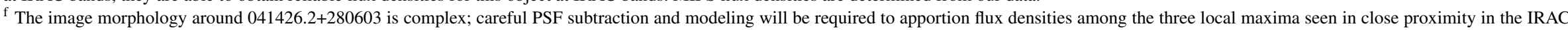
images, which may or may not be three physically distinct sources.

(This table is also available in a machine-readable form in the online journal.) 
Table 5

Spitzer Measurements for Sample of New Candidate Taurus Members ${ }^{\mathrm{a}}$

\begin{tabular}{|c|c|c|c|c|c|c|c|c|c|c|c|c|c|c|}
\hline SST Tau Name & Common Name & $\begin{array}{l}{[3.6]} \\
\text { (mag) }\end{array}$ & $\begin{array}{l}{[4.5]} \\
(\mathrm{mag})\end{array}$ & $\begin{array}{l}{[5.8]} \\
(\mathrm{mag})\end{array}$ & $\begin{array}{c}{[8]} \\
(\mathrm{mag})\end{array}$ & $\begin{array}{c}{[24]} \\
(\mathrm{mag})\end{array}$ & $\begin{array}{c}{[70]} \\
(\mathrm{mag})\end{array}$ & $\begin{array}{l}{[160]} \\
\text { (mag) }\end{array}$ & $\begin{array}{c}\text { Ident. in } \\
24 / 70 \\
\text { CMD }\end{array}$ & $\begin{array}{c}\text { Ident. in } \\
K_{\mathrm{S}} / 24 \\
\mathrm{CMD}\end{array}$ & $\begin{array}{c}\text { Ident. in } \\
8 / 24 \\
\text { CMD }\end{array}$ & $\begin{array}{c}\text { Ident. in } \\
4.5 / 8 \\
\text { CMD }\end{array}$ & $\begin{array}{l}\text { Ident. in } \\
\text { IRAC } \\
\text { CCD }\end{array}$ & Notes \\
\hline $041159.7+294236$ & 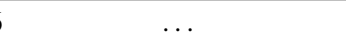 & & $10.91 \pm 0.05$ & & $10.22 \pm 0.06$ & $9.88 \pm 0.35$ & $>1.16$ & $>1.33$ & & Yes & No & Yes & & \\
\hline $041332.3+291726$ & , & $13.04 \pm 0.06$ & $12.96 \pm 0.06$ & $12.79 \pm 0.07$ & $11.96 \pm 0.07$ & $8.19 \pm 0.09$ & $2.45 \pm 0.22$ & $>0.08$ & No & Yes & No & No & No & .. \\
\hline $041339.4+292114$ & $\ldots$ & $12.80 \pm 0.06$ & $11.94 \pm 0.05$ & $11.15 \pm 0.06$ & $10.35 \pm 0.06$ & $7.54 \pm 0.06$ & $>1.20$ & $>1.46$ & . & No & No & Yes-faint & Yes & .. \\
\hline $041427.3+255130$ & $\ldots$ & $13.01 \pm 0.06$ & $12.95 \pm 0.06$ & $12.97 \pm 0.07$ & $12.22 \pm 0.08$ & $9.31 \pm 0.21$ & $>1.33$ & $>-0.25$ & & Yes & No & No & No & $\ldots$ \\
\hline $041535.6+284741$ & $\ldots$ & $13.20 \pm 0.06$ & $12.26 \pm 0.06$ & $11.33 \pm 0.06$ & $10.34 \pm 0.06$ & $5.65 \pm 0.04$ & $2.05 \pm 0.22$ & $>-1.53$ & Yes & No & Yes-faint & Yes-faint & Yes & 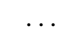 \\
\hline $041539.1+281858$ & $\ldots$ & $8.77 \pm 0.05$ & $8.52 \pm 0.05$ & $8.17 \pm 0.05$ & $7.50 \pm 0.05$ & $4.23 \pm 0.04$ & $1.08 \pm 0.22$ & $>-2.26$ & Yes & Yes & Yes & Yes & Yes & 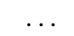 \\
\hline $041542.7+290959$ & $\ldots$ & $9.20 \pm 0.05$ & $9.18 \pm 0.05$ & $9.05 \pm 0.05$ & $9.00 \pm 0.06$ & $4.51 \pm 0.04$ & $-0.16 \pm 0.22$ & $>-2.01$ & Yes & Yes & Yes & No & No & 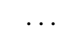 \\
\hline $041557.9+274617$ & $\ldots$ & $9.72 \pm 0.05$ & $9.40 \pm 0.05$ & $9.06 \pm 0.05$ & $8.37 \pm 0.05$ & $5.74 \pm 0.04$ & $>1.22$ & $>-1.58$ & . & Yes & Yes & Yes & Yes & $\ldots$ \\
\hline $041604.8+261801$ & $\ldots$ & $12.27 \pm 0.05$ & $11.50 \pm 0.05$ & $10.73 \pm 0.06$ & $59.85 \pm 0.06$ & $6.53 \pm 0.05$ & $>1.30$ & $>-1.27$ & & No & Yes-faint & Yes-faint & Yes & \\
\hline $041605.8+281426$ & . & $16.29 \pm 0.08$ & $15.41 \pm 0.09$ & $14.31 \pm 0.15$ & $13.27 \pm 0.12$ & $8.79 \pm 0.14$ & $2.36 \pm 0.22$ & $>-1.49$ & Yes-faint & & No & No & No & \\
\hline $041624.5+290858$ & HD 281820 & $9.49 \pm 0.05$ & $9.49 \pm 0.05$ & $9.45 \pm 0.05$ & $9.44 \pm 0.05$ & $8.28 \pm 0.09$ & $>1.25$ & $>-0.98$ & & Yes & Yes & No & No & \\
\hline $041706.2+264413$ & $\ldots$ & $11.08 \pm 0.05$ & $11.12 \pm 0.05$ & $11.10 \pm 0.06$ & $11.00 \pm 0.06$ & $8.92 \pm 0.16$ & $>0.96$ & $>0.18$ & & Yes & No & No & No & \\
\hline $041801.1+283526$ & $\ldots$ & $11.15 \pm 0.05$ & $11.12 \pm 0.05$ & $11.05 \pm 0.06$ & $10.94 \pm 0.06$ & $9.10 \pm 0.13$ & $>1.54$ & $>-0.22$ & 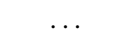 & Yes & No & No & No & . \\
\hline $041803.3+244009$ & $\ldots$ & $10.08 \pm 0.05$ & $10.07 \pm 0.05$ & $10.03 \pm 0.06$ & $9.83 \pm 0.05$ & $7.21 \pm 0.05$ & $>1.10$ & $>-0.39$ & .. & Yes & Yes-faint & No & No & . \\
\hline $041810.5+284447$ & $\ldots$ & $12.42 \pm 0.05$ & $11.73 \pm 0.05$ & $10.94 \pm 0.06$ & $10.06 \pm 0.06$ & $6.42 \pm 0.05$ & $1.84 \pm 0.22$ & $>2.04$ & Yes & No & No & Yes-faint & Yes & $\cdots$ \\
\hline $041810.7+251957$ & [GBM90] L1506 1 & $10 \pm 0.05$ & $7.76 \pm 0.05$ & $7.27 \pm 0.05$ & $6.33 \pm 0.05$ & $4.42 \pm 0.04$ & 1.72 & $>-1$ & Yes & Yes & Yes & Yes & res & $\mathrm{c}$ \\
\hline $041823.2+251928$ & 2MASX 04182321+2519281 & $11.79 \pm 0.05$ & $11.09 \pm 0.05$ & $10.44 \pm 0.06$ & $9.57 \pm 0.05$ & $6.18 \pm 0.05$ & $>1.18$ & $>-1.08$ & & Yes-faint & t Yes-faint & Yes-faint & Yes & $\cdots$ \\
\hline $041831.2+282617$ & $\ldots$ & $14.00 \pm 0.06$ & $13.24 \pm 0.06$ & $12.66 \pm 0.07$ & $11.82 \pm 0.06$ & $6.60 \pm 0.05$ & $2.40 \pm 0.22$ & $\ldots$ & Yes & $\cdots$ & Yes-faint & No & No & \\
\hline $041858.0+235031$ & $\ldots$ & $12.68 \pm 0.06$ & 11. & $11.07 \pm 0.06$ & $10.22 \pm 0.06$ & $7.27 \pm 0.05$ & $>1.20$ & $>0.33$ & & No & No & Yes-faint & Yes & . \\
\hline $041859.0+255740$ & $\ldots$ & $13.08 \pm 0.06$ & $12.06 \pm 0.06$ & $11.10 \pm 0.06$ & $5 \quad 9.90 \pm 0.06$ & $5.96 \pm 0.04$ & $>1.25$ & $>1.82$ & & No & Yes-faint & Yes-faint & Yes & $\ldots$ \\
\hline $041936.2+265256$ & $\ldots$ & $12.26 \pm 0.05$ & \pm 0.06 & $12.17 \pm 0.06$ & $12.09 \pm 0.06$ & $9.27 \pm 0.15$ & $>1.55$ & $>-0.29$ & .. & Yes & No & No & No & . \\
\hline $041940.4+270100$ & $\ldots$ & $12.81 \pm 0.06$ & $11.95 \pm 0.05$ & $11.15 \pm 0.06$ & $10.25 \pm 0.06$ & $6.69 \pm 0.05$ & $>1.63$ & $>0.34$ & & No & No & Yes-faint & Yes & \\
\hline $041941.4+271607$ & HH390 star & $11.25 \pm 0.05$ & $10.42 \pm 0.05$ & $9.87 \pm 0.05$ & $9.32 \pm 0.05$ & $3.23 \pm 0.04$ & $-0.79 \pm 0.22$ & $-3.01 \pm 0.22$ & Yes & Yes & Yes & Yes & Yes & $\ldots$ \\
\hline $041946.5+271255$ & $\ldots$ & $10.17 \pm 0.05$ & $9.47 \pm 0.05$ & $8.75 \pm 0.05$ & $8.14 \pm 0.05$ & $4.05 \pm 0.04$ & $>1.16$ & $>-1.12$ & & Yes & Yes & Yes & Yes & $\ldots$ \\
\hline $042016.1+282132$ & $\ldots$ & $11.86 \pm 0.05$ & $11.48 \pm 0.05$ & $11.13 \pm 0.06$ & $10.57 \pm 0.06$ & $8.23 \pm 0.08$ & $>1.07$ & $>-0.28$ & $\ldots$ & Yes & No & Yes-faint & Yes & .. \\
\hline $042021.4+281349$ & $\ldots$ & $12.82 \pm 0$ & $12.42 \pm$ & $12.13 \pm$ & $11.64 \pm$ & $8.86 \pm 0.12$ & $2.54 \pm 0.22$ & -0 & Yes-faint & No & No & No & Tes & .. \\
\hline $042025.8+281923$ & $\ldots$ & $9.19 \pm 0.05$ & $8.52 \pm 0.05$ & $7.92 \pm 0.05$ & $7.12 \pm$ & $3.78 \pm 0.04$ & $1.92=$ & $>-1.07$ & Yes & Yes & Yes & Yes & es & $\cdots$ \\
\hline $5.8+281641$ & $\ldots$ & $45 \pm 0.05$ & $9.39 \pm 0.05$ & $9.31 \pm$ & $9.31 \pm$ & $1 \pm$ & $>1.13$ & $>-$ & & No & $\mathrm{Y}$ & No & No & . \\
\hline $042026.0+280408$ & $\ldots$ & $.40 \pm 0.05$ & $9.03 \pm 0.05$ & $8.61 \pm 0.05$ & $7.02 \pm 0$ & $3.26 \pm 0.04$ & $0.85 \pm 0.22$ & $>0.42$ & Yes & Yes & Yes & Yes & Yes & $\ldots$ \\
\hline $042109.3+275036$ & $\ldots$ & $9.96 \pm 0.05$ & $9.78 \pm 0.05$ & $9.60 \pm 0.05$ & $9.22 \pm 0.05$ & $7.17 \pm 0.05$ & $>1.53$ & $>-1.65$ & $\ldots$ & Yes & Yes & Yes & Yes & $\ldots$ \\
\hline $042110.9+255259$ & V412 Tau & $<6.62$ & $<6.10$ & $5.37 \pm 0.05$ & $5.17 \pm 0.05$ & $4.33 \pm 0.04$ & $>1$ & $>0$ & $\ldots$ & Yes & Yes & & $\ldots$ & $\ldots$ \\
\hline $042135.6+253835$ & $\ldots$ & $12.22 \pm 0.05$ & $12.18 \pm 0.06$ & $12.15 \pm 0.06$ & $11.50 \pm 0.06$ & $9.44 \pm 0.25$ & $>1$ & $>-0.28$ & $\ldots$ & Yes & No & No & No & $\ldots$ \\
\hline $042146.3+242505$ & $\ldots$ & $7.73 \pm 0.05$ & $7.80 \pm 0.05$ & $7.57 \pm 0.05$ & $7.43 \pm 0.05$ & $6.71 \pm 0.05$ & $>1.19$ & $>1.12$ & $\cdots$ & Yes & Yes & No & No & $\cdots$ \\
\hline $042151.3+265720$ & $\ldots$ & $12.80 \pm 0.06$ & $12.63 \pm 0.06$ & $12.50 \pm 0.07$ & $11.79 \pm 0.21$ & $>11.00$ & $>1.49$ & $>-0.85$ & 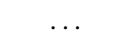 & 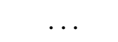 & $\ldots$ & No & Yes & \\
\hline $042200.9+235430$ & $\ldots$ & $7.74 \pm 0.05$ & $7.85 \pm 0.05$ & $7.66 \pm 0.05$ & $7.53 \pm 0.05$ & $7.01 \pm 0.05$ & $>1.24$ & $>0.68$ & & No & Yes & No & No & \\
\hline $042212.9+254659$ & $\ldots$ & $12.58 \pm 0.05$ & $11.84 \pm 0.05$ & $11.20 \pm 0.06$ & $10.36 \pm 0.06$ & $7.24 \pm 0.05$ & $>1.21$ & $>-0.14$ & $\cdots$ & No & No & Yes-faint & Yes & . \\
\hline $042215.6+265706$ & 2MASS J04221568+2657060 & $11.12 \pm 0.05$ & $10.55 \pm 0.05$ & $10.20 \pm 0.06$ & $9.75 \pm 0.05$ & $3.81 \pm 0.04$ & $-0.51 \pm 0.22$ & $>-1.95$ & Yes & Yes & Yes-faint & Yes & Yes & \\
\hline $042220.9+264248$ & & $13.63 \pm 0.06$ & $12.59 \pm 0.06$ & $11.64 \pm 0.06$ & $10.56 \pm 0.06$ & $6.95 \pm 0.05$ & $>1.51$ & $>-3.74$ & $\ldots$ & No & No & Yes-faint & No & \\
\hline $042247.8+264553$ & IRAS04196+2638 & $8.07 \pm 0.05$ & $7.55 \pm 0.05$ & $7.14 \pm 0.05$ & $6.28 \pm 0.05$ & $3.23 \pm 0.04$ & $0.96 \pm 0.22$ & $>-0.49$ & Yes & Yes & Yes & Yes & Yes & $\mathrm{bd}$ \\
\hline $042254.6+282354$ & NSV 1577 & $7.39 \pm 0.05$ & $7.38 \pm 0.05$ & $7.32 \pm 0.05$ & $7.23 \pm 0.05$ & $4.81 \pm 0.04$ & $0.96 \pm 0.22$ & $>-1.02$ & Yes & Yes & Yes & No & No & \\
\hline $042306.0+280119$ & J04230607+2801194 & $10.62 \pm 0.05$ & $10.31 \pm 0.05$ & $9.94 \pm 0.06$ & $9.34 \pm 0.05$ & $6.43 \pm 0.05$ & $>0.64$ & $>0.31$ & & Yes & Yes & Yes & Yes & $\mathrm{d}$ \\
\hline $042318.2+264115$ & J04231822+2641156 & $9.47 \pm 0.05$ & $9.15 \pm 0.05$ & $8.81 \pm 0.05$ & $8.10 \pm 0.05$ & $4.56 \pm 0.04$ & $1.82 \pm 0.22$ & $>-1.38$ & Yes & Yes & Yes & Yes & Yes & $\mathrm{d}$ \\
\hline $042325.9+250354$ & $\ldots$ & $10.01 \pm 0.05$ & $9.91 \pm 0.05$ & $9.64 \pm 0.05$ & $9.61 \pm 0.05$ & $9.42 \pm 0.20$ & $>1.19$ & $>-1.06$ & $\ldots$ & Yes & No & No & No & \\
\hline $042335.3+250302$ & FU Tau & $8.40 \pm 0.05$ & $7.92 \pm 0.05$ & $7.36 \pm 0.05$ & $6.71 \pm 0.05$ & $4.60 \pm 0.04$ & $2.39 \pm 0.22$ & $>-1.56$ & Yes & Yes & Yes & Yes & Yes & $\mathrm{d}$ \\
\hline
\end{tabular}


Table 5

(Continued)

\begin{tabular}{|c|c|c|c|c|c|c|c|c|c|c|c|c|c|c|}
\hline SST Tau Name & Common Name & $\begin{array}{l}{[3.6]} \\
\text { (mag) }\end{array}$ & $\begin{array}{l}{[4.5]} \\
(\mathrm{mag})\end{array}$ & $\begin{array}{l}{[5.8]} \\
\text { (mag) }\end{array}$ & $\begin{array}{c}{[8]} \\
(\mathrm{mag})\end{array}$ & $\begin{array}{c}{[24]} \\
(\mathrm{mag})\end{array}$ & $\begin{array}{c}{[70]} \\
(\mathrm{mag})\end{array}$ & $\begin{array}{l}{[160]} \\
\text { (mag) }\end{array}$ & $\begin{array}{c}\text { Ident. in } \\
24 / 70 \\
\text { CMD }\end{array}$ & $\begin{array}{c}\text { Ident. in } \\
K_{\mathrm{S}} / 24 \\
\mathrm{CMD}\end{array}$ & $\begin{array}{c}\text { Ident. in } \\
8 / 24 \\
\text { CMD }\end{array}$ & $\begin{array}{c}\text { Ident. in } \\
4.5 / 8 \\
\text { CMD }\end{array}$ & $\begin{array}{l}\text { Ident. in } \\
\text { IRAC } \\
\text { CCD }\end{array}$ & \\
\hline $042339.0+251855$ & & $15.86 \pm 0.08$ & $15.60 \pm 0.08$ & $14.67 \pm 0.22$ & $13.59 \pm 0.15$ & $8.59 \pm 0.09$ & $2.47 \pm 0.22$ & $>-0.94$ & Yes-faint & $\ldots$ & No & No & No & $\ldots$ \\
\hline $042350.1+264006$ & FS 116 & $10.69 \pm 0.05$ & $10.58 \pm 0.05$ & $10.47 \pm 0.06$ & $10.16 \pm 0.06$ & $>9.96$ & $>1.14$ & $>-1.04$ & $\ldots$ & . & $\ldots$ & Yes & No & $\ldots$ \\
\hline $042356.0+242705$ & HD 283663 & $9.42 \pm 0.05$ & $9.39 \pm 0.05$ & $9.34 \pm 0.05$ & $9.32 \pm 0.05$ & $8.30 \pm 0.09$ & $>1.09$ & $>-0.71$ & . & Yes & Yes & No & No & $\ldots$ \\
\hline $042358.6+244742$ & & $10.91 \pm 0.05$ & $10.88 \pm 0.05$ & $10.86 \pm 0.06$ & $10.24 \pm 0.06$ & $>9.92$ & $>1.28$ & $>0.82$ & $\ldots$ & $\ldots$ & $\ldots$ & Yes & No & $\ldots$ \\
\hline $042420.9+263051$ & J04242090+2630511 & $11.83 \pm 0.05$ & $11.45 \pm 0.05$ & $11.00 \pm 0.06$ & $10.40 \pm 0.06$ & $7.46 \pm 0.06$ & $>1.13$ & $>-3.45$ & . & Yes & No & Yes-faint & Yes & $\mathrm{d}$ \\
\hline $042423.2+265008$ & $\ldots$ & $9.46 \pm 0.05$ & $9.40 \pm 0.05$ & $9.32 \pm 0.05$ & $9.21 \pm 0.05$ & $7.87 \pm 0.07$ & $>0.82$ & $>-0.57$ & 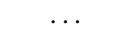 & Yes & Yes & No & No & 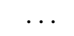 \\
\hline $042515.5+282927$ & $\ldots$ & $9.89 \pm 0.05$ & $9.79 \pm 0.05$ & $9.71 \pm 0.05$ & $9.71 \pm 0.05$ & $9.31 \pm 0.20$ & $>0.81$ & $>-0.24$ & . & Yes & No & No & No & . \\
\hline $042518.6+255535$ & . & $<6.62$ & $6.61 \pm 0.05$ & $6.39 \pm 0.05$ & $6.24 \pm 0.05$ & $5.62 \pm 0.04$ & $>1.05$ & $>0.28$ & - & Yes & Yes & No & $\ldots$ & $\mathrm{c}$ \\
\hline $042519.1+234716$ & HD 27923 & $7.64 \pm 0.05$ & $7.63 \pm 0.05$ & $7.64 \pm 0.05$ & $7.65 \pm 0.05$ & $7.01 \pm 0.05$ & $>1.03$ & $>-1.61$ & . & No & Yes & No & No & $\ldots$ \\
\hline $042558.8+273701$ & HD 283637 & $9.13 \pm 0.05$ & $9.11 \pm 0.05$ & $9.04 \pm 0.05$ & $9.06 \pm 0.05$ & $8.43 \pm 0.10$ & $>1.01$ & $>-2.68$ & & No & Yes & No & No & . \\
\hline $042653.3+255858$ & 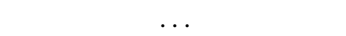 & $15.87 \pm 0.07$ & $15.25 \pm 0.08$ & $14.02 \pm 0.13$ & $12.28 \pm 0.08$ & $7.80 \pm 0.07$ & $1.57 \pm 0.22$ & $>0.21$ & Yes-faint & & No & No & No & \\
\hline $042721.0+240829$ & 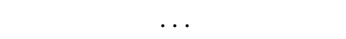 & $13.30 \pm 0.14$ & $13.08 \pm 0.06$ & $13.11 \pm 0.08$ & $12.64 \pm 0.09$ & $>9.71$ & $>1.00$ & $>0.59$ & & & & No & Yes & \\
\hline $042728.1+262323$ & . & $12.75 \pm 0.08$ & $12.58 \pm 0.06$ & $12.51 \pm 0.06$ & $11.83 \pm 0.20$ & $10.17 \pm 0.26$ & $>0.92$ & $>-2.33$ & & Yes & No & No & Yes & \\
\hline $042730.2+244123$ & 2MASX $04273023+2441232$ & $12.86 \pm 0.07$ & $12.87 \pm 0.07$ & $13.06 \pm 0.14$ & $14.34 \pm 2.95$ & $6.68 \pm 0.05$ & $0.41 \pm 0.22$ & $>-1.55$ & Yes & Yes & Yes-faint & No & No & $\ldots$ \\
\hline $042810.4+243553$ & $\ldots$ & $13.55 \pm 0.06$ & $12.53 \pm 0.06$ & $11.63 \pm 0.06$ & $10.54 \pm 0.08$ & $7.49 \pm 0.06$ & $>1.05$ & & $\ldots$ & $\cdots$ & No & Yes-faint & No & $\ldots$ \\
\hline $042857.4+243607$ & $\ldots$ & $10.40 \pm 0.05$ & $10.38 \pm 0.05$ & $10.25 \pm 0.06$ & $10.20 \pm 0.06$ & $8.88 \pm 0.14$ & $>1.10$ & $>1.85$ & . & Yes & No & No & No & $\cdots$ \\
\hline $042902.9+243140$ & 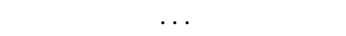 & $7.78 \pm 0.05$ & $7.68 \pm 0.05$ & $7.50 \pm 0.05$ & $7.46 \pm 0.05$ & $7.40 \pm 0.06$ & $>1$ & $>-1.56$ & $\ldots$ & Yes & No & No & No & $\cdots$ \\
\hline $042905.2+261535$ & 2MASX 04290517+2615358 & $13.30 \pm 0.06$ & $13.14 \pm 0.06$ & $13.15 \pm 0.08$ & $12.62 \pm 0.09$ & $>11.04$ & $>1.15$ & $>-1.27$ & $\ldots$ & $\ldots$ & $\ldots$ & No & Yes & $\cdots$ \\
\hline $042916.2+285627$ & HD 283629 & $9.20 \pm 0.05$ & $9.19 \pm 0.05$ & $9.16 \pm 0.06$ & $9.11 \pm 0.05$ & $8.55 \pm 0.11$ & $>1.09$ & ... & . & No & Yes & No & No & \\
\hline $042920.8+274207$ & IRAS $04262+2735$ & $6.67 \pm 0.05$ & $6.59 \pm 0.05$ & $6.22 \pm 0.05$ & $5.04 \pm 0.05$ & $3.02 \pm 0.04$ & $>1.01$ & $>-0.85$ & . & Yes & Yes & Yes & No & $\mathrm{b}$ \\
\hline $042932.0+243059$ & $\ldots$ & $10.41 \pm 0.05$ & $9.27 \pm 0.05$ & $8.32 \pm 0.05$ & $7.32 \pm 0.05$ & $3.91 \pm 0.04$ & $1.19 \pm 0.22$ & $>-1.43$ & Yes & Yes-faint & Yes & Yes & Yes & $\ldots$ \\
\hline $042936.0+243555$ & J04293606+2435556 & $8.04 \pm 0.05$ & $7.72 \pm 0.05$ & $7.47 \pm 0.05$ & $6.98 \pm 0.05$ & $4.19 \pm 0.04$ & $1.21 \pm 0.22$ & $>-1.75$ & Yes & Yes & Yes & Yes & Yes & $\mathrm{d}$ \\
\hline $042949.9+284253$ & $\ldots$ & $13.25 \pm 0.06$ & $12.57 \pm 0.06$ & $11.82 \pm 0.06$ & $10.79 \pm 0.06$ & $7.46 \pm 0.06$ & $>1.12$ & $>-0.70$ & & No & No & No & Yes & $\ldots$ \\
\hline $043004.7+283306$ & $\ldots$ & $9.47 \pm 0.05$ & $9.51 \pm 0.05$ & $9.48 \pm 0.05$ & $9.37 \pm 0.05$ & $8.63 \pm 0.12$ & $>0.95$ & $>-2.55$ & - & No & Yes & No & No & $\ldots$ \\
\hline $043024.1+281916$ & $\ldots$ & $6.92 \pm 0.05$ & $6.92 \pm 0.05$ & $6.75 \pm 0.05$ & $6.55 \pm 0.05$ & $5.84 \pm 0.04$ & $>1.06$ & $>-0.49$ & 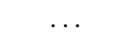 & Yes & Yes & No & No & $\mathrm{c}$ \\
\hline $043034.2+252427$ & $\ldots$ & $13.64 \pm 0.06$ & $13.56 \pm 0.06$ & $13.42 \pm 0.09$ & $13.38 \pm 0.13$ & $9.46 \pm 0.27$ & $>0$ & $>-0.93$ & $\ldots$ & Yes-faint & No & No & No & $\ldots$ \\
\hline $043042.8+274329$ & $\ldots$ & $7.07 \pm 0.05$ & $7.06 \pm 0.05$ & $6.79 \pm 0.05$ & $6.74 \pm 0.05$ & $6.17 \pm 0.05$ & $>0.95$ & $>-1$ & $\ldots$ & Yes & Yes & No & No & $\mathrm{d}$ \\
\hline $043044.7+263308$ & $\ldots$ & $7.33 \pm 0.05$ & $7.35 \pm 0.05$ & $7.29 \pm 0.05$ & $7.21 \pm 0.05$ & $6.91 \pm 0.05$ & $2.29 \pm 0$ & $>-$ & Yes & No & No & No & No & $\ldots$ \\
\hline $.4+230025$ & $\ldots$ & $10.31 \pm 0.05$ & $10.34 \pm 0.05$ & $10.31 \pm 0.06$ & $9.92 \pm 0.06$ & $>9.41$ & $>0.99$ & $>-$ & $\ldots$ & $\ldots$ & $\ldots$ & Yes & No & $\ldots$ \\
\hline 043 & $\ldots$ & $9.56 \pm 0.05$ & $9.48 \pm 0.05$ & $9.49 \pm 0.05$ & $9.47 \pm 0.05$ & $8.82 \pm 0.14$ & $>0$ & $>1$ & $\ldots$ & No & Yes & No & No & $\ldots$ \\
\hline $1.2+293922$ & $\ldots$ & $12.76 \pm 0.06$ & $12.31 \pm 0.06$ & $12.65 \pm 0.07$ & $11.70 \pm 0.06$ & $>10.63$ & $>1$ & $>0$ & $\ldots$ & $\ldots$ & .. & No & Yes & $\ldots$ \\
\hline $043145.0+285908$ & $\ldots$ & $10.96 \pm 0.05$ & $10.96 \pm 0.05$ & $10.93 \pm 0.06$ & $10.81 \pm 0.06$ & $8.76 \pm 0.12$ & $>1$ & $>-0.70$ & $\ldots$ & Yes & No & No & No & $\ldots$ \\
\hline $043213.6+251746$ & GSC 01833-00754 & $9.65 \pm 0.05$ & $9.63 \pm 0.05$ & $9.59 \pm 0.05$ & $9.57 \pm 0.05$ & $8.95 \pm 0.15$ & $>1$ & $>-0.49$ & $\ldots$ & No & Yes-faint & No & No & $\ldots$ \\
\hline $043214.6+223742$ & $\ldots$ & $13.86 \pm 0.06$ & $12.70 \pm 0.06$ & $11.64 \pm 0.06$ & $10.52 \pm 0.06$ & $7.32 \pm 0.05$ & $>1$ & $>0.24$ & $\ldots$ & $\ldots$ & No & Yes-faint & No & $\ldots$ \\
\hline $043224.1+225108$ & $\ldots$ & $10.04 \pm 0.05$ & $9.69 \pm 0.05$ & $9.28 \pm 0.05$ & $8.49 \pm 0.05$ & $6.11 \pm 0.04$ & $>1$ & $>0.63$ & $\ldots$ & Yes & Yes & Yes & Yes & $\ldots$ \\
\hline $043225.1+264732$ & $\ldots$ & $11.08 \pm 0.05$ & $11.06 \pm 0.05$ & $11.01 \pm 0.06$ & $10.48 \pm 0.06$ & $10.18 \pm 0.27$ & $>1.00$ & $>-1.43$ & & Yes & No & Yes-faint & No & \\
\hline $043228.1+271122$ & $\ldots$ & $6.81 \pm 0.05$ & $6.79 \pm 0.05$ & $6.57 \pm 0.05$ & $6.42 \pm 0.05$ & $5.91 \pm 0.04$ & $>0.72$ & $>-0.59$ & & Yes & Yes & No & No & $\mathrm{c}$ \\
\hline $043244.2+230224$ & $\ldots$ & $14.10 \pm 0.06$ & $13.25 \pm 0.06$ & $12.12 \pm 0.06$ & $10.52 \pm 0.06$ & $6.63 \pm 0.05$ & $2.51 \pm 0.22$ & $>-0.12$ & Yes & No & No & No & No & $\ldots$ \\
\hline $043249.3+225308$ & $\ldots$ & $8.49 \pm 0.05$ & $8.18 \pm 0.05$ & $7.77 \pm 0.05$ & $7.05 \pm 0.06$ & & $\ldots$ & $>-0.86$ & $\ldots$ & $\ldots$ & $\ldots$ & Yes & Yes & $\ldots$ \\
\hline $043256.4+222342$ & HD 284481 & $9.06 \pm 0.05$ & $9.05 \pm 0.05$ & $9.01 \pm 0.05$ & $9.03 \pm 0.05$ & $8.45 \pm 0.11$ & $>1.03$ & $>-1.74$ & $\cdots$ & No & Yes & No & No & $\cdots$ \\
\hline $043304.2+292149$ & HD 282276 & $7.99 \pm 0.05$ & $7.92 \pm 0.05$ & $7.83 \pm 0.06$ & $7.17 \pm 0.06$ & $3.02 \pm 0.04$ & $>-0.35$ & $-4.73 \pm 0.34$ & $\ldots$ & Yes & Yes & Yes & No & $\cdots$ \\
\hline $043312.6+291250$ & HD 282277 & $8.73 \pm 0.05$ & $8.68 \pm 0.05$ & $8.70 \pm 0.05$ & $8.64 \pm 0.05$ & $7.98 \pm 0.08$ & $>0.95$ & $>0.29$ & $\cdots$ & No & Yes & No & No & $\cdots$ \\
\hline $043316.6+262724$ & & $13.16 \pm 0.06$ & $12.41 \pm 0.06$ & $11.72 \pm 0.06$ & $10.79 \pm 0.06$ & $7.02 \pm 0.03$ & $>1.16$ & $>-4.62$ & & No & No & No & Yes & \\
\hline $043326.2+224529$ & 2MASS J04332621+2245293 & $9.56 \pm 0.05$ & $9.46 \pm 0.05$ & $9.38 \pm 0.05$ & $9.33 \pm 0.05$ & $8.46 \pm 0.08$ & $1.94 \pm 0.22$ & $>-1.45$ & Yes-faint & Yes & Yes & No & No & \\
\hline
\end{tabular}


Table 5

\begin{tabular}{|c|c|c|c|c|c|c|c|c|c|c|c|c|c|c|}
\hline SST Tau Name & Common Name & $\begin{array}{l}{[3.6]} \\
\text { (mag) }\end{array}$ & $\begin{array}{l}{[4.5]} \\
(\mathrm{mag})\end{array}$ & $\begin{array}{l}{[5.8]} \\
(\mathrm{mag})\end{array}$ & $\begin{array}{c}{[8]} \\
(\mathrm{mag})\end{array}$ & $\begin{array}{c}{[24]} \\
(\mathrm{mag})\end{array}$ & $\begin{array}{c}{[70]} \\
(\mathrm{mag})\end{array}$ & $\begin{array}{l}{[160]} \\
\text { (mag) }\end{array}$ & $\begin{array}{l}\text { Ident. in } \\
24 / 70 \\
\text { CMD }\end{array}$ & $\begin{array}{c}\text { Ident. in } \\
K_{\mathrm{s}} / 24 \\
\mathrm{CMD} \\
\end{array}$ & $\begin{array}{c}\text { Ident. in } \\
8 / 24 \\
\text { CMD } \\
\end{array}$ & $\begin{array}{c}\text { Ident. in } \\
4.5 / 8 \\
\text { CMD }\end{array}$ & $\begin{array}{l}\text { Ident. in } \\
\text { IRAC } \\
\text { CCD }\end{array}$ & Notes \\
\hline $043339.0+222720$ & . & $9.97 \pm 0.05$ & $9.75 \pm 0.05$ & $9.55 \pm 0.05$ & $9.19 \pm 0.05$ & $4.85 \pm 0.04$ & $1.11 \pm 0.22$ & $>-0.43$ & Yes & Yes & Yes & Yes & Yes & $\ldots$ \\
\hline $043341.8+223836$ & . & $11.10 \pm 0.05$ & $11.01 \pm 0.05$ & $11.01 \pm 0.06$ & $10.51 \pm 0.06$ & $>9.86$ & $>1.08$ & $>-0.71$ & $\ldots$ & $\ldots$ & $\ldots$ & Yes-faint & No & $\ldots$ \\
\hline $043344.6+261500$ & & $8.79 \pm 0.05$ & $8.24 \pm 0.05$ & $7.80 \pm 0.05$ & $7.15 \pm 0.05$ & $4.56 \pm 0.04$ & $1.85 \pm 0.22$ & $>-1.48$ & Yes & Yes & Yes & Yes & Yes & $\mathrm{c}$ \\
\hline $043349.5+291528$ & IRAS 04306+2909 & $12.66 \pm 0.06$ & $11.45 \pm 0.05$ & $10.12 \pm 0.06$ & $8.62 \pm 0.05$ & $4.12 \pm 0.04$ & $0.60 \pm 0.22$ & $>-0.70$ & Yes & Yes-faint & Yes & Yes-faint & Yes & $\mathrm{b}$ \\
\hline $043352.4+261254$ & J04335245+2612548 & $13.24 \pm 0.06$ & $12.70 \pm 0.06$ & $12.24 \pm 0.06$ & $11.46 \pm 0.06$ & $8.44 \pm 0.11$ & $>1.07$ & $>-0.75$ & . & Yes-faint & No & No & Yes & $\mathrm{d}$ \\
\hline $043359.2+293636$ & & $7.16 \pm 0.05$ & $7.24 \pm 0.05$ & $7.05 \pm 0.05$ & $6.94 \pm 0.05$ & $6.38 \pm 0.05$ & $>0.84$ & $>-1.17$ & . & Yes & Yes & No & No & $\ldots$ \\
\hline $043419.5+265210$ & & $11.84 \pm 0.05$ & $11.76 \pm 0.05$ & $11.68 \pm 0.06$ & $11.46 \pm 0.06$ & $8.88 \pm 0.14$ & $>0.95$ & $>-0.60$ & . & Yes & No & No & No & $\ldots$ \\
\hline $043419.8+232649$ & HD 284530 & $8.22 \pm 0.05$ & $8.22 \pm 0.05$ & $8.18 \pm 0.05$ & $8.18 \pm 0.05$ & $7.46 \pm 0.06$ & $>0.67$ & $>-0.97$ & $\ldots$ & No & Yes & No & No & $\ldots$ \\
\hline $043435.4+264406$ & $\ldots$ & $<6.62$ & $6.64 \pm 0.05$ & $6.45 \pm 0.05$ & $6.22 \pm 0.05$ & $4.84 \pm 0.04$ & $>1.07$ & $>-0.91$ & $\ldots$ & Yes & Yes & Yes & $\ldots$ & $\mathrm{c}$ \\
\hline $043452.5+240244$ & . & $11.49 \pm 0.05$ & $11.46 \pm 0.05$ & $11.40 \pm 0.06$ & $11.05 \pm 0.06$ & $8.81 \pm 0.18$ & $>1.42$ & $>-1.43$ & $\ldots$ & Yes & No & Yes-faint & No & $\ldots$ \\
\hline $043456.9+225835$ & 2MASS J04345693+2258358 & $9.05 \pm 0.05$ & $9.06 \pm 0.05$ & $8.95 \pm 0.05$ & $8.93 \pm 0.05$ & $8.31 \pm 0.08$ & $>1.26$ & $>-0.89$ & . & No & Yes & No & No & 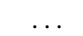 \\
\hline $043521.3+255510$ & & $13.24 \pm 0.06$ & $12.48 \pm 0.06$ & $11.71 \pm 0.06$ & $10.69 \pm 0.06$ & $7.27 \pm 0.06$ & $>1.08$ & $>-1.87$ & .. & No & No & No & Yes & $\ldots$ \\
\hline $043542.0+225222$ & 2MASS J04354203+2252226 & $9.69 \pm 0.05$ & $9.56 \pm 0.05$ & $9.50 \pm 0.05$ & $9.50 \pm 0.05$ & $8.98 \pm 0.12$ & $>1.07$ & $>-0.55$ & .. & Yes & Yes-faint & No & No & $\ldots$ \\
\hline $043557.6+225357$ & J04355760+2253574 & $13.06 \pm 0.05$ & $12.87 \pm 0.06$ & $12.43 \pm 0.06$ & $12.35 \pm 0.08$ & $7.13 \pm 0.05$ & $>0.88$ & $>-2.35$ & $\ldots$ & Yes-faint & Yes-faint & No & No & $\ldots$ \\
\hline $043559.4+223829$ & $\ldots$ & $12.41 \pm 0.06$ & $11.72 \pm 0.06$ & $10.87 \pm 0.06$ & $9.57 \pm 0.06$ & $5.22 \pm 0.04$ & $2.22 \pm 0.22$ & $>2.35$ & Yes & Yes & Yes-faint & Yes-faint & Yes & $\ldots$ \\
\hline $043621.4+271912$ & & $12.62 \pm 0.05$ & $12.62 \pm 0.06$ & $12.56 \pm 0.06$ & $12.38 \pm 0.07$ & $8.11 \pm 0.08$ & $>0.79$ & $>-0.87$ & .. & Yes & No & No & No & $\ldots$ \\
\hline $043621.5+235116$ & J04362151+2351165 & $11.78 \pm 0.05$ & $11.53 \pm 0.05$ & $11.12 \pm 0.06$ & $10.55 \pm 0.06$ & $7.74 \pm 0.07$ & $>1.06$ & $>-1.09$ & .. & Yes & No & Yes-faint & Yes & $\mathrm{d}$ \\
\hline $043636.1+265910$ & $\ldots$ & $11.59 \pm 0.05$ & $11.64 \pm 0.05$ & $11.61 \pm 0.06$ & $10.87 \pm 0.06$ & $>10.60$ & $>1.05$ & $>-1.20$ & $\ldots$ & $\ldots$ & $\ldots$ & Yes-faint & No & $\ldots$ \\
\hline $043642.0+265339$ & $\ldots$ & $12.93 \pm 0.06$ & $11.64 \pm 0.05$ & $10.23 \pm 0.06$ & $9.08 \pm 0.05$ & $6.18 \pm 0.05$ & $1.54 \pm 0.22$ & $>-1.93$ & Yes & $\ldots$ & Yes & Yes-faint & Yes & $\ldots$ \\
\hline $043720.8+250019$ & & $9.73 \pm 0.05$ & $9.72 \pm 0.06$ & $9.66 \pm 0.05$ & $9.63 \pm 0.05$ & $8.84 \pm 0.15$ & $>1.01$ & $>-0.23$ & $\ldots$ & No & Yes-faint & No & No & $\ldots$ \\
\hline $043724.8+270919$ & SV* SVS 1085 & $9.45 \pm 0.05$ & $9.30 \pm 0.05$ & $9.07 \pm 0.05$ & $8.73 \pm 0.05$ & $7.32 \pm 0.05$ & $>1.01$ & $>-1.38$ & $\ldots$ & Yes & Yes & Yes & No & $\ldots$ \\
\hline $043756.7+254622$ & ITG 1 & $12.03 \pm 0.05$ & $11.42 \pm 0.05$ & $10.73 \pm 0.06$ & $9.83 \pm 0.05$ & $7.16 \pm 0.05$ & $>0.67$ & $>-1.17$ & $\ldots$ & Yes & Yes-faint & Yes-faint & Yes & $\mathrm{bd}$ \\
\hline $043801.9+251926$ & $\ldots$ & $11.87 \pm 0.05$ & $11.76 \pm 0.05$ & $11.58 \pm 0.06$ & $10.98 \pm 0.06$ & $8.82 \pm 0.12$ & $>0.88$ & $>-2.12$ & $\ldots$ & Yes & No & Yes-faint & No & $\ldots$ \\
\hline $043803.6+221223$ & $\ldots$ & $11.46 \pm 0.05$ & $11.47 \pm 0.05$ & $11.46 \pm 0.06$ & $10.81 \pm 0.06$ & $10.85 \pm 0.33$ & $>1.09$ & $>0.43$ & $\ldots$ & No & No & Yes-faint & No & $\ldots$ \\
\hline $043816.5+261450$ & $\ldots$ & $9.45 \pm 0.05$ & $9.36 \pm 0.05$ & $9.17 \pm 0.05$ & $9.14 \pm 0.05$ & $9.00 \pm 0.14$ & $>0.67$ & $>-0.30$ & $\ldots$ & Yes & No & No & No & $\ldots$ \\
\hline $043826.7+265501$ & $\ldots$ & $9.80 \pm 0.05$ & $9.81 \pm 0.05$ & $9.77 \pm 0.05$ & $9.78 \pm 0.05$ & $9.05 \pm 0.15$ & $>1.01$ & $>1.08$ & $\ldots$ & No & Yes-faint & No & No & $\ldots$ \\
\hline $043905.2+233745$ & J04390525+2337450 & $10.60 \pm 0.06$ & $10.19 \pm 0.05$ & $9.75 \pm 0.05$ & $9.02 \pm 0.05$ & $3.51 \pm 0.04$ & $>0.25$ & $>-1.03$ & $\ldots$ & Yes & Yes & Yes & Yes & $\mathrm{d}$ \\
\hline $043933.6+235921$ & J04393364+2359212 & $9.63 \pm 0.05$ & $9.22 \pm 0.05$ & $8.85 \pm 0.05$ & $7.88 \pm 0.05$ & $5.22 \pm 0.04$ & $>0.92$ & $>-1.92$ & $\ldots$ & Yes & Yes & Yes & Yes & d \\
\hline $043939.9+252034$ & JH 225 & $8.60 \pm 0.05$ & $8.60 \pm 0.05$ & $8.50 \pm 0.05$ & $8.53 \pm 0.05$ & $7.66 \pm 0.06$ & $>0.74$ & $>-1.50$ & $\ldots$ & Yes & Yes & No & No & $\mathrm{c}$ \\
\hline $043943.8+271956$ & & $11.19 \pm 0.05$ & $11.25 \pm 0.05$ & $11.14 \pm 0.06$ & $10.81 \pm 0.06$ & $>9.99$ & $\ldots$ & $>0.47$ & $\ldots$ & $\ldots$ & $\ldots$ & Yes-faint & No & $\ldots$ \\
\hline $043944.8+260152$ & ITG 15 & $8.47 \pm 0.05$ & $8.13 \pm 0.05$ & $7.69 \pm 0.05$ & $7.04 \pm 0.05$ & $3.96 \pm 0.04$ & $1.32 \pm 0.22$ & $>-2.26$ & Yes & Yes & Yes & Yes & Yes & $\mathrm{bd}$ \\
\hline $044000.6+235821$ & J04400067+2358211 & $10.89 \pm 0.05$ & $10.64 \pm 0.05$ & $10.35 \pm 0.06$ & $9.70 \pm 0.06$ & $6.40 \pm 0.05$ & $>0.78$ & $>1.15$ & $\ldots$ & Yes & Yes-faint & Yes & Yes & $\mathrm{d}$ \\
\hline $044022.8+243307$ & $\ldots$ & $13.06 \pm 0.06$ & $12.25 \pm 0.06$ & $11.43 \pm 0.06$ & $10.46 \pm 0.06$ & $6.52 \pm 0.05$ & $>0.83$ & $>-0.91$ & $\ldots$ & No & No & Yes-faint & Yes & $\ldots$ \\
\hline $044023.0+255702$ & $\ldots$ & $10.68 \pm 0.05$ & $10.63 \pm 0.05$ & $10.57 \pm 0.06$ & $10.43 \pm 0.06$ & $9.36 \pm 0.22$ & $>0.92$ & $>-1.37$ & $\ldots$ & Yes & No & No & No & $\ldots$ \\
\hline $044048.4+233941$ & $\ldots$ & $13.52 \pm 0.06$ & $\ldots$ & $13.55 \pm 0.08$ & $\ldots$ & $9.35 \pm 0.20$ & $>1.12$ & $>-0.74$ & $\ldots$ & Yes-faint & $\ldots$ & $\ldots$ & $\ldots$ & $\ldots$ \\
\hline $044124.6+254353$ & ITG 40 & $10.43 \pm 0.05$ & $9.85 \pm 0.05$ & $9.39 \pm 0.05$ & $8.88 \pm 0.05$ & $5.64 \pm 0.04$ & $>0.95$ & $>-2.01$ & $\cdots$ & Yes & Yes & Yes & Yes & $\mathrm{bd}$ \\
\hline $044125.7+254349$ & $\ldots$ & $13.80 \pm 0.06$ & $12.41 \pm 0.06$ & $11.25 \pm 0.06$ & $10.22 \pm 0.06$ & $6.80 \pm 0.05$ & $2.32 \pm 0.22$ & $>-2.26$ & Yes & & No & Yes-faint & No & .. \\
\hline $044200.4+235813$ & $\ldots$ & $14.19 \pm 0.06$ & $13.04 \pm 0.06$ & $11.53 \pm 0.06$ & $9.94 \pm 0.05$ & $5.62 \pm 0.04$ & $1.49 \pm 0.22$ & $>-0.12$ & Yes & No & Yes-faint & Yes-faint & No & $\ldots$ \\
\hline $044241.1+244117$ & CCDM J04427+2441AB & $7.61 \pm 0.05$ & $7.55 \pm 0.05$ & $7.54 \pm 0.05$ & $7.52 \pm 0.05$ & $6.90 \pm 0.05$ & $>1.06$ & $>-1.45$ & $\ldots$ & No & Yes & No & No & $\ldots$ \\
\hline $044253.9+253709$ & $\ldots$ & $12.84 \pm 0.06$ & $12.79 \pm 0.06$ & $12.61 \pm 0.07$ & $12.48 \pm 0.08$ & $9.64 \pm 0.24$ & $>1.01$ & $>0.95$ & $\ldots$ & Yes & No & No & No & $\ldots$ \\
\hline $044315.8+235358$ & $\ldots$ & $12.94 \pm 0.06$ & $11.87 \pm 0.05$ & $10.78 \pm 0.06$ & $9.76 \pm 0.06$ & $6.49 \pm 0.05$ & $>0.95$ & $>-0.89$ & $\cdots$ & No & Yes-faint & Yes-faint & Yes & $\ldots$ \\
\hline $044325.1+255706$ & $\ldots$ & $9.35 \pm 0.05$ & $9.33 \pm 0.05$ & $9.20 \pm 0.05$ & $9.17 \pm 0.05$ & $8.59 \pm 0.12$ & $>0.87$ & $>-0.81$ & $\cdots$ & No & Yes & No & No & $\cdots$ \\
\hline $044345.3+243908$ & TYC 1834-591-1 & $9.49 \pm 0.05$ & $9.46 \pm 0.05$ & $9.42 \pm 0.05$ & $9.43 \pm 0.05$ & $8.31 \pm 0.09$ & $>1.04$ & $>0.07$ & $\ldots$ & Yes & Yes & No & No & $\cdots$ \\
\hline $044358.3+235103$ & $\ldots$ & $6.85 \pm 0.05$ & $6.73 \pm 0.05$ & $6.23 \pm 0.05$ & $6.19 \pm 0.05$ & $6.10 \pm 0.04$ & $>0.81$ & $>0.05$ & $\ldots$ & No & No & Yes & No & $\ldots$ \\
\hline
\end{tabular}


Table 5

(Continued)

\begin{tabular}{|c|c|c|c|c|c|c|c|c|c|c|c|c|c|c|}
\hline SST Tau Name & Common Name & $\begin{array}{l}{[3.6]} \\
\text { (mag) }\end{array}$ & $\begin{array}{l}{[4.5]} \\
(\mathrm{mag})\end{array}$ & $\begin{array}{l}{[5.8]} \\
\text { (mag) }\end{array}$ & $\begin{array}{c}{[8]} \\
(\mathrm{mag})\end{array}$ & $\begin{array}{c}{[24]} \\
(\mathrm{mag})\end{array}$ & $\begin{array}{c}{[70]} \\
(\mathrm{mag})\end{array}$ & $\begin{array}{l}{[160]} \\
(\mathrm{mag})\end{array}$ & $\begin{array}{c}\text { Ident. in } \\
24 / 70 \\
\text { CMD }\end{array}$ & $\begin{array}{c}\text { Ident. in } \\
K_{\mathrm{s}} / 24 \\
\mathrm{CMD}\end{array}$ & $\begin{array}{c}\text { Ident. in } \\
8 / 24 \\
\text { CMD }\end{array}$ & $\begin{array}{c}\text { Ident. in } \\
4.5 / 8 \\
\text { CMD }\end{array}$ & $\begin{array}{l}\text { Ident. in } \\
\text { IRAC } \\
\text { CCD }\end{array}$ & Notes \\
\hline $044518.2+242436$ & HD 30067 & $7.99 \pm 0.05$ & $8.02 \pm 0.05$ & $7.96 \pm 0.06$ & $7.95 \pm 0.05$ & $7.39 \pm 0.06$ & $>0.60$ & $>-0.65$ & $\cdots$ & No & Yes & No & No & . \\
\hline $044539.8+251704$ & $\ldots$ & $8.28 \pm 0.05$ & $8.33 \pm 0.05$ & $8.08 \pm 0.05$ & $8.00 \pm 0.05$ & $7.31 \pm 0.05$ & $>1.02$ & $>-0.35$ & $\ldots$ & Yes & Yes & No & No & $\mathrm{c}$ \\
\hline $044550.7+254448$ & $\ldots$ & $12.91 \pm 0.06$ & $11.94 \pm 0.05$ & $11.05 \pm 0.06$ & $10.09 \pm 0.06$ & $6.82 \pm 0.05$ & $>1.06$ & $>-1.01$ & $\ldots$ & No & No & Yes-faint & Yes & $\ldots$ \\
\hline $044555.7+261858$ & $\ldots$ & $10.99 \pm 0.05$ & $11.04 \pm 0.05$ & $10.91 \pm 0.06$ & $10.79 \pm 0.06$ & $9.00 \pm 0.16$ & $2.40 \pm 0.22$ & $>-0.43$ & Yes-faint & Yes & No & No & No & $\ldots$ \\
\hline $044557.0+244042$ & $\ldots$ & $13.12 \pm 0.06$ & $12.94 \pm 0.06$ & $12.62 \pm 0.07$ & $12.12 \pm 0.07$ & $>10.06$ & $>0.82$ & $>-0.13$ & $\ldots$ & $\ldots$ & $\ldots$ & No & Yes & $\ldots$ \\
\hline $044609.6+245237$ & $\ldots$ & $12.78 \pm 0.06$ & $12.69 \pm 0.06$ & $12.53 \pm 0.07$ & $12.43 \pm 0.07$ & $9.19 \pm 0.21$ & $>0.87$ & $>-1.49$ & $\ldots$ & Yes & No & No & No & $\ldots$ \\
\hline $044639.8+242526$ & $\ldots$ & $<6.62$ & $<6.10$ & $5.44 \pm 0.05$ & $5.27 \pm 0.05$ & $4.41 \pm 0.04$ & $>1.10$ & $>0.12$ & $\ldots$ & Yes & Yes & $\ldots$ & $\ldots$ & $\mathrm{c}$ \\
\hline $044644.4+262306$ & $\ldots$ & $<6.62$ & $6.17 \pm 0.05$ & $5.98 \pm 0.05$ & $5.82 \pm 0.05$ & $4.91 \pm 0.04$ & $>1.01$ & $>-3.17$ & $\ldots$ & Yes & Yes & No & $\ldots$ & $\ldots$ \\
\hline $044650.3+243815$ & $\ldots$ & $13.50 \pm 0.06$ & $13.11 \pm 0.22$ & $13.52 \pm 0.09$ & $13.22 \pm 0.11$ & $10.53 \pm 0.25$ & $>0.88$ & $>0.29$ & $\ldots$ & Yes-faint & No & No & Yes & $\ldots$ \\
\hline $044802.3+253359$ & $\ldots$ & $10.05 \pm 0.05$ & $10.06 \pm 0.05$ & $9.92 \pm 0.06$ & $9.90 \pm 0.06$ & $9.33 \pm 0.21$ & $>1.03$ & $>-1.34$ & $\ldots$ & No & Yes-faint & No & No & $\ldots$ \\
\hline $044832.3+234746$ & $\ldots$ & $12.89 \pm 0.06$ & $11.95 \pm 0.05$ & $11.05 \pm 0.06$ & $9.93 \pm 0.06$ & $6.30 \pm 0.05$ & $>0.92$ & $>-1.88$ & $\ldots$ & No & Yes-faint & Yes-faint & Yes & $\ldots$ \\
\hline $044857.4+255853$ & 2MASX $04485745+2558527$ & $13.30 \pm 0.06$ & $13.26 \pm 0.06$ & $12.94 \pm 0.08$ & $12.56 \pm 0.07$ & $9.60 \pm 0.25$ & $>1.00$ & $>-1.03$ & $\ldots$ & Yes-faint & No & No & No & $\ldots$ \\
\hline $044900.1+241346$ & $\ldots$ & $14.28 \pm 0.06$ & $13.82 \pm 0.06$ & $13.14 \pm 0.08$ & $10.41 \pm 0.06$ & $6.21 \pm 0.05$ & $1.78 \pm 0.22$ & $>-0.85$ & Yes & No & Yes-faint & No & No & $\ldots$ \\
\hline $044913.7+252549$ & $\ldots$ & $13.49 \pm 0.06$ & $12.81 \pm 0.18$ & $13.37 \pm 0.09$ & $11.91 \pm 0.06$ & $>9.24$ & $>0.83$ & $>-1.04$ & $\ldots$ & $\ldots$ & $\ldots$ & No & Yes & $\ldots$ \\
\hline $044916.3+243827$ & CCDM J04493+2438A & $8.92 \pm 0.05$ & $8.93 \pm 0.05$ & $8.87 \pm 0.05$ & $8.86 \pm 0.05$ & $8.35 \pm 0.09$ & $>0.88$ & $>-0.57$ & $\ldots$ & No & Yes & No & No & \\
\hline $044941.5+254010$ & . & $12.80 \pm 0.06$ & $12.73 \pm 0.06$ & $12.59 \pm 0.07$ & $12.61 \pm 0.09$ & $9.56 \pm 0.26$ & $>0.76$ & & $\cdots$ & Yes & No & No & No & $\cdots$ \\
\hline
\end{tabular}

Notes.

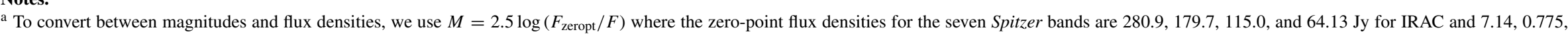
and $0.159 \mathrm{Jy}$ for MIPS. IRAC effective wavelengths are 3.6, 4.5, 5.8, and $8.0 \mu \mathrm{m}$; MIPS effective wavelengths are 24, 70, and $160 \mu \mathrm{m}$.

$\mathrm{b}$ Object previously identified in the literature as a potential YSO.

${ }^{c}$ Object previously identified in the literature as a potential non-member.

d Object identified as a Taurus member by other authors using portions of this Spitzer Legacy data set.

(This table is also available in a machine-readable form in the online journal.) 
Table 6

Derived Properties of Previously Identified Taurus Members

\begin{tabular}{|c|c|c|c|c|c|c|c|c|c|c|}
\hline SST Tau Name & Common Name & $\begin{array}{c}\mathrm{c} 2 \mathrm{~d} \\
\text { Category }\end{array}$ & $\begin{array}{c}\text { Gutermuth } \\
\text { Category }\end{array}$ & $\begin{array}{c}\text { Our } \\
\text { Class }\end{array}$ & Spectral Type & $\begin{array}{c}\text { Our H } \alpha \\
\text { EQW }(\AA)\end{array}$ & $\begin{array}{c}A_{V} \\
(\mathrm{mag})\end{array}$ & $\begin{array}{c}\log L_{*} \\
\left(L_{\odot}\right) \\
\end{array}$ & $L_{\mathrm{IR}} / L_{\text {total }}$ & Notes \\
\hline $041314.1+281910$ & LkCa 1 & YSO & Star & III & M4 & $\ldots$ & 1.3 & -0.3 & $<2.0 \mathrm{e}-05$ & $\ldots$ \\
\hline $041327.2+281624$ & Anon 1 & YSO & Star & III & M0 & $\ldots$ & 3.2 & 0.4 & $<3.8 \mathrm{e}-05$ & $\ldots$ \\
\hline $041353.2+281123$ & IRAS04108+2803 A & YSO & Class II & Flat & $\ldots$ & $\ldots$ & $\ldots$ & $\ldots$ & $\ldots$ & $\mathrm{c}$ \\
\hline $041354.7+281132$ & IRAS04108+2803 B & YSO & Embedded & I & $\ldots$ & $\ldots$ & $\ldots$ & $\ldots$ & $\ldots$ & $\mathrm{c}$ \\
\hline $041357.3+291819$ & IRAS04108+2910 & YSO & Class II & II & K5e & -201.4 & 4.8 & -0.4 & 0.37 & $a b$ \\
\hline $041411.8+281153$ & J04141188+2811535 & YSO & Class II & II & M6.25 & $\ldots$ & 1.7 & -1.6 & 0.25 & $\ldots$ \\
\hline $041412.2+280837$ & IRAS04111+2800G & YSO & Embedded & I & $\ldots$ & $\ldots$ & $\ldots$ & $\ldots$ & 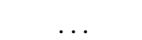 & $\mathrm{c}$ \\
\hline $041412.9+281212$ & V773 Tau ABC & $\ldots$ & $\ldots$ & II & K3 & $\ldots$ & 2.8 & 0.9 & 0.047 & $\ldots$ \\
\hline $041413.5+281249$ & FM Tau & YSO & Class II & II & M0 & -99.1 & 2.4 & -0.3 & 0.18 & $\mathrm{a}$ \\
\hline $041414.5+282758$ & FN Tau & YSO & Class II & II & M5 & $\ldots$ & 1.6 & -0.2 & 0.22 & $\ldots$ \\
\hline $041417.0+281057$ & CW Tau & $\ldots$ & $\ldots$ & II & K3 & -137.9 & 6.9 & 0.6 & 0.22 & $\mathrm{a}$ \\
\hline $041417.6+280609$ & CIDA-1 & YSO & Class II & II & M5.5 & $\ldots$ & 4.6 & -0.7 & 0.32 & $\ldots$ \\
\hline $041426.2+280603$ & IRAS04113+2758 A & $\ldots$ & $\ldots$ & I & M2.5 & $\ldots$ & 17.1 & 0.3 & 0.50 & $\ldots$ \\
\hline $041430.5+280514$ & MHO-3 & $\ldots$ & Embedded & Flat & K7 & $\ldots$ & 11.3 & 0.3 & 0.17 & $\ldots$ \\
\hline $041447.3+264626$ & FP Tau & YSO & Class II & II & M4 & $\ldots$ & 0.8 & -0.4 & 0.083 & $\ldots$ \\
\hline $041447.8+264811$ & CX Tau & YSO & Class II & II & M2.5 & $\ldots$ & 1.4 & -0.3 & 0.11 & $\ldots$ \\
\hline $041447.9+275234$ & $\mathrm{LkCa} 3 \mathrm{AB}$ & YSO & Star & III & M1 & $\ldots$ & 0.7 & 0.3 & $<4.3 \mathrm{e}-05$ & $\ldots$ \\
\hline $041449.2+281230$ & FO Tau AB & YSO & Class II & II & M2 & $\ldots$ & 3.7 & 0.0 & 0.094 & $\ldots$ \\
\hline $041505.1+280846$ & CIDA-2 & YSO & Star & III & M4.5 & $\ldots$ & 0.6 & -0.5 & $<8.2 \mathrm{e}-05$ & $\ldots$ \\
\hline $041514.7+280009$ & KPNO-1 & $\ldots$ & Star & III & M8.5 & $\ldots$ & 1.7 & -2.4 & 0.013 & $\ldots$ \\
\hline $041524.0+291043$ & J04152409+2910434 & $\ldots$ & Star & III & M7 & $\ldots$ & 1.3 & -1.9 & $<0.0046$ & $\ldots$ \\
\hline $041612.1+275638$ & $\mathrm{~J} 04161210+2756385$ & YSO & Class II & II & M4.75 & $\ldots$ & 4.6 & -0.9 & 0.15 & $\ldots$ \\
\hline $041618.8+275215$ & J04161885+2752155 & $\ldots$ & Star & III & M6.25 & $\ldots$ & 1.1 & -1.5 & $<0.0020$ & $\ldots$ \\
\hline $041628.1+280735$ & $\mathrm{LkCa} 4$ & YSO & Star & III & K7 & $\ldots$ & 0.8 & -0.0 & $<5.7 \mathrm{e}-05$ & $\ldots$ \\
\hline $041639.1+285849$ & J04163911+2858491 & YSO & Class II & II & M5.5 & $\ldots$ & 2.3 & -1.4 & 0.082 & $\ldots$ \\
\hline $041733.7+282046$ & CY Tau & YSO & Class II & II & M1 & $\ldots$ & 1.7 & -0.2 & 0.076 & $\ldots$ \\
\hline $041738.9+283300$ & LkCa 5 & $\ldots$ & $\ldots$ & III & M2 & $\ldots$ & 0.2 & -0.5 & $<5.7 \mathrm{e}-05$ & $\ldots$ \\
\hline $041749.5+281331$ & KPNO-10 & YSO & Class II & II & M5 & $\ldots$ & 1.1 & -1.2 & 0.12 & $\ldots$ \\
\hline $041749.6+282936$ & V410 X-ray 1 & YSO & Class II & II & M2 & $\cdots$ & 5.5 & -0.3 & 0.12 & $\cdots$ \\
\hline $041807.9+282603$ & V410 X-ray 3 & YSO & Star & III & M6 & $\ldots$ & 0.8 & -1.1 & $<0.00025$ & $\ldots$ \\
\hline $041817.1+282841$ & V410 Anon 13 & YSO & Class II & II & M5 & $\ldots$ & 5.9 & -1.1 & 0.068 & $\ldots$ \\
\hline $041822.3+282437$ & V410 Anon 24 & YSO & Star & II & G1 & $\ldots$ & 22.5 & 0.1 & $<5.8 \mathrm{e}-06$ & $\ldots$ \\
\hline $041829.0+282619$ & V410 Anon 25 & YSO & Star & II & M1 & $\ldots$ & 22.9 & 0.0 & $<1.2 \mathrm{e}-05$ & $\cdots$ \\
\hline $041830.3+274320$ & KPNO-11 & & Star & III & M5.5 & $\ldots$ & 0.0 & -1.3 & $<0.0018$ & $\ldots$ \\
\hline $041831.1+282716$ & V410 Tau ABC & YSO & Star & III & K3 & $\ldots$ & 1.1 & 0.4 & $<6.5 \mathrm{e}-05$ & $\ldots$ \\
\hline $041831.1+281629$ & DD Tau AB & $\ldots$ & $\ldots$ & II & M1 & $\ldots$ & 4.3 & 0.1 & 0.30 & $\ldots$ \\
\hline $041831.5+281658$ & CZ Tau AB & YSO & Embedded & II & M1.5 & $\ldots$ & 0.7 & -0.6 & 0.53 & $\ldots$ \\
\hline $041832.0+283115$ & IRAS04154+2823 & YSO & Class II & I & M2.5 & $\ldots$ & 19.0 & -0.6 & 0.63 & $\ldots$ \\
\hline $041834.4+283030$ & V410 X-ray 2 & YSO & Class II & Flat & M0 & $\ldots$ & 21.0 & 0.3 & 0.024 & $\ldots$ \\
\hline $041840.2+282424$ & V410 X-ray 4 & YSO & Star & II & M4 & $\ldots$ & 18.0 & -0.1 & $<6.3 \mathrm{e}-06$ & $\ldots$ \\
\hline $041840.6+281915$ & V892 Tau & $\ldots$ & $\ldots$ & Flat & A6 & -12.7 & 14.3 & 2.0 & 0.089 & $\ldots$ \\
\hline $041841.3+282725$ & LR1 & YSO & Class II & Flat & K4.5 & $\ldots$ & 24.0 & -0.3 & 0.12 & $\ldots$ \\
\hline $041842.5+281849$ & V410 X-ray 7 & YSO & Class II & II & M1 & $\ldots$ & 10.3 & -0.1 & 0.0092 & $\ldots$ \\
\hline $041845.0+282052$ & V410 Anon 20 & $\ldots$ & Star & Flat & $\mathrm{K} 3$ & $\ldots$ & 21.6 & -0.6 & $<0.00022$ & $\ldots$ \\
\hline $041847.0+282007$ & Hubble 4 & YSO & Star & III & K7 & $\ldots$ & 2.5 & 0.4 & $<6.0 \mathrm{e}-05$ & $\ldots$ \\
\hline $041851.1+281433$ & KPNO-2 & & Star & III & M7.5 & $\ldots$ & 0.3 & -2.1 & 0.0096 & $\ldots$ \\
\hline $041851.4+282026$ & CoKu Tau/1 & YSO & Embedded & I & K7 & $\ldots$ & 6.5 & -0.9 & 0.72 & $\ldots$ \\
\hline $041858.1+281223$ & IRAS04158+2805 & YSO & Class II & Flat & M6 & $\ldots$ & 7.0 & -1.3 & 0.76 & $\ldots$ \\
\hline $041901.1+281942$ & V410 X-ray 6 & YSO & Star & II & M4.5 & $\ldots$ & 2.7 & -0.5 & 0.082 & $\ldots$ \\
\hline $041901.2+280248$ & KPNO-12 & $\ldots$ & Class II & II & M9 & $\ldots$ & 1.4 & -2.9 & 0.12 & $\ldots$ \\
\hline $041901.9+282233$ & V410 Tau X-ray 5a & YSO & Star & III & M5 & $\ldots$ & 5.1 & -0.8 & $<0.00015$ & $\ldots$ \\
\hline $041912.8+282933$ & FQ Tau AB & YSO & Class II & II & M2 & $\cdots$ & 1.1 & -0.6 & 0.086 & $\cdots$ \\
\hline $041915.8+290626$ & BP Tau & YSO & Class II & II & K7 & $\ldots$ & 2.1 & 0.2 & 0.094 & $\ldots$ \\
\hline $041926.2+282614$ & V819 Tau & YSO & Star & III & K7 & $\ldots$ & 1.9 & 0.0 & 0.0018 & $\ldots$ \\
\hline $041935.4+282721$ & FR Tau & YSO & Class II & II & M5e & -58.9 & 0.0 & -0.9 & 0.17 & $\mathrm{~b}$ \\
\hline $041941.2+274948$ & $\mathrm{LkCa} 7 \mathrm{AB}$ & YSO & Star & III & K7 & $\ldots$ & 1.0 & 0.0 & $<6.1 \mathrm{e}-05$ & $\ldots$ \\
\hline $041942.5+271336$ & IRAS04166+2706 & YSO & Embedded & I & $\ldots$ & $\ldots$ & $\ldots$ & $\ldots$ & $\ldots$ & $\ldots$ \\
\hline $041958.4+270957$ & IRAS04169+2702 & YSO & Embedded & I & $\ldots$ & $\ldots$ & $\ldots$ & $\ldots$ & $\ldots$ & $\mathrm{c}$ \\
\hline $042025.5+270035$ & $\mathrm{~J} 04202555+2700355$ & YSO & Class II & II & M5.25 & $\ldots$ & 2.1 & -1.5 & 0.14 & $\ldots$ \\
\hline $042039.1+271731$ & 2MASS J04203918+2717317 & YSO & Star & III & $\ldots$ & $\ldots$ & $\ldots$ & $\ldots$ & $\ldots$ & $\ldots$ \\
\hline $042107.9+270220$ & CFHT-19 & YSO & Class II & I & M5.25 & $\ldots$ & 10.3 & -1.0 & 0.83 & $\ldots$ \\
\hline $042110.3+270137$ & IRAS04181+2654B & YSO & Class II & I & $\ldots$ & $\ldots$ & $\ldots$ & $\ldots$ & $\ldots$ & $\mathrm{c}$ \\
\hline $042111.4+270109$ & IRAS04181+2654A & YSO & Embedded & I & $\ldots$ & $\ldots$ & $\ldots$ & $\ldots$ & $\ldots$ & $\mathrm{c}$ \\
\hline $042134.5+270138$ & $\mathrm{~J} 04213459+2701388$ & YSO & Class II & II & M5.5 & $\cdots$ & 2.7 & -1.0 & 0.029 & $\ldots$ \\
\hline $042146.3+265929$ & CFHT- 10 & YSO & Class II & II & M6.25 & $\ldots$ & 4.0 & -1.7 & 0.056 & $\ldots$ \\
\hline
\end{tabular}


Table 6

(Continued)

\begin{tabular}{|c|c|c|c|c|c|c|c|c|c|c|}
\hline SST Tau Name & Common Name & $\begin{array}{c}\mathrm{c} 2 \mathrm{~d} \\
\text { Category }\end{array}$ & $\begin{array}{c}\text { Gutermuth } \\
\text { Category }\end{array}$ & $\begin{array}{c}\text { Our } \\
\text { Class } \\
\end{array}$ & Spectral Type & $\begin{array}{c}\text { Our H } \alpha \\
\text { EQW }(\AA)\end{array}$ & $\begin{array}{c}A_{V} \\
(\mathrm{mag})\end{array}$ & $\begin{array}{c}\log L_{*} \\
\left(L_{\odot}\right) \\
\end{array}$ & $L_{\mathrm{IR}} / L_{\text {total }}$ & Notes \\
\hline $042154.5+265231$ & J04215450+2652315 & Not YSO & Star & II & M8.5 & $\ldots$ & 3.3 & -2.4 & 0.012 & $\ldots$ \\
\hline $042155.6+275506$ & DE Tau & YSO & Class II & II & M2 & $\ldots$ & 2.2 & 0.1 & 0.13 & $\ldots$ \\
\hline $042157.4+282635$ & RY Tau & $\ldots$ & $\ldots$ & II & $\mathrm{K} 1$ & -16.8 & 4.8 & 1.3 & 0.10 & $\mathrm{a}$ \\
\hline $042158.8+281806$ & HD283572 & Star & Star & III & G5 & $\ldots$ & 0.4 & 0.8 & $<3.0 \mathrm{e}-05$ & $\ldots$ \\
\hline $042200.6+265732$ & FS Tau B & YSO & Embedded & I & $\ldots$ & $\ldots$ & $\ldots$ & $\ldots$ & $\ldots$ & $\mathrm{c}$ \\
\hline $042202.1+265730$ & FS Tau Aab & YSO & Class II & Flat & M0e & -46.8 & 6.9 & 0.0 & 0.36 & $a b$ \\
\hline $042203.1+282538$ & LkCa 21 & Star & Star & III & M3 & $\ldots$ & 1.3 & -0.2 & $<2.9 \mathrm{e}-05$ & $\ldots$ \\
\hline $042216.4+254911$ & CFHT-14 & & Star & III & M7.75 & $\ldots$ & 0.4 & -1.8 & $<0.0030$ & $\ldots$ \\
\hline $042216.7+265457$ & CFHT-21 & YSO & Class II & II & M1.25 & $\ldots$ & 7.7 & -0.3 & 0.23 & $\ldots$ \\
\hline $042224.0+264625$ & 2MASS J04222404+2646258 & YSO & Star & III & $\ldots$ & $\ldots$ & $\ldots$ & $\ldots$ & $\ldots$ & $\ldots$ \\
\hline $042307.7+280557$ & IRAS04200+2759 & YSO & Class II & Flat & M2 & $\ldots$ & 8.1 & -0.9 & 0.53 & $\mathrm{c}$ \\
\hline $042339.1+245614$ & FT Tau & YSO & Class II & II & M3e & -254.3 & 3.8 & -0.2 & 0.13 & $a b$ \\
\hline $042426.4+264950$ & CFHT-9 & YSO & Class II & II & M6.25 & $\ldots$ & 0.5 & -1.7 & 0.11 & $\ldots$ \\
\hline $042444.5+261014$ & IRAS04216+2603 & YSO & Class II & II & M1 & $\ldots$ & 3.4 & -0.4 & 0.29 & $\ldots$ \\
\hline $042445.0+270144$ & $\mathrm{~J} 1-4423$ & $\ldots$ & Star & III & M5 & $\ldots$ & 0.1 & -1.1 & $<0.00095$ & $\ldots$ \\
\hline $042449.0+264310$ & RXJ0424.8 & YSO & Star & III & K1 & $\ldots$ & 1.2 & 0.4 & $<6.1 \mathrm{e}-05$ & $\ldots$ \\
\hline $042457.0+271156$ & IP Tau & YSO & Class II & II & M0 & $\ldots$ & 1.9 & -0.2 & 0.10 & $\ldots$ \\
\hline $042517.6+261750$ & $\mathrm{~J} 1-4872 \mathrm{AB}$ & YSO & Star & III & K7 & $\ldots$ & 3.7 & 0.2 & $<2.6 \mathrm{e}-05$ & $\ldots$ \\
\hline $042629.3+262413$ & KPNO-3 & YSO & Class II & II & M6 & $\ldots$ & 1.6 & -1.7 & 0.13 & $\ldots$ \\
\hline $042630.5+244355$ & J04263055+2443558 & YSO & Class II & II & M8.75 & $\ldots$ & 0.6 & -2.4 & 0.16 & $\ldots$ \\
\hline $042653.5+260654$ & FV Tau AB & $\ldots$ & $\ldots$ & II & K5 & $\ldots$ & 8.7 & 0.6 & 0.13 & $\ldots$ \\
\hline $042654.4+260651$ & FV Tau/c AB & YSO & Class II & II & M3.5 & $\ldots$ & 5.9 & -0.2 & 0.096 & $\ldots$ \\
\hline $042656.2+244335$ & IRAS04239+2436 & $\ldots$ & Embedded & I & $\ldots$ & $\ldots$ & $\ldots$ & $\ldots$ & $\ldots$ & $\mathrm{c}$ \\
\hline $042657.3+260628$ & KPNO-13 & YSO & Class II & II & M5 & $\ldots$ & 4.3 & -0.6 & 0.078 & $\ldots$ \\
\hline $042702.6+260530$ & DG Tau B & $\ldots$ & $\ldots$ & I & $\ldots$ & $\ldots$ & $\ldots$ & $\ldots$ & $\ldots$ & $\ldots$ \\
\hline $042702.8+254222$ & DF Tau AB & $\ldots$ & $\ldots$ & II & M3 & $\ldots$ & 2.4 & 0.5 & 0.11 & $\ldots$ \\
\hline $042704.6+260616$ & DG Tau A & $\ldots$ & $\ldots$ & II & $\mathrm{K} 5 \mathrm{e}$ & -90.7 & 3.2 & 0.5 & 0.20 & $a b$ \\
\hline $042727.9+261205$ & KPNO-4 & Not YSO & Star & II & M9.5 & $\ldots$ & 2.8 & -2.3 & 0.037 & $\ldots$ \\
\hline $042745.3+235724$ & CFHT-15 & $\ldots$ & Star & III & M8.25 & $\ldots$ & 0.3 & -2.5 & $<0.0043$ & $\ldots$ \\
\hline $042757.3+261918$ & IRAS04248+2612 AB & YSO & Embedded & Flat & M5.5 & $\ldots$ & 5.8 & -0.6 & 0.55 & $\ldots$ \\
\hline $042838.9+265135$ & LDN 1521F-IRS & YSO & Contam. & I & $\ldots$ & $\ldots$ & $\ldots$ & $\ldots$ & $\ldots$ & $\ldots$ \\
\hline $042842.6+271403$ & $\mathrm{~J} 04284263+2714039 \mathrm{AB}$ & YSO & Class II & II & M5.25 & $\ldots$ & 3.7 & -1.0 & 0.050 & $\ldots$ \\
\hline $042900.6+275503$ & J04290068+2755033 & YSO & Class II & II & M8.25 & $\ldots$ & 0.3 & -2.1 & 0.11 & $\ldots$ \\
\hline $042904.9+264907$ & IRAS04260+2642 & YSO & Class II & I & K6 & $\ldots$ & 9.1 & -1.3 & 0.57 & $\ldots$ \\
\hline $042920.7+263340$ & $\mathrm{~J} 1-507$ & YSO & Star & III & M4 & $\ldots$ & 0.9 & -0.4 & $<4.3 \mathrm{e}-05$ & $\ldots$ \\
\hline $042921.6+270125$ & IRAS04263+2654 & YSO & Class II & II & M6.0 & $\ldots$ & 5.9 & -0.3 & 0.094 & $\ldots$ \\
\hline $042923.7+243300$ & GV Tau AB & & $\ldots$ & I & mid-K +emiss & -296.0 & 11.9 & 0.3 & $<0.92$ & $a b$ \\
\hline $042929.7+261653$ & FW Tau ABC & YSO & Star & III & M4 & $\ldots$ & 0.3 & -0.6 & 0.0019 & $\ldots$ \\
\hline $042930.0+243955$ & IRAS04264+2433 & YSO & Embedded & I & M1 & $\ldots$ & 9.5 & -0.9 & 0.75 & $\ldots$ \\
\hline $042941.5+263258$ & DH Tau AB & YSO & Star & II & M1 & $\ldots$ & 2.5 & -0.1 & 0.086 & $\ldots$ \\
\hline $042942.4+263249$ & DI Tau AB & $\ldots$ & Star & III & M0 & $\ldots$ & 0.5 & -0.1 & $<0.0011$ & $\ldots$ \\
\hline $042945.6+263046$ & KPNO-5 & $\ldots$ & Star & III & M7.5 & $\ldots$ & 0.6 & -1.6 & $<0.0025$ & $\ldots$ \\
\hline $042951.5+260644$ & IQ Tau & YSO & Class II & II & M0.5 & $\ldots$ & 2.9 & 0.1 & 0.19 & $\ldots$ \\
\hline $042959.5+243307$ & CFHT-20 & YSO & Class II & II & M5.5 & $\ldots$ & 4.6 & -0.7 & 0.10 & $\ldots$ \\
\hline $043007.2+260820$ & KPNO-6 & Not YSO & Class II & II & M8.5 & $\ldots$ & 1.2 & -2.4 & 0.10 & $\ldots$ \\
\hline $043023.6+235912$ & CFHT-16 & $\ldots$ & Star & III & M8.5 & $\ldots$ & 0.5 & -2.5 & $<0.0038$ & $\ldots$ \\
\hline $043029.6+242645$ & FX Tau AB & YSO & Class II & II & M1 & $\ldots$ & 2.9 & 0.1 & 0.081 & $\ldots$ \\
\hline $043044.2+260124$ & DK Tau AB & $\ldots$ & $\ldots$ & II & K7 & $\ldots$ & 2.9 & 0.4 & 0.13 & $\ldots$ \\
\hline $043050.2+230008$ & IRAS04278+2253 & & $\ldots$ & II & $\mathrm{F} 1$ & $\ldots$ & 13.6 & 1.8 & 0.075 & $\ldots$ \\
\hline $043051.3+244222$ & ZZ Tau AB & YSO & Class II & II & M5 & -9.7 & 1.6 & -0.2 & 0.029 & $\mathrm{~b}$ \\
\hline $043051.7+244147$ & ZZ Tau IRS & YSO & Class II & Flat & M4.5 & $\ldots$ & 6.9 & -0.9 & 0.76 & $\ldots$ \\
\hline $043057.1+255639$ & KPNO-7 & Not YSO & Class II & II & M8.25 & $\ldots$ & 0.3 & -2.3 & 0.13 & $\ldots$ \\
\hline $043114.4+271017$ & JH56 & YSO & Star & III & M0.5 & $\ldots$ & 0.0 & -0.3 & 0.0025 & $\ldots$ \\
\hline $043119.0+233504$ & J04311907+2335047 & $\ldots$ & Star & III & M7.75 & $\ldots$ & 1.2 & -1.8 & $<0.0032$ & $\ldots$ \\
\hline $043123.8+241052$ & V927 Tau AB & YSO & Star & III & M5.5 & $\ldots$ & 0.4 & -0.4 & $<5.5 \mathrm{e}-05$ & $\ldots$ \\
\hline $043126.6+270318$ & CFHT-13 & YSO & Star & III & M7.25 & $\ldots$ & 1.8 & -2.3 & 0.0062 & $\ldots$ \\
\hline $043150.5+242418$ & HK Tau AB & YSO & Class II & II & M0.5 & $\ldots$ & 4.7 & -0.2 & 0.22 & $\ldots$ \\
\hline $043158.4+254329$ & $\mathrm{~J} 1-665$ & YSO & Star & III & M5 & $\ldots$ & 1.2 & -0.6 & $<3.1 \mathrm{e}-05$ & $\ldots$ \\
\hline $043203.2+252807$ & J04320329+2528078 & $\ldots$ & Star & III & M6.25 & $\ldots$ & 0.0 & -1.2 & $<0.0025$ & $\ldots$ \\
\hline $043215.4+242859$ & Haro6-13 & $\ldots$ & $\ldots$ & Flat & M0 & $\ldots$ & 11.0 & 0.2 & 0.32 & $\ldots$ \\
\hline $043217.8+242214$ & CFHT-7 AB & YSO & Star & III & M6.5 & $\ldots$ & 0.9 & -1.1 & $<0.00021$ & $\ldots$ \\
\hline $043218.8+242227$ & V928 Tau AB & YSO & Star & III & M0.5 & $\ldots$ & 3.9 & 0.1 & $<2.9 \mathrm{e}-05$ & $\ldots$ \\
\hline $043223.2+240301$ & $\mathrm{~J} 04322329+2403013$ & $\ldots$ & Star & III & M7.75 & $\ldots$ & 0.0 & -1.5 & $<0.0026$ & $\ldots$ \\
\hline $043230.5+241957$ & FY Tau & YSO & Class II & II & K7 & $\ldots$ & 5.9 & 0.2 & 0.061 & $\ldots$ \\
\hline $043231.7+242002$ & FZ Tau & $\ldots$ & $\ldots$ & II & M0 & $\ldots$ & 7.2 & 0.4 & 0.17 & $\ldots$ \\
\hline
\end{tabular}


Table 6

(Continued)

\begin{tabular}{|c|c|c|c|c|c|c|c|c|c|c|}
\hline SST Tau Name & Common Name & $\begin{array}{c}\mathrm{c} 2 \mathrm{~d} \\
\text { Category }\end{array}$ & $\begin{array}{c}\text { Gutermuth } \\
\text { Category }\end{array}$ & $\begin{array}{c}\text { Our } \\
\text { Class } \\
\end{array}$ & Spectral Type & $\begin{array}{c}\text { Our H } \alpha \\
\text { EQW }(\AA)\end{array}$ & $\begin{array}{c}A_{V} \\
(\mathrm{mag}) \\
\end{array}$ & $\begin{array}{c}\log L_{*} \\
\left(L_{\odot}\right) \\
\end{array}$ & $L_{\mathrm{IR}} / L_{\text {total }}$ & Notes \\
\hline $043232.0+225726$ & IRAS04295+2251 & YSO & Embedded & I & $\ldots$ & $\ldots$ & $\ldots$ & $\ldots$ & $\ldots$ & $\mathrm{c}$ \\
\hline $043243.0+255231$ & UZ Tau Aab & & & II & M1 & -80.3 & 0.0 & -0.0 & 0.41 & $\mathrm{a}$ \\
\hline $043249.1+225302$ & JH112 & YSO & Class II & II & K6 & $\ldots$ & 5.5 & 0.1 & 0.11 & $\ldots$ \\
\hline $043250.2+242211$ & CFHT-5 & YSO & Star & III & M7.5 & $\ldots$ & 9.5 & -1.1 & $<0.00015$ & $\ldots$ \\
\hline $043301.9+242100$ & MHO-8 & YSO & Star & III & M5.5 & $\ldots$ & 0.9 & -0.8 & $<0.00012$ & $\ldots$ \\
\hline $043306.2+240933$ & GH Tau AB & YSO & Class II & II & M2 & $\ldots$ & 1.9 & 0.1 & 0.093 & $\ldots$ \\
\hline $043306.6+240954$ & V807 Tau AB & $\ldots$ & $\ldots$ & II & K7 & $\ldots$ & 1.3 & 0.5 & 0.050 & $\ldots$ \\
\hline $043307.8+261606$ & KPNO-14 & YSO & Star & III & M6 & $\ldots$ & 4.1 & -0.9 & $<0.00012$ & $\ldots$ \\
\hline $043309.4+224648$ & CFHT-12 & YSO & Class II & II & M6.5 & $\ldots$ & 3.2 & -1.5 & 0.036 & $\ldots$ \\
\hline $043310.0+243343$ & V830 Tau & YSO & Star & III & K7 & $\ldots$ & 0.7 & -0.1 & $<4.5 \mathrm{e}-05$ & $\ldots$ \\
\hline $043314.3+261423$ & IRAS04301+2608 & YSO & Contam. & Flat & M0 & $\ldots$ & 6.5 & -1.6 & 0.62 & $\mathrm{c}$ \\
\hline $043316.5+225320$ & IRAS04302+2247 & YSO & Star & I & $\ldots$ & $\ldots$ & $\ldots$ & $\ldots$ & $\ldots$ & $\mathrm{c}$ \\
\hline $043319.0+224634$ & IRAS04303+2240 & $\ldots$ & $\ldots$ & II & M0.5 & $\ldots$ & 10.8 & 0.2 & 0.45 & $\ldots$ \\
\hline $043334.0+242117$ & GI Tau & YSO & Class II & II & K6 & $\ldots$ & 2.7 & 0.2 & 0.19 & $\ldots$ \\
\hline $043334.5+242105$ & GK Tau & $\ldots$ & $\ldots$ & II & K7 & $\ldots$ & 2.7 & 0.3 & 0.16 & $\ldots$ \\
\hline $043336.7+260949$ & IS Tau AB & YSO & Class II & II & K7 & $\ldots$ & 3.5 & -0.2 & 0.15 & $\ldots$ \\
\hline $043339.0+252038$ & DL Tau & YSO & Class II & II & K7 & $\ldots$ & 2.8 & 0.0 & 0.26 & $\ldots$ \\
\hline $043342.9+252647$ & J04334291+2526470 & $\ldots$ & Star & III & M8.75 & $\ldots$ & 1.2 & -2.3 & $<0.0042$ & $\ldots$ \\
\hline $043352.0+225030$ & CI Tau & YSO & Class II & II & K7 & $\ldots$ & 3.6 & 0.2 & 0.14 & $\ldots$ \\
\hline $043352.5+225626$ & 2MASS J04335252+2256269 & YSO & Star & III & $\ldots$ & $\ldots$ & $\ldots$ & $\ldots$ & $\ldots$ & $\ldots$ \\
\hline $043354.7+261327$ & IT Tau AB & YSO & Class II & II & $\mathrm{K} 2$ & $\ldots$ & 13.4 & 0.9 & 0.0082 & $\ldots$ \\
\hline $043410.9+225144$ & JH108 & YSO & Star & III & M1 & $\ldots$ & 1.7 & -0.5 & $<6.8 \mathrm{e}-05$ & $\ldots$ \\
\hline $043415.2+225030$ & CFHT-1 & $\ldots$ & Star & II & $\ldots$ & $\ldots$ & $\ldots$ & $\ldots$ & $\ldots$ & $\ldots$ \\
\hline $043439.2+250101$ & Wa Tau 1 & Star & Star & III & K0 & $\ldots$ & 0.3 & 0.3 & $<3.4 \mathrm{e}-05$ & $\ldots$ \\
\hline $043455.4+242853$ & AA Tau & YSO & Class II & II & K7 & $\ldots$ & 2.2 & 0.1 & 0.15 & $\ldots$ \\
\hline $043508.5+231139$ & CFHT-11 & Not YSO & Star & III & M6.75 & $\ldots$ & 0.0 & -1.6 & 0.00051 & $\ldots$ \\
\hline $043520.2+223214$ & HO Tau & YSO & Class II & II & M0.5 & $\ldots$ & 2.6 & -0.7 & 0.11 & $\ldots$ \\
\hline $043520.8+225424$ & FF Tau AB & YSO & Star & III & K7 & 0.0 & 1.9 & -0.1 & $<6.3 \mathrm{e}-05$ & $\ldots$ \\
\hline $043527.3+241458$ & DN Tau & YSO & Class II & II & M0 & $\ldots$ & 1.1 & 0.0 & 0.095 & $\ldots$ \\
\hline $043535.3+240819$ & IRAS04325+2402 A & YSO & Class II & I & $\ldots$ & $\ldots$ & $\ldots$ & $\ldots$ & $\ldots$ & $\mathrm{c}$ \\
\hline $043540.9+241108$ & CoKu Tau/3 AB & YSO & Class II & II & M1 & $\ldots$ & 7.7 & 0.1 & 0.11 & $\ldots$ \\
\hline $043541.8+223411$ & KPNO-8 & $\ldots$ & Star & III & M5.75 & $\ldots$ & 0.0 & -1.7 & $<0.0020$ & $\ldots$ \\
\hline $043545.2+273713$ & $\mathrm{~J} 04354526+2737130$ & $\ldots$ & Star & III & M9.25 & $\ldots$ & 1.0 & -2.5 & $<0.0021$ & $\ldots$ \\
\hline $043547.3+225021$ & HQ Tau & $\ldots$ & $\ldots$ & II & $\mathrm{K} 2$ & -1.2 & 3.8 & 0.6 & 0.098 & $\mathrm{bc}$ \\
\hline $043551.0+225240$ & KPNO-15 & Star & Star & III & M1.5 & $\ldots$ & 2.7 & -0.7 & $<2.2 \mathrm{e}-05$ & $\ldots$ \\
\hline $043551.4+224911$ & KPNO-9 & $\ldots$ & Star & III & M8.5 & $\ldots$ & 1.4 & -2.6 & $<0.0040$ & $\ldots$ \\
\hline $043552.0+225503$ & 2MASS J04355209+2255039 & $\ldots$ & Star & III & $\ldots$ & $\ldots$ & $\ldots$ & $\ldots$ & $\ldots$ & $\ldots$ \\
\hline $043552.7+225423$ & HP Tau AB & $\ldots$ & $\ldots$ & II & K3 & -11.2 & 4.8 & 0.3 & 0.24 & $\mathrm{~d}$ \\
\hline $043552.8+225058$ & 2MASS J04355286+2250585 & YSO & Star & III & $\ldots$ & $\ldots$ & $\ldots$ & $\ldots$ & $\ldots$ & $\ldots$ \\
\hline $043553.4+225408$ & $\mathrm{HP} \mathrm{Tau} / \mathrm{G} 3 \mathrm{AB}$ & $\ldots$ & Star & III & K7 & $\ldots$ & 2.2 & -0.2 & $<0.0011$ & $\ldots$ \\
\hline $043554.1+225413$ & HP Tau/G2 & $\ldots$ & Star & III & G0 & $\ldots$ & 2.6 & 0.8 & $<0.00059$ & $\ldots$ \\
\hline $043556.8+225436$ & Haro 6-28 AB & YSO & Class II & II & M5 & $\ldots$ & 4.1 & -0.6 & 0.11 & $\ldots$ \\
\hline $043558.9+223835$ & 2MASS J04355892+2238353 & $\ldots$ & Star & III & $\ldots$ & $\ldots$ & $\ldots$ & $\ldots$ & $\ldots$ & $\ldots$ \\
\hline $043610.3+215936$ & $\mathrm{~J} 04361030+2159364$ & Not YSO & Class II & II & M8.5 & $\ldots$ & 0.8 & -2.4 & 0.096 & $\ldots$ \\
\hline $043610.3+225956$ & CFHT- 2 & $\ldots$ & Star & II & M7.5 & $\ldots$ & 3.0 & -1.8 & $<0.0024$ & $\ldots$ \\
\hline $043619.0+254258$ & $\mathrm{LkCa} 14$ & YSO & Star & III & M0 & $\ldots$ & 0.0 & -0.2 & $<4.5 \mathrm{e}-05$ & $\ldots$ \\
\hline $043638.9+225811$ & CFHT-3 & $\ldots$ & Star & III & M7.75 & $\ldots$ & 1.8 & -1.9 & $<0.0025$ & $\ldots$ \\
\hline $043649.1+241258$ & HD 283759 & YSO & Star & II & $\mathrm{F} 2$ & 5.5 & 0.4 & 0.4 & 0.0037 & $\mathrm{~b}$ \\
\hline $043800.8+255857$ & ITG 2 & YSO & Star & III & M7.25 & $\ldots$ & 2.3 & -0.9 & $<8.9 \mathrm{e}-05$ & $\ldots$ \\
\hline $043814.8+261139$ & J04381486+2611399 & YSO & Class II & I & M7.25 & $\ldots$ & 3.5 & -2.3 & 0.75 & $\ldots$ \\
\hline $043815.6+230227$ & RXJ0438.2+2302 & $\ldots$ & Star & III & M1 & $\ldots$ & 0.3 & -0.7 & $<0.00078$ & $\ldots$ \\
\hline $043821.3+260913$ & GM Tau & YSO & Class II & II & $\ldots$ & $\ldots$ & $\ldots$ & $\ldots$ & $\ldots$ & $\mathrm{c}$ \\
\hline $043828.5+261049$ & DO Tau & $\ldots$ & $\ldots$ & II & M0 & $\cdots$ & 4.9 & 0.3 & 0.28 & $\ldots$ \\
\hline $043835.2+261038$ & HV Tau AB & $\ldots$ & Star & III & M1 & $\ldots$ & 2.5 & 0.1 & $<0.0012$ & $\ldots$ \\
\hline $043835.4+261041$ & HV Tau C & YSO & Class II & Flat & $\mathrm{M} 4 \mathrm{e}$ & -10.0 & 1.9 & -1.7 & 0.71 & $\mathrm{~b}$ \\
\hline $043858.5+233635$ & J0438586+2336352 & $\ldots$ & $\ldots$ & II & M4.25 & $\ldots$ & 0.0 & -1.3 & 0.085 & $\ldots$ \\
\hline $043901.6+233602$ & $\mathrm{~J} 0439016+2336030$ & $\ldots$ & $\ldots$ & II & M6 & -3.3 & 0.9 & -1.0 & 0.044 & $\ldots$ \\
\hline $043903.9+254426$ & CFHT- 6 & YSO & Class II & II & M7.25 & $\ldots$ & 1.3 & -1.5 & 0.12 & $\ldots$ \\
\hline $043906.3+233417$ & J0439064+2334179 & $\ldots$ & $\ldots$ & III & M7.5 & $\ldots$ & 0.0 & -1.4 & $<0.0$ & $\ldots$ \\
\hline $043913.8+255320$ & IRAS04361+2547 AB & $\ldots$ & Class II & I & $\ldots$ & $\ldots$ & $\ldots$ & $\ldots$ & $\ldots$ & $\mathrm{c}$ \\
\hline $043917.7+222103$ & LkCa 15 & YSO & Class II & II & K5 & $\ldots$ & 1.9 & 0.0 & 0.10 & $\ldots$ \\
\hline $043920.9+254502$ & GN Tau B & YSO & Class II & II & M2.5 & $\ldots$ & 5.7 & 0.0 & 0.20 & $\ldots$ \\
\hline $043935.1+254144$ & IRAS04365+2535 & $\ldots$ & $\ldots$ & I & $\ldots$ & $\ldots$ & $\ldots$ & $\ldots$ & $\ldots$ & $\mathrm{c}$ \\
\hline $043947.4+260140$ & CFHT- 4 & YSO & Class II & II & M7 & $\ldots$ & 4.4 & -1.0 & 0.14 & $\ldots$ \\
\hline $043953.9+260309$ & IRAS $04368+2557$ & YSO & Embedded & I & $\ldots$ & $\ldots$ & $\ldots$ & $\ldots$ & $\ldots$ & $\ldots$ \\
\hline
\end{tabular}


Table 6

(Continued)

\begin{tabular}{|c|c|c|c|c|c|c|c|c|c|c|}
\hline SST Tau Name & Common Name & $\begin{array}{c}\mathrm{c} 2 \mathrm{~d} \\
\text { Category }\end{array}$ & $\begin{array}{c}\text { Gutermuth } \\
\text { Category }\end{array}$ & $\begin{array}{c}\text { Our } \\
\text { Class } \\
\end{array}$ & Spectral Type & $\begin{array}{c}\text { Our H } \alpha \\
\text { EQW }(\AA)\end{array}$ & $\begin{array}{c}A_{V} \\
(\mathrm{mag})\end{array}$ & $\begin{array}{c}\log L_{*} \\
\left(L_{\odot}\right) \\
\end{array}$ & $L_{\mathrm{IR}} / L_{\mathrm{total}}$ & Notes \\
\hline $043955.7+254502$ & IC2087 IRS & $\ldots$ & . & Flat & $\mathrm{K} 4$ & $\ldots$ & 18.0 & 1.3 & 0.41 & $\ldots$ \\
\hline $044001.7+255629$ & CFHT-17 AB & YSO & Star & III & M5.75 & $\ldots$ & 8.4 & -0.9 & $<0.00023$ & $\ldots$ \\
\hline $044008.0+260525$ & IRAS $04370+2559$ & YSO & Class II & II & $<\mathrm{M} 0$ & $\ldots$ & 13.1 & -0.0 & 0.15 & $\ldots$ \\
\hline $044039.7+251906$ & J04403979+2519061 AB & YSO & Star & III & M5.25 & $\ldots$ & 3.6 & -0.9 & 0.0042 & $\ldots$ \\
\hline $044049.5+255119$ & JH223 & YSO & Class II & II & M2 & $\ldots$ & 1.5 & -0.6 & 0.080 & $\ldots$ \\
\hline $044104.2+255756$ & Haro 6-32 & Star & Star & III & M5 & $\ldots$ & 0.6 & -0.9 & $<2.9 \mathrm{e}-05$ & $\ldots$ \\
\hline $044104.7+245106$ & IW Tau AB & Star & Star & III & K7 & $\ldots$ & 1.1 & 0.0 & $<4.0 \mathrm{e}-05$ & $\ldots$ \\
\hline $044108.2+255607$ & ITG $33 \mathrm{~A}$ & YSO & Class II & Flat & M3 & $\ldots$ & 8.3 & -1.1 & 0.33 & $\ldots$ \\
\hline $044110.7+255511$ & ITG 34 & YSO & Class II & II & M5.5 & $\ldots$ & 4.0 & -1.4 & 0.10 & $\ldots$ \\
\hline $044112.6+254635$ & IRAS04381+2540 & YSO & Embedded & I & $\ldots$ & $\ldots$ & $\ldots$ & $\ldots$ & $\ldots$ & $\mathrm{c}$ \\
\hline $044138.8+255626$ & IRAS04385+2550 & YSO & Class II & Flat & M0 & $\cdots$ & 9.3 & -0.2 & 0.30 & $\ldots$ \\
\hline $044148.2+253430$ & $\mathrm{~J} 04414825+2534304$ & YSO & Class II & II & M7.75 & $\ldots$ & 2.4 & -1.8 & 0.21 & $\ldots$ \\
\hline $044205.4+252256$ & LkHa332/G2 AB & YSO & Star & III & $\mathrm{K} 7$ & $\ldots$ & 4.3 & 0.1 & 0.00016 & $\ldots$ \\
\hline $044207.3+252303$ & LkHa332/G1 AB & $\ldots$ & Star & III & M1 & $\ldots$ & 4.6 & 0.2 & $<0.00065$ & $\ldots$ \\
\hline $044207.7+252311$ & V955 Tau Ab & YSO & Class II & II & K7 & $\ldots$ & 5.0 & 0.2 & 0.14 & $\ldots$ \\
\hline $044221.0+252034$ & CIDA-7 & YSO & Class II & II & M3 & $\ldots$ & 1.6 & -0.9 & 0.20 & $\ldots$ \\
\hline $044237.6+251537$ & DP Tau & YSO & Class II & II & M0.5 & $\cdots$ & 5.6 & -0.3 & 0.37 & $\ldots$ \\
\hline $044303.0+252018$ & GO Tau & YSO & Class II & II & M0 & $\cdots$ & 2.3 & -0.5 & 0.096 & $\cdots$ \\
\hline $044427.1+251216$ & IRAS04414+2506 & YSO & Class II & II & M7.25 & $\ldots$ & 1.6 & -1.3 & 0.44 & $\ldots$ \\
\hline $044642.6+245903$ & RXJ04467+2459 & YSO & Star & III & M4 & $\ldots$ & 0.0 & -1.0 & $<0.00015$ & $\ldots$ \\
\hline
\end{tabular}

Notes.

${ }^{a} \mathrm{Ca}$ IR triplet in emission in our spectrum.

b New spectral type, reported here.

${ }^{c}$ Unreduced IR spectrum in-hand, to be discussed in a later paper.

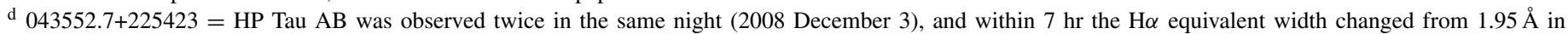
absorption to $11.24 \AA$ in emission.

(This table is also available in a machine-readable form in the online journal.)

was indicated as an upper limit. Note that this method is subject to several caveats: (a) objects that do not have spectral types (particularly common in the list of new candidate objects) do not have a calculated $A_{V}$, and therefore no $L_{*}$ or $L_{\mathrm{IR}} / L_{\text {total }}$; (b) this method of dereddening using $J-H$ ignores the potential for near-IR excess (as well as veiling and the H-opacity minimum at $1.6 \mu \mathrm{m}$ ) and therefore this method can overestimate $L_{*} ;$ (c) YSOs intrinsically vary at essentially all the wavelengths used here, and the photometry across the SED for each object was not obtained contemporaneously. Our values for $L_{\mathrm{IR}} / L_{\text {total }}$ are probably good to a factor of 2 , and are sufficient to determine if a source has an envelope, is an optically thick disk only, a highly flattened/thinning disk, or a debris-like system. (See Section 4.6 for more discussion.) This approach is demonstrably inaccurate in comparison to more complex methods, such as those employing bolometric corrections to reddening-corrected flux densities in well-calibrated passbands. However, more detailed modeling is beyond the scope of this paper.

For the 148 new candidate Taurus members, Table 7 also contains an adopted membership classification indicating whether or not we regard the object as a Taurus member. This classification combines information from all of the available photometric and optical spectroscopic data (see Sections 4.1 and 3.1.4). Extragalactic objects are noted as "xgal." Objects that we believe to be reliable new members are indicated as "new member." Objects that we think are likely to be new members but there is still some doubt are listed as "probable new member." Objects with the next gradation of confidence are "possible new members." Objects for which we have spectra but cannot determine clear membership are listed as "needs additional follow up" and, finally, objects with no spectroscopic data yet are "pending follow up." There are 34 new members, three probable new members,
10 possible new members, seven extragalactic objects, two other objects, 60 stars needing additional follow-up observations, and 33 pending any follow-up observations. Note that while all the "new members" are also grade A objects, not all grade A objects are "new members," because of the need for additional data in many cases.

A final data table, Table 8, lists the new members in order of quality, sorted by their category (new member, probable new member, possible new member, needs additional follow up, or pending follow up), and then by our rank (and then by catalog number), such that the objects we grade as most likely to be new members appear at the top of the list. Table 8 also contains the projected angular separation from to the nearest previously identified Taurus object.

\section{DISCUSSION}

\subsection{Overall Sample Properties}

\subsubsection{The Sample as a Whole}

There are 215 previously identified Taurus members and 148 new candidate Taurus members discussed here. Table 2 summarizes the samples selected from each color-magnitude or color-color space, along with relevant numbers from the SWIRE sample for comparison. Table 3 summarizes the fraction of the previously identified and new candidate sources detected at each Spitzer band.

Table 2 captures the fraction of objects found in each CMD or CCD that fall into the categories of previously identified YSOs, new candidate YSOs, previously identified non-members, and new non-members, and also the fraction of each of these categories that is found in each of the CMDs or CCD. For example, in the case of the $24 / 70 \mathrm{CMD}, 447$ objects are 
Table 7

Derived Properties of New Potential Taurus Members

\begin{tabular}{|c|c|c|c|c|c|c|c|c|c|c|c|c|}
\hline SST Tau Name & Common Name & $\begin{array}{c}\text { Our } \\
\text { Rank }\end{array}$ & $\begin{array}{c}\mathrm{c} 2 \mathrm{~d} \\
\text { Category }\end{array}$ & $\begin{array}{c}\text { Gutermuth } \\
\text { Category }\end{array}$ & $\begin{array}{c}\text { Our } \\
\text { Class }\end{array}$ & Spectral Type & $\begin{array}{c}\text { Our H } \alpha \\
\text { EQW }(\AA)\end{array}$ & $\begin{array}{c}A_{V} \\
(\mathrm{mag})\end{array}$ & $\begin{array}{c}\log L_{*} \\
\left(L_{\odot}\right)\end{array}$ & $L_{\mathrm{IR}} / L_{\text {total }}$ & $\begin{array}{l}\text { Adopted Memb. } \\
\text { Classification }\end{array}$ & Notes \\
\hline $041159.7+294236$ & $\ldots$ & $\mathrm{C}$ & 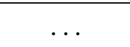 & $\ldots$ & III & G8-K1 & 0.9 & 2.8 & -0.8 & 0.0020 & Needs add'l follow up & $\mathrm{b}$ \\
\hline $041332.3+291726$ & $\ldots$ & A & Not YSO & Star & II & $\ldots$ & $\ldots$ & $\ldots$ & $\ldots$ & $\ldots$ & Pending follow up & $\mathrm{c}$ \\
\hline 041339.4+292114 & $\ldots$ & B- & YSO & Embedded & Flat & $\ldots$ & $\ldots$ & $\ldots$ & $\ldots$ & & Pending follow up & $\mathrm{j}$ \\
\hline $041427.3+255130$ & $\ldots$ & $\mathrm{B}+$ & Not YSO & Star & III & G5 & 2.5 & 0.4 & -1.7 & 0.0095 & Possible new member & $\mathrm{bn}$ \\
\hline $041535.6+284741$ & $\ldots$ & $\mathrm{A}-$ & YSO & Embedded & $\mathrm{I}$ & & $\ldots$ & $\ldots$ & 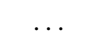 & & Pending follow up & $\mathrm{c}$ \\
\hline $041539.1+281858$ & $\ldots$ & $\mathrm{A}+$ & YSO & Class II & II & M5 & -7.2 & 2.8 & -0.5 & 0.075 & New member & $\mathrm{b} f \mathrm{l}$ \\
\hline $041542.7+290959$ & $\ldots$ & $\mathrm{A}+$ & YSO & Star & II & $\mathrm{M} 0 \mathrm{w} / \mathrm{Av}$ & -1.9 & 2.5 & -0.5 & 0.099 & New member & $\mathrm{bf}$ \\
\hline $041557.9+274617$ & $\ldots$ & $\mathrm{A}+$ & YSO & Class II & II & M6 & -32.2 & 1.1 & -1.1 & 0.12 & New member & $\mathrm{b} f \mathrm{l}$ \\
\hline $041604.8+261801$ & $\ldots$ & $\mathrm{C}-$ & YSO & Embedded & Flat & $\ldots$ & $\ldots$ & $\ldots$ & $\ldots$ & $\ldots$ & Pending follow up & $\ldots$ \\
\hline $041605.8+281426$ & $\ldots$ & $\mathrm{C}$ & Not YSO & Contam. & I & $\ldots$ & $\ldots$ & $\ldots$ & $\ldots$ & $\ldots$ & Pending follow up & $\ldots$ \\
\hline $041624.5+290858$ & HD 281820 & B & YSO & Star & III & A2 & 11.5 & 0.8 & 0.3 & 0.00017 & Needs add'l follow up & $\mathrm{bn}$ \\
\hline $041706.2+264413$ & $\ldots$ & $\mathrm{B}+$ & Not YSO & Star & III & K3 & 1.5 & 0.9 & -1.0 & 0.0020 & Needs add'1 follow up & $\mathrm{b}$ \\
\hline $041801.1+283526$ & $\ldots$ & $\mathrm{B}+$ & Not YSO & Star & III & K0-K2: & 1.1 & 6.2 & -0.9 & 0.0013 & Possible new member & bo \\
\hline $041803.3+244009$ & $\ldots$ & $\mathrm{B}$ & YSO & Star & III & A9 & 7.9 & 0.3 & -0.3 & 0.0022 & Needs add'l follow up & b e $n$ \\
\hline $041810.5+284447$ & $\ldots$ & $\mathrm{C}$ & YSO & Class II & I & $\ldots$ & $\ldots$ & $\ldots$ & $\ldots$ & $\ldots$ & Pending follow up & $\ldots$ \\
\hline $041810.7+251957$ & [GBM90] L1506 1 & $\mathrm{~A}+$ & YSO & Class II & II & K8-M0 & -4.3 & 4.3 & -0.3 & 0.11 & New member & a befo \\
\hline $041823.2+251928$ & 2MASX $04182321+2519281$ & $\mathrm{~B}+$ & YSO & Class II & Flat & $\mathrm{G}$ & 1.9 & 7.7 & -1.8 & 0.34 & Needs add'l follow up & be $1 \mathrm{n}$ \\
\hline $041831.2+282617$ & $\ldots$ & $\mathrm{B}+$ & YSO & Class II & I & $\ldots$ & $\ldots$ & $\ldots$ & $\ldots$ & $\ldots$ & Pending follow up & $\mathrm{c}$ \\
\hline $041858.0+235031$ & $\ldots$ & $\mathrm{B}$ & YSO & Embedded & Flat & $\ldots$ & $\ldots$ & $\ldots$ & $\ldots$ & $\ldots$ & Pending follow up & $\mathrm{c}$ \\
\hline $041859.0+255740$ & $\ldots$ & $\mathrm{B}-$ & YSO & Embedded & I & $\ldots$ & $\ldots$ & $\ldots$ & $\ldots$ & $\ldots$ & Pending follow up & $\ldots$ \\
\hline $041936.2+265256$ & $\ldots$ & B & Not YSO & Star & III & G3 & 2.7 & 0.8 & -1.3 & 0.0032 & Needs add'l follow up & $\mathrm{bn}$ \\
\hline $041940.4+270100$ & $\ldots$ & $\mathrm{B}-$ & YSO & Embedded & I & $\ldots$ & $\ldots$ & $\ldots$ & $\ldots$ & & Pending follow up & g 1 \\
\hline $041941.4+271607$ & HH390 star & A & YSO & Class II & I & $<\mathrm{M} 0$ & $\ldots$ & 5.5 & -1.7 & 0.87 & Probable new member & $\mathrm{c} 1$ \\
\hline $041946.5+271255$ & $\ldots$ & B & YSO & Embedded & I & $\ldots$ & $\ldots$ & $\ldots$ & $\ldots$ & $\ldots$ & Pending follow up & $\mathrm{cf}$ \\
\hline $042016.1+282132$ & $\ldots$ & $\mathrm{A}+$ & YSO & Class II & II & M6e & -133.1 & 0.2 & -2.1 & 0.13 & New member & $\mathrm{bcfk}$ \\
\hline $042021.4+281349$ & $\ldots$ & $\mathrm{A}+$ & YSO & Class II & Flat & M0e & -489.1 & 3.5 & -2.4 & 0.44 & New member & $\mathrm{abcf} 1$ \\
\hline $042025.8+281923$ & $\ldots$ & $\mathrm{A}+$ & YSO & Class II & Flat & M4e & -102.2 & 11.2 & -1.5 & 0.71 & New member & $\mathrm{bcfk} 1$ \\
\hline $042025.8+281641$ & $\ldots$ & B & YSO & Star & III & G2: & 3.1 & 1.0 & -0.2 & 0.00016 & Needs add'l follow up & $\mathrm{b}$ \\
\hline $042026.0+280408$ & $\ldots$ & $\mathrm{A}+$ & YSO & Class II & II & M2 & -5.3 & 0.0 & -0.7 & 0.26 & New member & $\mathrm{bcfkl}$ \\
\hline $042109.3+275036$ & $\ldots$ & $\mathrm{A}+$ & YSO & Class II & III & M3-4e & -17.6 & 0.0 & -1.0 & 0.020 & New member & $\mathrm{bcfk} 1$ \\
\hline $042110.9+255259$ & V412 Tau & $\mathrm{B}$ & $\ldots$ & $\ldots$ & III & A1 & 9.2 & 8.3 & 2.1 & $7.1 \mathrm{e}-05$ & Needs add' 1 follow up & b 1 \\
\hline $042135.6+253835$ & $\ldots$ & B & Not YSO & Star & III & F6-G0 & 3.1 & 1.2 & -1.2 & 0.0043 & Possible new member & $\mathrm{bn}$ \\
\hline $042146.3+242505$ & $\ldots$ & $\mathrm{B}+$ & YSO & Star & III & M3 & 0.0 & 2.9 & 0.1 & $<0.00023$ & Needs add'l follow up & $\mathrm{bk}$ \\
\hline $042151.3+265720$ & $\ldots$ & B & .. & Star & II & $\ldots$ & $\ldots$ & $\ldots$ & $\ldots$ & $\ldots$ & Pending follow up & c \\
\hline $042200.9+235430$ & $\ldots$ & $\mathrm{B}+$ & YSO & Star & III & M3 & 0.0 & 3.7 & 0.2 & $<4.6 \mathrm{e}-05$ & Needs add' 1 follow up & b k 1 \\
\hline $042212.9+254659$ & $\ldots$ & $\mathrm{B}-$ & YSO & Class II & Flat & $\ldots$ & $\ldots$ & $\ldots$ & $\ldots$ & $\ldots$ & Pending follow up & e 1 \\
\hline $042215.6+265706$ & 2MASS J04221568+2657060 & $\mathrm{A}+$ & YSO & Class II & I & K8-M0 & -7.8 & 4.5 & -1.5 & 0.65 & New member & $\mathrm{bc} f \mathrm{gh}$ i 1 \\
\hline $042220.9+264248$ & $\ldots$ & A & YSO & Embedded & I & $\ldots$ & $\ldots$ & $\ldots$ & $\ldots$ & $\ldots$ & Pending follow up & $\ldots$ \\
\hline $042247.8+264553$ & IRAS04196+2638 & $\mathrm{A}+$ & YSO & Class II & II & M1 & $\ldots$ & 6.9 & -0.4 & 0.24 & New member & $\mathrm{d}$ f 1 \\
\hline $042254.6+282354$ & NSV 1577 & A & YSO & Star & II & $\mathrm{A} 0$ & 10.0 & 1.5 & 1.4 & 0.00060 & Probable new member & $\mathrm{b} \mathrm{m}$ \\
\hline $042306.0+280119$ & J04230607+2801194 & A+ & YSO & Class II & II & M6 & $\ldots$ & 0.0 & -1.5 & 0.10 & New member & $\mathrm{f} 1$ \\
\hline $042318.2+264115$ & J04231822+2641156 & A+ & YSO & Class II & II & M3.5 & $\ldots$ & 8.7 & -0.6 & 0.081 & New member & $\mathrm{f}$ \\
\hline $042325.9+250354$ & $\ldots$ & $\mathrm{C}-$ & YSO & Star & II & $\ldots$ & $\ldots$ & $\ldots$ & $\ldots$ & $\ldots$ & Pending follow up & $\mathrm{c}$ \\
\hline $042335.3+250302$ & FU Tau & $\mathrm{A}+$ & YSO & Class II & II & M5e & -88.4 & 1.9 & -0.7 & 0.18 & New member & $\mathrm{b} \mathrm{f} \mathrm{k} 1$ \\
\hline $042339.0+251855$ & $\ldots$ & $\mathrm{C}$ & Not YSO & Contam. & I & $\ldots$ & $\ldots$ & $\ldots$ & $\ldots$ & $\ldots$ & Pending follow up & $\ldots$ \\
\hline
\end{tabular}


Table 7

(Continued)

\begin{tabular}{|c|c|c|c|c|c|c|c|c|c|c|c|c|}
\hline SST Tau Name & Common Name & $\begin{array}{l}\text { Our } \\
\text { Rank }\end{array}$ & $\begin{array}{c}\mathrm{c} 2 \mathrm{~d} \\
\text { Category }\end{array}$ & $\begin{array}{c}\text { Gutermuth } \\
\text { Category }\end{array}$ & $\begin{array}{c}\text { Our } \\
\text { Class }\end{array}$ & Spectral Type & $\begin{array}{c}\text { Our } \mathrm{H} \alpha \\
\text { EQW }(\AA)\end{array}$ & $\begin{array}{c}A_{V} \\
(\mathrm{mag})\end{array}$ & $\begin{array}{c}\log L_{*} \\
\left(L_{\odot}\right)\end{array}$ & $L_{\mathrm{IR}} / L_{\text {total }}$ & $\begin{array}{l}\text { Adopted Memb. } \\
\text { Classification }\end{array}$ & Notes \\
\hline $042350.1+264006$ & FS 116 & $\mathrm{C}$ & & Star & III & $\mathrm{G} 6-\mathrm{K} 2 \mathrm{w} / \mathrm{Av}$ & 1.6 & 8.2 & -0.6 & $<0.0011$ & Needs add'l follow up & bo \\
\hline $042356.0+242705$ & HD 283663 & $\mathrm{~B}$ & YSO & Star & III & $\mathrm{A} 1$ & 6.0 & 1.6 & 0.3 & 0.00012 & Needs add'l follow up & $\mathrm{bn}$ \\
\hline $042358.6+244742$ & $\ldots$ & $\mathrm{B}-$ & $\ldots$ & Star & III & $\mathrm{K} 2$ & 2.5 & 0.0 & -1.0 & 0.0052 & Needs add'l follow up & $\mathrm{b}$ \\
\hline $042420.9+263051$ & $\mathrm{~J} 04242090+2630511$ & $\mathrm{~A}+$ & YSO & Class II & II & M6.5 & & 0.3 & -1.9 & 0.12 & New member & $\mathrm{f} 1$ \\
\hline $042423.2+265008$ & $\ldots$ & $\mathrm{A}$ & YSO & Star & III & M3 & -4.7 & 1.3 & -0.7 & 0.0019 & Possible new member & $\mathrm{bk} 1$ \\
\hline $042515.5+282927$ & $\ldots$ & $\mathrm{A}+$ & YSO & Star & III & M6e & -23.5 & 0.0 & -1.1 & $<0.00019$ & New member & $\mathrm{bk} 1$ \\
\hline $042518.6+255535$ & & $\mathrm{~B}$ & & & III & M5 & 0.0 & 3.7 & 0.5 & $<0.00013$ & Needs add'l follow up & be k \\
\hline $042519.1+234716$ & HD 27923 & $\mathrm{~B}-$ & YSO & Star & III & B9 & 8.6 & 0.1 & 1.2 & $4.0 \mathrm{e}-05$ & Needs add'l follow up & $\mathrm{b} \mathrm{m} \mathrm{n}$ \\
\hline $042558.8+273701$ & HD 283637 & $\mathrm{~B}-$ & YSO & Star & III & B9 & 7.0 & 2.1 & 0.6 & $3.5 \mathrm{e}-05$ & Needs add'l follow up & $\mathrm{bn}$ \\
\hline $042653.3+255858$ & $\ldots$ & $\mathrm{C}$ & Not YSO & Contam. & I & $\ldots$ & $\ldots$ & $\ldots$ & $\ldots$ & $\ldots$ & Pending follow up & $\ldots$ \\
\hline $042721.0+240829$ & $\ldots$ & $\mathrm{C}-$ & $\ldots$ & Star & III & G6 & 2.8 & 0.2 & -1.8 & 0.0042 & Needs add'l follow up & bj $n$ \\
\hline $042728.1+262323$ & $\ldots$ & $\mathrm{C}+$ & Not YSO & Star & III & $\mathrm{K} 1 \mathrm{w} / \mathrm{Av}$ & 1.3 & 0.0 & -1.6 & 0.0070 & Needs add'l follow up & $\mathrm{bn}$ \\
\hline $042730.2+244123$ & 2MASX $04273023+2441232$ & B- & YSO & Star & I & $\ldots$ & $\ldots$ & $\ldots$ & $\ldots$ & $\ldots$ & Pending follow up & $\mathrm{c}$ \\
\hline $042810.4+243553$ & $\ldots$ & $\mathrm{C}$ & Not YSO & Embedded & Flat & $\ldots$ & $\ldots$ & $\ldots$ & $\ldots$ & $\ldots$ & Pending follow up & $\ldots$ \\
\hline $042857.4+243607$ & $\ldots$ & A & YSO & Star & III & $\ldots$ & $\ldots$ & $\ldots$ & $\ldots$ & $\ldots$ & Pending follow up & $\mathrm{c}$ \\
\hline $042902.9+243140$ & $\ldots$ & $\mathrm{C}-$ & YSO & Star & III & $\ldots$ & $\ldots$ & $\ldots$ & $\ldots$ & $\ldots$ & Pending follow up & c \\
\hline $042905.2+261535$ & 2MASX $04290517+2615358$ & $\mathrm{~B}-$ & $\ldots$ & Star & II & $\ldots$ & $\ldots$ & $\ldots$ & $\ldots$ & $\ldots$ & Pending follow up & $\ldots$ \\
\hline $042916.2+285627$ & HD 283629 & $\mathrm{~B}+$ & YSO & Star & III & G6 & 2.5 & 0.5 & -0.1 & 0.00015 & Needs add'l follow up & $\mathrm{b}$ \\
\hline $042920.8+274207$ & IRAS $04262+2735$ & A & YSO & Class II & II & M6 & 0.0 & 4.0 & 0.4 & 0.040 & Possible new member & bc de ko \\
\hline $042932.0+243059$ & $\ldots$ & A & YSO & Embedded & I & $\ldots$ & $\ldots$ & $\ldots$ & $\ldots$ & $\ldots$ & Pending follow up & $\mathrm{cf}$ \\
\hline $042936.0+243555$ & J04293606+2435556 & $\mathrm{A}+$ & YSO & Class II & II & M3 & $\ldots$ & 6.6 & -0.1 & 0.048 & New member & $\mathrm{fg} \mathrm{h} 1$ \\
\hline $042949.9+284253$ & $\ldots$ & $\mathrm{C}-$ & Not YSO & Class II & Flat & $\ldots$ & $\ldots$ & $\ldots$ & $\ldots$ & $\ldots$ & Pending follow up & $\ldots$ \\
\hline $043004.7+283306$ & $\ldots$ & $\mathrm{B}+$ & YSO & Star & III & $\mathrm{K} 2$ & 1.6 & 1.3 & -0.4 & 0.00036 & Needs add'l follow up & $\mathrm{b}$ \\
\hline $043024.1+281916$ & $\ldots$ & $\mathrm{B}$ & YSO & Star & III & M5 & 0.0 & 5.1 & 0.5 & $<9.6 \mathrm{e}-05$ & Needs add'l follow up & be k \\
\hline $043034.2+252427$ & $\ldots$ & $\mathrm{B}+$ & Not YSO & Star & II & G5-G6 w/Av & 2.7 & 2.3 & -1.9 & 0.0085 & Needs add'l follow up & $\mathrm{bn}$ \\
\hline $043042.8+274329$ & $\ldots$ & $\mathrm{B}+$ & YSO & Star & III & M6 & 0.0 & 3.6 & 0.1 & $<0.00032$ & Needs add'l follow up & b e k \\
\hline $043044.7+263308$ & $\ldots$ & $\mathrm{B}+$ & YSO & Star & III & $\mathrm{K} 3$ & 1.1 & 2.2 & 0.5 & 0.00089 & Needs add'l follow up & b 1 \\
\hline $043131.4+230025$ & $\ldots$ & $\mathrm{B}-$ & $\ldots$ & Star & III & $\mathrm{K} 2$ & 1.5 & 1.7 & -0.7 & 0.0027 & Needs add'l follow up & $\mathrm{b}$ \\
\hline $043133.1+292856$ & $\ldots$ & $\mathrm{B}-$ & YSO & Star & III & $\mathrm{B} 9 \mathrm{w} / \mathrm{Av}$ & 10.7 & 2.6 & 0.5 & $3.3 e-05$ & Needs add'l follow up & $\mathrm{bn}$ \\
\hline $043141.2+293922$ & $\ldots$ & $\mathrm{C}-$ & $\ldots$ & Star & III & $\mathrm{K} 2$ & 1.2 & 1.4 & -1.7 & 0.011 & Needs add'l follow up & $\mathrm{bjn}$ \\
\hline $043145.0+285908$ & $\ldots$ & B & Not YSO & Star & III & F0 & 1.1 & 1.3 & -0.6 & 0.0010 & Needs add'l follow up & $\mathrm{b} \mathrm{n}$ \\
\hline $043213.6+251746$ & GSC 01833-00754 & $\mathrm{C}+$ & YSO & Star & III & A9 & 6.4 & 1.3 & -0.0 & $8.2 \mathrm{e}-05$ & Needs add'l follow up & $\mathrm{b} \mathrm{n}$ \\
\hline $043214.6+223742$ & $\ldots$ & $\mathrm{C}$ & Not YSO & Embedded & Flat & $\ldots$ & $\ldots$ & $\ldots$ & $\ldots$ & $\ldots$ & Pending follow up & $\ldots$ \\
\hline $043224.1+225108$ & $\ldots$ & $\mathrm{A}+$ & YSO & Class II & II & M5e & -14.4 & 1.5 & -1.0 & 0.064 & New member & $\mathrm{bcfk}$ \\
\hline $043225.1+264732$ & $\ldots$ & $\mathrm{B}-$ & Not YSO & Star & III & $\mathrm{K} 1 \mathrm{w} / \mathrm{Av}$ & 1.3 & 3.1 & -0.9 & 0.0017 & Needs add'l follow up & $\mathrm{b}$ \\
\hline $043228.1+271122$ & $\ldots$ & $\mathrm{B}$ & YSO & Star & III & M6 & 0.0 & 7.0 & 0.5 & $<4.0 \mathrm{e}-05$ & Needs add'l follow up & be k \\
\hline $043244.2+230224$ & $\ldots$ & $\ldots$ & Not YSO & Embedded & I & & & $\ldots$ & $\ldots$ & $\ldots$ & xgal & $\mathrm{c}$ \\
\hline $043249.3+225308$ & .. & A+ & $\ldots$ & Class II & II & early $\mathrm{K}$ & -25.9 & 7.1 & -0.0 & 0.030 & New member & $\mathrm{abfjo}$ \\
\hline $043256.4+222342$ & HD 284481 & B- & YSO & Star & III & A8 & 6.5 & 1.0 & 0.3 & $6.4 \mathrm{e}-05$ & Needs add'l follow up & $\mathrm{bn}$ \\
\hline $043304.2+292149$ & HD 282276 & A & YSO & Star & II & $\mathrm{B} 8 \mathrm{~V}$ & 5.7 & $\ldots$ & $\ldots$ & $\ldots$ & Needs add'l follow up & $\mathrm{cm}$ \\
\hline $043312.6+291250$ & HD 282277 & $\mathrm{~B}$ & YSO & Star & III & A7V & 6.9 & $\ldots$ & $\ldots$ & $\ldots$ & Needs add'l follow up & $\ldots$ \\
\hline $043316.6+262724$ & $\ldots$ & $\mathrm{B}+$ & YSO & Class II & Flat & $\ldots$ & $\ldots$ & $\ldots$ & $\ldots$ & $\ldots$ & Pending follow up & $\ldots$ \\
\hline $043326.2+224529$ & 2MASS J04332621+2245293 & A & YSO & Star & II & M4 & -6.8 & 5.9 & -0.6 & 0.015 & Probable new member & $\mathrm{bcfgk}$ \\
\hline $043339.0+222720$ & $\ldots$ & A+ & YSO & Class II & II & M1e & -23.7 & 7.9 & -0.9 & 0.12 & New member & $\mathrm{bcfk}$ \\
\hline $043341.8+223836$ & $\ldots$ & $\mathrm{B}-$ & $\ldots$ & Star & III & $\mathrm{K} 2 \mathrm{w} / \mathrm{Av}$ & 0.7 & 4.9 & -0.9 & $<0.0021$ & Needs add'l follow up & $\mathrm{b}$ \\
\hline
\end{tabular}


Table 7

\begin{tabular}{|c|c|c|c|c|c|c|c|c|c|c|c|c|}
\hline SST Tau Name & Common Name & $\begin{array}{c}\text { Our } \\
\text { Rank }\end{array}$ & $\begin{array}{c}\mathrm{c} 2 \mathrm{~d} \\
\text { Category }\end{array}$ & $\begin{array}{l}\text { Gutermuth } \\
\text { Category }\end{array}$ & $\begin{array}{c}\text { Our } \\
\text { Class }\end{array}$ & Spectral Type & $\begin{array}{c}\text { Our } \mathrm{H} \alpha \\
\text { EQW }(\AA)\end{array}$ & $\begin{array}{c}A_{V} \\
(\mathrm{mag}) \\
\end{array}$ & $\begin{array}{c}\log L_{*} \\
\left(L_{\odot}\right)\end{array}$ & $L_{\mathrm{IR}} / L_{\text {total }}$ & $\begin{array}{l}\text { Adopted Memb. } \\
\text { Classification }\end{array}$ & Notes \\
\hline $043344.6+261500$ & & A+ & YSO & Class II & II & M6e & -82.2 & 5.4 & -0.6 & 0.13 & New member & b c e f k 1 \\
\hline $043349.5+291528$ & IRAS 04306+2909 & $\ldots$ & YSO & Embedded & I & xgal & -1.9 & 9.5 & 0.2 & 0.021 & xgal & $\mathrm{bd}$ \\
\hline $043352.4+261254$ & J04335245+2612548 & A+ & YSO & Class II & II & M8.5 & $\ldots$ & 4.9 & -2.4 & 0.13 & New member & $\mathrm{f}$ \\
\hline $043359.2+293636$ & $\ldots$ & $\mathrm{B}+$ & YSO & Star & III & M3 w/Av & 0.0 & 5.5 & 0.4 & $<8.0 \mathrm{e}-05$ & Needs add'l follow up & b k \\
\hline $043419.5+265210$ & $\ldots$ & A & Not YSO & Star & III & $\ldots$ & $\ldots$ & $\ldots$ & $\ldots$ & 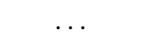 & Pending follow up & $\mathrm{c}$ \\
\hline $043419.8+232649$ & HD 284530 & $\mathrm{~B}-$ & YSO & Star & III & B8 & 7.7 & 1.9 & 1.2 & $3.8 \mathrm{e}-05$ & Needs add'l follow up & $\mathrm{b} \mathrm{m} \mathrm{n}$ \\
\hline $043435.4+264406$ & $\ldots$ & $\mathrm{B}-$ & $\ldots$ & $\ldots$ & III & M3 & 1.4 & 5.8 & 0.6 & 0.0010 & Needs add'l follow up & be k \\
\hline $043452.5+240244$ & $\ldots$ & $\mathrm{B}+$ & Not YSO & Star & III & $\mathrm{K} 2 \mathrm{w} / \mathrm{Av}$ & 1.6 & 4.4 & -1.1 & 0.0038 & Needs add'l follow up & $\mathrm{b}$ \\
\hline $043456.9+225835$ & 2MASS J04345693+2258358 & $A+$ & YSO & Star & III & M0 & -4.5 & 1.8 & -0.5 & 0.00023 & New member & $\mathrm{b} f \mathrm{~g} \mathrm{i}$ \\
\hline $043521.3+255510$ & & $\ldots$ & YSO & Embedded & Flat & ... & .. & $\ldots$ & 然 & & xgal & c \\
\hline $043542.0+225222$ & 2MASS J04354203+2252226 & $\mathrm{A}+$ & YSO & Star & III & M5e & -18.6 & 2.1 & -0.8 & $<0.00014$ & New member & $\mathrm{b} f \mathrm{gik}$ \\
\hline $043557.6+225357$ & J04355760+2253574 & $\mathrm{A}+$ & YSO & Star & II & M5:e & -30.4 & 3.4 & -2.3 & 0.15 & New member & $\mathrm{bck}$ \\
\hline $043559.4+223829$ & $\ldots$ & $\mathrm{A}+$ & YSO & Class II & Flat & K7-M0 & -1.7 & 2.0 & -2.0 & 0.53 & New member & $\mathrm{b}$ \\
\hline $043621.4+271912$ & $\ldots$ & A- & YSO & Star & II & $\mathrm{K} 2 \mathrm{e}$ & 0.8 & 2.6 & -1.5 & 0.012 & Possible new member & b no \\
\hline $043621.5+235116$ & J04362151+2351165 & $\mathrm{A}+$ & YSO & Class II & II & M5.25 & .. & 0.1 & -1.8 & 0.065 & New member & $\mathrm{cf}$ \\
\hline $043636.1+265910$ & $\ldots$ & B & & Star & III & K2 & 1.0 & 1.1 & -1.4 & 0.0078 & Needs add'l follow up & $\mathrm{b}$ \\
\hline $043642.0+265339$ & $\ldots$ & B & YSO & Embedded & I & $\ldots$ & $\ldots$ & $\ldots$ & $\ldots$ & & Pending follow up & $\ldots$ \\
\hline $043720.8+250019$ & $\ldots$ & $\mathrm{B}+$ & YSO & Star & III & G0 & 3.5 & 0.8 & -0.3 & 0.00035 & Needs add'l follow up & $\mathrm{b}$ \\
\hline $043724.8+270919$ & SV* SVS 1085 & B & YSO & Class II & III & B8e & -20.4 & 2.3 & 0.7 & 0.00056 & background $\mathrm{Be}$ & $\mathrm{bc}$ \\
\hline $043756.7+254622$ & ITG 1 & $\mathrm{~A}+$ & YSO & Class II & II & $\ldots$ & $\ldots$ & $\ldots$ & $\ldots$ & $\ldots$ & New member & $\mathrm{cdf}$ \\
\hline $043801.9+251926$ & $\ldots$ & $\mathrm{A}+$ & Not YSO & Star & II & K8 & -1.9 & 3.3 & -1.6 & 0.015 & New member & $\mathrm{bc}$ \\
\hline $043803.6+221223$ & $\ldots$ & $\mathrm{C}-$ & Not YSO & Star & III & G0 & 3.2 & 0.3 & -1.0 & 0.0020 & Needs add'l follow up & $\mathrm{bn}$ \\
\hline $043816.5+261450$ & $\ldots$ & $\mathrm{C}-$ & YSO & Star & III & $\ldots$ & .. & $\ldots$ & $\ldots$ & & Pending follow up & c \\
\hline $043826.7+265501$ & $\ldots$ & $\mathrm{B}+$ & YSO & Star & III & G6-K2 & 1.8 & 1.0 & -0.4 & 0.00019 & Needs add'l follow up & b \\
\hline $043905.2+233745$ & J04390525+2337450 & $\mathrm{A}+$ & YSO & Class II & Flat & $\mathrm{K} 5 \mathrm{e}$ & -28.2 & 4.1 & -1.3 & 0.39 & New member & $\mathrm{bcf}$ \\
\hline $043933.6+235921$ & J04393364+2359212 & $\mathrm{A}+$ & YSO & Class II & II & M5 & $\ldots$ & 1.3 & -1.0 & 0.15 & New member & $\mathrm{f}$ \\
\hline $043939.9+252034$ & JH 225 & B- & YSO & Star & III & $\mathrm{B} 9 \mathrm{w} / \mathrm{Av}$ & 10.5 & 4.1 & 0.9 & $5.0 \mathrm{e}-05$ & Needs add'l follow up & be $n$ \\
\hline $043943.8+271956$ & & $\mathrm{C}$ & $\ldots$ & Star & III & $\mathrm{K} 0-\mathrm{K} 2$ & 1.2 & 2.5 & -0.9 & $<0.0014$ & Needs add'l follow up & $\mathrm{b}$ \\
\hline $043944.8+260152$ & ITG 15 & $\mathrm{~A}+$ & YSO & Class II & II & M5 & $\cdots$ & 4.4 & -0.3 & 0.072 & New member & $\mathrm{d}$ f 1 \\
\hline $044000.6+235821$ & J04400067+2358211 & $\mathrm{A}+$ & YSO & Class II & II & M6 & $\cdots$ & 0.0 & -1.5 & 0.096 & New member & $\mathrm{f}$ \\
\hline $044022.8+243307$ & $\cdots$ & $\ldots$ & YSO & Embedded & I & $\ldots$ & $\ldots$ & $\ldots$ & $\ldots$ & $\cdots$ & xgal & $\mathrm{c}$ \\
\hline $044023.0+255702$ & $\ldots$ & B- & YSO & Star & III & G0 & 1.0 & 7.4 & -0.5 & 0.00022 & Needs add'l follow up & 1 \\
\hline $044048.4+233941$ & $\ldots$ & A- & $\ldots$ & $\ldots$ & II & K0 & 0.8 & 2.1 & -1.9 & 0.012 & Possible new member & $\mathrm{bn}$ \\
\hline $044124.6+254353$ & ITG 40 & $\mathrm{~A}+$ & YSO & Class II & Flat & M3.5 & $\ldots$ & 22.2 & -0.8 & 0.060 & New member & $d f$ \\
\hline $044125.7+254349$ & $\ldots$ & B & Not YSO & Embedded & I & $\ldots$ & $\ldots$ & $\ldots$ & $\ldots$ & $\ldots$ & Pending follow up & e g \\
\hline $044200.4+235813$ & $\ldots$ & $\ldots$ & YSO & Embedded & I & $\ldots$ & $\ldots$ & $\cdots$ & $\ldots$ & $\cdots$ & xgal & $\mathrm{c}$ \\
\hline $044241.1+244117$ & CCDM J04427+2441AB & $\mathrm{B}+$ & YSO & Star & III & A0 & 9.8 & $\ldots$ & $\ldots$ & $\ldots$ & Needs add'l follow up & 1 \\
\hline $044253.9+253709$ & $\ldots$ & $\mathrm{B}$ & Not YSO & Star & III & F5 & 3.3 & 2.2 & -1.5 & 0.0043 & Needs add'l follow up & $\mathrm{n}$ \\
\hline $044315.8+235358$ & $\ldots$ & $\mathrm{C}-$ & YSO & Embedded & I & $\ldots$ & .. & $\ldots$ & $\ldots$ & & Pending follow up & $\ldots$ \\
\hline $044325.1+255706$ & $\ldots$ & $\mathrm{B}+$ & YSO & Star & III & K7-M0 & 0.5 & 0.2 & -0.5 & 0.00020 & Needs add'l follow up & b 1 \\
\hline $044345.3+243908$ & TYC 1834-591-1 & B & YSO & Star & III & $\ldots$ & $\ldots$ & $\ldots$ & $\ldots$ & $\ldots$ & Pending follow up & $\mathrm{cm}$ \\
\hline $044358.3+235103$ & $\ldots$ & $\mathrm{C}-$ & YSO & Star & III & M3 & 1.4 & 3.7 & 0.8 & $<9.5 \mathrm{e}-06$ & Needs add'l follow up & $\mathrm{b} \mathrm{k}$ \\
\hline $044518.2+242436$ & HD 30067 & $\mathrm{~B}+$ & YSO & Star & III & $\mathrm{A} 4 \mathrm{~V}$ & 10.6 & $\ldots$ & $\ldots$ & $\ldots$ & Needs add'l follow up & $1 \mathrm{~m}$ \\
\hline $044539.8+251704$ & $\ldots$ & $\mathrm{B}$ & YSO & Star & III & M5 & 0.0 & 4.9 & -0.1 & $<7.3 \mathrm{e}-05$ & Needs add'l follow up & b c e k 1 \\
\hline $044550.7+254448$ & $\ldots$ & $\ldots$ & YSO & Embedded & Flat & $\ldots$ & $\ldots$ & $\ldots$ & $\ldots$ & $\ldots$ & xgal & $\mathrm{c}$ \\
\hline
\end{tabular}


Table 7

(Continued)

\begin{tabular}{|c|c|c|c|c|c|c|c|c|c|c|c|c|}
\hline SST Tau Name & Common Name & $\begin{array}{l}\text { Our } \\
\text { Rank }\end{array}$ & $\begin{array}{c}\mathrm{c} 2 \mathrm{~d} \\
\text { Category }\end{array}$ & $\begin{array}{c}\text { Gutermuth } \\
\text { Category }\end{array}$ & $\begin{array}{c}\text { Our } \\
\text { Class } \\
\end{array}$ & Spectral Type & $\begin{array}{c}\text { Our H } \alpha \\
\text { EQW }(\AA)\end{array}$ & $\begin{array}{c}A_{V} \\
(\mathrm{mag})\end{array}$ & $\begin{array}{c}\log L_{*} \\
\left(L_{\odot}\right)\end{array}$ & $L_{\mathrm{IR}} / L_{\text {total }}$ & $\begin{array}{l}\text { Adopted Memb. } \\
\text { Classification }\end{array}$ & $\overline{\text { Notes }}$ \\
\hline $044555.7+261858$ & $\ldots$ & A & Not YSO & Star & II & K4 & 1.3 & 2.4 & -1.0 & 0.021 & Possible new member & $\mathrm{bc}$ \\
\hline $044557.0+244042$ & $\ldots$ & $\mathrm{B}-$ & $\ldots$ & Class II & III & K2 & 1.5 & 1.8 & -1.9 & 0.015 & Needs add'l follow up & b e $n$ \\
\hline $044609.6+245237$ & $\ldots$ & $\mathrm{A}-$ & Not YSO & Star & II & early G & 2.4 & 5.4 & -1.4 & 0.0043 & Possible new member & $\mathrm{bcn}$ \\
\hline $044639.8+242526$ & $\ldots$ & $\mathrm{C}-$ & $\ldots$ & $\ldots$ & III & M5 & 0.0 & 4.5 & 1.0 & $<0.00018$ & Needs add'l follow up & b e k 1 \\
\hline $044644.4+262306$ & $\ldots$ & $\mathrm{B}+$ & $\ldots$ & $\ldots$ & III & M4 & 0.0 & 3.7 & 0.8 & $<0.00018$ & Needs add'l follow up & b k 1 \\
\hline $044650.3+243815$ & $\ldots$ & $\mathrm{B}-$ & Not YSO & Star & III & $\mathrm{K} 0-\mathrm{K} 1 \mathrm{w} / \mathrm{Av}$ & 2.1 & 1.8 & -2.0 & 0.0079 & Needs add'l follow up & $\mathrm{bn}$ \\
\hline $044802.3+253359$ & $\ldots$ & B & YSO & Star & III & $\mathrm{K} 0-\mathrm{K} 1 \mathrm{w} / \mathrm{Av}$ & 0.0 & 5.9 & -0.4 & $8.0 \mathrm{e}-05$ & Needs add'l follow up & b 1 \\
\hline $044832.3+234746$ & $\ldots$ & $\mathrm{C}$ & YSO & Embedded & I & $\ldots$ & $\ldots$ & $\ldots$ & $\ldots$ & $\ldots$ & Pending follow up & \\
\hline $044857.4+255853$ & 2MASX $04485745+2558527$ & $\mathrm{~B}+$ & Not YSO & Class II & II & $\mathrm{K}$ & 0.0 & 5.0 & -1.9 & 0.015 & Possible new member & $\mathrm{bcn}$ \\
\hline $044900.1+241346$ & $\ldots$ & $\ldots$ & YSO & Contam. & I & $\ldots$ & $\ldots$ & $\ldots$ & $\ldots$ & $\ldots$ & xgal & $\mathrm{c}$ \\
\hline $044913.7+252549$ & $\ldots$ & $\mathrm{C}-$ & $\ldots$ & Contam. & III & F6 & 2.6 & 4.5 & -1.6 & 0.011 & Needs add'l follow up & $\mathrm{bn}$ \\
\hline $044916.3+243827$ & CCDM J04493+2438A & $\mathrm{C}$ & YSO & Star & III & $\mathrm{K} 2 \mathrm{w} / \mathrm{Av}$ & 1.6 & 0.5 & -0.2 & 0.00018 & Needs add'l follow up & $\mathrm{bj}$ \\
\hline $044941.5+254010$ & $\ldots$ & $\mathrm{B}+$ & Not YSO & Star & III & M3 & 0.0 & 1.5 & -2.0 & 0.011 & Needs add'l follow up & b c k \\
\hline
\end{tabular}

Notes.

${ }^{a}$ Ca IR triplet in emission in our spectrum.

${ }^{\mathrm{b}}$ New spectral type, reported here.

${ }^{c}$ Unreduced IR spectrum in-hand, to be discussed in a later paper

${ }^{\mathrm{d}}$ Object previously identified in the literature as a potential Taurus member.

${ }^{\mathrm{e}}$ Object previously identified in the literature as a potential non-member.

${ }^{\mathrm{f}}$ Object identified as a Taurus member by other authors using portions of this Spitzer Legacy data set.

${ }^{g}$ X-ray source in the XEST survey.

${ }^{\text {h }}$ Ultraviolet source in the XEST survey (OM data).

i Object found via X-rays in Scelsi et al. (2007) and confirmed as new member in Scelsi et al. (2008); object also independently rediscovered via IR excesses here.

${ }^{\mathrm{j}}$ High spatial-resolution optical images suggest IR flux density may be subject to confusion with a nearby object.

${ }^{\mathrm{k}}$ Gravity analysis (see the text) suggests intermediate gravity object and therefore likely member.

UV excess source in SDSS data.

${ }^{\mathrm{m}}$ Extended emission seen in image at $8 \mu \mathrm{m}$ or longer, which may be affecting the long-wavelength photometry.

${ }^{\mathrm{n}}$ Object appears lower in the HR diagram than most Taurus members; object may suffer from additional (unmodeled) extinction such as may be found in edge-on disks, but may also be background giant.

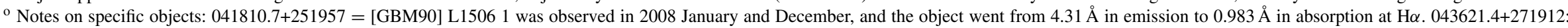

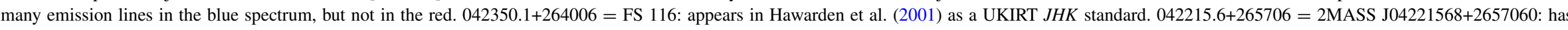

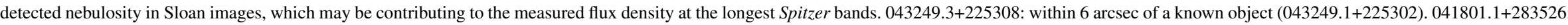
may be double source in CFHT image. $042920.8+274207$ : shares many characteristics of young stars, but also those of some post-main-sequence objects.

(This table is also available in a machine-readable form in the online journal.) 
Table 8

List of New Confirmed, Probable, Possible, and Pending Taurus Members

\begin{tabular}{|c|c|c|c|c|c|}
\hline SST Tau Name & Common Name & R.A. (J2000) & Decl. (J2000) & Our Rank & Distance $^{\mathrm{a}}(\operatorname{arcmin})$ \\
\hline \multicolumn{6}{|c|}{ New members } \\
\hline $041539.1+281858$ & $\ldots$ & 63.913178 & 28.316282 & $A+$ & 12.66 \\
\hline $041542.7+290959$ & $\ldots$ & 63.928283 & 29.166586 & $\mathrm{~A}+$ & 4.14 \\
\hline $041557.9+274617$ & $\ldots$ & 63.991642 & 27.771547 & $\mathrm{~A}+$ & 7.54 \\
\hline $041810.7+251957$ & [GBM90] L1506 1 & 64.544954 & 25.332630 & $\mathrm{~A}+$ & 62.64 \\
\hline $042016.1+282132$ & $\ldots$ & 65.067129 & 28.359041 & $\mathrm{~A}+$ & 10.67 \\
\hline $042021.4+281349$ & $\ldots$ & 65.089345 & 28.230326 & $\mathrm{~A}+$ & 16.91 \\
\hline $042025.8+281923$ & $\ldots$ & 65.107642 & 28.323265 & $\mathrm{~A}+$ & 13.65 \\
\hline $042026.0+280408$ & $\ldots$ & 65.108609 & 28.069153 & $\mathrm{~A}+$ & 17.42 \\
\hline $042109.3+275036$ & $\ldots$ & 65.288930 & 27.843567 & $\mathrm{~A}+$ & 11.17 \\
\hline $042215.6+265706$ & 2MASS J04221568+2657060 & 65.565341 & 26.951694 & $\mathrm{~A}+$ & 2.16 \\
\hline $042247.8+264553$ & IRAS $04196+2638$ & 65.699455 & 26.764736 & $\mathrm{~A}+$ & 5.35 \\
\hline $042306.0+280119$ & J04230607+2801194 & 65.775303 & 28.022081 & $\mathrm{~A}+$ & 4.65 \\
\hline $042318.2+264115$ & J04231822+2641156 & 65.825957 & 26.687672 & $\mathrm{~A}+$ & 13.16 \\
\hline $042335.3+250302$ & FU Tau & 65.897483 & 25.050739 & $A+$ & 6.86 \\
\hline $042420.9+263051$ & J04242090+2630511 & 66.087089 & 26.514210 & $\mathrm{~A}+$ & 13.83 \\
\hline $042515.5+282927$ & & 66.314616 & 28.490980 & $\mathrm{~A}+$ & 36.65 \\
\hline $042936.0+243555$ & J04293606+2435556 & 67.400276 & 24.598793 & $\mathrm{~A}+$ & 4.05 \\
\hline $043224.1+225108$ & $\ldots$ & 68.100646 & 22.852333 & $\mathrm{~A}+$ & 6.06 \\
\hline $043249.3+225308$ & $\ldots$ & 68.205751 & 22.885616 & $A+$ & 0.11 \\
\hline $043339.0+222720$ & $\ldots$ & 68.412730 & 22.455774 & $\mathrm{~A}+$ & 19.77 \\
\hline $043344.6+261500$ & $\ldots$ & 68.436051 & 26.250147 & $\mathrm{~A}+$ & 2.74 \\
\hline $043352.4+261254$ & $\mathrm{~J} 04335245+2612548$ & 68.468574 & 26.215237 & $\mathrm{~A}+$ & 0.74 \\
\hline $043456.9+225835$ & 2MASS J04345693+2258358 & 68.737218 & 22.976627 & $\mathrm{~A}+$ & 6.93 \\
\hline $043542.0+225222$ & 2MASS J04354203+2252226 & 68.925159 & 22.872963 & $A+$ & 2.11 \\
\hline $043557.6+225357$ & J04355760+2253574 & 68.990035 & 22.899303 & $\mathrm{~A}+$ & 0.67 \\
\hline $043559.4+223829$ & $\ldots$ & 68.997913 & 22.641430 & $\mathrm{~A}+$ & 0.17 \\
\hline $043621.5+235116$ & J04362151+2351165 & 69.089655 & 23.854609 & $\mathrm{~A}+$ & 20.04 \\
\hline $043756.7+254622$ & ITG 1 & 69.486267 & 25.773035 & $\mathrm{~A}+$ & 12.61 \\
\hline $043801.9+251926$ & $\ldots$ & 69.507964 & 25.324060 & $\mathrm{~A}+$ & 28.65 \\
\hline $043905.2+233745$ & J04390525+2337450 & 69.771877 & 23.629189 & $\mathrm{~A}+$ & 1.89 \\
\hline $043933.6+235921$ & J04393364+2359212 & 69.890187 & 23.989231 & $\mathrm{~A}+$ & 24.14 \\
\hline $043944.8+260152$ & ITG 15 & 69.937014 & 26.031330 & $\mathrm{~A}+$ & 0.62 \\
\hline $044000.6+235821$ & J04400067+2358211 & 70.002817 & 23.972546 & $\mathrm{~A}+$ & 25.99 \\
\hline $044124.6+254353$ & ITG 40 & 70.352685 & 25.731396 & $\mathrm{~A}+$ & 3.82 \\
\hline \multicolumn{6}{|c|}{ Probable new members } \\
\hline $041941.4+271607$ & HH390 star & 64.922838 & 27.268616 & A & 2.52 \\
\hline $042254.6+282354$ & NSV 1577 & 65.727597 & 28.398354 & $\mathrm{~A}$ & 11.46 \\
\hline $043326.2+224529$ & 2MASS J04332621+2245293 & 68.359223 & 22.758150 & $\mathrm{~A}$ & 1.97 \\
\hline \multicolumn{6}{|c|}{ Possible new members } \\
\hline $042423.2+265008$ & & 66.096734 & 26.835686 & $\mathrm{~A}$ & 0.78 \\
\hline $042920.8+274207$ & IRAS $04262+2735$ & 67.336817 & 27.702072 & A & 13.68 \\
\hline $044555.7+261858$ & $\ldots$ & 71.482339 & 26.316357 & A & 61.92 \\
\hline $043621.4+271912$ & $\ldots$ & 69.089458 & 27.320208 & $\mathrm{~A}-$ & 19.72 \\
\hline $044048.4+233941$ & $\ldots$ & 70.202020 & 23.661591 & $\mathrm{~A}-$ & 24.00 \\
\hline $044609.6+245237$ & $\ldots$ & 71.540265 & 24.877144 & $\mathrm{~A}-$ & 9.85 \\
\hline $041427.3+255130$ & $\ldots$ & 63.613782 & 25.858398 & $\mathrm{~B}+$ & 55.12 \\
\hline $041801.1+283526$ & $\ldots$ & 64.504653 & 28.590672 & $\mathrm{~B}+$ & 5.44 \\
\hline $044857.4+255853$ & 2MASX $04485745+2558527$ & 72.239482 & 25.981394 & $\mathrm{~B}+$ & 67.12 \\
\hline $042135.6+253835$ & $\ldots$ & 65.398398 & 25.643232 & $\mathrm{~B}$ & 14.03 \\
\hline \multicolumn{6}{|c|}{ Needs additional follow up (beyond the spectra we have) } \\
\hline $043304.2+292149$ & HD 282276 & 68.267613 & 29.363867 & A & 101.91 \\
\hline $041706.2+264413$ & & 64.276248 & 26.737167 & $\mathrm{~B}+$ & 31.11 \\
\hline $041823.2+251928$ & 2MASX $04182321+2519281$ & 64.596722 & 25.324471 & $\mathrm{~B}+$ & 60.42 \\
\hline $042146.3+242505$ & $\ldots$ & 65.442927 & 24.418116 & $\mathrm{~B}+$ & 40.35 \\
\hline $042200.9+235430$ & $\ldots$ & 65.503948 & 23.908342 & $\mathrm{~B}+$ & 65.66 \\
\hline $042916.2+285627$ & HD 283629 & 67.317694 & 28.940914 & $\mathrm{~B}+$ & 61.49 \\
\hline $043004.7+283306$ & $\ldots$ & 67.519643 & 28.551838 & $\mathrm{~B}+$ & 40.58 \\
\hline $043034.2+252427$ & $\ldots$ & 67.642795 & 25.407726 & $\mathrm{~B}+$ & 20.43 \\
\hline $043042.8+274329$ & $\ldots$ & 67.678529 & 27.724981 & $\mathrm{~B}+$ & 25.37 \\
\hline $043044.7+263308$ & $\ldots$ & 67.686434 & 26.552361 & $\mathrm{~B}+$ & 13.42 \\
\hline $043359.2+293636$ & $\ldots$ & 68.497083 & 29.610262 & $\mathrm{~B}+$ & 120.82 \\
\hline $043452.5+240244$ & $\ldots$ & 68.718796 & 24.045792 & $\mathrm{~B}+$ & 11.26 \\
\hline $043720.8+250019$ & $\ldots$ & 69.336752 & 25.005396 & $\mathrm{~B}+$ & 36.60 \\
\hline $043826.7+265501$ & $\ldots$ & 69.611576 & 26.917101 & $\mathrm{~B}+$ & 43.44 \\
\hline
\end{tabular}


Table 8

(Continued.)

\begin{tabular}{|c|c|c|c|c|c|}
\hline SST Tau Name & Common Name & R.A. (J2000) & Decl. (J2000) & Our Rank & Distance $^{\mathrm{a}}(\operatorname{arcmin})$ \\
\hline $044241.1+244117$ & CCDM J04427+2441AB & 70.671643 & 24.688299 & $\mathrm{~B}+$ & 24.00 \\
\hline $044325.1+255706$ & $\ldots$ & 70.854940 & 25.951765 & $\mathrm{~B}+$ & 23.92 \\
\hline $044518.2+242436$ & HD 30067 & 71.325984 & 24.410131 & $\mathrm{~B}+$ & 39.42 \\
\hline $044644.4+262306$ & $\ldots$ & 71.685220 & 26.385118 & $\mathrm{~B}+$ & 73.58 \\
\hline $044941.5+254010$ & $\ldots$ & 72.423263 & 25.669575 & $\mathrm{~B}+$ & 57.67 \\
\hline $041624.5+290858$ & HD 281820 & 64.102356 & 29.149654 & $\mathrm{~B}$ & 10.65 \\
\hline $041803.3+244009$ & $\ldots$ & 64.514100 & 24.669361 & B & 77.88 \\
\hline $041936.2+265256$ & $\ldots$ & 64.900850 & 26.882286 & B & 13.40 \\
\hline $042025.8+281641$ & $\ldots$ & 65.107869 & 28.278227 & B & 15.39 \\
\hline $042110.9+255259$ & V412 Tau & 65.295779 & 25.883303 & B & 15.21 \\
\hline $042356.0+242705$ & HD 283663 & 65.983654 & 24.451431 & B & 29.40 \\
\hline $042518.6+255535$ & $\ldots$ & 66.327763 & 25.926657 & B & 16.52 \\
\hline $043024.1+281916$ & $\ldots$ & 67.600602 & 28.321260 & B & 30.42 \\
\hline $043145.0+285908$ & $\ldots$ & 67.937661 & 28.985607 & B & 73.56 \\
\hline $043228.1+271122$ & $\ldots$ & 68.117315 & 27.189692 & B & 15.88 \\
\hline $043312.6+291250$ & HD 282277 & 68.302720 & 29.214066 & B & 95.45 \\
\hline $043636.1+265910$ & $\ldots$ & 69.150616 & 26.986322 & B & 39.68 \\
\hline $044253.9+253709$ & $\ldots$ & 70.724809 & 25.619368 & B & 15.05 \\
\hline $044539.8+251704$ & $\ldots$ & 71.416097 & 25.284595 & B & 17.13 \\
\hline $044802.3+253359$ & $\ldots$ & 72.009726 & 25.566454 & B & 39.31 \\
\hline $042358.6+244742$ & $\ldots$ & 65.994199 & 24.795076 & $\mathrm{~B}-$ & 9.60 \\
\hline $042519.1+234716$ & HD 27923 & 66.329856 & 23.788006 & $\mathrm{~B}-$ & 34.93 \\
\hline $042558.8+273701$ & HD 283637 & 66.495259 & 27.617020 & $\mathrm{~B}-$ & 28.58 \\
\hline $043131.4+230025$ & $\ldots$ & 67.880924 & 23.007208 & $\mathrm{~B}-$ & 9.47 \\
\hline $043133.1+292856$ & $\ldots$ & 67.888044 & 29.482388 & $\mathrm{~B}-$ & 99.66 \\
\hline $043225.1+264732$ & $\ldots$ & 68.104744 & 26.792301 & $\mathrm{~B}-$ & 20.46 \\
\hline $043256.4+222342$ & HD 284481 & 68.235238 & 22.395138 & $\mathrm{~B}-$ & 23.30 \\
\hline $043341.8+223836$ & $\ldots$ & 68.424276 & 22.643473 & $\mathrm{~B}-$ & 9.54 \\
\hline $043419.8+232649$ & HD 284530 & 68.582806 & 23.447161 & $\mathrm{~B}-$ & 18.83 \\
\hline $043435.4+264406$ & $\ldots$ & 68.647885 & 26.735069 & $\mathrm{~B}-$ & 31.98 \\
\hline $043939.9+252034$ & JH 225 & 69.916487 & 25.342842 & $\mathrm{~B}-$ & 13.60 \\
\hline $044023.0+255702$ & $\ldots$ & 70.095875 & 25.950682 & $\mathrm{~B}-$ & 4.81 \\
\hline $044557.0+244042$ & $\ldots$ & 71.487694 & 24.678431 & $\mathrm{~B}-$ & 21.06 \\
\hline $044650.3+243815$ & $\ldots$ & 71.709980 & 24.637739 & $\mathrm{~B}-$ & 20.87 \\
\hline $042728.1+262323$ & $\ldots$ & 66.867199 & 26.389803 & $\mathrm{C}+$ & 7.71 \\
\hline $043213.6+251746$ & GSC 01833-00754 & 68.056868 & 25.296129 & $\mathrm{C}+$ & 10.62 \\
\hline $041159.7+294236$ & $\ldots$ & 62.998870 & 29.710224 & $\mathrm{C}$ & 35.29 \\
\hline $042350.1+264006$ & FS 116 & 65.958819 & 26.668579 & $\mathrm{C}$ & 12.67 \\
\hline $043943.8+271956$ & $\ldots$ & 69.932822 & 27.332352 & $\mathrm{C}$ & 55.67 \\
\hline $044916.3+243827$ & CCDM J04493+2438A & 72.318089 & 24.640902 & $\mathrm{C}$ & 40.52 \\
\hline $042721.0+240829$ & $\ldots$ & 66.837538 & 24.141438 & $\mathrm{C}-$ & 12.40 \\
\hline $043141.2+293922$ & $\ldots$ & 67.921919 & 29.656178 & $\mathrm{C}-$ & 110.09 \\
\hline $043803.6+221223$ & $\ldots$ & 69.515227 & 22.206503 & $\mathrm{C}-$ & 19.22 \\
\hline $044358.3+235103$ & $\cdots$ & 70.992959 & 23.850939 & $\mathrm{C}-$ & 68.89 \\
\hline $044639.8+242526$ & $\cdots$ & 71.666086 & 24.423908 & $\mathrm{C}-$ & 33.63 \\
\hline $044913.7+252549$ & $\ldots$ & 72.307325 & 25.430412 & $\mathrm{C}-$ & 43.42 \\
\hline \multicolumn{6}{|c|}{ Pending (spectroscopic) follow up } \\
\hline $041332.3+291726$ & $\ldots$ & 63.384823 & 29.290760 & $\mathrm{~A}$ & 5.52 \\
\hline $042220.9+264248$ & $\ldots$ & 65.587113 & 26.713463 & A & 3.69 \\
\hline $042857.4+243607$ & $\ldots$ & 67.239295 & 24.602066 & A & 6.74 \\
\hline $042932.0+243059$ & $\cdots$ & 67.383731 & 24.516598 & A & 2.77 \\
\hline $043419.5+265210$ & $\ldots$ & 68.581613 & 26.869499 & A & 39.11 \\
\hline $041535.6+284741$ & $\ldots$ & 63.898611 & 28.794920 & $\mathrm{~A}-$ & 17.79 \\
\hline $041831.2+282617$ & $\ldots$ & 64.630173 & 28.438248 & $\mathrm{~B}+$ & 0.47 \\
\hline $043316.6+262724$ & $\ldots$ & 68.319252 & 26.456673 & $\mathrm{~B}+$ & 11.46 \\
\hline $041858.0+235031$ & $\ldots$ & 64.741993 & 23.841991 & $\mathrm{~B}$ & 91.73 \\
\hline $041946.5+271255$ & $\ldots$ & 64.944061 & 27.215336 & B & 1.13 \\
\hline $042151.3+265720$ & $\ldots$ & 65.463854 & 26.955629 & B & 2.10 \\
\hline $043642.0+265339$ & $\ldots$ & 69.175240 & 26.894352 & B & 45.35 \\
\hline $044125.7+254349$ & $\ldots$ & 70.357346 & 25.730341 & B & 4.04 \\
\hline $044345.3+243908$ & TYC 1834-591-1 & 70.938928 & 24.652491 & $\mathrm{~B}$ & 34.45 \\
\hline $041339.4+292114$ & $\ldots$ & 63.414342 & 29.353973 & $\mathrm{~B}-$ & 4.88 \\
\hline $041401.7+280857$ & $\cdots$ & 63.507256 & 28.149384 & $\mathrm{~B}-$ & 2.35 \\
\hline $041859.0+255740$ & $\cdots$ & 64.745898 & 25.961126 & $\mathrm{~B}-$ & 45.20 \\
\hline $041940.4+270100$ & $\ldots$ & 64.918731 & 27.016882 & $\mathrm{~B}-$ & 9.79 \\
\hline $042212.9+254659$ & $\ldots$ & 65.553995 & 25.783281 & $\mathrm{~B}-$ & 2.34 \\
\hline
\end{tabular}


Table 8

(Continued.)

\begin{tabular}{|c|c|c|c|c|c|}
\hline SST Tau Name & Common Name & R.A. (J2000) & Decl. (J2000) & Our Rank & Distance $^{\mathrm{a}}(\operatorname{arcmin})$ \\
\hline $042730.2+244123$ & 2MASX $04273023+2441232$ & 66.876130 & 24.689869 & $\mathrm{~B}-$ & 8.02 \\
\hline $042905.2+261535$ & 2MASX 04290517+2615358 & 67.271721 & 26.259964 & $\mathrm{~B}-$ & 5.64 \\
\hline $043523.7+240450$ & $\ldots$ & 68.848785 & 24.080612 & $\mathrm{~B}-$ & 4.39 \\
\hline $041331.6+280613$ & $\ldots$ & 63.381946 & 28.103617 & $\mathrm{C}$ & 7.03 \\
\hline $041605.8+281426$ & $\ldots$ & 64.024513 & 28.240591 & $\mathrm{C}$ & 8.41 \\
\hline $041810.5+284447$ & $\ldots$ & 64.544147 & 28.746483 & $\mathrm{C}$ & 13.68 \\
\hline $042339.0+251855$ & $\ldots$ & 65.912544 & 25.315405 & $\mathrm{C}$ & 22.69 \\
\hline $042653.3+255858$ & $\ldots$ & 66.722092 & 25.982811 & $\mathrm{C}$ & 6.87 \\
\hline $042810.4+243553$ & $\ldots$ & 67.043381 & 24.598303 & $\mathrm{C}$ & 16.92 \\
\hline $043214.6+223742$ & $\ldots$ & 68.060855 & 22.628393 & $\mathrm{C}$ & 15.59 \\
\hline $044832.3+234746$ & $\ldots$ & 72.134884 & 23.796349 & $\mathrm{C}$ & 75.53 \\
\hline $041604.8+261801$ & $\ldots$ & 64.020153 & 26.300299 & $\mathrm{C}-$ & 33.29 \\
\hline $042325.9+250354$ & $\ldots$ & 65.858228 & 25.065123 & $\mathrm{C}-$ & 8.24 \\
\hline $042902.9+243140$ & $\ldots$ & 67.262433 & 24.527840 & $\mathrm{C}-$ & 4.90 \\
\hline $042949.9+284253$ & $\ldots$ & 67.458318 & 28.714808 & $\mathrm{C}-$ & 49.05 \\
\hline $043816.5+261450$ & $\cdots$ & 69.569150 & 26.247238 & $\mathrm{C}-$ & 3.19 \\
\hline $044315.8+235358$ & $\ldots$ & 70.816129 & 23.899599 & $\mathrm{C}-$ & 60.39 \\
\hline
\end{tabular}

Note. ${ }^{a}$ Angular distance to nearest previously identified Taurus member.

selected by our color cuts in this diagram. Of these, $19 \%$ are previously identified YSOs, $7 \%$ are new candidate YSOs, $10 \%$ are previously identified non-members, and $60 \%$ are new nonmembers. And, out of the 215 stars that compose our previously identified Taurus member sample, $41 \%$ are recovered in the $24 / 70$ diagram, of the 148 objects in our candidate YSO sample, $22 \%$ are found here, of the 821 previously identified nonmembers, $6 \%$ are found in this diagram, and of the 489 new nonmembers, $55 \%$ are found here. This table reveals that the $K_{\mathrm{s}} / 24$ diagram recovers the highest fraction of previously identified objects and of new candidate objects. The 24/70 CMD finds the highest fraction of new non-members (by fraction of objects found in this diagram as well as by fraction of the entire new nonmember list); this is not particularly surprising, as the objects that are detected at $70 \mu \mathrm{m}$ tend to be either YSOs or extragalactic objects. This table also shows that the $\sim 6^{\circ}$ ELAIS N1 SWIRE sample is largely found outside of our color selection criteria; few galaxies of the sort found in this SWIRE sample are likely to be selected by our criteria.

Table 3 shows, of the entire sample of previously identified Taurus members, essentially all are seen in at least one band of IRAC, most $(\sim 80 \%)$ are detected in MIPS-24, and just $\sim 45 \%$ are seen at MIPS-70. However, $\sim 10 \%$ of these famous, bright objects are saturated in at least one Spitzer band, and many objects do not have IR excesses (or have excesses too weak to be measured at, e.g., $70 \mu \mathrm{m}$ ). Those that are missing flux densities (e.g., without even limits) in Table 4 (or Table 5) are missing because they are off the edge of the covered area, or there is a cosmic ray cluster near the location of the source, corrupting the photometry.

As can be seen in Table 2, out of the 215 previously identified Taurus members, 144 (67\%) are selected in at least one of our color spaces as having a Spitzer IR excess. This is roughly consistent with the two-thirds disk fraction in Taurus. Just 65 $(30 \%)$ are selected in all of our color spaces simultaneously. Out of our 148 new candidate Taurus members, 17 (16\%) are selected in all of our color spaces simultaneously (two of which are likely galaxies, $043349.5+291528$ and $044554.8+240843$, based on spectroscopy). Interestingly, there are a comparable number of non-members (new or previously identified), 21 objects, that are selected in all of our color spaces together.
For the new candidate members, we have an obvious bias in that we cannot find stars without Spitzer IR excesses; note that the sample of previously identified Taurus members includes Taurus members without IR excesses. We also have a bias in that three of these color selections use $24 \mu \mathrm{m}$ in some fashion (either as overall brightness or as part of the color). This selection mechanism biases our sample of YSO candidates toward a high fraction with $24 \mu \mathrm{m}$ detections; see Table 3. Just $\sim 9 \%$ of these objects are not seen at $24 \mu \mathrm{m}$. Moreover, because previous surveys on the whole were using less sensitive instruments, the new potential objects that we have discovered here are on average fainter in the optical and NIR than the sample of previously identified Taurus members (see Table 3 ).

Many objects newly identified here have large IR excesses, but several of them have small excesses at $8 \mu \mathrm{m}$ and either a low excess or no detection at all at longer wavelengths. These objects are the unselected objects (gray dots) in the $K_{\mathrm{s}}$ versus $K_{\mathrm{s}}-$ [24] diagram (Figure 2) that have $K_{\mathrm{s}}-$ [24] near 0 . These objects are included in our list of new candidate Taurus members only if they have more than $4 \sigma$ excess (see Section 3.1.4); we have dropped objects whose apparent $8 \mu \mathrm{m}$ excess is completely inconsistent based on Planck function considerations with a photospheric $24 \mu \mathrm{m}$ measurement (or limit). These $4 \sigma 8 \mu \mathrm{m}$ points seem to be real, in that the distribution of, e.g., [3.6]-[8] colors are near zero for the overwhelming majority of stars in the catalog, and these objects are clearly redder than average. However, without detailed modeling beyond the scope of this paper, it is puzzling how objects could have legitimate, real $4 \sigma$ excesses at $8 \mu \mathrm{m}$ and small $24 \mu \mathrm{m}$ excesses. These objects have low grades of confidence in Table 7, and generally will require additional observations to resolve.

\subsubsection{Additional Information from the Spectroscopy}

Of the 83 stars with optical spectroscopy, not all of them are securely identified members of Taurus. Additional information, such as emission lines which are typical of 1-5 Myr T Tauri stars, can help inform our membership assessment. Twenty-six have $\mathrm{H} \alpha$ in emission at any level. M stars that are not members of Taurus but possess typical levels of stellar activity can also have $\mathrm{H} \alpha$ in emission. Figure 6 shows the $\mathrm{H} \alpha$ equivalent width as a function of spectral type for all stars reported in this work 


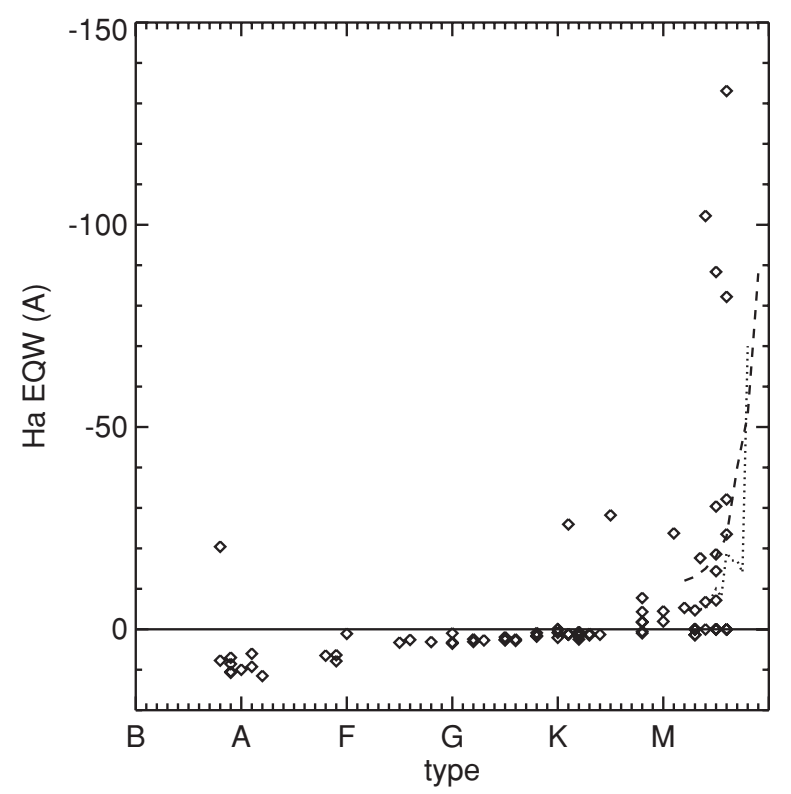

Figure 6. $\mathrm{H} \alpha$ equivalent widths (where values $<0$ indicate emission) as a function of spectral type, for all objects with spectral types reported here (including stars listed solely in Appendix B and dropped as non-members). Note that there is an additional M0 star, not shown, with an $\mathrm{H} \alpha$ equivalent width of $-498 \AA$. The dotted and dashed lines are, respectively, lines from Slesnick et al. (2008) and Barrado y Navascués \& Martín (2003), and indicate the expected quiescent $\mathrm{H} \alpha$ emission from normal stellar activity. We take stars with emission beyond these levels as clear YSOs.

(including those stars listed solely in Appendix B which we dropped as non-members). The lines shown are from Slesnick et al. (2008) and Barrado y Navascués \& Martín (2003), dividing accretion from "normal" activity levels of $\mathrm{H} \alpha$ at the late $\mathrm{M}$ types. Stars for which we have detected a strong IR excess plus an $\mathrm{H} \alpha$ equivalent width in emission larger than the cutoff as a function of spectral type are objects we have placed in the secure "new member" bin-see Section 3.3 and the data tables-except for the Be star which cannot be a member of Taurus (see Appendix B.3). We also list objects that have a more moderate $\mathrm{H} \alpha$ emission but still a very strong IR excess (not Class III but Class I, II, or flat) as secure new members. Objects with moderate or no $\mathrm{H} \alpha$ emission and small IR excess (Class III) need additional indications of membership, such as lithium, radial velocities, proper motions, or high-spectralresolution observations of gravity-sensitive lines.

One way to identify intermediate-gravity objects, e.g., neither giants nor dwarfs but YSO-like gravities, is found in Slesnick et al. (2008) and applies to stars of type M1 and later. It uses the TiO $8465 \AA$ index and $\mathrm{Na} 8190 \AA$ index; this analysis for our qualifying stars is presented in Figure 7. All of our new members except one have gravities in the region of this diagram occupied by previously identified Taurus members; all of the rest of our candidate members shown here are also roughly consistent with YSO gravities, given the scatter in this measurement. These objects are indicated in the notes column of Table 7 . The one new member that may have more giant-like gravity is SST Tau $042920.8+274207$, which may appear in that location in the diagram due to reddening; reddening will push points up and to the left in this figure. This object is particularly perplexing, in that it has many characteristics of youth and also those of the post-main sequence. Our calculation of $A_{V}$ (Section 3.3) suggests that $A_{V} \sim 4$. Additional modeling (beyond the scope of this paper) is needed to further investigate this object. We

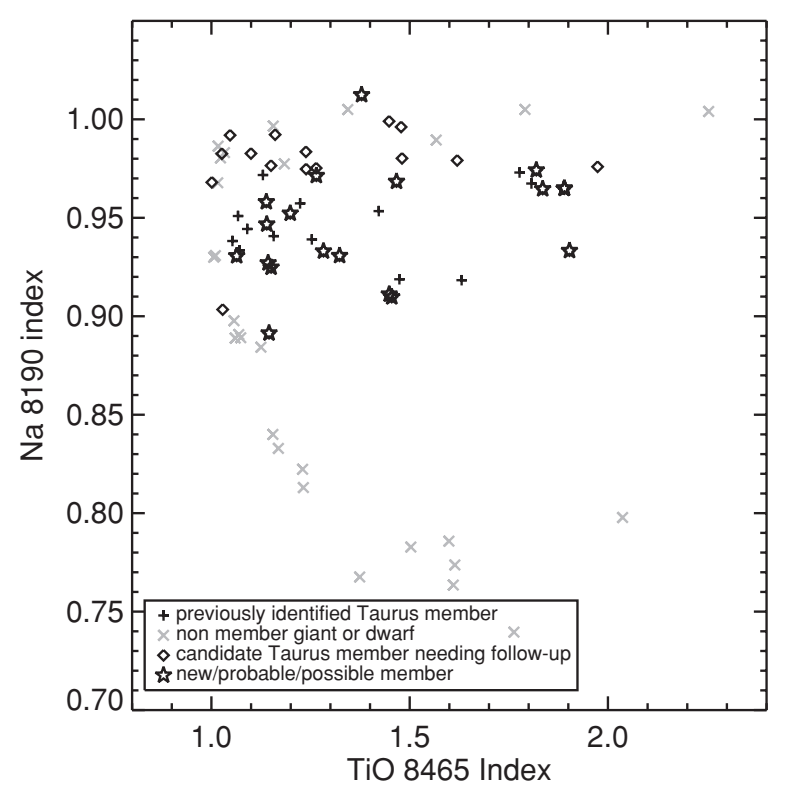

Figure 7. $\mathrm{Na} 8190$ and $\mathrm{TiO} 8465$ indices for the M1 and later stars in our optical spectroscopic sample. Gray $\times$ symbols are objects from Slesnick et al. (2008) that are known giants (large $\mathrm{Na} 8190$ indices) or dwarfs (the remaining points, with smaller $\mathrm{Na} 8190$ indices). The + signs are previously identified Taurus members. The star symbols are new members, probable new members, and possible new members. The diamonds are remaining objects with spectra from the 148-object list of new candidate YSOs, e.g., those objects needing more follow up. All but one of our new members have gravity measurements consistent with YSO gravities, and even that one may be YSO-like (see text).

note here that several of the objects discarded as giants (based on visual examination; see Appendix B.4) would appear in this diagram as having gravities consistent with giant gravities, if they were included in this figure.

\subsubsection{Summary of Properties of All the New Members}

Out of the 148 new candidate Taurus members, according to the letter grades we assigned (Section 3.1.4), there are 50 with letter "A+," "A," or "A-" (most believable), 66 with grade "B+," "B," or "B-," and 25 with grade "C+," "C," or "C-" (least believable). These grades incorporate all the available photometric and spectroscopic information for each object; the remaining seven do not have grades because they are galaxies based on the follow-up spectroscopy. We have spectroscopy for 114 of the 148 objects, 90 of which are optical spectra that have been analyzed. We can report stellar spectral types for $83(\sim 90 \%)$ of those (again, the remaining seven are galaxies). Although we have biased our spectroscopic follow up toward the brighter objects, such a low rate of finding galaxies gives us confidence that our YSO selection process is reasonably successful in that it finds many more stars than galaxies. Note that, while all the "new members" are also grade A objects, not all grade A objects are "new members" because of the need for additional data in many cases. The 83 stellar objects include newly confirmed YSOs and objects that will most likely turn out to be background giants when more data are acquired.

There are 34 new members, 3 probable new members, 10 possible new members, seven extragalactic objects, one other object (Be star), 60 stars needing additional follow-up observations, and 33 pending any follow-up observations. All of the individual SEDs appear in Appendix A. Combining the 34 new members of Taurus, three probable new members, and 10 possible new members yields a total of 47 new objects of various 
shades of confidence. This represents an increase of 20\% (by number) over the previously identified members covered by our map.

Of the remaining 148 objects less the 47 new Taurus members (new, probable new, and possible new), 60 more objects have optical spectra resembling stars, but we need additional data to distinguish these potentially interesting (often transition disk-type SED) objects from background giants or foreground objects (using, e.g., lithium abundances, radial velocities, proper motions, etc.; these are in the "needs additional follow up" category). If they turn out to be non-members, the IR excess we observe needs to be explained. Finally, 33 await additional data beyond the Spitzer photometry to confirm or refute their new Taurus membership status ("pending follow up"). Thus, more new objects could still be in this data set. For completeness, the remaining $148-47$ objects are also listed in Table 8 , sorted by their category, such that the objects we grade as most likely to be new members appear at the top of the list.

\subsection{Detection at Other Bands}

Since we have a wealth of data at other wavelengths, it is possible that we can find some additional evidence for youth for our potential new Taurus members among them. For example, young stars are known to be bright in X-rays and UV. We did in fact include the information below in our ultimate ranking of the objects (see Section 3.1.4).

The XEST fields were optimized to cover the previously identified YSO population, and do not cover our entire Spitzer field. Out of the previously identified YSOs, 107 of 215 are detected in $\mathrm{X}$-rays, and 50 are detected by the XMM-Newton OM (in UV). Out of the entire 148 star list of potential new Taurus members, seven are detected in X-rays $(041940.4+270100=$ XEST-16$024,042215.6+265706=$ XEST-11-078, 042936.0+243555 = XEST-13-010, 043326.2+224529 = XEST-17-036, 043456.9+ $225835=$ XEST-08-003, 043542.0+225222 = XEST-08-033, and $044125.7+254349=\mathrm{XEST}-07-032)$, and two are also detected by the XMM-Newton OM $(042215.6+265706=$ XEST$11-078=$ XEST-11-OM-122 and 042936.0+243555 = XEST$13-010=$ XEST-13-OM-002). These objects are noted in Table 7. The two objects detected in both X-rays and the XMMNewton OM are already confirmed new members. Of the remaining objects detected in X-rays, there are three more confirmed new members, one probable, one possible, and two pending additional follow-up; five of them appear in Scelsi et al. (2007) as potential members (see Table 7).

The SDSS stripes do not cover our entire field either, but they provide $u$-band observations for those regions they do cover; however, extinction strongly affects the numbers of objects detected. Out of the 215 previously identified Taurus members, 109 are detected at SDSS $u$; out of the 148 stars we list as potential new members, 63 are detected at SDSS $u$ (18 of which are confirmed new members). Out of those objects, 33 have apparent UV excesses above the locus formed by all of the objects in our catalog with Sloan uriz photometry (17 of these 33 are confirmed, probable, or possible new members). These objects are noted in Table 7.

\subsection{Locations of New Candidate Taurus Members}

As can be seen in Table 8, many of the new objects are quite close to previously identified Taurus objects. Figure 8 shows the projected location of the sample of previously identified YSOs, the new YSO candidates we have selected, and those new YSO candidates with the results of the spectroscopy folded in. Because one of the goals of this project is to look for widely distributed Taurus members, we did not restrict our search for new objects to the regions already occupied by Taurus members. While proximity to previously identified YSOs was a component in our ranking scheme, it was only one of many criteria (Section 3.1.4). As can be seen in Figure 8, the new candidate objects are generally more isotropically distributed than the previously identified members. However, most of the new Taurus members tend to be found near the previously identified Taurus members. There is a new loose grouping of YSOs found near $(\alpha, \delta)=(70,24)$. Further spectroscopic results are needed to complete our search for an extended population of YSOs in Taurus.

\subsection{Spectral Types and YSO Classes}

Figure 9 shows a histogram of the spectral types of the previously identified Taurus members plus the 34 confirmed new objects; most of the new objects are M spectral typesone is "early K," one is a K5e, four are K7-M0, and the rest are $\mathrm{M}$ stars. Of the probable new members, there are one $\mathrm{M}$ star, one A star, and one "<M0"; of the possible new members, there are two M stars, and the rest are K4 or earlier (one late F, two $\mathrm{G}$ stars, five $\mathrm{K}$ stars). These earlier types are less secure new members because they have small IR excesses and/or weak $\mathrm{H} \alpha$.

Most of the 47 new objects (32 of them) are YSO Class II. Figure 9 shows a histogram of the types of the previously identified objects plus the 32 confirmed new objects. There are five "flat" YSOs in the 32 new objects, one Class I, and four Class IIIs; the rest are Class II.

\subsection{Ensemble SED Properties of Currently Identified Taurus Members}

Having established the location and spectral type distribution of the new members relative to the established members, we now investigate the SED properties of the ensemble. Figure 10 shows representations of the SEDs for all of the 215 previously identified Taurus members with $K_{\mathrm{s}}$ detections (on the left), and the 34 confirmed members discovered using the Spitzer data (on the right). Note that (a) these SEDs are normalized to $K_{\mathrm{s}}$ (objects missing $K_{\mathrm{s}}$ due to saturation are not included, so a total of 211 previously identified Taurus members are shown), and (b) the SEDs appear individually in the appendix below, see Appendix A. We have normalized to $K_{\mathrm{s}}$ because we wished to investigate the variation of the shapes of the SEDs, not their relative intrinsic brightness and/or extinction; $K_{\text {s }}$ provides a value available for most objects (see Table 3) and is an admittedly imperfect compromise between disk and photosphere emission. For each wavelength, there are very broad, non-Gaussian distributions of points. In the optical in particular, but also at wavelengths at least as long as $24 \mu \mathrm{m}$ (see, e.g., Bary et al. 2007), the intrinsic variability of the objects can contribute significantly to the scatter.

The figure uses box plots; these box plots have been used in other papers (e.g., Rebull et al. 2006a; Flaccomio et al. 2003) as a mechanism for interpreting scatter plots. For each of the wavelength points, the boxes capture the median and the first and third quartiles of the distribution in $\lambda F_{\lambda}$ (including measurements only, not limits). The lines extend to the most extreme values that are not more than 1.5 times the interquartile range, and the circles are those points outside 1.5 times the 

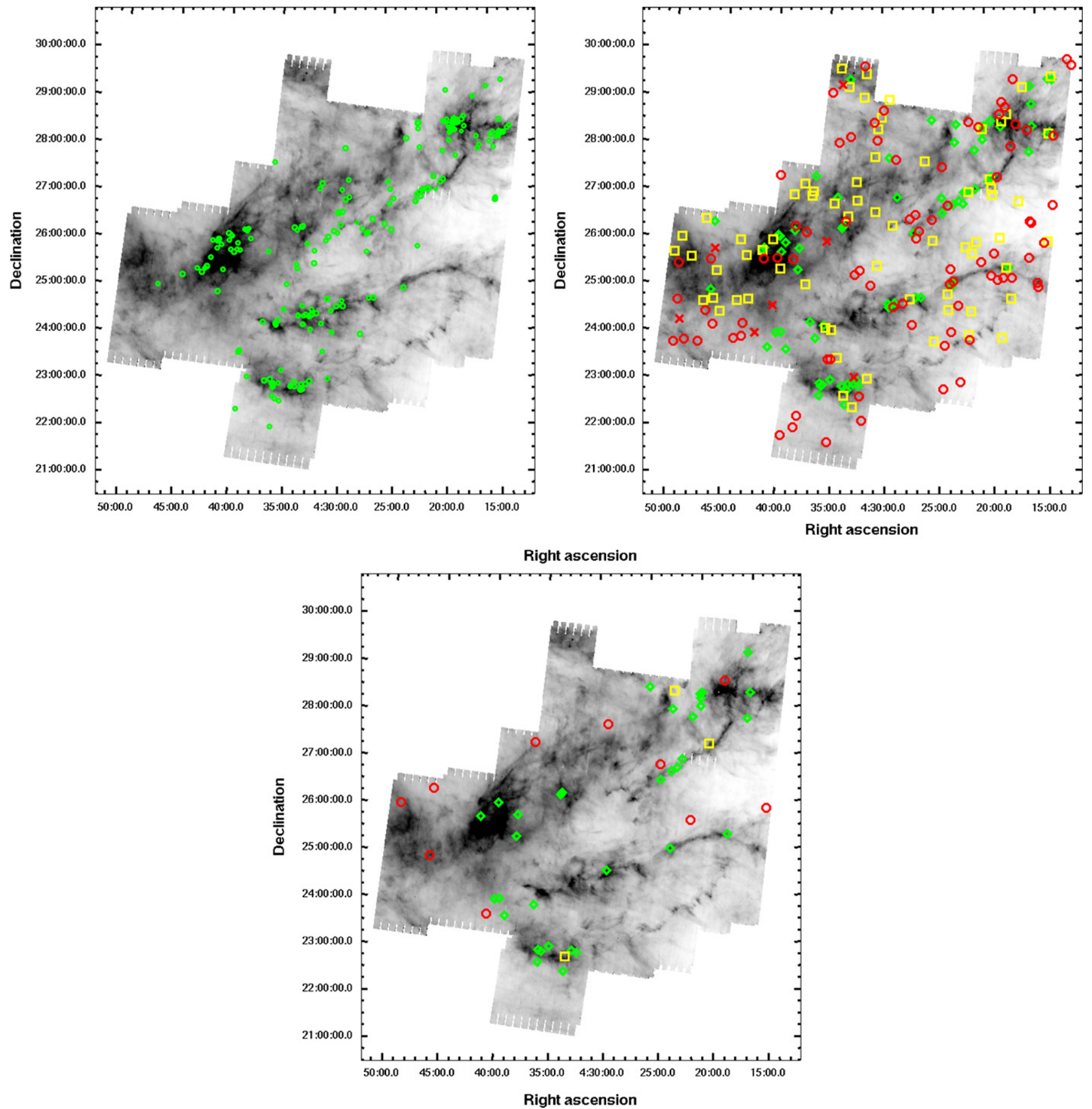

Figure 8. Physical location of various samples of Taurus objects on the $160 \mu \mathrm{m}$ image (reverse grayscale): (top left) previously identified Taurus members (green circles), which tend to follow $160 \mu \mathrm{m}$ emission; (top right) all new candidate objects: green diamonds $=$ grade A, yellow boxes $=$ grade B, red circles $=$ grade C, $x=$ extragalactic; (bottom left) the 47 new Taurus objects: green diamonds $=$ new members, yellow boxes $=$ probable new members, red circles $=$ possible new members. The confirmed new Taurus members thus far tend to be found near the previously identified Taurus objects.

interquartile range. For cases like those found in, e.g., Rebull et al. (2006a), there are upper limits in the distribution, and the Kaplan-Meier (K-M) estimator for censored data can be used to take into account the upper limits present in the data. However, in this present case, for each of the Spitzer points, we have both upper and lower limits in the distribution, so the K-M estimator fails. The offset gray boxes in Figure 10 use all of the upper and lower limits as real detections at the location of the limit. The influence of the large number of limits can particularly be seen at MIPS bands, where the lower edges of the box are substantially lower with the limits included as real detections than without. There are no detections at $160 \mu \mathrm{m}$ among the 34 new member stars, so only limits can be used.

The solid line near the medians is the "median Taurus SED" from D'Alessio et al. (1999). It is clear that we have not compensated for reddening in the optical bands, as the D'Alessio SED is significantly above our medians at blue wavelengths (shorter than $1 \mu \mathrm{m}$ ). (D'Alessio et al. individually dereddened the SEDs before combining them to get the median; we would need to apply a reddening of about $A_{\mathrm{J}}=0.8$ to the D'Alessio 

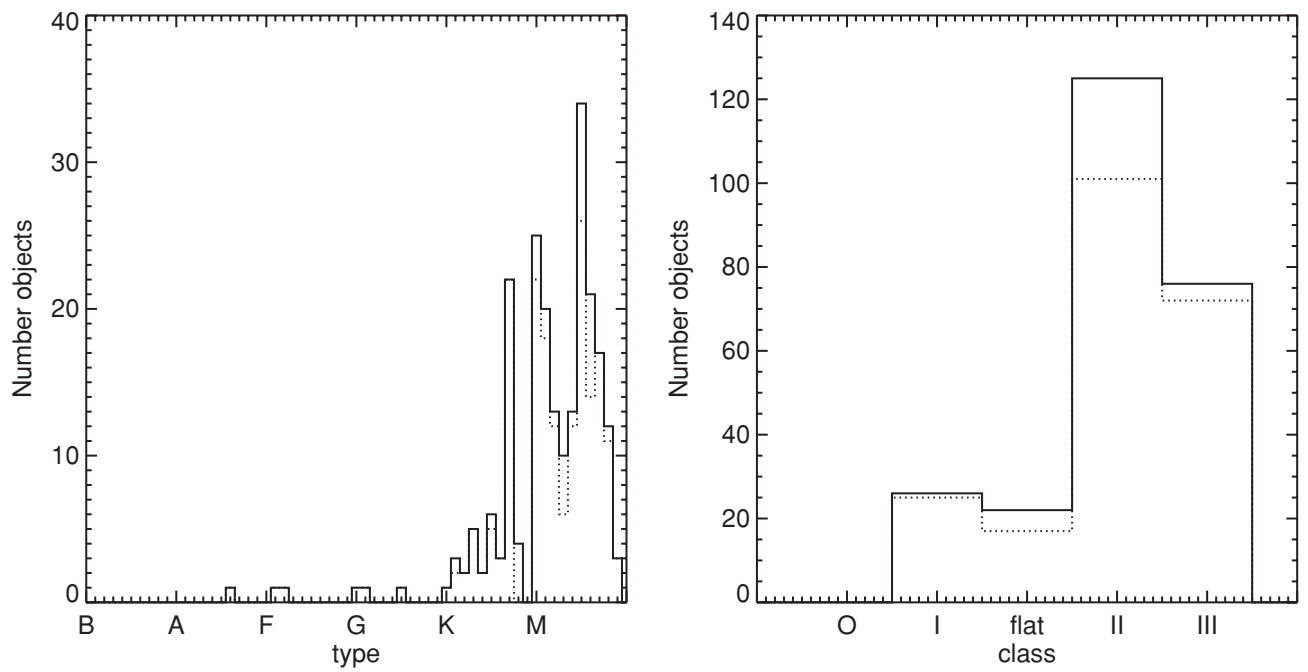

Figure 9. Left: histogram of types for all previously identified members with spectral types plus new confident members (solid line) and just the previously identified members (dotted line). Right: histogram of all the previously identified members plus new confident members (solid line) and just the previously identified members (dotted line).

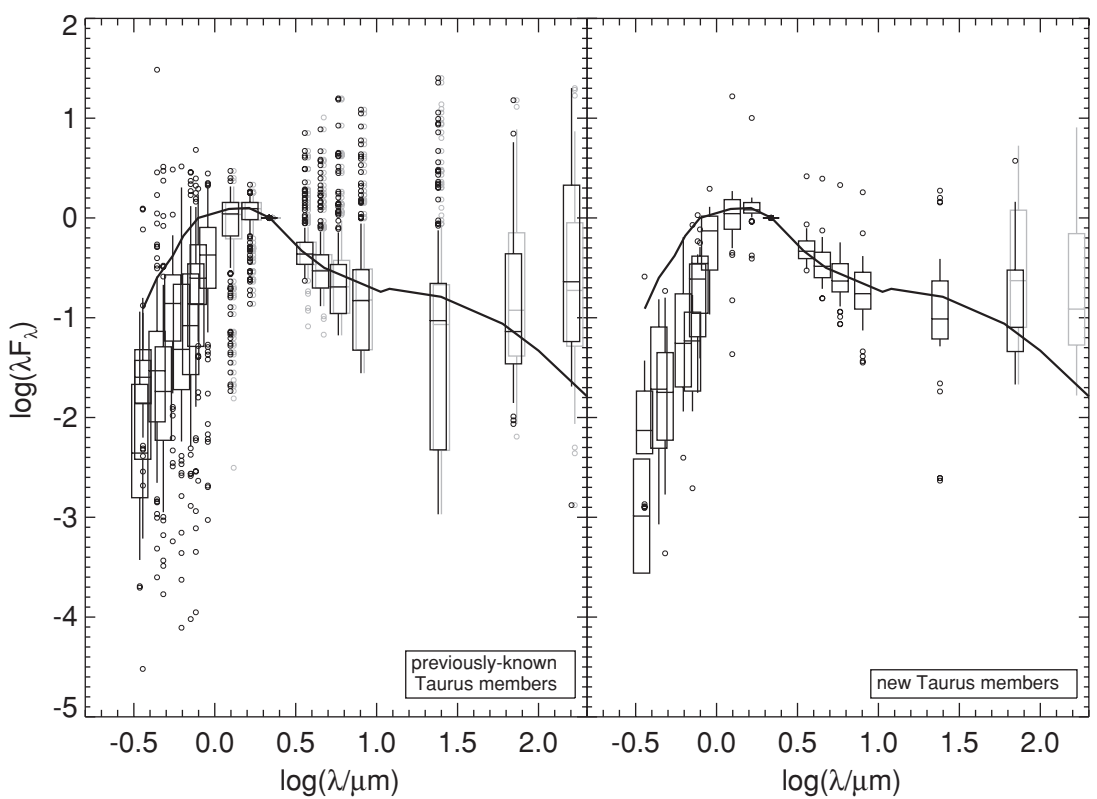

Figure 10. Box plot (see text) of all SEDs, normalized to $K_{\mathrm{s}}$, for (left) the 211 (out of 215 possible) previously identified Taurus members having a $K_{\mathrm{s}}$ measurement, and (right) the 34 (confirmed) new members discovered using the Spitzer data. The central line in each box denotes the median; the ends of each box are the first and third quartile of the distribution; the lines extend to the most extreme values that are not more than 1.5 times the interquartile range; and the circles are those points outside 1.5 times the interquartile range. The gray, slightly offset boxes are the same representation, but treating all of the upper and lower limits as real detections at that limit point; see text for more discussion. (Note that none of the new members have a detection at $160 \mu \mathrm{m}$, so that wavelength has only a gray box.) The solid line near the medians is the "median Taurus SED” from D' Alessio et al. (1999).

median SED to make it match our median SED.) The D'Alessio SED tracks the rest of our medians reasonably closely, although it must be noted that our medians include all of the Taurus members, not just the K5-M2s, and not just the stars with IR excesses. Our medians are slightly below the D'Alessio SED at $4.5-8 \mu \mathrm{m}$, more below the D'Alessio SED at $24 \mu \mathrm{m}$, then on the D'Alessio SED at $70 \mu \mathrm{m}$, then above it at $160 \mu \mathrm{m}$; in all cases, the medians match within the box, e.g., between the first and third quartiles. Since there are more lower than upper limits at $24 \mu \mathrm{m}$, the "true" median is likely to be lower still, as can be seen by the location of the gray median line. Similarly, the "true" median at 70 and $160 \mu \mathrm{m}$ is likely to be lower than our calculated value. At $160 \mu \mathrm{m}$ in particular, we are likely seeing the effects of sensitivity; even our upper limits are reasonably shallow, and the location of the gray median line is closer to the D'Alessio SED. The medians at each band of the 34 new members are not much different than the medians of the previously identified members, although they are slightly brighter. We are unable to find stars without IR excesses using Spitzer selection criteria, so we expect our new objects to have larger excesses on average than the entire ensemble of Taurus objects. At $70 \mu \mathrm{m}$, the new objects are as a whole much brighter than the previously identified sample. At $24 \mu \mathrm{m}$, the median is reasonably comparable but the lower boundary to the box (e.g., location of first quartile) is much brighter for the new objects than for the previously identified sample. Both of these effects are undoubtedly a result of our slight MIPS bias in source selection (see Section 4.1). 

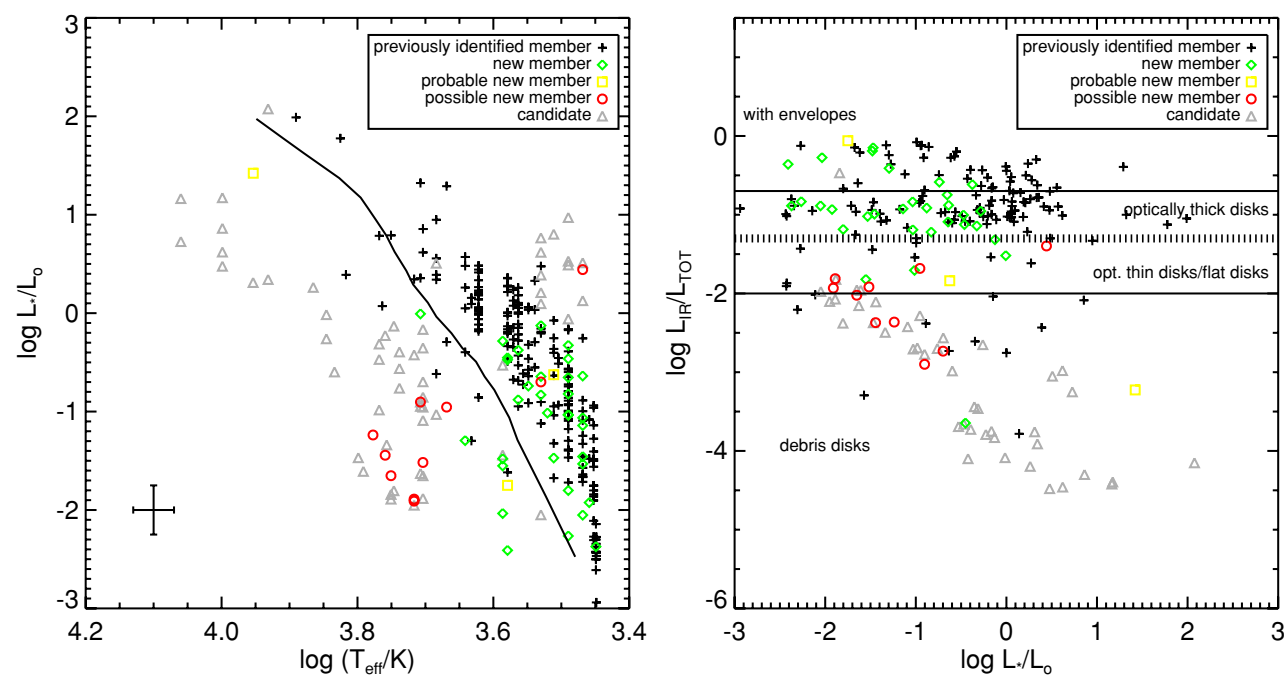

Figure 11. Left: HR diagram for the previously identified members (+), the new members (green diamonds), the probable new members (yellow squares), the possible new members (red circles), and the rest of the candidate members (gray triangles), using the $L_{*}$ values calculated (and $T_{\text {eff }}$ values assumed) in the process of calculating $L_{\mathrm{IR}} / L_{\text {total }}$; see text for caveats. Typical error bars are indicated in the lower left; the error in temperature will be larger for earlier types. The ZAMS from Siess et al. (2000) for the Taurus distance is overplotted as a solid line for reference. Most of the highest confidence new members have positions in this diagram quite consistent with the positions of previously identified Taurus members. All three of the probable new members and just two of the possible new members also have consistent positions. The remaining possible new members are apparently underluminous; see text. Many of the candidates awaiting further data also have positions consistent with Taurus members, but many are also apparently subluminous. Right: plot of $L_{\mathrm{IR}} / L_{\text {total }}$ against $L_{*} / L_{\odot}$, with the same notation as the left figure. Approximate dividing lines for the interpretation of $L_{\mathrm{IR}} / L_{\text {total }}$ are indicated. The dividing line between optically thick disks and disks that are either optically thin or flat is hatched to indicate that it is a "fuzzy" dividing line.

\subsection{Ensemble $L_{\mathrm{IR}} / L_{\mathrm{total}}$ Properties of Currently Identified Taurus Members}

We calculated $L_{\mathrm{IR}} / L_{\text {total }}$ for the previously identified and new candidate Taurus members; see Section 3.3 and Tables 6 and 7. Since $L_{*}$ is a by-product of this calculation, we present an HR diagram in Figure 11. The $T_{\text {eff }}$ values appear quantized because the $T_{\text {eff }}$ value for each star was assumed based on its spectral type. The new members, probable new members, and possible new members are indicated separately on this diagram, along with the previously identified and pending samples. Most of the highest confidence new members have positions in this diagram quite consistent with the positions of previously identified Taurus members. All three of the probable new members and just two of the possible new members also have consistent positions. Both the previously identified and new groups have some apparently subluminous members, with additional such objects among the sample awaiting more data. These objects could be subluminous due to being, e.g., edgeon disks, or they might not be members. Further investigation is warranted. Many of the candidates awaiting additional data and/or analysis fall in the pre-main-sequence regime of this diagram, but many do not; these are likely to turn out to be background dusty giants. There seems to be a loose clump of new objects near $\log T_{\text {eff }} \sim 3.75, \log L_{*} / L_{\odot} \sim-1.8$, and many of the earlier-type objects still awaiting additional data are also considerably too faint. These objects are probably unlikely to be Taurus members-they could be background debris disk candidates-and are indicated individually in the data tables. We included the position of the object in the HR diagram in our individual assessment and grading of each object (Section 3.1.4).

Figure 11 also shows the $L_{\mathrm{IR}} / L_{\text {total }}$ versus $\log L_{*} / L_{\odot}$ for the sample. Due to Spitzer's superior sensitivity, resolution, and wavelength coverage versus IRAS, the $L_{\mathrm{IR}} / L_{\text {total }}$ values here are likely to be more reliable than those values presented in, e.g., Cohen et al. (1989). Systems with $L_{\mathrm{IR}} / L_{\text {total }}>0.2$ (e.g., GV Tau AB) are expected to be disk systems with circumstellar envelopes, and indeed most of the systems in this range show rising or flat SEDs. Systems with $L_{\mathrm{IR}} / L_{\text {total }}$ of $0.05-0.2$ (e.g., DL Tau, CY Tau) are expected to be optically thick disks lacking envelopes, with large values indicating more flared disks. Most of the known T Tauri stars in the cloud fall in this range. Systems with $L_{\mathrm{IR}} / L_{\text {total }}$ near 0.01 (e.g., V410 Anon 24) are either optically thick disks highly flattened by dust settling, or systems just becoming optically thin as they transition to debris disks. Finally, systems with $L_{\mathrm{IR}} / L_{\text {total }}<0.01$ (e.g., V819 Tau) are similar to classical debris disks. Approximately 15 of these are found in the survey, among the previously identified members combined with the new members. Most of the previously known and new objects have substantial disks or envelopes. Most of the least confident members (and most of those awaiting more data) appear to have very tenuous disks. Certainly some legitimate members appear at low $L_{\mathrm{IR}} / L_{\text {total }}$ as well, so some of these candidates may be legitimate.

\subsection{Comparison to Other Selection Methods}

Thus far, our spectroscopic follow-up suggests very few galaxies are among our YSO candidates. While we have not obtained spectra of every candidate, and our spectroscopic follow-up has generally been of the brighter candidates, it is still important to note that our screening does seem to successfully weed out galaxies.

As mentioned above, there are many discussions in the literature also seeking to identify YSOs using Spitzer color selections (e.g., Allen et al. 2004; Padgett et al. 2008b; Rebull et al. 2007; Harvey et al. 2007; Gutermuth et al. 2008). In this section, we compare our color selection to two other popular color selection mechanisms. Harvey et al. (2007) describes the c2d/Gould's Belt method, which works with large-scale maps of star-forming regions similar to that for Taurus. With the mitigation of extragalactic contamination as a primary goal, Harvey et al. (2007) apply several color cuts and use shape/ fitting information from the $\mathrm{c} 2 \mathrm{~d}$ pipeline, combined with manual 
Table 9

Comparison of Selection Methods I

\begin{tabular}{|c|c|c|c|}
\hline Property & This Work & Harvey et al. (2007) Criteria & Gutermuth et al. (2008) Criteria \\
\hline \multicolumn{4}{|c|}{ Of entire catalog } \\
\hline Part that can be considered & $122616(18 \%)$ & $7119(1 \%)$ & $\gtrsim 89003(13 \%)^{\mathrm{b}}$ \\
\hline Number of stars & $98476^{\mathrm{a}}$ & 965 & 74254 \\
\hline Number of galaxies or other contaminants & $80995^{\mathrm{a}}$ & 4644 & 14176 \\
\hline Number of YSOs & $870^{\mathrm{c}}$ & 1510 & 573 \\
\hline \multicolumn{4}{|c|}{ Of previously identified members } \\
\hline Part that can be considered & $206(96 \%)$ & $154(72 \%)$ & $\gtrsim 187(87 \%)$ \\
\hline Number of stars & $172^{\mathrm{a}}$ & 6 & 82 \\
\hline Number of galaxies or other contaminants & $88^{\mathrm{a}}$ & 7 & 2 \\
\hline Number of YSOs & 144 & 141 & 103 \\
\hline \multicolumn{4}{|c|}{ Of sample newly identified here } \\
\hline Part that can be considered & $148(100 \%)$ & $128(86 \%)$ & $\gtrsim 141(95 \%)$ \\
\hline Number of stars & $87^{\mathrm{a}}$ & 0 & 76 \\
\hline Number of galaxies or other contaminants & $116^{\mathrm{a}}$ & 28 & 5 \\
\hline Number of YSOs & 148 & 100 & 60 \\
\hline
\end{tabular}

Notes.

${ }^{a}$ This number of objects meets the star or galaxy criteria in any of the color-color or color-magnitude spaces used. Thus, total number of objects is not equal to number stars+number galaxies+number YSOs. See text.

b This method starts with a sample detected at all four IRAC bands (89,003 objects) and semi-manually adds in additional objects (see text). The largest sample of those potential additional objects are those seen at $J H K_{\mathrm{S}}[3.6]$ [4.5], which in this Taurus data set is 76,522 more objects, so $\gtrsim 165525=89003+76522$ might be reported here instead to indicate this semi-manual addition of objects. However, there is no easy method to take those additional objects and break them into stars and galaxies, so the rest of this section of the table is taken out of 89003 objects.

${ }^{c}$ This number objects meets the YSO criteria in any of the color spaces investigated. After the imaging, etc. tests, this 870 number is reduced to 148 new candidate YSOs +144 previously identified YSOs $=292$.

Table 10

Comparison of Selection Methods II

\begin{tabular}{lccc}
\hline \hline \multicolumn{1}{c}{ Parameter Space } & Used by Us & Used by Harvey et al. (2007) & Used by Gutermuth et al. (2008) \\
\hline$[24] /[24]-[70]$ & Yes & $\ldots$ & $\ldots$ \\
$K_{\mathrm{s}} / K_{\mathrm{s}}-[24]$ & Yes & $\ldots$ & $\ldots$ \\
{$[8] /[8]-[24]$} & Yes & $\ldots$ & $\ldots$ \\
{$[4.5] /[4.5]-[8]$} & Yes & Yes & Yes \\
{$[3.6]-[4.5] /[5.8]-[8]$} & Yes & $\ldots$ & $\ldots$ \\
{$[24] /[8]-[24]$} & $\ldots$ & Yes & $\ldots$ \\
{$[24] /[4.5]-[8]$} & $\ldots$ & Yes & $\ldots$ \\
$H-K_{\mathrm{s}} / K_{\mathrm{s}}-[4.5]$ & $\ldots$ & Yes & Yes \\
{$[4.5]-[5.8] /[5.8]-[8]$} & $\ldots$ & $\ldots$ & Yes \\
{$[3.6]-[5.8] /[4.5]-[8]$} & $\ldots$ & $\ldots$ & Yes \\
{$[3.6]-[4.5] /[4.5]-[5.8]$} & $\ldots$ & $\ldots$ & Yes \\
$K_{\mathrm{S}}-[3.6] /[3.6]-[4.5]$ & $\ldots$ & $\ldots$ & Yes \\
$J-H / H-K_{\mathrm{s}}$ & $\ldots$ & $\ldots$ & For measuring $E\left(H-K_{S}\right)$ when no $J$ \\
$H-K_{\mathrm{s}} /[3.6]-[4.5]$ & $\ldots$ & $\ldots$ & Yes \\
{$[4.5]-[5.8] /[5.8]-[24]$} & $\ldots$ & $\ldots$ &
\end{tabular}

examination of the Spitzer images. The Gutermuth et al. (2008) criteria are being used by several different groups, including the IRAC GTO team, and are designed to find YSOs despite a wide variation of extinction values and nebulosity found in star-forming clusters within $1 \mathrm{kpc}$. We note that this method has been recently updated in Gutermuth et al. (2009), though the changes to the method are considered relatively small by the authors, and are not implemented here.

In this section, we have the goal of determining whether we can directly compare, say, the "yield" of this survey with those from other star-forming regions studied by other groups. We conclude that it is not at all straightforward, and direct comparison may not actually be possible because the selection methods are so different. Tables 9 and 10 make an attempt to compare the various methods as we have implemented them, with all the caveats discussed below, but just as it is not easy to compare the "yield," it is not necessarily easy even to compare the methods on a precisely even footing. Note that in order to understand the assumptions that have gone into the numbers in these tables (or into the classes reported in Tables 6 and 7), reading the text below is critical.

One of the most significant differences between our data and those used to develop both of the other two methods is the depth of the IRAC Spitzer data that is used as input. With only two $12 \mathrm{~s}$ IRAC frames per position, our data are at least a factor of 2 shorter integration time (at least square root of 2 less sensitive) than the other surveys that were used to construct these other selection methods. On the other hand, Taurus is also much closer than most of the other associations considered by the other projects. Sensitivity to legitimate YSO cluster members is at least comparable, but more faint contaminating sources will affect deeper surveys. 
An important difference between our selection mechanism and both of the other two methods is the use not only just of different color spaces but also of multiple color spaces in serial (the star is a YSO in this one AND that one AND this other one) versus in parallel (the star is a YSO in this one OR that one OR this other one). Table 10 explicitly lists the color parameter spaces used by each method, but the combination of them is complex and the original paper(s) for each should be consulted.

To reiterate, our method uses a combination of color cuts in a series of color spaces (which can be easily applied to other Spitzer data sets), followed by manual inspection of a variety of properties, including notably high-spatial-resolution optical imaging (which may not be easily applied to other Spitzer data sets). We used color spaces in parallel (this OR that) and manually examined those sources that met the YSO color criteria in any space; there are many thousands of objects that were never inspected manually but are still very likely to be stars and similarly many thousands more that are likely to be galaxies based on their colors. Table 9 indicates that we started with 122,616 objects, of which 98,476 had stellarlike colors in at least one of the spaces we investigated. The overwhelming majority of those, $99 \%$, have colors consistent with stars in all the spaces we investigated and were never subject to the scrutiny of the YSO candidates; 513 of these were also selected as possible YSOs in at least one of the color spaces we investigated, and most have been discarded. Similarly, there are 80,995 objects that are classified as galaxies in any of the spaces we investigated, and $99 \%$ of those look like galaxies in all the spaces we investigated; there are 681 that were also selected as possible YSOs in at least one of the color spaces we investigated, and most have been discarded. Note too that the numbers of stars/galaxies reported later in this table for the samples of previously identified YSOs and new YSO candidates are again the sample of objects selected in any diagram; discussion of the sample selected in all diagrams appeared above.

We now compare the other two specific methods with our method in more detail.

\subsubsection{Harvey et al. (2007, c2d) Criteria}

Harvey et al. (2007) describe the criteria used by the c2d team and subsequently the Gould's Belt team. Especially since these results are incorporated into Evans et al. (2009), which uses statistics of the Spitzer-selected sample to determine relative lifetimes of the Class $0 / \mathrm{I} /$ flat/II stages, it would be nice to understand how our sample selection compares so that our data on Taurus can be compared to the regions studied by these other programs.

To reiterate, we used the following parameter spaces to find candidate YSOs: (1) [24] versus [24]-[70], or (2) $K_{\mathrm{S}}$ versus $K_{\mathrm{s}}-$ [24], or (3) [8] versus [8]-[24], or (4) [4.5] versus [4.5]-[8], or (5) [3.6]-[4.5] versus [5.8]-[8] with an additional [3.6] brightness cutoff, combined with (6) optical (SDSS / CFHT) imaging plus the additional qualitative criteria mentioned in Section 3.1.4 above. The c2d analysis described in Harvey et al. (2007) used the following parameter spaces to find candidates, assigning a quantitative probability that the object is a galaxy or YSO candidate: (1) [4.5] versus [4.5]-[8], and (2) [24] versus [8]-[24], and (3) [24] versus [4.5]-[8], and (4) (if there was a 2MASS match) $H-K_{\mathrm{s}}$ versus $K_{\mathrm{s}}-$ [4.5], and (5) fitting (and removal) of stars/reddened stars by the c2d pipeline, and (6) shape information gleaned from the $\mathrm{c} 2 \mathrm{~d}$ pipeline, and
(7) manual inspection of the 2MASS+IRAC+MIPS images, and (8) manual addition of previously identified YSOs to the list of YSOs. Differences between the studies include the following: (1) only one CMD is the same between the two teams; (2) the c2d team is using the intersection of all their CMDs, and we are using selection in any one color space; (3) the c2d team is using information obtainable only from their pipeline (shape, star fitting); (4) the c2d team does not have optical imaging (though we both examine the Spitzer imaging); (5) the c2d criteria require detection in all four IRAC bands, and MIPS-24 (they use the 2MASS information if it exists); (6) the c2d pipeline performs PSF-fitting photometry in IRAC and MIPS, and our pipeline does aperture photometry on IRAC data and PRF-fitting photometry on MIPS data. In order to attempt a comparison using the best possible criteria, we can of course impose the same color cuts. We cannot obtain the shape information because we reduced our photometry differently. We can approximate the star fitting by dropping the objects with very small colors in all bands.

Out of the entire $\sim 700,000$ object catalog, these criteria can only be applied to $1 \%$ of the objects (see Table 9) because of the multiband detection requirement. Out of this $1 \%$ of the catalog, which is $\sim 7100$ objects, $21 \%$ are identified as YSO candidates (note that we did not manually examine the images of each of these objects in Spitzer or any other bands, whereas the c2d team would have done so), $65 \%$ are identified as galaxies, and $14 \%$ are identified as stars. Note that this does not mean that a c2d-selected YSO candidate sample has $65 \%$ contamination, but rather that out of the objects in the catalog to which the c2d criteria can be applied, $65 \%$ of these objects are immediately categorized as galaxies. Among our set of previously identified Taurus members, 154 can be classified, but 61 of them (29\%) cannot be classified in this scheme because of missing bands. Seven of them (3\%) are identified as galaxies or other contaminants. Six of them (3\%) are classified as stars (the likely WTTS out of the sample), and 141 (92\%) are identified as YSOs. As intended, this selection mechanism (even as we have implemented it) is strongly biased toward YSOs. This sample of previously known YSOs cannot tell us about the contamination rate, but it can tell us about the fraction of objects that might be missed; a c2d-based YSO selection operating on this sample would miss $\sim 5 \%$ of the YSOs with detections in all the requisite bands and IR excesses. The c2d classifications for these previously identified members are listed in Table 6; the same information for the new candidate members is in Table 7. Among our new candidate objects, perhaps unsurprisingly, there is a higher fraction of contaminants. The multiband detection requirement means that $14 \%$ ( 20 objects) cannot be classified. Among the remainder of the sample, there are 28 (19\%) likely galaxies (or other contaminants), no stars (which makes sense because we are only selecting objects with IR excesses), and $100(68 \%)$ likely YSOs in this attempt to use the c2d criteria on our sample. Happily, the YSO candidates compose the largest fraction of our sample.

The c2d criteria require selection in each of the Spitzer-based CMDs or CCDs; our selection requires selection in just one of the CMDs or CCDs. We have noted above in Section 4.1 the effects of instead requiring selection in each of our CMDs or CCDs. We note again here that, aside from the previously identified members, the number of new potential members is comparable to the contaminant hit rate. The $\mathrm{c} 2 \mathrm{~d}$ criteria require a $24 \mu \mathrm{m}$ detection and we are strongly biased toward objects with a $24 \mu \mathrm{m}$ detection, so this is perhaps not surprising. 


\subsubsection{Gutermuth et al. (2008) Criteria}

Gutermuth et al. (2008), in their Section 4.1 and appendix, describe a multistep weeding process. They require detection in all four IRAC bands for most of the process, although they allow for semi-manual addition of objects missing some bands. The number of sources that can be considered as input for this process which appears in Table 9 attempts to represent this semi-manual addition; there are 89,003 objects seen at all four IRAC bands, and 76,522 that are seen at $\mathrm{JH} K_{\mathrm{s}}$ [3.6][4.5] but not [5.8] or [8], and these two components represent the bulk of the sources that can be considered. Additional provision is made in this method for bright, red objects detected at [24] but not all four IRAC bands, and for stars with $24 \mu \mathrm{m}$ excess; since this represents addition of a few thousand sources to the 165,525 sources already considered, $\mathrm{a} \gtrsim$ symbol is used in the table. The Gutermuth method also works in dereddened colors for some criteria, requiring a high-spatial-resolution $A_{V}$ map, which we do not have and thus cannot implement exactly in a parallel fashion. We note too that the Gutermuth et al. criteria relies mostly on the [4.5]-[5.8] color to avoid effects of reddening that may be found at $3.6 \mu \mathrm{m}$. Since the overall median $A_{V}$ toward Taurus is low $\left(A_{V} \sim 3\right.$, which has a very small effect on $3.6 \mu \mathrm{m}$ ), we did not deredden our colors, and simply applied the Gutermuth et al. criteria to our observed colors. We did not semimanually add objects to the list following their Section 4.2, and we added a few criteria to remove some blue objects found in our catalog; to their "embedded" criteria, we added an additional [5.8]-[8] $>-0.5$ and [3.6] $-[4.5]>-0.5$, and to their "Class II" criteria, we added [3.6] $-[4.5]>-0.5$. Two of the many color parameter spaces that Gutermuth et al. use are the same as ours; see Table 10.

Differences between the Gutermuth criteria and ours include the following: (1) only two CMDs are the same between the methods, (2) the Gutermuth method sometimes uses the intersection of the color spaces (like c2d) and sometimes takes selection in just one color space as sufficient (like us), (3) the Gutermuth method provides for a semi-manual addition of likely YSO objects, based on color cuts, (4) the Gutermuth method does not require intensive manual examination of multiband images, (5) the Gutermuth method, though multistep, can be applied to any Spitzer+2MASS catalog of a star-forming region, and is not dependent on products of pipelines or the presence of ancillary data (modulo reddening corrections), and (6) the Gutermuth method, as properly applied, needs an $A_{V}$ map and needs to work in dereddened colors. In order to attempt a comparison using the best possible criteria, we have imposed the same basic color cuts, and, because of the low overall $A_{V}$ toward Taurus, we have continued to work in the observed color space, and not dereddened anything. We have not semi-manually added objects to the YSO candidate list (as per Gutermuth's Phase 2 or Phase 3 ).

Because of the multiband detections necessary, these criteria cannot be applied to most of the entire $\sim 700,000$ object catalog; even with the semi-manual addition of sources allowed for in this method, between $50 \%-75 \%$ of the catalog do not have enough detections to be included (see Table 9), but the process of semi-manual addition of sources is likely to catch most legitimate YSOs in the catalog. The sample with four-band IRAC detections is easiest to handle automatically, and out of this sample, $83 \%$ are dropped as stars, $16 \%$ are dropped as contaminants of any of a variety of kinds, $282(0.3 \%)$ are embedded objects (Class 0/I or flat), and 291 (0.3\%) are Class II objects. Among our set of previously identified Taurus members,
28 of them (13\%) cannot be classified because of missing bands. Just two are dropped as contaminants and $82(38 \%)$ are classified as stars; $15(7 \%)$ are identified as "embedded" and $87(41 \%)$ are identified as Class II. Again, as intended, this selection mechanism (even as we have implemented it) is strongly biased toward YSOs.

The Gutermuth et al. classifications (in more finely grained detail) for the previously known objects are in Table 6 and those for our new candidate members are listed in Table 7. Among our new candidate objects, 95\% can be considered, $76(51 \%)$ are classified as stars, $5(3 \%)$ are contaminants, and $60(41 \%)$ are YSO candidates ( 21 objects are identified as embedded and 39 are identified as Class II). There is a slightly higher fraction of contaminants in the new sample than among the previously identified sample. We note that the overwhelming majority of objects that we dropped as a result of the review described in Section 3.1.4 were classified as contaminants using the Gutermuth classifications. We also note that the classification that we derive from a fit to all available points between $K_{\mathrm{s}}$ and $24 \mu \mathrm{m}$ is in good agreement with the Gutermuth et al. SED classification except in higher-extinction situations, as expected.

\subsubsection{Conclusions on Different Criteria}

Comparing the different methods is clearly not at all straightforward. The differences are much more than simply different data reduction methods (aperture versus PSF-fitting photometry) or survey depths. The different color spaces that each study investigates, and the different assumptions that are made (e.g., ORring versus ANDing the color selection) can be seen most clearly in the different results when considering the set of previously identified Taurus members. Since not all of the previously identified Taurus members have strong IR excesses, it is not surprising that no Spitzer-based method retrieves all of them. Interestingly, the Gutermuth et al. selection mechanism returns a higher fraction of previously identified members without IR excesses than the $\mathrm{c} 2 \mathrm{~d}$ method.

Direct comparison of the YSOs retrieved in this paper (or over the TMC as a whole) to any other association is not easy because the selection methods are so different. Unfortunately, numbers from this study cannot simply be dropped into tables from these other studies. Even if we were to re-reduce our data in exactly the same way as the other studies, a direct comparison would be difficult.

Even the contaminant rates are hard to compare. Since all of the follow-up data have yet to be acquired for our candidate objects, it is hard to do a final assessment of our contamination rate. Out of the 80 objects with spectra reported here, at least $45 \%$ are new members, and at least $10 \%$ are contaminants. Over $44 \mathrm{deg}^{2}$, including the previously identified plus the new confirmed objects, we recover at least 4.3 YSOs per $\mathrm{deg}^{2}$, and at least 0.18 contaminants per $\mathrm{deg}^{2}$. The $\mathrm{c} 2 \mathrm{~d}$ method was derived primarily using the c2d observations of Serpens (and SWIRE), and Harvey et al. (2007) estimate of order 0-1 contaminants in their $0.85 \mathrm{deg}^{2}$. Scaling this up to our $\sim 44 \mathrm{deg}^{2}$ map, we would then expect $\sim 50$ contaminants in our map using the $\mathrm{c} 2 \mathrm{~d}$ method! Oliveira et al. (2009) carry out follow-up spectroscopy for the c2d Serpens sample, and find higher contamination (25\%) than was originally estimated in Harvey et al. (2007). However, these contaminants are primarily AGB stars, which should not be as much of a contaminant source in the Taurus map, given the lower galactic latitude of Serpens $\left(5^{\circ}\right)$ as compared with Taurus $\left(-15^{\circ}\right)$. Gutermuth et al. (2008) estimate $\sim 1$ contaminant left in their $40^{\prime} \times 30^{\prime}\left(=0.33 \mathrm{deg}^{2}\right)$ map. Scaling up to $44 \mathrm{deg}^{2}$, then 


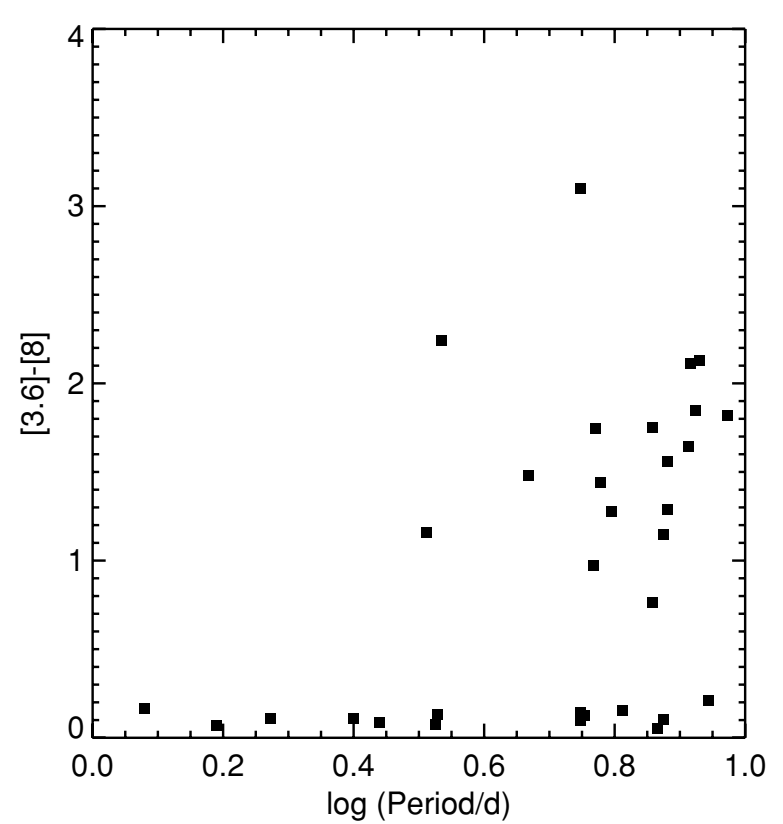

Figure 12. Rotation rates of Taurus stars compared to [3.6]-[8] colors. The relationship seen here, where the slowest rotators are much more likely than the fast rotators to have disks, is consistent with what has been found in other young associations.

we would expect $\sim 130$ contaminants. However, we expect that the actual contamination is much less because this method has been tested on several maps, including larger ones. As more follow-up data are obtained for this and the other associations, a more direct comparison of the complete YSO inventories in these clouds will eventually be possible.

\subsection{Stellar Rotation}

Rebull et al. (2006a) and Cieza \& Baliber (2007) found a correlation between IRAC excesses and rotation rates in Orion and NGC 2264. Taurus is one of the first associations in which rotation of young stars was studied (see, e.g., Edwards et al. 1993), but there are still only 32 stars with measured periods (see Güdel et al. 2007b, and references therein, and Grankin et al. 2008) and IRAC photometry in our catalog. Figure 12 shows the rotation rate against disk excess for those stars. The relationship seen here, where the slowest rotators are much more likely than the fast rotators to have disks, is consistent with what has been found in the other associations.

\section{CONCLUSIONS}

We have presented here Spitzer flux densities for 215 previously identified members of the TMC YSO population. We constructed Spitzer CCDs and CMDs, investigated where the previously identified Taurus members were located, and then used those diagrams to select additional candidate Taurus objects out of a catalog of $\sim 700,000$ objects observed with Spitzer over $44 \mathrm{deg}^{2}$. We used a wealth of supporting data (including ground-based optical imaging) to winnow that list down to 148 new candidate Taurus members. We obtained follow-up optical spectroscopy for about half the sample, thus far finding 34 new members, three probable new members, and 10 possible new members, a potential increase of $15 \%-20 \%$ of young Taurus members. Most of the new members are located in close (projected) proximity to the previously identified Taurus members; most of them are Class II M stars.
In addition to the new members in our sample, there are 60 stars needing additional follow-up observations, and 33 objects pending any follow-up observations at all, so more Taurus members may yet be confirmed out of our list of candidate members. We also found a background Be star, a new planetary nebula, a new carbon star, many background giants, and hundreds of galaxies, just seven of which made it into our final list of 148 YSO candidates.

As part of this project's classification as a Spitzer Legacy Project, enhanced data products have been delivered back to the Spitzer Science Center (SSC), including the catalogs on which this present paper is based.

This study has demonstrated the unique power of Spitzer to efficiently survey large areas of the sky and provide new information on membership and YSO properties even in nearby star-forming regions such as Taurus, which has been extensively studied for decades. Even after many decades of study, our knowledge of membership in Taurus is still incomplete, but definitely improving, thanks to Spitzer.

We thank the Palomar Observatory, Sloan Telescope, CFHT, $X M M-N e w t o n$, and of course Spitzer staff for their assistance using the telescopes. We thank the anonymous referee for thoughtful and thorough comments. This work is based in part on observations made with the Spitzer Space Telescope, which is operated by the Jet Propulsion Laboratory, California Institute of Technology under a contract with NASA. Support for this work was provided by NASA through an award issued by JPL/ Caltech. This research has made use of NASA's Astrophysics Data System (ADS) Abstract Service, and of the SIMBAD database, operated at CDS, Strasbourg, France. This research has made use of data products from the Two Micron All-Sky Survey (2MASS), which is a joint project of the University of Massachusetts and the Infrared Processing and Analysis Center, funded by the National Aeronautics and Space Administration and the National Science Foundation. These data are served by the NASA/IPAC Infrared Science Archive, which is operated by the Jet Propulsion Laboratory, California Institute of Technology, under contract with the National Aeronautics and Space Administration. This research has made use of the Digitized Sky Surveys, which were produced at the Space Telescope Science Institute under U.S. Government grant NAG W-2166. The images of these surveys are based on photographic data obtained using the Oschin Schmidt Telescope on Palomar Mountain and the UK Schmidt Telescope. The plates were processed into the present compressed digital form with the permission of these institutions. This research has made use of observations obtained with XMM-Newton, an ESA science mission with instruments and contributions directly funded by ESA member states and the USA (NASA). The research described in this paper was partially carried out at the Jet Propulsion Laboratory, California Institute of Technology, under contract with the National Aeronautics and Space Administration. M. Audard and C. BaldovinSaavedra acknowledge support from a Swiss National Science Foundation grant (PP002-110504).

\section{APPENDIX A}

\section{SEDS FOR ALL OF THE PREVIOUSLY IDENTIFIED AND NEW CANDIDATE TAURUS MEMBERS}

For each of the previously identified Taurus members, and each of the new candidate members, we provide an SED here in this online-only appendix, Figures 13 and 14, respectively. 


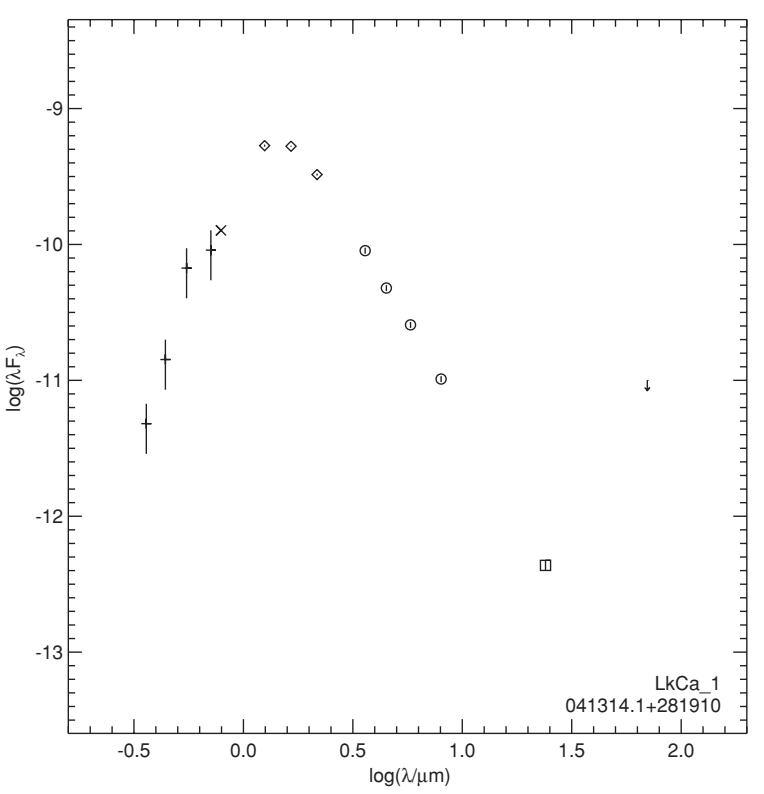

Figure 13. SEDs for all previously identified Taurus members, sorted by R.A. Notation is as follows: triangles-XMM-Newton OM, +-literature Johnson photometry, *-Sloan photometry, $\times$-CFHT photometry, diamonds-2MASS, circles-IRAC, and squares-MIPS. Limits for any band are indicated by arrows. The wavelength is in $\mu \mathrm{m} ; \lambda F_{\lambda}$ is in cgs units $\left(\mathrm{erg} \mathrm{s}^{-1} \mathrm{~cm}^{-2}\right)$.

(The complete figure set (215 images) is available in the online journal.)

Notation is as follows: triangles-XMM-Newton OM, +literature Johnson photometry, *-Sloan photometry, $\times-\mathrm{CFHT}$ photometry, diamonds-2MASS, circles-IRAC, and squaresMIPS. Limits for any band are indicated by arrows. The wavelength is in $\mu \mathrm{m} ; \lambda F_{\lambda}$ is in cgs units ( $\mathrm{erg} \mathrm{s}^{-1} \mathrm{~cm}^{-2}$ ). Each plot has the SST Tau catalog number and, if relevant, a more common name.

For reference, the IRAS PSC all-sky completeness limits at $12,25,60$, and $100 \mu \mathrm{m}$ for a region outside of the Galactic plane are $0.4,0.5,0.6$, and $1.0 \mathrm{Jy}$, respectively. These limits would appear in the figure at $\log \lambda F_{\lambda}=-10.00,-10.22,-10.52$, and -10.52 .

\section{APPENDIX B}

\section{COMMENTS ON INDIVIDUAL MISCELLANEOUS NON-YSO OBJECTS WITH YSO-LIKE COLORS}

In the process of conducting our detailed source-by-source examination, we encountered many objects with YSO-like colors that were not YSOs, such as planetary nebulae and carbon stars. In some cases, these objects are new discoveries, or our observations shed new light on the nature of the object.

\section{B.1. Planetary Nebulae}

Based on imaging, we may have discovered a planetary nebula: SST Tau 041936.1+271731. It appears bright at 24 and $70 \mu \mathrm{m}$, but CFHT imaging reveals a circularly symmetric structure that strongly suggests a planetary nebula. We have not yet obtained follow-up spectroscopy.

PN G174.2-14.6 (=043723.4+250242) is a well-studied planetary nebula (55 references are given in SIMBAD). It appears to have YSO-like colors in [24] versus [24]-[70], [8] versus [8]-[24], [4.5] versus [4.5]-[8] (although it appears as faint enough to likely be a galaxy here), and the IRAC CCD. We ruled it out as a YSO candidate based on the literature early on in our weeding process.

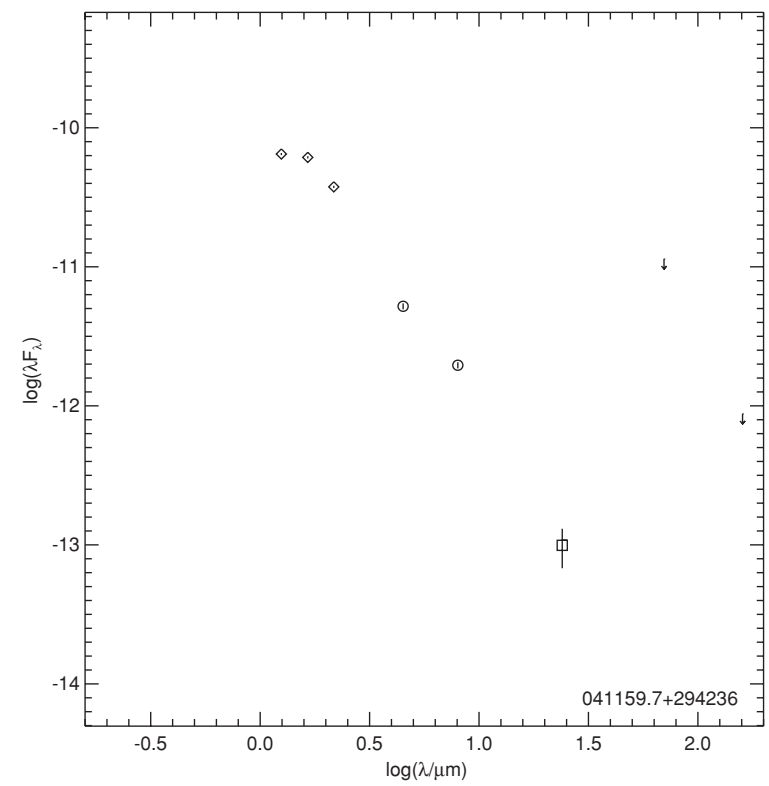

Figure 14. SEDs for all new candidate Taurus members, sorted by R.A. Notation is as in previous figure, and is as follows: triangles-XMM-Newton OM, + +literature Johnson photometry, *-Sloan photometry, $\times$-CFHT photometry, diamonds-2MASS, circles-IRAC, and squares-MIPS. Limits for any band are indicated by arrows. The wavelength is in $\mu \mathrm{m} ; \lambda F_{\lambda}$ is in cgs units (erg s${ }^{-1} \mathrm{~cm}^{-2}$ ).

(The complete figure set (148 images) is available in the online journal.)

\section{B.2. Carbon Stars}

$C^{*} 228(043748.2+254926)$ is a previously identified carbon star. It has YSO-like colors in [24] versus [24]-[70] and [8] versus [8]-[24]. We ruled it out as a YSO candidate based on the literature early on in our weeding process.

Two of the objects we selected initially as YSO candidates turned out to be carbon stars when we obtained spectra: $043250.3+294239$ (= IRAS 04296+2936) and $042818.5+253140$ (= V414 Tau). IRAS 04296+2936 appears to have no references in SIMBAD and may therefore be a newly discovered carbon star; V414 Tau appears in a handful of publications as a carbon star, including Alksnis et al. (2001). IRAS $04296+2936$ has a significantly reddened spectrum, at least in comparison to V414 Tau.

The 2MASS NIR $J H K_{\mathrm{s}}$ photometry for these objects are 8.02, 6.59, and 5.64 for IRAS $04296+2936,7.28,6.11$, and 5.49 for V414 Tau; $C^{*} 228$ is only unsaturated at $H$ and is $3.38 \mathrm{mag}$. These first two objects both have colors considerably redder than those for carbon giants or dwarfs as appearing in Lowrance et al. (2003), and all three are very much brighter than those in Lowrance et al. (2003). If they are carbon dwarfs, they would have to be considerably closer than Taurus, as in $\sim 10 \mathrm{pc}$. However, they are so red in $J-H$ that they are most likely giants; they are so bright that they are most likely background objects, behind the TMC.

Thus far, we have found three carbon stars with YSO-like colors in this $\sim 44 \mathrm{deg}^{2}$ survey. It is possible, indeed likely, that more carbon stars are in our survey, but have not been identified as such.

\section{B.3. Be Background Giant(s)}

One of the stars we selected as a YSO candidate based on three of our color spaces, $043724.8+270919$ (also known as SV* SVS $1085=$ HD 283751), has H $\alpha$ strongly in emission, and we 
classify it as a B8e. We do not have any optical photometry for it, but its 2 MASS $J H K_{\mathrm{s}}$ are 9.79, 9.55, and $9.30 \mathrm{mag}$, respectively. Using its $K_{\mathrm{s}}$ mag and assuming no reddening (as a worst-case but clearly incorrect scenario), and comparing the observed $K_{\mathrm{s}}$ to that expected for young stars, we calculate a distance of $\sim 700$ pc. It seems to be a Be star well behind the Taurus cloud.

$043939.9+252034(\mathrm{JH} \mathrm{225})$ is listed in the literature as a likely non-member based solely on high proper motions. We find that it is a B9 with substantial $A_{V}$. Its 2 MASS $J H K_{\mathrm{s}}$ are 9.48, 9.01, and $8.73 \mathrm{mag}$, respectively. As we did above, we calculate a distance of $600 \mathrm{pc}$. It is also unlikely to be truly a member of Taurus, but additional follow up is needed. It does not have emission lines.

Since these objects were selected as YSO candidates, flux densities appear in the tables above, and SEDs appear in Appendix A.

\section{B.4. Other Very Bright Objects and Giants}

Table 11 includes several bright objects either assumed or confirmed to be background giants.

Several giants are confirmed in the literature (via spectroscopy) as being giants, but are selected in at least one of our color spaces as being YSO candidates. We ruled these out as YSO candidates based on the literature early on in our weeding process.

Elias 3-6 (SST Tau J042907.6+244350) has often appeared in lists of Taurus cloud members. There has been confusion in the literature about its IRAS association, with a mistaken identification with IRAS $04264+2433$ (the HH 414 jet source) often quoted (e.g., Motte \& André 2001). Elias 3-6 is actually IRAS $04260+2437$. At $K_{\mathrm{s}}=2.85$, it is much brighter than any known Taurus member, is saturated in the Spitzer IRAC bands, and shows a [24]-[70] color of 0.04 mag that is consistent with a bright photosphere. Elias (1978) classifies it as a field M8 III giant with 6 mag of visual extinction. The Spitzer results are fully consistent with Elias' original result that this object is not a Taurus member. We drop it from the list as a background giant.

Several objects listed in Table 11 are very, very bright in our survey, and we have assumed primarily based on brightness (and the fact that they seem to have little legitimate IR excess as far as we can determine) that they are background giants. Several of them were selected in at least one of our color spaces as potential YSOs (given the available photometry points; in many cases, later inspection of these images suggested that they were probably saturated, or at least in the nonlinear regime). In some of those cases, since we wondered if they could be YSOs, we obtained spectra of them; the resultant spectral type is listed in Table 11 for use by the community in future studies.

\section{B.5. Objects with Insignificant IR Excesses for Which We Have Spectra}

Several objects appeared upon early inspection to have significant IR excesses at Spitzer bands, and thus we obtained spectra of them. However, upon re-examination, we determined that the IR excesses we detect were not significantly above the expected photosphere. Since these objects are no longer YSO candidates, they do not appear in tables in the main body of the text above or the SEDs shown above. However, since we obtained spectra for many of them, we still report them here for use by the community. They are listed in Table 11 .

\section{B.6. Extragalactic Objects from Luhman et al.}

Eight objects show up as having YSO-like colors in at least one of the color spaces we investigated and are reported as spectroscopically confirmed non-members by Luhman et al. $(2006,2009 b)$. Each of these objects are ones that we had independently discarded as likely galaxies based on inspection of our optical imaging: 041916.1+275048, $042754.4+242414, \quad 042336.9+252628, \quad 042535.5+245739$, $043027.1+280707, \quad 043401.8+231906, \quad 043502.0+233141$, and $044554.8+240843$. This further demonstrates the critical importance of high-spatial-resolution optical imaging in refining a YSO candidate list selected based on Spitzer data, before using valuable spectroscopic telescope time to refute or confirm stellar nature and youth.

\section{B.7. IRAS $04428+2403$ and Other $8 \mu \mathrm{m}$ Pop-up Objects}

IRAS 04428+2403 (SST Tau 044554.8+240843) appears in Kenyon et al. (2008) listed as a confirmed member of Taurus. However, it appears in two prior papers in the literature, one which identifies it as a galaxy (Chamaraux et al. 1995), and the other which (Kenyon et al. 1994) identifies it as only an unconfirmed Taurus member. By inspection of all our available imaging (prior to identifying it with the literature object), we classified it as a galaxy based on its appearance in the optical images. The SDSS imaging pipeline classified this object as extended. SDSS also obtained a spectrum of this object, and it is a very reddened (due to the Taurus cloud) star-forming galaxy with bright $\mathrm{H} \alpha / \mathrm{N}$ II and $\mathrm{S}$ II at a redshift of 0.077 . Therefore, we drop this object from our list of previously identified members.

The SED for IRAS 04428+2403 (seen in Figure 15) resembles those for a class of objects we found in the process of looking for candidate YSOs. These objects seemed to meet all of our criteria for YSO candidacy (see Section 3.1.4). This population has SEDs that looked like they could be YSO candidates, but where the $8 \mu \mathrm{m}$ point in the SED appears significantly above a line connecting the 5.8 and $24 \mu \mathrm{m}$ points. Several of these objects were targeted for optical spectroscopy, and four of them were actually observed. All of them turned out to be extragalactic objects. Tens if not hundreds of the spatially resolved galaxies in our survey (such as IRAS 04428+2403, but also more obvious resolved galaxies) also have this kind of SED. So, we conclude that even though some of these objects are point sources as far as we can tell, they are extremely likely to be galaxies. An example SED for one of the point-like objects is given in Figure 15. All of the objects that were apparent point sources (e.g., the ones that fooled us) appear in Table 11. The ones that are confirmed extragalactic objects are indicated.

The most likely origin of the $8 \mu \mathrm{m}$ pop-up is the polycyclic aromatic hydrocarbon (PAH) emission feature near $8 \mu \mathrm{m}$. Geers et al. (2009) have found that low-mass young stars almost always lack PAH emission features at $11 \mu \mathrm{m}$ in Spitzer spectra or at $3.3 \mu \mathrm{m}$ in ground-based spectra. Thus, we do not expect faint cloud members to show this feature. However, these features are common in lower luminosity galaxies (Weedman \& Houck 2009). PAH features are seen around luminous YSOs such as the Ae star HD 100546 (Malfait et al. 1998), where strong stellar UV fluxes can excite PAH emission. All of the $8 \mu \mathrm{m}$ pop-up sources detected in the Taurus survey, if located at the cloud distance of $137 \mathrm{pc}$, would have low luminosities strongly inconsistent with an early spectral type. Thus, we conclude that none of the $8 \mu \mathrm{m}$ pop-up sources found in the Taurus survey field are consistent with low-luminosity 
Table 11

Individual Miscellaneous Non-YSO Objects with YSO-like Colors ${ }^{\mathrm{a}}$

\begin{tabular}{|c|c|c|}
\hline SST Tau Number & Other Names & Notes \\
\hline \multicolumn{3}{|c|}{ Planetary nebulae } \\
\hline $041936.1+271731$ & & New object; CFHT imaging suggests PN \\
\hline $043723.4+250242$ & PN G174.2-14.6 & Well-studied PN \\
\hline \multicolumn{3}{|c|}{ Carbon stars } \\
\hline $043748.2+254926$ & $C^{*} 228$ & Well-studied carbon star \\
\hline $042818.5+253140$ & V414 Tau & Well-studied carbon star \\
\hline $043250.3+294239$ & IRAS04296+2936 & New (?) carbon star \\
\hline \multicolumn{3}{|c|}{ Background giants from the literature } \\
\hline $043724.8+270919$ & SV* SVS 1085, HD283751 & New(?) Be background giant \\
\hline $043939.9+252034$ & JH 225 & B9 with substantial $A_{V}$ \\
\hline $042021.2+272102$ & phi Tau & Literature giant \\
\hline $042136.8+282458$ & HD 283570 & Literature giant \\
\hline $043238.9+235825$ & IRAS $04296+2352$ & Literature giant \\
\hline $043858.2+263108$ & Elia 3-14 & Literature giant \\
\hline $043926.9+255259$ & Elia 3-15 & Literature giant \\
\hline $043938.8+261126$ & Elia 3-16 & Literature giant \\
\hline $044057.4+255413$ & [TNS87] 8 & Literature giant \\
\hline $042907.6+244350$ & Elias 3-6 & Background giant; name incorrectly associated with IRAS \\
\hline & & $04260+2437$ and $\mathrm{HH} 414$ \\
\hline \multicolumn{3}{|c|}{ Background giants based on brightness } \\
\hline $041324.4+290722$ & HDS 537 & Bright, assumed to be giant; our type is M5 \\
\hline $041443.5+281708$ & [WSB2007] J041443.5+281708 & Bright, assumed to be giant \\
\hline $041943.4+272056$ & $\ldots$ & Bright, assumed to be giant; our type is M3 \\
\hline $042115.2+272101$ & HD 27482 & Bright, assumed to be giant \\
\hline $042344.1+225753$ & V1142 Tau & Bright, assumed to be giant; our type is M2 \\
\hline $042344.1+225757$ & $\ldots$ & Bright, assumed to be giant \\
\hline $042345.3+234503$ & IRAS $04207+2338$ & Bright, assumed to be giant \\
\hline $042517.3+280440$ & IRAS $04221+2757$ & Bright, assumed to be giant; our type is M5 \\
\hline $042519.3+261701$ & IRAS $04222+2610$ & Bright, assumed to be giant; our type is M4 \\
\hline $042630.0+255344$ & IRAS $04234+2547$ & Bright, assumed to be giant; our type is M6 \\
\hline $042731.3+270958$ & IRAS $04244+2703$ & Bright, assumed to be giant; our type is M9 \\
\hline $042805.4+284433$ & ... & Bright, assumed to be giant; our type is $\mathrm{K} 6$ \\
\hline $042955.3+225857$ & IRAS $04269+2252=2$ MASS $04295531+2258579$ & $\begin{array}{l}\text { Bright, assumed to be giant (also Shenoy et al. object); our type } \\
\text { is M8 }\end{array}$ \\
\hline $043121.1+265842$ & $\ldots$ & $\begin{array}{l}\text { Bright, assumed to be giant (also Shenoy et al. object); our type } \\
\text { is M8 }\end{array}$ \\
\hline $043248.0+223952$ & GSC 01829-01009 = 2MASS J04324806+2239523 & Bright, assumed to be giant; our type is M9 \\
\hline $043453.4+270534$ & 2MASS J04345345+2705346 & Bright, assumed to be giant; our type is $\mathrm{K} 7$ with substantial $A_{V}$ \\
\hline $043706.6+214241$ & $\ldots$ & Bright, assumed to be giant; our type is $\mathrm{K} 2-3$ \\
\hline $043856.3+271642$ & HD 283753 & Bright, assumed to be giant; literature type is F6III \\
\hline $044118.4+240157$ & $\ldots$ & $\begin{array}{l}\text { Bright, assumed to be giant (also Shenoy et al. object); our type } \\
\text { is M5 }\end{array}$ \\
\hline $044401.6+252014$ & DO 10700 & Bright, assumed to be giant; our type is M5 \\
\hline $044435.0+250108$ & $\ldots$ & $\begin{array}{l}\text { Bright, assumed to be giant (also Shenoy et al. object); our type } \\
\text { is M9 }\end{array}$ \\
\hline $044510.7+244156$ & IRC +20091 & Bright, assumed to be giant; our type is M8 \\
\hline $044659.2+253657$ & IRC $+30094=2$ MASS J04465929+2536575 & Bright, assumed to be giant \\
\hline \multicolumn{3}{|c|}{ Insignificant IR excess } \\
\hline $041501.1+250432$ & $\ldots$ & Our type is G8-K0 \\
\hline $041539.2+235344$ & $\ldots$ & (Unobserved) \\
\hline $041643.3+244717$ & NLTT 12897 & (Unobserved) \\
\hline $041702.9+242425$ & $\ldots$ & Our type is $\mathrm{K} 0-\mathrm{K} 2$ \\
\hline $041707.0+293104$ & $\ldots$ & Our type is K3 \\
\hline $041734.0+240153$ & $\ldots$ & Our type is G6 \\
\hline $041934.0+252302$ & $\cdots$ & Our type is $\mathrm{K} 2$ \\
\hline $042216.3+262635$ & $\ldots$ & Our type is G3-G5 \\
\hline $042359.7+251452$ & $\ldots$ & Our type is M4 \\
\hline $042414.2+252350$ & $\ldots$ & Our type is F5 \\
\hline $042635.1+254223$ & $\ldots$ & Our type is G6 \\
\hline $042739.6+254208$ & $\ldots$ & Our type is F0 \\
\hline $042834.4+262104$ & $\cdots$ & (Unobserved) \\
\hline $042859.4+273625$ & $\cdots$ & Our type is $\mathrm{B} 9-\mathrm{A} 0 \mathrm{w} / \mathrm{substantial} A_{V}$ \\
\hline $043040.8+235034$ & $\cdots$ & Our type is F6 \\
\hline $043203.7+241223$ & $\ldots$ & Our type is F0 \\
\hline
\end{tabular}


Table 11

(Continued)

\begin{tabular}{|c|c|c|}
\hline SST Tau Number & Other Names & Notes \\
\hline $043223.4+230059$ & $\ldots$ & Our type is $\mathrm{K} 2-\mathrm{K} 3 \mathrm{w} /$ substantial $A_{V}$ \\
\hline $043237.5+292556$ & $\ldots$ & Our type is F0 \\
\hline $043254.0+294538$ & $\ldots$ & Our type is G6-K0 \\
\hline $043304.9+253705$ & $\ldots$ & Our type is $\mathrm{K} 0-\mathrm{K} 2$ \\
\hline $043335.6+242800$ & 2MASS J04333567+2428004 & Our type is $\mathrm{K} 2 \mathrm{w} /$ substantial $A_{V}$ \\
\hline $043348.2+274400$ & $\ldots$ & Our type is F0 \\
\hline $043354.3+285413$ & $\cdots$ & (Unobserved) \\
\hline $043433.3+244312$ & IRAS $04315+2436$ & Our type is M5 \\
\hline $043458.8+240958$ & 2MASS J04345881+2409587 & Our type is $\mathrm{K} 2$ \\
\hline $043544.2+215743$ & $\ldots$ & Our type is F0 \\
\hline $043822.9+221048$ & $\ldots$ & Our type is $\mathrm{K} 2-\mathrm{K} 3$ \\
\hline $043853.4+251909$ & $\ldots$ & Our type is F8 \\
\hline $044018.8+243234$ & $\cdots$ & Our type is G5 \\
\hline $044146.7+253824$ & [THL2004] 2MASS TMRS23 & Reported as potential Taurus member in the literature \\
\hline $044216.7+263917$ & $\ldots$ & Our type is $\mathrm{K} 2$ \\
\hline $044329.3+260818$ & $\ldots$ & Our type is $\mathrm{K} 1$ \\
\hline $044334.9+263505$ & $\ldots$ & Our type is G8-K2 \\
\hline $044348.0+242723$ & $\ldots$ & Our type is $\mathrm{K} 0-\mathrm{K} 2$ \\
\hline $044415.9+255300$ & $\cdots$ & Our type is F1 \\
\hline $044453.1+261257$ & $\ldots$ & Our type is A9-F0 \\
\hline $044700.1+242745$ & $\ldots$ & Our type is G8-K2 \\
\hline $044734.9+234528$ & $\ldots$ & Our type is M2 \\
\hline $044827.1+263006$ & $\ldots$ & Our type is F0-F1 \\
\hline $044931.5+244935$ & $\ldots$ & Our type is $\mathrm{K} 5$ \\
\hline \multicolumn{3}{|c|}{$8 \mu \mathrm{m}$ pop-ups } \\
\hline $041136.6+293522$ & $\ldots$ & Likely galaxy \\
\hline $041351.6+263819$ & 2MASX $04135166+2638195$ & Likely galaxy \\
\hline $041447.7+254956$ & 2MASX $04144776+2549564$ & Likely galaxy \\
\hline $041525.4+245339$ & 2MASX $04152538+2453388$ & Likely galaxy \\
\hline $041529.1+245942$ & $\ldots$ & Likely galaxy \\
\hline $041556.9+261559$ & $\ldots$ & Likely galaxy \\
\hline $041613.6+253134$ & 2MASX 04161364+2531351 & Likely galaxy \\
\hline $041711.4+282157$ & $\ldots$ & Likely galaxy \\
\hline $041718.0+291940$ & $\ldots$ & Likely galaxy \\
\hline $041742.5+275427$ & $\ldots$ & Likely galaxy \\
\hline $041756.2+250622$ & $\ldots$ & Likely galaxy \\
\hline $041829.1+285127$ & $\cdots$ & Likely galaxy \\
\hline $041842.1+283543$ & $\ldots$ & Likely galaxy \\
\hline $041843.7+250715$ & $\ldots$ & Likely galaxy \\
\hline $041903.8+271552$ & $\ldots$ & Likely galaxy \\
\hline $041916.1+250413$ & $\ldots$ & Likely galaxy \\
\hline $041929.7+253802$ & $\ldots$ & Likely galaxy \\
\hline $041950.1+251009$ & $\ldots$ & Likely galaxy \\
\hline $042043.7+252715$ & $\ldots$ & Likely galaxy \\
\hline $042047.0+282000$ & $\ldots$ & Likely galaxy \\
\hline $042140.8+282611$ & 2MASX $04214085+2826119$ & Likely galaxy; spectroscopically confirmed xgal \\
\hline $042159.7+234810$ & $\ldots$ & Likely galaxy \\
\hline $042252.9+225507$ & 2MASX $04225293+2255071$ & Likely galaxy; spectroscopically confirmed xgal \\
\hline $042257.6+243206$ & $\ldots$ & Likely galaxy \\
\hline $042342.1+235903$ & $\ldots$ & Likely galaxy \\
\hline $042343.7+245945$ & $\ldots$ & Likely galaxy \\
\hline $042417.0+234144$ & $\ldots$ & Likely galaxy \\
\hline $042417.9+272942$ & $\ldots$ & Likely galaxy \\
\hline $042429.2+224613$ & $\ldots$ & Likely galaxy \\
\hline $042521.7+262249$ & 2MASX $04252174+2622493$ & Likely galaxy \\
\hline $042634.9+260816$ & 2MASS J04263497+2608161 & Likely galaxy; spectroscopically confirmed xgal \\
\hline $042654.0+262921$ & $\ldots$ & Likely galaxy \\
\hline $042843.2+274001$ & $\ldots$ & Likely galaxy \\
\hline $043028.2+280420$ & $\ldots$ & Likely galaxy \\
\hline $043044.5+282640$ & $\cdots$ & Likely galaxy \\
\hline $043114.1+245838$ & $\cdots$ & Likely galaxy \\
\hline $043205.6+220627$ & $\ldots$ & Likely galaxy \\
\hline $043241.0+251308$ & $\ldots$ & Likely galaxy \\
\hline $043303.9+280846$ & $\ldots$ & Likely galaxy \\
\hline $043329.4+262052$ & $\ldots$ & Likely galaxy \\
\hline
\end{tabular}


Table 11

(Continued)

\begin{tabular}{|c|c|c|}
\hline SST Tau Number & Other Names & Notes \\
\hline $043407.2+280154$ & $\ldots$ & $\begin{array}{l}\text { Likely galaxy; very noisy spectrum, could be consistent with low- } z \text { galaxy } \\
\text { or late G/early K }\end{array}$ \\
\hline $043446.1+290451$ & 2MASX $04344611+2904516$ & $\begin{array}{l}\text { Identified by both Gutermuth and c2d methods as likely YSO, but SED } \\
\text { suggests likely galaxy; spectroscopically confirmed xgal }\end{array}$ \\
\hline $043456.7+232501$ & 2MASX $04345670+2325016$ & Likely galaxy \\
\hline $043513.3+232449$ & $\ldots$ & Likely galaxy \\
\hline $043516.1+213943$ & $\ldots$ & Likely galaxy \\
\hline $043712.3+260733$ & $\ldots$ & Likely galaxy \\
\hline $043820.5+215748$ & 2MASX $04382057+2157489$ & Likely galaxy \\
\hline $043828.3+253223$ & $\ldots$ & Likely galaxy \\
\hline $043931.8+214742$ & $\ldots$ & Likely galaxy \\
\hline $043958.0+253354$ & $\ldots$ & Likely galaxy \\
\hline $044114.8+253242$ & $\ldots$ & Likely galaxy \\
\hline $044308.1+240957$ & $\ldots$ & Likely galaxy \\
\hline $044554.8+240843$ & IRAS04428+2403 & Galaxy; listed by Kenyon et al. (2008) as confirmed Taurus member \\
\hline $044614.5+253100$ & $\ldots$ & Likely galaxy \\
\hline $044717.7+234529$ & 2MASX $04471766+2345296$ & Likely galaxy \\
\hline $044933.6+234518$ & $\ldots$ & Likely galaxy \\
\hline
\end{tabular}

Note. ${ }^{\text {a }}$ These objects were selected at various points in our selection process, but we have rejected these as YSOs. See associated text for much more information.

Table 12

Literature Non-members Regarded as Potential New Members ${ }^{\mathrm{a}}$

\begin{tabular}{|c|c|c|}
\hline SST Tau Number & Other Names & Notes \\
\hline $041803.3+244009$ & 2MASS $04180338+2440096$ & Needs additional follow-up; our type is A9 \\
\hline $041810.7+251957$ & [GBM90] L1506 1 & Probable new member, our type is K8-M0 \\
\hline $041823.2+251928$ & 2MASS $04182321+2519280$ & Needs additional follow-up; our type is G: \\
\hline $042212.9+254659$ & 2MASS $04221295+2546598$ & Pending follow-up \\
\hline $042518.6+255535$ & 2MASS $04251866+2555359$ & Shenoy et al. object; needs additional follow-up; our type is M5 \\
\hline $042920.8+274207$ & 2MASS $04292083+2742074$ & Possible new member; our type is M6. \\
\hline $043024.1+281916$ & 2MASS 04302414+2819165 & Shenoy et al. object; needs additional follow-up; our type is M5 \\
\hline $043042.8+274329$ & 2MASS 04304284+2743299 & Shenoy et al. object; needs additional follow-up; our type is M6 \\
\hline $043228.1+271122$ & 2MASS $04322815+2711228$ & Shenoy et al. object; needs additional follow-up; our type is M6 \\
\hline $043344.6+261500$ & 2MASS $04334465+2615005$ & New member; Shenoy et al. object; our type is M6e \\
\hline $043435.4+264406$ & 2MASS 04343549+2644062 & Shenoy et al. object; needs additional follow-up; our type is M3 \\
\hline $044125.7+254349$ & 2MASS $04412575+2543492$ & Pending follow-up \\
\hline $044539.8+251704$ & 2MASS $04453986+2517045$ & Shenoy et al. object; needs additional follow-up; our type is M5 \\
\hline $044557.0+244042$ & 2MASS $04455704+2440423$ & Needs additional follow-up; our type is $\mathrm{K} 2$ \\
\hline $044639.8+242526$ & 2MASS $04463986+2425260$ & Shenoy et al. object; needs additional follow-up; our type is M5 \\
\hline
\end{tabular}

Note. ${ }^{\text {a }}$ These objects were noted in the literature as likely non-members, but we have promoted them to be candidate members based on our Spitzer observations. See text for more information.
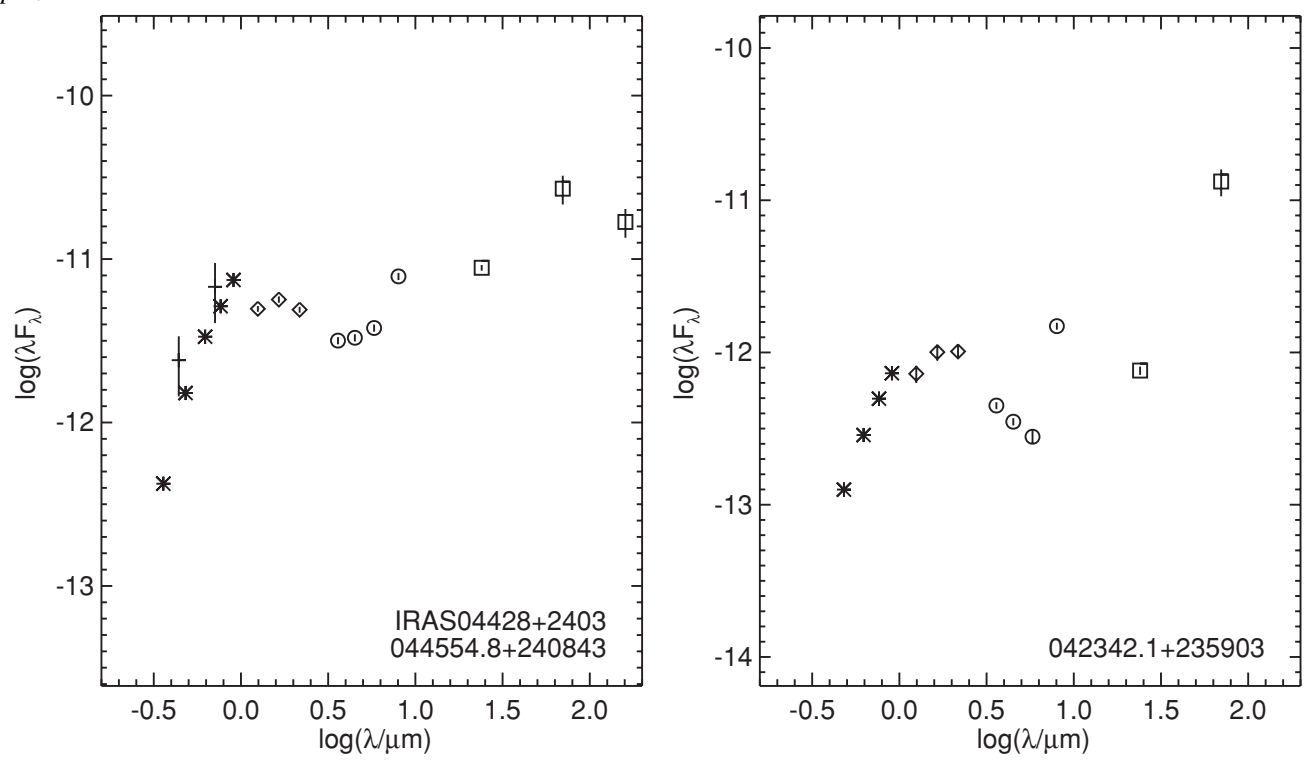

Figure 15. Example $8 \mu \mathrm{m}$ pop-up SEDs for two objects, $044554.8+240843=$ IRAS $04428+2403$ and $042342.1+235903$. Notation is the same as for all of the other SEDs in this paper. Both of these objects are likely to be galaxies. 
Taurus members, and we have excluded them from the list of candidates.

\section{APPENDIX C}

\section{LITERATURE NON-MEMBERS REGARDED AS POTENTIAL NEW MEMBERS}

Several objects listed in the literature as non-members (e.g., assumed but not spectroscopically confirmed background objects) appear as having colors consistent with YSOs. In several cases, the literature regarded them as non-members not because of spectroscopic confirmation, but because of optical properties. Since we investigated their IR properties for the first time, and found them to have IR excesses, we have at least for the time being promoted them back to being YSO candidates rather than non-member candidates. These objects are all identified in the relevant tables above (including Table 12), and since they are still YSO candidates, their SEDs appear in Appendix A.

Shenoy et al. (2008) use the data from our Taurus-1 survey (as reported by Luhman et al. 2006) combined with 2MASS to create a catalog of objects they believe to be background objects. Several of the objects they report as candidate background objects are based on 2MASS-only measurements (no IRAC). Now, with the addition of the MIPS-24 data (as well as the IRAC data for the rest of the survey), many of them show up as likely YSO candidates in at least one of our color spaces we investigated. We have promoted several of the objects reported in Shenoy et al. (2008) to potential members; they are listed and identified in the relevant tables in the main body of the paper, but listed in Table 11 as just "Shenoy et al. object." Most of these objects do not have obvious signs of youth in the spectra we have, so additional data are needed to determine if they are members.

A few objects merit special discussion. The first, $041810.7+251957$ ([GBM90] L1506 1), is listed by Goodman et al. (1990) as one of the objects for which the authors measured polarimetry, and therefore they assumed was a background object, but is not called out as anything remarkable in any other sense. It appears as a strong YSO candidate in all of the colormagnitude spaces we investigated. We have it listed in the tables above as a new member, with a spectral type that we determined to be K8-M0, substantial emission lines in the spectrum, and a YSO SED class of II.

The second object of note is $042920.8+274207$ (2MASS $04292083+2742074)$, which appears in Luhman et al. (2009b) as a background giant, M5III. We report it as a possible new member based on all the information we have; our type is M6 with low gravity. It shares many characteristics of young stars, but also those of some post-main-sequence objects. Further investigation is needed.

\section{REFERENCES}

Alcalá, J., et al. 1996, A\&AS, 119, 7

Alksnis, A., Balklavs, A., Dzervitis, U., Eglitis, I., Paupers, O., \& Pundure, I. 2001, Balt. Astron., 10, 1

Allen, L. E., et al. 2004, ApJS, 154, 363

Audard, M., et al. 2007, A\&A, 468, 379

Aumann, H., et al. 1984, ApJ, 278, 23

Barrado y Navascués, D., \& Martín, E. 2003, AJ, 126, 2997

Bary, J., et al. 2007, BAAS, 21115406

Beichman, C., et al. 1986, ApJ, 307, 337

Bessell, M. S. 1979, PASP, 91, 589
Carpenter, J., et al. 2001, AJ, 121, 3160

Chamaraux, P., Kazes, I., Saito, M., Yamada, T., \& Takata, T. 1995, A\&A, 299, 347

Chen, B., et al. 2004, "OM Calibration Status," XMM-Newton OM calibration documentation http://xmm.vilspa.esa.es/sas/7.0.0/watchout/Evergreen_tips_ and_tricks/uvflux.shtml

Cieza, L., \& Baliber, N. 2007, ApJ, 671, 605

Cohen, M., et al. 1989, ApJ, 339, 455

Cox, A. N. (ed.) 2000, in Allen's Astrophysical Quantities (4th ed.; Berlin: Springer)

D’Alessio, P., et al. 1999, ApJ, 527, 893

Edwards, S., et al. 1993, AJ, 106, 372

Elias, J. 1978, ApJ, 224, 857

Evans, N. J., et al. 2003, PASP, 115, 965

Evans, N. J., et al. 2007, c2d delivery document, SSC Web site

Evans, N. J., et al. 2009, ApJS, 181, 321

Fazio, G., et al. 2004, ApJS, 154, 10

Finkbeiner, D., et al. 2004, AJ, 128, 2577

Flaccomio, E., et al. 2003, A\&A, 402, 277

Gautier, T. N., et al. 2007, ApJ, 667, 527

Geers, V., et al. 2009, A\&A, 495, 837

Goldsmith, P., et al. 2008, ApJ, 680, 428

Goodman, A., et al. 1990, ApJ, 359, 363

Grankin, K., et al. 2008, A\&A, 479, 827

Güdel, M., Padgett, D., \& Dougados, C. 2007a, in Protostars and Planets V, ed. B. Reipurth, D. Jewitt, \& K. Keil (Tucson, AZ: Univ. of Arizona Press), 329

Güdel, M., et al. 2007b, A\&A, 468, 353

Guieu, S. 2008, PhD thesis, Univ. of Grenoble

Guieu, S., et al. 2006, A\&A, 446, 485

Guieu, S., et al. 2007, A\&A, 465, 855

Gutermuth, R., et al. 2008, ApJ, 674, 336

Gutermuth, R., et al. 2009, ApJS, 184, 18

Hartmann, L., et al. 2005, ApJ, 629, 881

Harvey, P., et al. 2007, ApJ, 663, 1149

Hawarden, T., et al. 2001, MNRAS, 325, 563

Herbig, G. H., \& Kameswara Rao, N. 1972, ApJ, 174, 401

Hernandez, J., et al. 2008, ApJ, 686, 1195

Jørgensen, J., et al. 2006, ApJ, 645, 1246

Kenyon, S., et al. 1994, AJ, 108, 251

Kenyon, S., et al. 2008, in the Handbook of Star Forming Regions I, ed. B. Reipurth (The Northern Sky ASP Monograph Publications, Vol. 4), 405 Lonsdale, C., et al. 2003, PASP, 115, 897

Lowrance, P., et al. 2003, ApJ, 584, L95

Luhman, K., et al. 2006, ApJ, 647, 1180

Luhman, K., et al. 2009a, ApJ, 691, L1265

Luhman, K., et al. 2009b, ApJ, 703, 399

Makovoz, D., \& Marleau, F. 2005, PASP, 117, 1113

Malfait, K., et al. 1998, A\&A, 332, L25

Monin, J.-L., et al. 2007, BAAS, 211, 2906

Monin, J.-L., et al. 2010, A\&A, submitted

Motte, F., \& André, P. 2001, A\&A, 365, 440

Ogura, K., Sugitani, K., \& Pickles, A. 2002, AJ, 123, 2597

Oliveira, I., et al. 2009, ApJ, 691, 672

Padgett, D., et al. 2008a, Taurus Legacy Delivery Document (P08), available from http://ssc.spitzer.caltech.edu/legacy/taurushistory.html

Padgett, D., et al. 2008b, ApJ, 672, 1013

Padmanabhan, N., et al. 2008, ApJ, 674, 1217

Rebull, L. 2001, AJ, 121, 1676

Rebull, L., et al. 2000, AJ, 119, 3026

Rebull, L., et al. 2006, AJ, 131, 2934

Rebull, L., et al. 2007, ApJS, 171, 447

Rieke, G., et al. 2004, ApJS, 154, 25

Scelsi, L., et al. 2007, A\&A, 468, 405

Scelsi, L., et al. 2008, A\&A, 490, 601

Shenoy, S., et al. 2008, ApJS, 176, 457

Siess, L., Dufour, E., \& Forestini, M. 2000, A\&A, 358, 593

Skrutskie, M., et al. 2006, AJ, 131, 1163

Slesnick, C. L., Hillenbrand, L. A., \& Carpenter, J. M. 2008, ApJ, 688, 377

Tobin, J., et al. 2008, ApJ, 679, 1364

Torres, R., et al. 2007, ApJ, 671, 1813

Torres, R., et al. 2009, ApJ, 698, 242

Weedman, D., \& Houck, J. 2009, ApJ, 693, 370

Werner, M., et al. 2004, ApJS, 154, 1

Wilking, B., et al. 2001, ApJ, 551, 357

Wolk, S., et al. 2006, AJ, 132, 1100 